qQL

444

C7B7 Z

INVZ 

Cardid 1949

\author{
WILSON COPEPOD LIBRARY \\ Smithsonian Institution \\ Invertebrate Zoology \\ (Crustacea)
}







\title{
ALESSANDRO BRIAN
}

\author{
Dottore in Scienze Naturali
}

WILSON COPEPOD LIBRARY

Smithsonian Institution

Invertebrate Zoology

(Crustacea)

\section{COPEPOII PARASSITI}

\section{Pesci d'Italia}

(Con XXI Tavole)

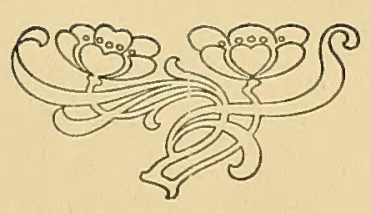

\section{GENOVA}

STAB. TIPO-LITOGRAFICO R. ISTITUTO SORDOMUTI 

dove ebbi un tavolo di studio per gentile concessione del Ministro dell'istruzione pubblica e del Direttore e Fondatore di detto celebre Istituto, Prof. Dohrn, ai quali esprimo la mia gratitudine. Colà ho potuto eseguire da materiale fresco e quasi ancor vivente, una parte dei disegni a colori che corredano questo libro.

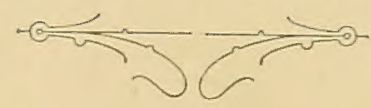

Cooperarono alla raccolta del materiale di copepodi parassiti dei pesci, da me studiato, che si conserva nel Museo Zoologico della R. Università di Genova, oltrechè il prof. C. Parona i signori professori F. Mazza, E Setti, V. Ariola, G. Damiani, il compianto A. Perugia e sopratutto il preparatore sig. B. Borgioli, ai quali tutti rivolgo i miei ringraziamenti. 


\section{STOR I A.}

\section{Copepodi lerneiformi.}

La prima rudimentale conoscenza dei copepodi parassiti dobbiamo ricercarla in tempi molto antichi.

Aristotile (384-32r av. C.), la mente più vasta e più nudrita di studi, l'osservatore più diligente e profondo di tutta l'antichità, è stato senza dubbio il primo a darci notizie sull' esistenza di questi animali. Nella sua "Historia Animalium, ricorda che il tonno e il pesce spada sono tormentati da una specie di verme, che si attacca da sè stesso sotto la natatoria e causa tale irritazione all'animale che esso sovente salta fuori dell' acqua e cade sul bordo dei bastimenti. "Thunni et gladii agitantur asilo canis exortu, habent enim utrique per id tempus sub pinna ceu vermiculum, quem asilum vocant, effigie scorpionis, magnitudine aranei; infestat hoc tanto dolore, ut non minus interdum gladius quam delphinus exiliat, unde fit, ut vel in navigia saepenumero incidat. » Lib. VIII, Cap. I9.

Plinio il vecchio, cultore di lettere e scienze, nella sua "Historia Naturalis, , piì che trattato di storia naturale, enciclopedia del sapere di quei tempi, non mancò di ripetere 1'asserzione di Aristotile quasi colle parole stesse di lui. "Animal est parvum, scorpionis effigie, aranei magnitudine. Hoc se, et thynno, et ei qui gladius vocatur, crebro delphini magnitudine excedenti, sub pinna affigit aculeo, tantique infestat dolore, ut in naves saepenumero exiliant. Quod et alias faciunt aliorum vim timentes, mugiles maxime, tam praecipuae velocitatis, ut transversa navigia interim superjactent. » Lib. IX, cap. I6.

Oppiano di Cicilia, vissuto in tempi che correvano poco favorevoli per la poesia e per la letteratura ellenica, proclivi quali erano alla erudizione e ad una cultura varia e curiosa, compose, verso il 180 dopo Cristo, un poemetto didascalico mitologico sulla pesca (Alieutica in 5 libri) povero d'ispirazione, in cui descrisse le sofferenze del povero tonno e del pesce spada in linguaggio patetico, e asseriva che i pesci sono spesso uccisi dai loro assalitori pigmei (').

(1) Al Tonno, e al Pesce spada ougnor va dietro Danno compagno, che non pônno mai Lassare, o pur fuggire, nelle pinne Sedente, crudo Assillo, che nel tempo Che spunta il cane torrido, ed arsiccio, Del mortal ago la veloce appoggia Forza, ficcato assai acutamente,

E fiera rabbia desta, di dolori Armando, e instiga contro voglia al ballo Con ispesso flagello: e quei da negra Puntura enfiati infuriano; ed or quinci,
Or quindi si cavalcan per lo flutto Infinito, tenendo immenso affanno, Sovente s'imbattcr nelle cornute Ben antennate navi, a corsa spinti Distemperata, c sovente dal falso Flutto sbalzaro, e scorsero per terra Palpitando, e i dolori assai gagliardi Barattaro alla morte; tal gravoso Morso l'ingombra, e giù caccia nel fondo; Nè li abbandona o refrigerio lascia. 
Ateneo grammatico greco, nativo di Naucrate, fiorito dopo la morte d'Augusto e vissuto lungo tempo a Roma, celebre per la sua opera "Deipnosophistae " (Banchetto degli eruditi), in I 5 libri, stata tradotta più volte, preziosa per la storia delle lettere, delle scienze, dei costumi e dei mestieri, contenendo quasi isoo estratti di scrittori perduti, ripete ciò che i suoi predecessori avevano scritto a riguardo di questi parassiti.

Queste sono le poche notizie relative al nostro argomento, che si possono trarre dalle opere dei Greci e dei Romani, notizie che danno un concetto delle scarse e grossolane cognizioni loro a riguardo di tali parassiti. Ed esse, a dire il vero, sono dovute ad osservazioni originali molto imperfette da parte di un solo, cioè di Aristotile, a cui le varie scienze mettono capo e la zoologia prima di tutte. Da lui comincia la storia dei nostri crostacei.

Sebbene sia evidente che egli col termine oibros: (oestrus sive asilus marinus) abbia voluto accennare ad un copepode lerneino, non tutti gli odierni naturalisti vanno d'accordo sulla sua interpretazione.

Gerstaecker nel classico trattato ( $\left.{ }^{1}\right)$ ritiene che si possa riferire tanto ad un caligide (Cecrops) quanto ad un isopodo parassita, ciò nondimeno è difficile, egli scrive, di stabilire il vero, per mancanza di cognizioni sicure a questo proposito.

Boccone di già nel XVII secolo pensava che si trattasse di una Pennella. Per primi Steenstrup e Lütken nel i $865\left({ }^{2}\right)$ dimostrarono che l'animale in questione è un lerneopodide, ossia precisamente la Brachiella Thynni di Cuvier, ciò che secondo me è più che certo. Difatti questo copepode parassita si trova con una certa frequenza sul tonno e sempre sotto l'angolo delle pinne: è di grandezza abbastanza notevole, e nelle sue forme esterne foggiato così stranamente da eccitare la fantasia dei primi osservatori. Nulla di più naturale che Aristotile abbia creduto di intravedere in questo animaluccio la forma dello scorpione, ritenendolo parassita molto doloroso all'ospite, a causa delle varie appendici da cui è costituito, somiglianti a piedi ma probabilmente scambiate per organi succhianti. Le quattro appendici caudali sottili ed allungate, i due sacchi oviferi parimente protesi all'indietro colle due braccia esili e il lungo collo pure assottigliato e sporgente in avanti, danno certamente all' animale un aspetto che doveva imbarazzare assai nella descrizione anche l'osservatore di quei tempi di maggiore ingegno. Non deve perciò meravigliarci se Aristotile paragonandolo per grandezza al ragno, non abbia saputo trovare altro insetto che 10 scorpione per confrontarlo nell'aspetto esterno. D'altro lato non dimentichiamo che egli riconobbe in questo lerneopodide la natura di verme giacchè lo chiamò vermiculum.

Caduto 1'impero romano e tramontati gli ultimi avanzi della rifioritura ellenica nelle lettere e scienze dopo Alessandro, coll' età bizantina e col medio evo cominciò per tutte le scienze e specie per la zoologia quel lunghissimo periodo di tempo, che Victor Carus chiamò, con felicissima espressione, - periodo del silenzio durato per le scienze tutte fino al XII secolo e più lungamente in ispecial modo

(1) V. p. 592 dell' op. cit. nella Bibliografia.

$\left({ }^{2}\right)$ V. p. $f^{2}$ I dell'op. cit. nella Bibliografia. 
per la nostra carcinologia, per quanto compensato, a dire il vero, dal luminoso progresso dovuto alla scuola araba. Quest'ultima epoca non ha dato indizio, per quanto io sappia, di conoscere l'esistenza dei copepodi parassiti.

Pur troppo il silenzio continuò profondo per secoli e cioè fino dopo il XII; e se nei secoli XIII, XIV e XV, la zoologia cominciò a svilupparsi, ciò fu soltanto per quello che riguarda gli animali vertebrati principalmente; ma ben poco per gli altri e nulla pei crostacei. È d'uopo trascorrere tutto il medio evo e non breve tempo susseguente per trovare traccia di nuove notizie su di questi animali e giungere ai secoli XVI e XVII, allorquando col rifiorire delle scienze, anche la zoologia fu maggiormente coltivata. Tuttavia i primi naturalisti di questo nuovo periodo non ci fecero conoscere nulla di nuovo, essi si riferirono agli scritti di Aristotile e di Plinio.

Ippolito Salviani, italiano, nativo di Città di Castello (I5I4-I572) nella sua «Aquatilium Animalium Historia » (con 99 tavole in rame) pp. I 26-8, cita a lungo i passaggi riferentisi all'argomento datoci da Aristotile, Plinio, Oppiano e Ateneo.

Parimente Guglielmo Rondelet, naturalista nato a Montpellier (I507I566), nei suoi « Libri de Piscibus Marinis, » ripete gli accenni dei primi autori sul parassita del tonno e del pesce spada, e a provare la sua personale conoscenza del piccolo animale in discorso, ci dà una figura di un tonno, col parassita attaccato presso della pinna pettorale. Esso aderisce, scrive egli, così tenacemente, che non può essere rimosso da nessuna scossa, dal corpo del suo ospite $\gg\left({ }^{~}\right)$.

Corrado Gessner celebre naturalista, detto il Plinio tedesco, nativo di Zurigo (I516-1565) nella sua "Historia A nimalium - De Aquatilibus », I558, entra largamente a discorrere di questo stesso animale. Egli descrive la sua struttura ed aspetto, e dice: «Poca gente sa che questo parassita, per essere molto piccolo, si vede raramente, fuorchè nel tempo che si leva la canicola e allora si nota non su parecchi pesci, ma solamente sul tonno, pesce spada, e occasionalmente sul delfino (e non su ciascuno individuo). » Egli dà una figura di esso poco ingrandita (p. I 2, fig. annessa), e ripete il disegno del parassita in situ sul tonno (p. I 152, fig. an.), come precedentemente l' aveva data Rondelet. "Detto animale aderisce cosi solidamente, egli osserva, che non può essere rimosso senza essere strappato. Succhia il sangue del pesce, come fa la sanguisuga, fino a che cade per troppa pienezza, e allora muore. » Dopo questa considerazione egli rileva che questi pesci, (il tonno specialmente è menzionato) sono magri e cattivi durante il forte dell'estate, dovuto ciò all' essere così miserabilmente tormentati da tale contagio, e sono più facilmente presi in questo tempo che in inverno, nella quale stagione sono in migliore condizione (pp. I I 2, II 3). L'esemplare che egli descrive, avendolo esaminato egli stesso, era di un colore bianco, e fu trovato aderente « ad piscem Pagrum».

(1) Vedasi qui riprolotto a tav. XIX, fig. 6 del presente mio lavoro, il cattivo disegno dell'Oestrus sive Asilus di Rondelet come is stato eseguito da questo autore, e pubblicato a p. IIz della sua opera "De Piscibus Marinis," in un'antica edizionc del 1554, stampata a Lione, apud Mathhian Bouthomme. 
Finora oggetto di attenzione da parte di questi naturalisti ora ricordati è sempre la Brachiella del tonno. Il primo ad occuparsi di una specie diversa è stato Tommaso Moufet, il quale nel r634, nel suo «Theatrum Insectorum», Cap. 38, p. $32 x$, dà una figura molto rozza di animale, che accanto ad un isopodo parassita, distingue come Pediculus marinus, e che sembra al Gerstaecker doversi riferire ad un pidocchio di pesce, forse al gen. Dichelesthium?

Francesco Redi (n. Arezzo, I8 febbr. I626; m. Pisa I marzo I694) naturalista, medico e letterato insigne che colla sua opera lasciò orme incancellabili nella storia naturale e nella elmintologia, non conobbe, a dire il vero, alcuno dei copepodi parassiti dei pesci. A lui per contro spetta il merito incontestato di avere scoperto il primo copepode di forma regolare, avendo fatto conoscere il Doropygus della Mentula (Osserv. intorno agli animali viventi che si trovano negli animali viventi, Firenze 1684), mentre si attribuiva questo merito a Stephan Blankaart per una cattiva figura di Cyclops? pubblicata in Amsterdam nel I868.

Don Antonio Giuseppe Pernetty scrittore francese, nato a Roanne nel I7 16, morto a Valence nel I80 I, facendo parte come elemosiniere della spedizione di Bougainville alle isole Malouines ( $1763-4)$, ne pubblicò la relazione a Parigi nel 1770, e in questa sua opera (') fa conoscere, senza saperlo, la specie stessa che aveva attirato l'attenzione di Aristotile e suoi imitatori (T. I, p. 93). La trovò aderente ad un tonno nell'Atlantico e ne diede due figure nella Pl. I, fig. 5 e 6, che mi sembrano rappresentare con verità la Brachiella Thynni.

Più tardi Enrico Maria Ducrotay de Blainville, celebre zoologo e anatomista, nativo di Arques ( $1778-1850$ ), illustra questa stessa forma di copepode. chiamandola Lerneomyzon incisa (Journal de Physique, xcv, 1822) e presentando una figura nella quale Baird (1850) riconosce il medesimo animale descritto da Pernetty.

Boccone, un naturalista siciliano, nelle sue « Ricerche e Osservazioni Naturali » pubblicate in Amsterdam, nel 1674, ci informa che a Messina la sua attenzione fu richiamata dal Sig. Scilla, un celebre pittore e antiquario di quella città, dal fatto che lo Xiphias, o pesce spada, era bene conosciuto dai pescatori della costa per essere tormentato da un parassita che essi chiamano sanguisuga. La sola informazione che egli ricevette si era che l'animale agiva similmente ad un succhiello che penetrava da sè stesso nella carne del pesce. Gli avvenne tuttavia di ottenere un esemplare, che egli descrive e figura (op. cit. p. 284 e seguenti, tav. p. 287). Esso seppellisce, egli scrive, la sua intera testa o tronco nella carne del pesce spada. E tale parassita può fissarsi su qualunque parte del corpo dell'ospite, ma non si mette maỉ vicino alle pinne perchè queste, coi loro movimenti, non arrivino a danneggiarlo.

Boccone erroneamente riferisce questa specie all' oั̃ di Gessner ecc. e aggiunge che non è stata data la figura di esso nè da Gessner nè da altri. Evidentemente questo naturalista ha solo dato importanza al fatto che il parassita si trova sullo Xiphius o pesce spada e quindi si è ingannato ritenendolo per la forma nota da Aristotile, che questi indicava a torto non solo sui tonni ma

(1) Pernetty A. G. - Hisloire d'un voyage aux lles Maloumes, Paris, 1770. 
anche sui pesci spada; ma se avesse osservato le figure date da Gessner e da Rondelet, egli non avrebbe commesso l'errore di asserire le due specie essere identiche. La forma descritta da Boccone è stata riconosciuta da Baird (r850) per una Pennella ( $P$. fllosa parassita clello Xiphias gladius).

Il naturalista siciliano a proposito di questo parassita ha fatto una curiosa osservazione: «questa sanguisuga, cosi scrive, sembra essere tormentata da una pulce che io non ho giammai visto su alcun altro animale. Essa è della grandezza di un pisello e sta attaccata da sè stessa solidamente sull' animale » p. 292. Gessner stesso nel citare la descrizione di Aristotile dell' asilus, aveva già esclamato " adeo nihil est quod hoste careat » p. Ir 2, e l'osservazione fatta da Boccone si spiega chiaramente colla presenza, notata da parecchi autori, di parassiti viventi sopra la Pennella. Voglio alludere al Conchoderma e alle Campanulariae cirripede il primo e idroidi i secondi, che si trovano a vivere come epocumeni o commensali su di detto copepode; associazione questa strana di una forma parassitaria sopra di un'altra parassita, che ebbi occasione anch'io di constatare a Genova su esemplari di Pennella.

Muraltus, nelle «Miscellanea curiosa sive Ephemeridum Medico Physicarum Germanicarum Academiae Naturae Curiosorum » pubblicate a Norimberga, nel i682, ricorda che mentre sezionava un esemplare di Mustela fuviatilis (Lota vulgaris Cuv.), egli trovò un insetto infisso nell'occhio e pendente fuori da questo. Esso era solidamente impiantato per mezzo delle braccia, e non vi era dubbio, scrive questo autore, che il parassita offendesse 1'occhio, si da rendere il pesce cieco.

Baker, nelle «Philosophical Transactions » 1744 , vol. XLIII, 35, f. 2, 3; descrive un «nuovo insetto marino da lui scoperto» alquanto simile al precedente che egli chiama «succhiatore d'occhio » e che trovò «fissato per mezzo del succhiatoio » all' occhio della sardina. La figura è eseguita molto malamente, tanto che, non è possibile determinarne la specie; ma noi sappiamo come una Lernaea infesti la comune sardina, ed è stata disegnata da I. Sow erby nelle «British Miscellany ». Si tratta probabilmente di questa specie, che oggi ha ricevuto il nome di Lernaeenicus sprattae Sow.

Tutti i predecessori di Linneo, che si occuparono di taluno dei crostacei, che qui trattiamo, sembrano aver fatte ben poche osservazioni personali di alcun rilievo. Anche quelli che diedero qualche notizia originale e nuova, non sapevano sceverarla da preconcetti ed errori grossolani, e la zoologia in quel tempo ne trasse ben poco profitto per la conoscenza di questo gruppo.

Solo da parte dei successori di Linneo, nei primi decenni del secolo XIX, come vedremo in seguito, si cominciò a raccogliere notizie di varie specie con osservazioni più esatte, e andò cosi preparandosi il terreno al periodo brillante per la storia dei nostri crostacei che comincia con Nordmann (1832). Ma prima ancora di arrivare a quest' epoca è interessante vedere quanto hanno fatto su questo argomento Linneo stesso e i suoi continuatori.

Nel ${ }_{74} 6$ Linneo, nella "Fauna Suecica " I." ediz., descrisse col nome di Lernaea cyprinacea un animale parassita trovato sopra il Cyprinus carassizus. Scbbene questa specie sia stata riconosciuta oggi come appartenente al genere Lernaeocera, tuttavia da essa è stato stabilito il genere Lernaea. Il celebre natu- 
ralista svedese nel suo "Iter Vestrog 》, 1747 , diede notizia di un'altra specie trovata sulle branchie di un Gadus, che egli chiamò Lernaea asellina ma alla quale oggi fu cambiato il nome in Oralien asellina. Nella sua seconda edizione della «Fauna Suecica», I76I, egli aggiunse una terza Lernaea, come abitante le branchie del salmone: e questa è la stessa che fu figurata e descritta da Gisler negli «Act. Holmens. (Acta Suecica): Kongl. Vetensk. Handling. », nel I 75 I, sotto il nome di Pediculus Salmonis, nome che.da Blainville fu sostituito con quello di Lernaeopoda salmonex.

Nel «Systema naturae», I 2 ediz., I 766, infine Lin neo aggiunse alla lista delle lernee una quarta specie, Lernaed branchialis, e queste quattro specie costituiscono tutto quello che il grande sistematico comprendeva nel genere Lernaea, che successivamente andò estendendosi in modo da riunire forme le più svariate e formare un grande gruppo o famiglia. $\mathrm{Ma}$ in seguito il genere ricordato fu ristretto entro limiti più angusti e naturali, e di varie specie si fecero generi nuovi (Lernaeocera, Lernaeenicus, Lernaeopoda, ecc.) Oggi non sono compresi col primitivo nome, che la forma tipica di Lemaea branchialis e solo tre o quattro altre specie.

Linneo nel descrivere tali esseri d'aspetto cosi bizzarro, non ebbe nessuna idea che appartenessero ai crostacei; al contrario classificò le forme sopra riferite, a causa della mollezza del loro corpo, nei molluschi, nella classe dei Vermes, secondo il suo sistema; e due altre forme di copepodi parassiti, che non abbiamo ancora citato, li comprese negli Zoophyti riferendoli al gen. Pennatula (Pennella).

I contemporanei di Linneo frattanto contribuirono non poco ad allargare la conoscenza sui copepodi lerneidi. Stroem (1762) descriveva la Lernaea adunca; Ellis (1763) la Pcnnatula filosa e P. sagitta; Baster (1765) la Lernaca Basteri (Blainv.); Goeze J. E. pubblicava nel I784 un lavoro interessante sulle lernee; Moder (1786) trattava di nuovo della Pennatula filosa e $P$. sagitta; A bildgaard (1794) illustrava la Lernaea anomala (Bramae); O. Fabricius (1794) faceva conoscere la Lernaea Lavareti; Holten (1802) la Lernaea Merlucci e L. Exocoeti; Hermann (1804) la Lernaca squamicola e L. Lotae; Delaroche(I8II) il Chondracanthus Zei e il Ch. Thynni; Major (1824) la Lemaeopoda stellata e L. salmonea; Grant (I827) la Lernaea elongata; e Retzius (1829) la Lemaea Dalmani.

Molte di queste forme sono conosciute oggi sotto altri nomi generici poichè in gran parte sono state tolte giustamente dal genere Lernnea.

I detti naturalisti tutti e anche altri editori e continuatori di Linneo, o piuttosto scrittori sistematici, hanno seguito le vedute del naturalista svedese nel ritenere che le lernee fossero vermi. Bruguière, nell” «Encyc Mèthod. », I792, e Blumenbach nel suo "Handbuch», I779, hanno adottato l'ordinamento stabilito da Linneo nel suo sistema. Cuvier, nel suo « Tableau Élementaire », 1798, poneva questi copepodi tra i Mollusca grasteropoda. L a m a r ck nel suo "Système des Anim. șans Vertèb. » I80r, ugualmente li comprende tra i molluschi, disponendoli coi nudi Mollusques cephalés. Bos c pure li ascrisse ai Mollusca; ma osserva che si avvicinano ai vermi intestinali. Lamarck, più tardi insoddisfatto di questa disposizione nella sua «Philosophie Zoologique, 》 I8og, li pone negli anellidi insieme alle planarie e alle sanguisughe. 
Però già in quel periodo di tempo contro l'opinione linneana di riguardare simili parassiti come vermi e ancora, per giunta, molluschi, cominciano a farsi sentire voci isolate, quà e là, già molto prima dell'insigne scoperta di Nordmann, in seguito alle investigazioni sullo sviluppo.

Lamarck stesso si avvide che la prima sua opinione non poteva essere esatta e più tardi, nel suo «Extrait du Cours de Zoologie, » I8I 2, mostra la necessità di formare una classe distinta per ricevere quelli, che egli chiama Epizocires, animali, che egli non può riferire esattamente ad alcuna delle classi già determinate del regno animale; e nella « Hist. Nat. Anim. sans Vertèb. » I. edit., i8I6, ponendo quelli tra gli Epizoaria, egli dice: «questi animali s'avvicinano ai vermi e agli insetti, senza appàrtenere nè agli uni nè agli altri. Essi indicano l'esistenza di una particolare serie, che probabilmente forma una classe nuova, e che può convenevolmente colmare la grande lacuna che esiste tra gli insetti e i vermi. »

Il celebre naturalista tedesco Lorenzo Oken (I779-I85I) professore a Jena, a Monaco ed a Zurigo, nel suo «Lehrbuch der Naturgeschichte, » I8 I5, seguendo Linneo nel porre le lernee tra i molluschi, fu però il primo ad iniziare la divisione di queste in differenti generi. Egli fino da quell' epoca aveva intraveduta la affinità delle lernee con alcune forme di copepodi parassiti (Dichelesthium, Caligus, Argulus, ) in quanto che egli le riunì con questi in una tribù speciale quella degli Armwiirmer e poneva le forme di lernee comprese nel suo genere Anops coi generi di sifonostomi.

De Blainville c'informa, che un anno o due prima, nel i8 14 , egli pure era stato indotto a riconoscere la necessità del separare le lernee in generi dif. ferenti; e che fu persuaso ad assegnare loro un posto tra gli Entomozoa, o animali articolati, riguardandole, « come un gruppo anomalo di vermi, intermedio tra i suoi Heteropoda e Tetradecapoda. » Al pari di Oken egli aveva fatto conoscere la relazione che evidentemente esiste tra loro e i Caligidae, ma non ancora inclinava a riferirle ai crostacei. Questo suo modo di vedere pubblicò nel 1816 , nel suo «Prodr. de Classification Nouvelle du Règne Anim.» e si deve ritenerlo personale a meno che non si fosse inspirato a quanto Oken aveva detto precedentemente. In un lavoro ulteriore dedicato specialmente alle lernee, Blainville ricercò anzi la prova diretta della loro natura di crostacei.

Wiegmann, anche appoggiato dal1' autorità di altri autori, ad es. di A. Nitzsch e del vecchio Leuckart, nel suo «Grundriss der Zoologie, 》 1832, le riunì ai Siphonostomi di Latreille in uno e medesimo ordine da lui distinto come Parassita.

Cuvier, il fondatore dell'anatomia comparata, di già nella prima edizione del suo celebrato lavoro, « Règne Animal, » aveva riconosciuti alcuni generi di copepodi parassiti come crostacei. Tosto, dopochè egli aveva pubblicato detto libro, il fisico Surriray, fece 1'importante scoperta che le uova erano contenute in lunghi filamenti sospesi all'addome, e che i giovani, quando nascono, non sono somiglianti ai loro parenti, ma al contrario sono estremamente simili a giovani Cyclops. De Blainville ricorda il fatto (Journal de Physique, I822), nel suo eccellente articolo « Lernaea, » e ammette pienamente la verità della scoperta di Surriray; e rileva l'affinità tra i caligidi e i lerneidi. Egli tuttavia mantenne questi ultimi fra 
gli Epizoa. Desmarest, infine nelle sue "Consid. Gén. sur la Classe des Crustacées 》 I 825, p. 343, note; sembra essere stato il primo a riferire le lernee, come un gruppo, alla classe Crustacea.

Per stabilire e confermare in modo definitivo questa unione bisognava sempre più avere una prova ulteriore strettamente scientifica, e questa fu fornita dalle pazienti ricerche di Alessandro Nordmann.

Nessun naturalista ha contribuito così efficacemente a farci conoscere la vita dei copepodi parassiti quanto Alessandro Nordmann professore di Zoologia e di botanica nel Liceo Richelieu di Odessa, che nel 1832 pubblicò le celebri 《Mikrographische Beiträge » dove è esposto il risultato delle sue ricerche sopra la generazione e lo sviluppo delle lernee, che ebbero d'allora in poi un posto ben definito nella classe dei crostacei. Da quell' epoca data il principio di un periodo di studi veramente scientifici sui copepodi, che rivelarono gli stretti rapporti di parentela tra le lernee e gli altri sifonostomi e fecero fare un passo notevole alla conoscenza della morfologia e della sistematica di tutto l'ordine.

«La prova che un Caligus è legato con una Lernaea per evidenti forme intermedie, scrive Gerstaecker (op. cit. p. 596), dovette col tempo involontariamente condurre anche alla supposizione dell'esistenza di rapporti di affinità tra i caligidi e $\mathrm{i}$ ciclopidi più simili a seconda della loro complessiva formazione del corpo.... La scoperta del Nordmann di giovani foggiati a forma di entomostraci, l'osservazione della loro evoluzione a traverso stadi ciclopiformi nell'Achtheres e nel Tracheliastes, la conoscenza fatta in essi di paia di membra boccali e di antenne per quanto molto piccole, e in singoli casi (Pennella) anche di paia di piedi natatori rudimentali, provò luminosamente che appartengono all'ordine dei copepodi anche quelle forme dall'aspetto piì degradato dal parassitismo e di natura vermicolare ».

Del resto il lavoro di Nordmann apri anche un nuovo orizzonte circa i rapporti fino allora non ravvisati e raramente apparenti tra le femmine e i cosidetti maschi pigmei nei sifonostomi e specialmente nelle lernee; e per una serie di osservazioni istruttive venne arricchita parimente la conoscenza dei generie delle specie circa l'anatomia interna, i rapporti ecologici dei parassiti rispetto ai loro ospiti. Il progresso sviluppatosi collo studio sui copepodi dopo la comparsa dell'opera di Nordmann fu oltremodo notevole e rapido secondo le direzioni più svariate.

Non finirei tanto presto se volessi riassumere anche brevemente tutti i lavori diversi divolgati negli anni seguenti, basterà farne un rapidissimo cenno.

Nell'anno r839, Rathke pubblicò le sue osservazioni importanti sull'anatomia del Dichelesthium sturionis, e ciò poco tempo dopo che Pickering e Dana avessero èdito una interessante monografia su di un Caligus (della quale sarà fatta menzione più sotto a proposito dei caligidi). In pari tempo (1839) v. Siebold scopriva nel Cyclopsine castor (copepode libero) la fecondazione della femmina per opera degli spermatofori applicati ai lati dell'anello genitale dal maschio, processo riscontrato pure per le forme parassitarie. 
In breve sopratutto gli studi di Nordmann mossero un gran numero di osservatori come ad es. Burmeister (1835), Kol1ar (I835), Johnston (1835-36) ed Enrico Kröyer (1837-38), quasi in pari tempo, ad esaminare i più svariati pesci per ricercarvi i loro rispettivi parassiti, ed a portare maggiore luce sulla cognizione di generi nuovi e specie, altrettanto numerosi quanto in parte diversi e con aspetti molto strani.

Milne Edwards, nel I 840, pubblicava la sua importante opera sui crostacei, e per primo compose un sistema di classificazione naturale per i copepodi parassiti, al quale più tardi fecero seguito altri tentativi di disposizione metodica per opera di Thorell (I86I) e di Steenstrup e Lütken (I86I).

Dopo la scoperta fatta da Audouin e da Milne Edwards (1826) del Nicothoë astaci sul gambero comune, parassita foggiato in modo tutto proprio, si trovò che $i$ pesci non erano $i$ soli ospiti di tali forme ma che altri animali ben più bassi specialmente le ascidie, gli anellidi, gli echinodermi, le pennatule, il Balanoglossus e la Doris potevano albergare copepodi. Una serie numerosa di naturalisti illustrarono chi una chi molte specie nuove di tale gruppo di parassiti.

Non essendo questi. crostacei compresi in quelli da me studiati, basterà che citi i nomi degli autori che se ne occuparono per ordine di data delle loro pubblicazioni: Will (I 844), Allman (I 847), Leydig ( I 853), Thorell (I 859) (I 862-68), Sars (I86 I), Keferstein (1863), Boeck (1860) (I86I), v. Bruzelius (I858), Hancock (I863), Mayer, Della Valle e Giesbrecht con lavori di recente pubblicati.

Con tali opere la conoscenza di tutta una serie di forme e in pari tempo dei rapporti ecologici dei copepodi ha progredito di molto; ugualmente le nuove cognizioni acquistate sulla struttura intima di molte nuove specie non mancavano di esercitare un'influenza essenziale sulle vedute di quel tempo a proposito della sistematica; e contribuirono sopratutto a far rinunziare alla separazione fissa, fino allora conservata, dei copepodi liberi dai sifonostomi di Latreille. La parentela tra i due gruppi già intraveduta da Burmeister ( 1837 ), sebbene più tardi da lui negata (I843, p. 319), fu resa manifesta col lavoro di W. Zenker (') che ebbe il merito di avere riconosciuto fra le due divisioni l' unità del tipo nella costruzione del corpo e di avere riferite le differenze delle parti boccali al modo diverso di vivere.

Numerosissimi sono i naturalisti che si rivolsero allo studio dei copepodi parassiti dei pesci in questi ultimi cinquanta anni. Tale è la copia del materiale bibliografico da non potersi certamente riassumere nelle presenti pagine.

I sifonostomi in genere e le lernee formarono l'oggetto di lavori in modo speciale ricchi di notizie o di figure da parte di Dana (1853) che pubblicò un'opera poderosa sui crostacei degli Stati Uniti; da parte di Steenstrup e Lütken (186I), di Kröyer (1863), di Nordmann (1864), di Heller (1865), il quale illustrò un materiale ricchissimo di crostacei raccolti durante il viaggio della nave austriaca « Novara. 》

Oltre a ciò furono fatte conoscere in parte molti generi e specie nuove, in parte singole forme, da Van Beneden (1850-60), Kölliker (I852), Gerstaecker

(1) Zenkr:R W. - Ueber die Cyclopiden des süssen Wassers: Wiegmann's Archiv. f. Naturgesch. XX, I, p. 88-102, Taf. 6, 1854 . 
(1853-54) Pagenstecker (r861), Turner e Wilson (1862), Steenstrup (1862), Bergsoe (I864), Hesse, Schaub e altri.

Brüh1 (r860) fece la scoperta morfologicamente importante della presenza di numerose, per quanto estremamente piccole, paia di piedi bifidi della Lernaeocera. Carlo Claus, morto pochi anni or sono, professore a Vienna, si distinse in sommo grado per studi notevoli e numerosi compiuti sui copepodi, pubblicando memorie che fanno epoca. Le sue osservazioni cominciate dopo il i 857 fruttarono una serie ricchissima di pubblicazioni sulla morfologia e anatomia di questi esseri. Egli è stato il degno continuatore dell'opera celebre di Nordmann. Sopratutto importanti sono le memorie seguenti: "Ueber den Bau und die Entwickelungsgeschichte Parasitischer Crustaceen », I858; "Ueber die Familie der Lernäen, » I86I; "Ueber den Bau und die Entwickelung von Achtheres percarum, » I86x; «Beobachtungen über Lernaeocera, Peniculus und Lernaea, I 868.

Di molto interesse è stata la scoperta fatta da Metzger nel r868 dei maschi delle lernee. Egli inoltre trovò le forme giovanili della Lemaea branchialis tra le branchie di Platessa flesus Cuv., mentre la femmina adulta vive su pesce di specie diversa. Le sue ricerche e quelle di Schimkevitsch, di Wierzeijski ma sopratutto quelle recenti del russo Pedascenko portarono un largo contributo alla conoscenza dello sviluppo della Lernaea. Nel 1877 Wierzeijski ha pubblicato una memoria colla quale fa conoscere che la Pennella varians presenterebbe le stesse abitudini della Lernaea branchialis, poichè passerebbe la prima parte della sua esistenza parassitaria sulle branchie delle seppie e dei calamari, per trasportarsi in seguito sul delfino dove trascorre il periodo della riproduzione.

Gerstaecker finalmente ha riassunto in un voluminoso trattato sui crostacei (1866-1879) quanto si conosce fino ai suoi tempi sulla classe di questi artropodi e segnatamente dei copepodi. Egli ha distribuito le famiglie secondo concetti moderni di sistematica e dà la chiave per la conoscenza dei generi.

I.' ordinamento sistematico di Gerstaecker in parte è quello stesso che Claus stabiliva nel $1862,(')$ soltanto modificato e migliorato. Egli divise $i$ copepodi in I6 famiglie; ed a capo del sistema pose quelli adatti per la vita libera e alla fine della serie quelli più pregiudicati nelle loro funzioni animali per causa del parassitismo. Intermedie sono le forme di passaggio tra la vita libera e quella parassitaria. Il sistema risponde perfettamente alle vedute odierne della scienza circa l'adattamento degli animali all'ambiente e alla loro evoluzione. Difatti l'autore aveva in mente componendo un simile prospetto sistematico di tutto l'ordine dei copepodi, di ristabilire in qualche modo la strada e i gradini per cui la natura ha tentato di arrivare, per lento processo graduale, dalle forme libere (Pontellidae e Calanidae) a quelle conformate per un parassitismo temporaneo (Corycreidae e Notodelphyidae) e da queste finalmente ai veri parassiti.

Gerstaecker abbozzava così il concetto della lenta e continuata degradazione delle funzioni animali in opposizione a quelle vegetative, causata dal modo di vivere dei nostri copepodi.

(1) Claus C. - Untersucbungen über die Organisalion und Verwandschaft der Copepoden: Würzburger naturwissensch. Zeitschr. III, p. 5I-IO3. 
Questa idea geniale non ha potuto realizzarsi che in parte, come lo confessa l'autore stesso, perchè è andato incontro alle difficoltà, che sopravvengono sempre in ogni tentativo di sistematica. Se pure nei copepodi si presentino i passaggi di forma e del modo di vita, questi non sembra abbiano seguito una direzione sola, piuttosto, come pare, partono da un punto comune verso due o parecchi lati e col loro percorso capriccioso, producono svariate combinazioni. Per questo fu impossibile al Gerstaecker di distribuire le famiglie in una serie: egli tentò tuttavia di presentare uno schema arborescente rispondente il meglio possibile a tutti i rapporti di parentela fra $i$ copepodi. La difficoltà aumentava col fatto che a quel tempo i copepodi semi-parassiti indicanti il passaggio dalle forme libere a quelle parassite, erano ancora poco noti. Sebbene gli studi ulteriori non sembrino avere accresciuto di molto la loro conoscenza, tuttavia non possono modificare per nulla questo suo sistema, il quale è l'unico che si fonda sopra caratteri naturali.

Per quanto concerne i copepodi parassiti, nel sistema fondato da Gerstaecker e nella distribuzione dei generi, che seguirò anch'io, la posizione sistematica di un certo numero di copepodi rimane ancora incerta perchè fondata sulla conoscenza della sola femmina, ma col tempo, nuovi studi e altre ricerche embriologiche o la scoperta dei maschi, potranno fare scomparire le poche incertezze che ancora sussistono, e migliorando il sistema, permetteranno di vedere distribuite tutte le forme dei crostacei parassiti a seconda delle loro affinità naturali. Questo quadro sarà il compimento dell'abbozzo già intraveduto e delineato, così magistralmente, da questo autore.

In questi ultimi tempi, l'Italia può vantare di aver avuto un illustratore distinto dei copepodi nel compianto Sebastiano Richiardi, dell'Università di Pisa, che si occupò con intelligenza di copepodi parassiti di cui raccolse un materiale ricchissimo e che disgraziatamente fin qui illustrava solo in piccola parte. Egli istitui la famiglia dei Philicthyidae, della quale egli descrisse 19 specie. Dell'opera sua a questo riguardo dirò più avanti. Oltre al catalogo delle specie italiane pubblicò non pochi altri lavori originali sopra nuove specie di copepodi lerneidi (v. Bibliografia).

In pari tempo Antonio Valle pubblicava in varie riprese, 1'elenco dei copepodi parassiti dei pesci viventi nell'Adriatico, il che fece pure lo Stossich col « Prospetto della Fauna del Mare Adriatico». Prima di loro già Cornalia, nel 1865, s'era segnalato per uno studio pregiato sulla Lophoura (Rebelula).

Questi autori italiani ora citati si occuparono di specie per la massima parte spettanti alla nostra fauna. Anche lo scrivente di recente ha pubblicato alcune note rilevando la presenza di un certo numero di copepodi parassiti dei pesci, nel mare ligure e in quello dell'isola d'Elba.

Se l'opera di questi pochi italiani contribuì in certo qual modo a farci conoscere la distribuzione geografica di questi animali nei nostri mari, non è stato questo un merito a loro esclusivo. Anche distinti naturalisti stranieri avevano fatte per lo innanzi ricerche nel Mediterraneo e nell'Adriatico, ma non mai di proposito. Sebbene accurate fossero state le indagini da parte di Heller, Hope, Claus, Carus, Kurz, Heider, Schaub, Hartmann, i loro lavori si restrinsero unicamente ad alcune specie, e la lontananza di essi dal mare e la difficoltà di procurarsi 
grande copia di pesci, a loro non permisero di estendere le investigazioni ad un numero maggiore di tali rappresentanti della nostra fauna. Ciò nondimeno Victor Carus, valendosi dei lavori dei sopracitati autori ci ha dato nel suo «Prodr. faun. medit. » 1885 , un elenco abbastanza completo delle forme viventi nei nostri mari. aggiungendo una diagnosi succinta e l'habitat di ciascuna specie.

\section{Caligidi.}

Fin qui abbiamo trattato dei copepodi parassiti detti lerneiformi (appartenenti alle famiglie Dichelesthiidae, Lernaeidae, Chondracanthidae, Lernaeopodidae) che offrono fra loro qualche comunanza di forma per una maggiore influenza in loro esercitata dal parassitismo che li ha ridotti a forme affatto strane e alle volte bizzarre.

In questa seconda parte trattiamo separatamente della storia dei caligidi, perchè formano una divisione a sè distinta di copepodi foggiati più o meno secondo un tipo unico, nei quali il parassitismo non è venuto che in parte a modificare la primitiva forma di copepode libero. Essi hanno ancora qualche somiglianza colla tipica forma di crostaceo, perchè portano ancora chiaramente impressa, anche allo stato adulto, nel loro corpo, l'impronta di un Cyclops. Sistematicamente parlando detti animali harno un posto, nella scala zoologica, più elevato che non i copepodi lerneini. Per questo avrei dovuto occuparmene da principio, nella prima parte, ma siccome costituiscono un gruppo assai meno numeroso e meno svariato nelle forme, che il precedente, e perchè sotto l'aspetto del parassitismo, ripeto, sono meno notevoli, così ho creduto conveniente dar loro un posto secondario in questi cenni storici.

Gli autori non sono certi se Aristotile abbia conosciuto alcuna forma di caligide, sebbene in qualche punto della sua «Hist. Anim.» accenni indubbiamente ad animali parassiti dei pesci. Gerstaecker, di cui abbiamo sopra fatto menzione, perchè autore del miglior compendio sui copepodi che si conosca, ritiene che i

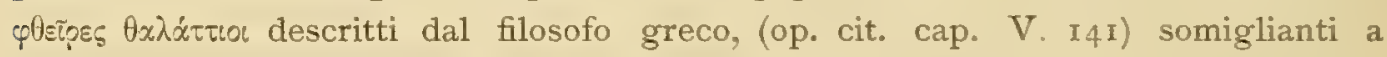
cloportidi, debbansi prendere per isopodi parassiti ma non già come Caligus; invece dubita assai a proposito dell' oũoțos (op. cit. cap. VIII, I28) se Aristotile abbia voluto accennare ad un caligide (Cecrops) oppure ad una Cymothoa (isopodo parassita). Più sopra ho manifestata la mia opinione in accordo con quella di Steenstrup e Lütken, nel ritenere che l'oĩotpos non sia altro che la Brachiella Thynni.

Dobbiamo venire a tempi molto più vicini a noi per vedere ricordata con certezza qualche forma di caligide. Linneo sembra sia il primo autore che ne faccia cenno. Nella sua «Fauna Suecica», 2 ediz., r761, descrive brevemente una specie che vive sopra il salmone e il merluzzo, e propria del mare norvegese. La ricorda col nome di Monoculus piscinus. Nella medesima opera egli ci presenta un'altra specie di pidocchio che fu trovata sopra il salmone e che egli chiama Pediculus farionis. Secondo Baird (') (British Entomostraca, 1850, p. 257) tali

(1) Nel redigere queste notizie storiche mi sono valso in parte, per quanto riguarda i primi tempi, della eccellente pubblicazione di questo autore inglese; pp. 257-260. 
forme indicateci con due nomi diversi non sarebbero che una sola specie, o per lo meno due tipi molto affini di Caligus: e questo si desume dalla descrizione generale di detti piccoli animali, entrambi organizzati ugualmente e caratterizzati specialmente dal possedere essi due lunghi fili ovarici. Anche dal fatto d'essere stati tutti due trovati sulla stessa specie di pesce, Baird ne deduce chiaramente 1'affinità loro che già Linneo stesso, nel $I 767$ ( 12 ediz. Syst. Nat.), aveva intraveduta, e Gmelin nella edizione del i788, di nuovo confermata.

Linneo non è il solo che abbia, in quei tempi, citato e descritto copepodi di questa famiglia. Nel i $762 \mathrm{Ström}$ descrisse e ci diede una figura di due parassiti che Baird (op. cit.) ritiene pure per Caligus, forse soltanto il maschio e la femmina della stessa specie, a cui fu dato parimente il nome di Pediculi marini o pidocchi dei pesci.

Anche Baster nella sua «Opuscula Subseciva, » 1765 , diede la descrizione e 1a figura di due o tre specie diverse di questo genere, accompagnandole con molti dettagli. L'autore ha rappresentato il corpo di questi animali colla testa in giù e scambiò i due lunghi fili ovarici per antenne, prendendo cioè la coda per la testa. Queste figure e la descrizione di Baster sembrano avere ingannato anche Linneo; non ostante che prima avesse di già descritto l' animale correttamente nella sua «Fauna Suecica, » egli di poi, nella 12 ediz. del suo «Syst. Nat.», I 767 , descrisse i1 Monoculus piscinus, costituendo due specie tra loro diverse, (i due sessi) 1'una per avere due antenne più lunghe che il corpo, e l'altra essendo affatto sprovvista.

Ström di nuovo, nel I 770, descrisse un' altra specie di Caligus ed evidentemente anch'egli cadde nell' errore di scambiare la parte caudale per quella cefalica.

Linneo nella sua I 2.2 ediz. del «Syst. Nat.» confuse 1'Argulus col Caitgrus. Nella «Fauna Suecica» egli aveva descritto quelli come specie separate; ma nel suo ultimo lavoro non solamente riportò la sua propria precedente descrizione dell' Argulus, come corrispondente al gen. Caligus, ma egli anzi aggiunse la figura data da Loefling per questo parassita, come una prova evidente della loro identità. Questa erronea sinonimia fu ripetuta da Fabricio «Systema Entomologiae, " 1775, e da Gmelim «Systema Naturae», I 788. Tuttavia S1abber, I778, precedentemente all' edizione di Gmelin, nel suo lavoro « Naturkundige Verlustigingen » diede una figura col nome di Oniscus lutósus di un caligide, delineando le vere antenne e qualche altra parte con molta correttezza.

Spetta ad O. Fabricius e ad Herbst il merito di avere meglio compresa l'anatomia dei crostacei riferentisi a questa famiglia. Le specie da loro descritte, nella «Fauna Groenlandica, » I780, dal primo, e nella «Berlin Gesellschaft Skrifter, » I 780 e I 782 , dal secondo, sono accompagnate, specialmente le ultime, da molti dettagli; e furono illustrate assai accuratamente.

M üller, nel suo «Prodr. Zool. Danicae», i 776 , introdusse per le forme finora menzionate l'appellazione di Binocolus, adottando questo nome da Geoffroy; ma nel suo «Entomostraca », I785, egli fondò il genere Caligus.

Fin qui nessun naturalista aveva accertato chiaramente la posizione degli occhi nei caligidi; ed è l'apparente mancanza di organi visivi o meglio la supposta cecità di questi animali, che aveva suggerito a Müller il nome generico ora menzionato. Questo naturalista, sebbene realmente osservasse i veri occhi, non li considerò tuttavia come tali, e li confuse completamente con altro organo. Non ostante questa 
svista, la descrizione del genere e delle due specie che egli ci dà, è la migliore di qualunque altra dei suoi tempi.

Geoffroy descrisse e figurò, nel suo «Insectes de Paris», I 764 , un piccolo crostaceo sotto il nome di Binocolus hemisphaericus, il quale presenta una certa somiglianza con un Caligus, e Linneo nel «Syst. Nat.» $12 .^{a}$ ed., lo citò come un sinonimo pel suo Monocolus piscinus. Questa sinonimia è ripetuta da Fabricio nel suo lavoro di già citato: "Entom. Syst." 1793, e nel Suppl. di questo lavoro (I798). Tuttavia Baird (op. cit.) ci fa osservare che questo piccolo crostaceo non è un Caligus, ma un genere completamente differente, al quale Latreille ha dato il nome di Prosopistoma.

Cuvier menzionò il (Calygus) Caligus nel suo «Tableau Elément. " I798; e Latreille nel suo «Hist. Nat. Crust. et Ins.», 1802, illustrando estesamente questo genere, e ricordandocene i caratteri già noti per opera di Müller e di altri.

Ris so nel suo "Hist. Nat. des Crust. des Environs de Nice », I8 I6, e nel suo «Hist. Nat. de 1'Europe Mérid. » 1826, parimente citò diverse specie riferentisi ai Caligidae.

Sempre secondo Baird, Tilesius in una pubblicazione "Mém. de l'Acad. Imp. des Scien. de St. Pétersbourg », 1815, riportando una lunga descrizione delle due specie di Müller, aggiunse qualche notizia su di un certo numero di altri animali stretti da parentela coi caligidi, nei quali credette attribuire in parte la causa del fenomeno della luminosità del mare; e Lamark, nella prima ediz. del suo «Hist. Nat. des Anim. sans Vertéb., 18I8, descrisse brevemente le poche specie precedentemente già note. Il genere intanto veniva citato e descritto in Inghilterra da Leach nell' articolo: «Crustaceology » pubblicato nell" « Edinburgh Encyclopaedia », 1814; e in quell'altro «Annulosa» nel «Suppl. Encyc. Britann. », 1816: più tardi nel capitolo «Entomostraca» del «Dict. Sc. Nat. » I8Ig. Poi Otto (1828), Burmeister (I831), Kröyer (1838-39), Milne Ediwards (I833, Ann. Sc. Nat.; e 1840), ci hanno dato sui caligidi una grande quantità di notizie estremamente interessanti a riguardo dei costumi loro e specialmente in ordine alla sistematica.

In America Pickering e Dana hanno studiato con somma cura una specie di Caligus trovata colà abbondantemente sul comune merluzzo delle loro coste, e pubblicarono una monografia importante della specie, nell' « Americ. Journ. of Science ", I838. Tale lavoro fece fare un rapido progresso alla conoscenza della morfologia nonchè dell' anatomia interna dei Caligzıs. Ma il loro studio contemplò solo la forma adulta e per lungo tempo non si conobbe che questa. Baird stesso nel I850, quantunque compendiasse, mostrando di conoscerle, quasi tutte le forme di copepodi parassiti noti fino a' suoi tempi, e propri della fauna inglese, tuttavia diede prova di ignorare la struttura dei giovani caligidi.

La forma Chalimus, presentata nella memoria di Burmeister, I83I, è difatti considerata come genere distinto, mentre non è che una forma corrispondente ad uno stadio giovanile del gen. Caligus, come rilevarono Stein (1852) ed Hesse più tardi. E merito di Goodsir «Edin. Philos. Journal», I842, e degli antori ora citati di avere scoperto e fatto conoscere le fasi naupliane e larvali ulteriori di questo genere. Però Groodsir mostrava di ignorare ancora la natura della forma Chalimus. 
Non solo Baird (I850), già ricordato, ma anche Dana (1853) diede un grande impulso alla conoscenza dei caligidi, ed altri autori più tardi, specialmente Steenstrup e Lütken (186r), Kröyer (I863) ed Heller coi loro classici lavori, rivelarono agli studiosi un numero grande di generi e di specie.

Accanto a questi nomi illustri è dovere menzionare qui $\mathrm{Hesse}$, van Ben eden, Gerstaecker, Olsson, i quali contribuirono non poco a renderci note varie specie isolate. Degno di ricordo è C. B. Wils on che illustrò recentemente (I g05) i caligidi della fauna degli Stati Uniti in un ben riuscito lavoro.

Fra tanti autori stranieri pur troppo nessun zoologo italiano (') prima dell'ultimo trentennio, ci diede notizie di alcuno dei rappresentanti di questa famiglia (seppure alcuni abbiano solo descritto copepodi lerneidi). A mala pena Verany (I846), per la fauna del Genovesato, ne aveva citato soltanto due forme (Caligus Rissoamus e Cecrops Latreillii). Per contro, in questi ultimi anni, troviamo Richiardi che citò i nomi, nel suo Catalogo (1880), di una serie numerosa di caligidi appartenenti alla nostra fauna. Anche Valle, Stossich e lo scrivente elencarono buon numero di questi parassiti per varie località d'Italia, ma evidentemente $\mathrm{i}$ loro studi furono ristretti ad alcune parti soltanto dei nostri mari ed ebbero più che tutto importanza faunistica.

Sotto questo punto di vista è degna di menzione l'opera di Carus, che più sopra ho citato, il quale valendosi delle pubblicazioni italiane precedenti, e di quelle di Heller e di altri, pubblicò un compendio riassuntivo, un Prodromus della fauna del Mediterraneo, presentando tutti i caligidi conosciuti fino al 1885 .

\section{Filictidi.}

Nello stesso modo che ho dovuto separare i caligidi dai copepodi lerneiformi nel tesserne la loro storia, perchè sono animali fra loro notevolmente diversi, così pure, ora, sono costretto a parlare, in un capitolo a parte, dei Philicthyidae che parimente costituiscono un gruppo indipendente. Essi possono considerarsi come copepodi tipici, affini alle forme ancestrali, formanti una famiglia non legata per rapporti diretti di parentela con quelle finora studiate o per lo meno coi copepodi più degradati dal parassitismo. Per la semplicità della loro organizzazione, per la regolarità della segmentazione osservata nei maschi, pel debole dimorfismo sessuale tra maschio e giovane femmina, almeno nel Leposphilus labrei, questa famiglia sarebbe piuttosto congiunta coi copepodi superiori, ma la sua posizione sistematica non è stata ancora bene accertata nè è ora compito mio di determinarla.

Per un altro motivo ancora sono stato indotto a trattare separatamente dei filictidi. I rappresentanti di questa famiglia sono stati studiati per la maggior parte dal nostro compianto Richiardi, e perciò mi premeva rilevare, in un paragrafo speciale il merito ch' egli ebbe nel farci conoscere tali copepodi parassiti. Si deve a lui

(1) Cornalia e Costa descrissero bensi forme di copepodi parassiti ma non caligidi, il Costa figuró soltanto una Nemesis che apparticne ai Dichelesthürlae. 
se questa famiglia, una delle ultime introdotte nella classe dei crostacei, è diventata in breve così ricca di specie.

Il Prof. Leydig, nel 1850, trovò per caso sulla Corvina nigra, nei canali mucipari della testa, il primo filictide, che chiamò Sphaerosoma (nome che Richiardi cambiò in quello di Sphaerifer, essendo stato impiegato già da Leach per un genere di coleotteri), e fu il primo a darne una descrizione piuttosto incompleta , che lasciava cosi desiderare altre notizie intorno alla storia di questo elegante parassita.

Dopo di Leydig nessun altro zoologo si occupò più, per lo spazio di venticinque anni, di questo crostaceo. Le notizie che in questo tempo si aveva di esso erano così incomplete che non se ne conobbe il sesso, nè il numero, nè la forma delle appendici articolate delle quali è fornita la testa, per cui non fu possibile stabilirne le affinità, e collocarlo convenientemente nei quadri sistematici della classe dei crostacei, ed anzi v. Beneden credette dovesse essere ascritto alla famiglia delle Hirudineae presso il suo genere Histriobdella, ed era persuaso che lo studio dello sviluppo avrebbe confermato l'esattezza della sua opinione e di tale ravvicinamento. (') Sebbene Leydig avesse già affermato che questo crostaceo potesse comprendersi tra le lernee, questo suo modo di vedere non ebbe seguaci. Richiardi soltanto venne a persuadere $i$ naturalisti sulla vera natura di copepode offertaci dal suddetto filictide, pubblicando, nel $\times 874$, un importante studio su altri esemplari della medesima forma. Fece allora notare che non poteva essere conservato tra le lernee, ma che costituiva un genere affine ai Chondracanthus ed intui fin d'allora che i Philichthys presentavano caratteri. sufficienti per formare con quello una famiglia distinta. Richiardi più tardi scoperse e studiò altre specie sia di Sphaerifer sia di Philichthys, ne rilevò gli stadi larvali di Nauplizus e sempre più si convinse della indipendenza dei filictidi dalle altre famiglie.

La scoperta e la fondazione del genere Philichthys è più recente di quell'altra forma sopra ricordata. Steenstrup nel r86I ne studiò un esemplare raccolto nei seni frontali di Xiphias gladius, e un anno dopo ne scoprì anche la forma maschile. Egli dapprima dubbioso se dovesse riferirlo agli anellidi, colla scoperta del maschio, in cui riconobbe la forma di copepode, cambiò opinione e l'avvicinò ai crostacei.

Bergsoe in un suo viaggio in Italia, si occupò dello studio di questo parassita, che trovò comunissimo. Ma Heller ancora nel I865 manteneva ancora qualche dubbio sulla natura dei Philichthys che tuttavia collocava fra i Chondracanthidae.

Fino allora il genere non era rappresentato che da una sola specie: ma Richiardi tosto ne scoperse molte nuove e avendo studiato i maschi e le forme di Nauplizus e rilevato le affinità di queste forme cogli Sphaeripher, tolse ogni dubbio sulla loro vera natura e indicò il posto distinto dei filictidi nei quadri sistematici. Vide anche la necessità di scindere il gen. Philichthys in due generi e propose il nome nuovo di Polyrrhynchus per un gran numero di essi.

Noi seguendo le vedute del Richiardi in questo lavoro, nella Sistematica, abbiamo considerato i filictidi come una famiglia ben distinta. Al Richiardi spetta il merito d'aver chiaramente espresso, fin dal 1874 , il suo pensiero sulla neces-

(1) P J. Van Beneden. - Les commensaux et les parasites dans le rếgne animal. Paris, I875, p. 74. 
sità di separare questi copepodi dagli altri in una speciale famiglia. In quel tempo egli così scriveva: "nel ricercare le affinità di moltissime specie di crostacei parassiti non si deve tener conto solo delle forme di uno dei due sessi, ma di entrambi, ed in questo caso i maschi dei Philichthys, che conservano pressochè tutti i caratteri dei copepodi liberi, e subiscono pochi cambiamenti in confronto di quelli dei Chondracanthus, danno al genere una tale incontrastabile superiorità, da non potere essere collocato in una stessa famiglia con questi ultimi, ma da costituire una propria, nella quale dovranno probabilmente prendere posto tutte le specie che vivono nei tubi e seni cosi detti mucipari dei pesci $\gg\left({ }^{1}\right)$.

Le ulteriori osservazioni di Vogt (1877) e di Claus (1887) e specialmente quelle recentissime da parte di A. Quidor (I906) provano. la verità di questa affermazione. Questo autore in particolare, ha riconosciuto che $\mathrm{i}$ filictidi per il loro capo, per i cinque segmenti del torace e per i cinque segmenti addominali sono forme tipiche a cui spetta un posto piuttosto elevato nella sistematica dei copepodi, posto che però rimane ancora da stabilirsi esattamente.

(1) Richiardi S. - Sopra lo Splaerifer cornutus etc. Atti Soc. Tosc. Sc. Nat in Pisa, Vol. II, fasc. 2, I874, p. 12 .

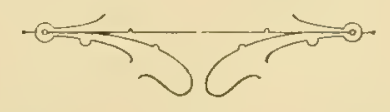




\title{
SISTEMA TICA. \\ CATALOGO DEI COPEPODI PARASSITI STATI RISCONTRATI \\ SUI PESCI D'ITALIA.
}

\author{
Sistema di classificazione delle famiglie appartenenti all' Ordine dei Copepodi \\ secondo Gerstaecker (I87I).
}

Pontellidae

2. Calanidae

9. Corycaeidae

10. Ergasilidae

3. Harpactidae

4. Peltidiidae

I

II. Ascomyzontidae

6. Notodelphyidae

7. Ascidicolidae

8. Buproridae

| commensali

semi-parassiti

I 2. Caligidae

(Philichthyidae)

13. Dichelesthiidae

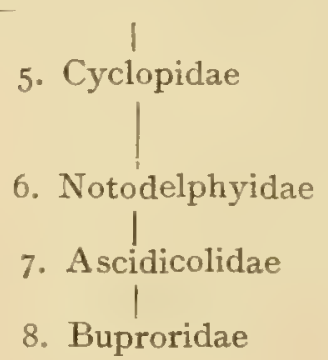

liberi

(

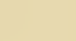

14. Lernaeidae

I5. Chondracanthidae

r6. Lernaeopodidae

parassiti

Secondo questo sistema, elaborato con un concetto moderno e scientifico, ho elencato i copepodi parassiti dei pesci nelle pagine seguenti. A tale serie di famiglie è da aggiungersi oggi quella dei Philichthyidae dei quali tuttavia resta ancora da determinarsi esattamente la posizione sistematica. Ritenendo questi ultimi piuttosto affini ai caligidi che non ai copepodi lerneidi propriamente detti, ho assegnato loro un posto tra quella famiglia e l'altra dei Dichelesthiidae. Rimangono inoltre da includere nel prospetto di Gerstaecker, per quanto riguarda le forme parassitarie viventi su altri animali fuorchè i pesci, le nuove famiglie scoperte di recente, Coniostomatidae, Herpyllobiidae e Monstrillidae, che hanno caratteri loro propri; ma anche per esse la posizione sistematica come già per i filictidi, non è stata ancora stabilita in modo esatto e non è compito mio di occuparmene qui. Come si vede i copepodi sifonostomi dell' antico sistema di Milne Edwards, che corrispondono a quelli viventi sui pesci, i quali fanno l'oggetto del presente 
lavoro, si trovano distribuiti nel sistema di Gerstaecker, nelle ultime cinque famiglie (vedi N. 12, I3, I4, I5, 16). Non ho creduto opportuno di occuparmi delle forme semi-parassite viventi sui tunicati e su ospiti pelagici, perchè avrei dovuto allargare il campo delle mie ricerche e non sarei riuscito certamente in poco tempo ad avere a mia disposizione il materiale occorrente per rendere il lavoro completo. Unicamente perchè viventi anch' essi sopra i pesci ho dovuto trattare di varie specie dei generi Ergasilus, Bomolochus ed Eucanthus seppure appartenenti alla famiglia Ergasilidae, (vedi N. Io) costituita da forme di passaggio dai copepodi liberi a quelli parassiti.

\section{Fam. I. - ERGASILIDAE.}

Gen. Eucanthus Claus,

Eucanthus Balistae CLAUS, $q$ e $\sigma$.

Eucanthus Balistae Claus, I864 (8) Tav. XXXVI, fig. 2 t-27.

- - Richiardi, i880 (9) p. I48.

- - CARUS, I885 (2) p. 353.

Habitat. - Claus notò questa forma, che descrisse nel $\mathbf{1} 864$, pel Mediterraneo (? Messina), sulle branchie di un Balistes sp.; Richiardi sulle branchie di Balistes capriscus Linn.

\section{Eucanthus Marchesetti Valle,}

Eucanthus Marchesetti VALLE, 1884 (5) p. I.

$$
\text { -- } \quad \text { - Carus, } 1885 \text { (2) p. } 354 \text {. }
$$

Habitat. - Valle riscontrò la presenza di questa nuova specie abbastanza frequente sopra le branchie della Motella tricirrata Block nell'Adriatico. Egli la nominò senza descriverla.

Gen. Pseudoeucanthus n.

Ho chiamato questo $n$. gen. con tale nome per la falsa somiglianza che presenta col gen. Eucanthus.

Carattere peculiare del nuovo genere si trova nella forma dei piedi mascellari posteriori, che, come negli Eucanthus presentano un grande uncino falciforme, però a differenza di quelli, sono situati dal lato esterno dell' apparato boccale e non all' indietro. Di più mentre in quel genere il ramo esterno del quarto paio di piedi è allungato a forma di uncino, nella nostra forma questa struttura speciale manca, tale ramo è laminare, munito di setole e non diverso per ciò da quello delle paia precedenti. Nelle restanti parti la nuova forma presenta rapporti di affinità col gen. Bomolochus, ma più ancora col surriferito Eucanthus. Il suo posto è, sistematicamente parlando, tra i Lichomolgidae accanto al gen. Terebellicola ed Eucanthus, come verrà meglio precisato col seguente specchietto: 


\section{Sub fam. LICHOMOLGIDAE.}

Apertura della bocca arvicinata all' origine dellc antenne.

Picdi del $40^{\circ}$ paio ugualmente formati di due rami come i precedenti.

Ramo interno del $40^{\circ}$ paio di piedi ugualmente formato di tre articuli come nelle paia precedenti. Antenne anteriori alla base non allargate.

Costituite di sei segmenti, le posteriori di tre segmenti..... . Terebellicola Sans Costituite di quattro segmenti; i piedi mascellari posteriori con uncino terminale a forma di falce, e disposti all'indictro dell'apparato boccale; ramo esterno del $4{ }^{\circ}$ paio di piedi allungato a forma di uncino

Eucanthus CL:Ls

Costiluite di qualtro segmenti; $i$ piedi mascellari posteriori con uncino terminule a forma di falce, e disposti esternamente ai lali dell'apparajo loccale; ramo esterno del t.0 paio di piedli non uncinato tha provvedulo di due selole all' estremita. . Pseudoeucanthus n. gen.

Pseudoeucanthus Alosae n. sp., \& e 8 . Tav. XI, fig. I- 8.

Eucaulluus Alosas Brian, 1902 (5) P. 33.

Questa nuova forma ha il tipo di un Bomolochus, però se ne distingue per avere il cefalotorace meno ampio e i segmenti successivi più simili fra loro. Tanto il cefalotorace quanto i segmenti addominali sono poco convessi alla loro superficie dorsale: quello ha forma ovale, mentre l'addome è allungato e il suo primo segmento è fuso col cefalotorace, e si presenta costituito da cinque articoli decrescenti alquanto in larghezza e in lunghezza dall'avanti all' indietro. Il post-addome è più stretto e va sempre più attenuandosi verso l'estremità posteriore; consta di tre segmenti, all'ultimo dei quali stanno fissate due appendici di forma rettangolare allungata, tendente all' ovale e provvedute di una lunga setola per ciascuna lamina e di quattro altre più brevi, disposte secondo la fig. I.

I sacchi oviferi sono grossi e di forma ovale allungata (fig. 2). Le uova pure sono relativamente grosse, disposte in due righe, e ne ho contate dalle $1_{5}$ alle I $_{7}$ per ciascun sacco ovifero in un esemplare; in un altro fino a 37.

Dimensioni:

Lunghezza totale del corpo della femmina sino all' estremità delle setole caudali . . . . mm. I,08

Lunghezza del solo cefalotorace . . . . . »0,34

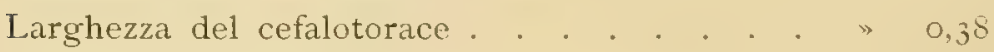

Lunghezza dei sacchi oviferi, variabile fra . . $\$ 0,50-0,60$

Larghezza massima degli stessi...... (.) 0,10-0,16

Le antenne anteriori sembrano formate da quattro articoli; 1'articolo basale discretamente ampio e allungato, è provvisto anteriormente di setole piumate piuttosto larghe alla loro base, in numero di una quindicina circa: assai tenui e corti sono gli altri articuli che vanno sempre più decrescendo man mano che s'allontanano dalla base e l'ultimo termina all'estremità con un ciuffo di setoline di cui una specialmente mostrasi più sviluppata.

Il margine anteriore del cefalotorace non è inciso nel mezzo; presenta invece al di sotto della fronte due uncinetti rivolti all'indietro, caratteristici nei Bomolochus. 
Le antenne del secondo paio (fig. 4), situate a breve distanza dal margine frontale e dietro gli uncinetti anzidetti, constano di due articoli, uno basale discretamente grosso, e un altro ripiegato sopra lo stesso, sul lato esterno, il quale presenta l'estremità libera divisa in parecchie spine o uncini e in due appendici ciliate al margine. Queste ultime sembrano essere prolungamenti dello stesso margine laterale interno del secondo articolo, il quale è pure munito di piccole cilia o dentelli disposti in due o tre linee, per tutta la sua lunghezza.

La bocca è circondata da una cornice di listelli chitinici che danno ad essa un aspetto complicato. Si vedono diretti verso il suo centro dapprima due stiletti chitinici, che sono le mandibole (fig. 3), e al di sotto due altri stiletti bi-articolati con una larga base triangolare, ossia i piedi mascellari del primo paio. Questi sono muniti di setole brevissime sul loro articolo terminale. Tra le dette due paia di membra, ossia tra le mandibole e i piedi mascellari si vede interposto un paio di pezzi chitinici trasversali, ciascuno dei quali sembra formare un cerchietto alla estremità, e dentro questo sembrano penetrare le punte degli stessi piedi mascellari.

Caratteristico è il paio di piedi mascellari del secondo paio (fig. I), che sono situati ai lati della bocca e alquanto più in alto di essa, e offrono un articolo basale ampio e lungo ed un uncino falciforme con direzione dall'avanti verso l'indietro e colla punta rivolta verso il centro del cefalotorace.

Il primo paio di piedi (fig. 5) è costituito da una piccola lamina basale al cui margine inferiore è inserita una lamina più larga, ossia il ramo interno indistintamente tri-articolato, e che porta sei setole piumate sul margine inferiore. Un'altra setola è inserita più verso 1 'interno di questa piccola lamina basale. Invece ìl ramo esterno trovasi fissato ad un lato della lamina basale e disposto in modo da formare un angolo retto col ramo interno: esso porta altre sei setole piumate. Questo paio di piedi è più o meno simile a quello di altri Bomolochus.

Ll secondo paio di piedi (fig. 6) è foggiato più normalmente secondo la forma ti pica: somiglia di più ai piedi natatori dei copepodi viventi liberamente. Il ramo esterno è tri-articolato e porta tre setole all'estremità, decrescenti in lunghezza dall'esterno all'interno. I due rami natatori sono piuttosto uguali in lunghezza. L'interno è pure tri-articolato e, se ho potuto veder bene, porta tre setole sul margine interno, una sul primo articolo e due sul secondo; il terzo articolo ne presenta tre o quattro, tutte piumate.

Il terzo paio di piedi (fig. 7) consta, come i precedenti, di due rami. Le setole nel ramo esterno, all' estremità, sono soltanto in numero di tre e crescenti di lunghezza dall' interno all' esterno. Gli articoli di questo ramo sul margine esterno, tanto in questo paio come nel secondo e nel quarto, appariscono provvisti di peli. Il ramo interno è abbastanza più piccolo, più breve, e porta per ogni articolo, dal lato interiore, una setola, e sull' estremità, tre o quattro altre.

Il quarto paio di piedi (fig. 8) è foggiato come gli altri, consta di un articolo basale piuttosto allungato e di due rami con tre articoli. Il numero delle setole però è diverso. Il ramo esterno ne porta tre all' estremità e il ramo interno solo due, ma un' altra s'osserva sul primo e sul secondo articolo in quest' ultimo ramo, dal lato interno. Il ramo interno è poi abbastanza più piccolo e tenue dell'altro. Anche il margine esteriore del ramo interno è munito di peli, e la presenza di questi si nota 
anche nelle paia precedenti, ma più che tutto nel ramo esterno sul lato o margine esteriore.

Habitat. - Parecchi esemplari trovati dal D. Damiani fissati all'occhio della Clupea alosa Cuv., a Portoferraio il i7 Febbraio rgoo e il ig Aprile Igor.

\section{Gen. Bomolochus Nordmann,}

Bomolochus Muraenae Richiard, \& Tav. XII, fig. I-9.

Bomolochus Miraenae RICHIARDi, 1880 (9) p. 147.

1 - $\quad$ - Carus, 1885 (2) p. 353.

- $\quad$ - Brian, 1903 (9) P. 178.

Descrizione della femmina.

Questa forma non era ancora stata descritta ma soltanto nominata dal Richiardi.

Visto dorsalmente il cefalotorace (fig I) si presenta assai convesso e il suo margine frontale è intero, anteriormente circolare, il margine posteriore è pressochè dritto e abbastanza largo. Il secondo segmento piuttosto breve mantiene all' incirca la larghezza del cefalotorace, così pure il terzo segmento. Più stretto invece è il quarto segmento addominale, il quale presenta il margine posteriore arrotondato e porta alquanto nascosto, al di sotto, il quinto segmento, il meno vistoso di tutti, assai breve e un po' meno largo del quarto segmento: ai lati esso porta il quinto paio di piedi rudimentali. A questo segue il segmento genitale sempre più ristretto, (fig. 2), di poco. più largo che lungo e infine quattro segmenti maggiormente e progressivamente attenuati ai lati, costituenti il post-addome, terminato da due laminette caudali con setole assai sviluppate.

Questi quattro segmenti del post-addome hanno il margine posteriore come ciliato o meglio armato da spine o dentelli disposti in linea sul limite posteriore di ciascun segmento, ed anche le due appendici caudali presentano un certo numero di queste spine o cilia sul lato posteriore.

Prima di passare alla descrizione delle rispettive appendici riunisco qui sotto le diverse misure da me prese sul corpo di questo Bomolochus:

Dimensioni: La lunghezza totale della femmina, comprese le setole caudali, varia da mm. 2,24 a mm. 2,50, e senza setole da I, 74 a I,80 mm. La larghezza verso il margine posteriore del cefalotorace è pressochè di o, $70 \mathrm{~mm}$. La larghezza del quarto segmento di mm. o, 40.

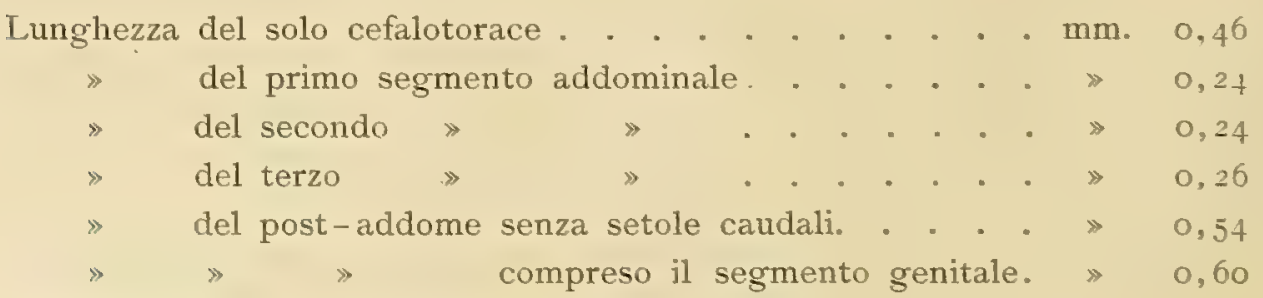

Lunghezza della setola caudale più lunga, variabile da mm. 0,50 a 0,70 


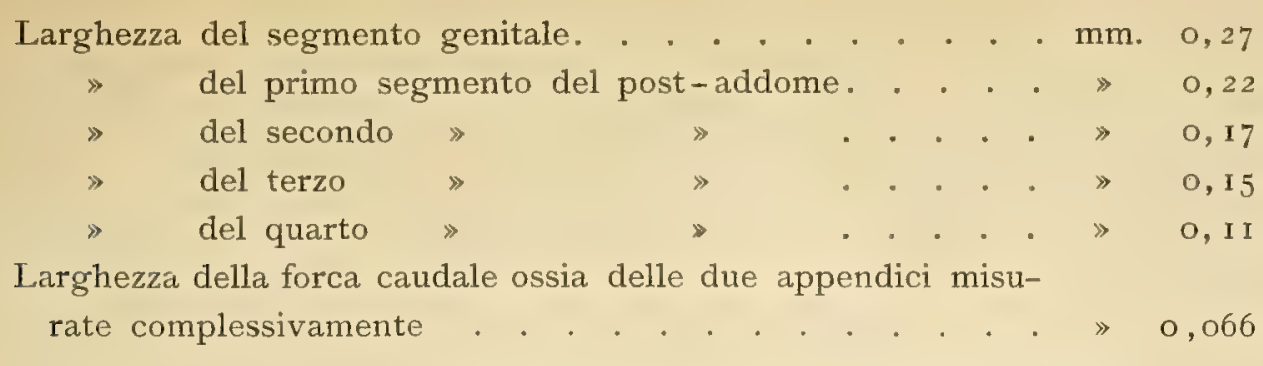

Appendici. - Le antenne anteriori (fig. 4) sono formate di quattro articoli e si presentano armate di setole e di spine: queste ultime soltanto in numero di due o tre pel primo articolo basale. Le setole sono piumate, larghe e brevi verso la base, più sottili e lunghe verso l'estremità libera delle antenne.

Le antenne posteriori (fig. 5) sono composte di tre articoli e l' ultimo porta due appendici ottuse all'estremità e pelose (come peloso è pure tutto l'articolo) e un ciuffo di peli o spine adunche, di cui mi sembrò poterne contare quattro o cinque.

Le mandibole (fig. 3) sono date da due stiletti bj-dentati all'estremità che s'avanzano liberamente verso la linea mediana longitudinale e si toccano colle loro punte. Le mascelle come nel gen. Bomolochus sono rudimentali e rappresentate da una lamina chitinica con un palpo e tre grandi setole piumate. Due altri stiletti più sottili, allungati e bi-dentati all' estremità, costituiscono i piedi mascellari anteriori. Inferiormente fanno seguito quelli del secondo paio, rappresentati da due grosse lamine allungate e piegate verso 1'interno, nella estremità superiore toccandosi fra loro, e portano, sulla parte concava di questa loro curvatura anteriore, un filamento e un dentello per ciascun piede.

Il primo paio di piedi (fig. 6) è rappresentato da una lamina piccola, stretta, a cui stanno inseriti due larghi rami natatori, 1' uno fissato sul lato esterno, 1'altro verso il

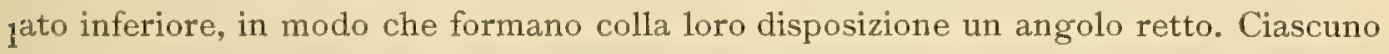
dei rami porta traccia di una primitiva articolazione, in quello esterno contansi nove larghe e brevi setole piumate, in quello interno otto; e queste ultime vanno decrescendo in lunghezza dall'interno all' esterno. Un' altra setola trovasi fissata alla lamina basale più verso 1'interno, da ogni lato di un comune pezzo basale mediano. Col segmento basale del primo paio di piedi è connesso un vero apparato chitinico di sostegno, costituito da liste e da pezzi insieme congiunti nel modo più svariato e caratteristico e di cui solo il disegno potrà dare un'idea.

Il secondo paio di piedi (fig. 7) non differisce dalla forma solita propria del genere. Si notano due rami tri-articolati fissati ad una lamina basale per ognuno dei piedi. Il ramo esterno porta dal lato esteriore cinque spine ossia una su ciascuno dei due primi articoli e tre sull'ultimo, il quale per giunta porta ancora sei setole piumate. Anche il secondo articolo sul lato interno presenta una lunga setola piumata. Il ramo interno presenta dal lato esterno tre spine, una sul secondo e due sull'ultimo articolo, ove pure si notano quattro setole. Tre altre di queste ultime, stanno distribuite eziandio sul lato interno del primo e secondo articolo.

Anche il terzo paio di piedi è foggiato presso a poco come il secondo. Il ramo esterno tri-articolato presenta dal lato esteriore quattro spine, e sette setole distribuite, in parte sull'estremità e in parte sul lato interno. Sul ramo interno si contano 
tre spine dal lato esterno, distribuite sul secondo e terzo articolo: quest' ultimo porta soltanto tre setole sull' estremità, e fra di esse ho notato pure, se non erro, una quarta spina. Altre tre setole disposte sul primo e sul secondo articolo si vedono dal lato esterno di questo stesso ramo notatorio.

Il ramo interno del quarto paio di piedi (fig. 8) porta soltanto due setole e due spine sull'ultimo articolo, altre due setole dal lato interno, distribuite sul primo e secondo articolo. Sull' altro ramo notansi cinque spine dal lato esterno e sei setole fissate all' estremità dell' ultimo articolo, mentre una sola sul lato interno e fissata sul secondo articolo.

Il quinto paio di piedi (fig. 9) è costituito da una lamina ovale piuttosto circolare, fissata ad un breve segmento basale inserito sul quinto segmento addominale. Il margine esterno di questa lamina è guarnito di tre setole e il contorno è ciliato.

Ognuna delle appendici caudali porta quattro setole: la più interna è tre volte più lunga della seconda che gli sta da presso. Questa a sua volta è almeno tre volte più lunga delle altre due situate un po' più all'esterno dell'appendice caudale stessa. In un individuo la piủ lunga setola caudale raggiungeva sino mm. o, 7 I (ossia era assai più lunga del post-addome e dell'anello genitale, che insieme riuniti misurano appena $\mathrm{mm}, 0,60$.) In questo medesimo esemplare la seconda setola non arrivava che a $\mathrm{mm} .0,27$.

Habitat. - Questa specie fu trovata per la prima volta da Richiardi sulle branchie della Muraena helena Linn. Indi il D.r Damiani, il 24 Dicembre Igo2, ne raccolse parecchi esemplari sullo stesso ospite a Portoferraio e, in grazia dell'invio fattomene, ho potuto descriverla, poichè Richiardi l'aveva soltanto nominata.

Bomolochus unicilrus RichIARDi, $q$.

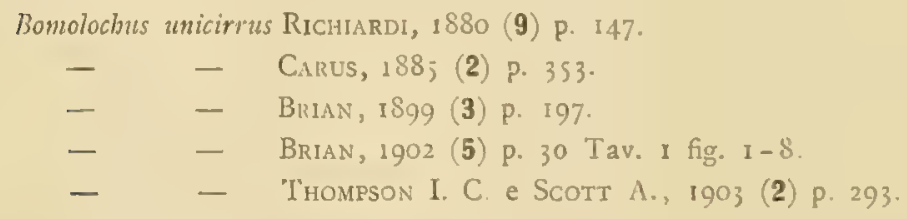

Habitat. - Richiardi rinvenne questo copepode sulle branchie della Lichia glauca Linn. e della L. amia Linn. nel Mediterraneo. Ho esaminato due esemplari femminili inviatimi nel I 899 da Portoferraio dal D. ${ }^{r}$ Damiani e raccolti da lui nella cavità branchiale di Lichia glauca Linn. Dapprima avevo soltanto citato questa specie (1899), ma siccome era stata semplicemente nominata dal Richiardi, ho creduto opportuno più tardi di descriverla (I902). Altri esemplari, che riferisco ad essa, ho raccolto io stesso a Napoli, il 3 Agosto I903, sulla Sphyraena vulgaris Cuv. e Val.

Bomolochus Belones Burmeister, $q$ e $\sigma^{7}$.

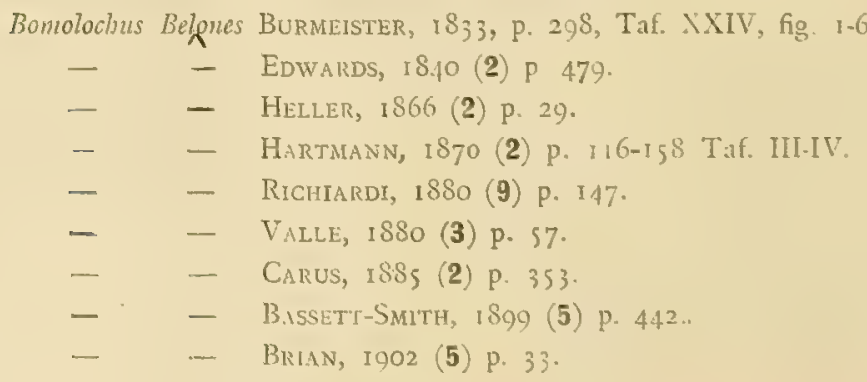


Habitat. - I primi esemplari furono trovati sulle branchie di un Belone vulgaris Flem. (Esox Belone Linn.) dal D.r Stannius (studiati poi da Burmeister) presso l'isola d'Helgoland nel mare del Nord. Hartmann ha poi esaminato altri esemplari provenienti da Nizza, da Trieste e da Venezia e rinvenuti sulle branchie di Belone vulgaris Flem. Sul Belone rostrata Fab. tale parassita è citato da Heller e da Valle parimente per l'Adriatrico; Valle anzi lo dice comune in quel mare. Nel Mediterraneo fu di nuovo riscontrato da Richiardi sulle branchie di Belone acus Risso sul quale ospite fu raccolto anche dal D. Damiani a Portoferraio e da me a Napoli. Carus indica per questa specie anche la località di Borkum 1'isola ben nota del mare del Nord.

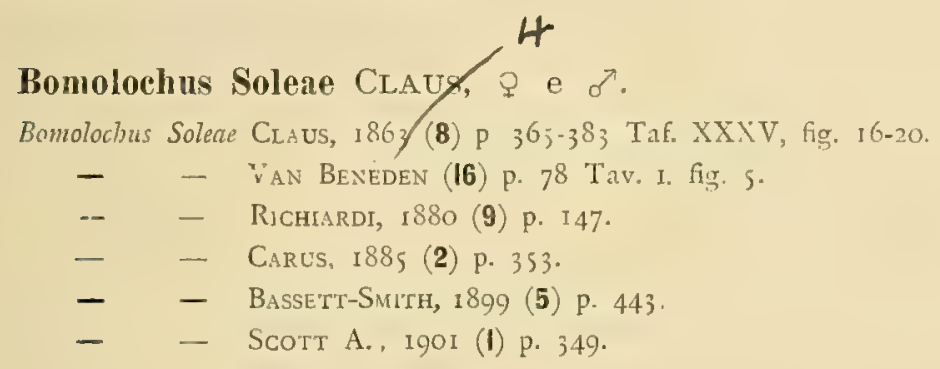

Habitat. - Claus scopri questa specie fissata alle branchie della Solea vulgaris Cuv, sulle coste dell' isola d' Helgoland (numerosi esemplari). Per le coste del Belgio fu più tardi indicata da Van Beneden che forse ne ignorava la scoperta dapprima fattane da Claus e, a caso, la chiamò collo stesso nome. Ne trovò due soli esemplari femminili carichi d'uova, su di un individuo di Solea vulgaris Cuv. che non aveva più di 9 cent. di lunghezza, al principio di Maggio. Richiardi la rinvenne pure nel Mediterraneo sulle branchie di detto pesce.

\section{Bomolochus cornutus Claus,}

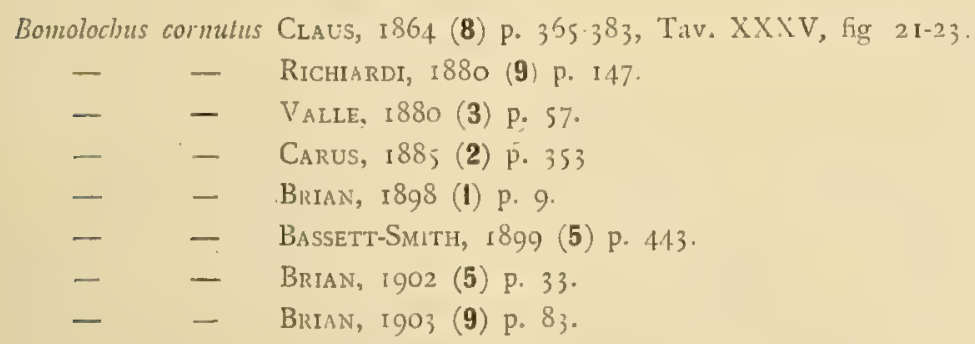

Habitat. - Claus rinvenne per la prima volta questa specie che descrisse, sulle branchie del raro Asterodermus coryphnenoides (stad. giov. di Luvarus imperialis Raf.) in Messina. Gli esemplari da lui osservati avevano $3 \mathrm{~mm}$. di lunghezza. Richiardi poi la riscontrò parassita sulle branchie di altri pesci: Astrodermus elegans Risso, Exocatus volitans Linn., Sayris Camperi Lac. e Chupea sardina Risso. Valle la trovò frequente sulle branchie della Clupea papalina Bp. Io la citai per Genova sulle branchie dello Scomberesox Rondeleti C. V., e ne ebbi varie volte esemplari da Portoferraio dove il D. Damiani li raccolse nel cavo branchiale di Sayris Camperi Lac., e tra le branchie di Chupea sardina Cuv. e sulla mucosa dell'opercolo di Exocotus Rondeletii C. V. E specie comune. 


\section{Bomolochus minimus RICHIARDI,}

Bomolochus minimus RicHIARDI, 1880 (9) p. 147.

- - Carus, i88s (2) p. 353.

Specie non descritta. Richiardi la rinvenne sulle branchie di Serranus scriba Linn. nel Mediterraneo.

\section{Bomolochus oblongus RICHIARDI,}

Bomolochus oblongus Richiardi, i 880 (9) p. 147

- - Carus, r885 (2) p. 353.

Specie non descritta. Fu raccolta da Richiardi sulle branchie dell'Oblata melamura Linn. nel Mediterraneo.

\section{Gen. Bomolochus NordmanN,}

\section{Sub genus Anchistrotos $n$.}

Antenne anteriori poco allargate alla base, mancanti della curvatura ad angolo retto che è caratteristica del gen. Bomolochus: costituite da 5 articoli (considerando il segmento basale diviso in due), i due primi articoli allungati. I piedi mascellari inferiori hanno una posizione normale e sono provveduti di due prolungamenti sottili, allungati e diretti all'indietro. Cefalotorace munito, presso i lati del margine frontale, di due hamuli come negli Eucanthus. Ricordando la presenza di questi uncini ho dato a questo sottogenere il nome greco di Anchistrotos (armato di uncini).

Anchistrotos Gobii n. sp., $\nmid$ e $\delta$. Tav. XIII, fig. r-ro.

Descrizione della femmina.

Lunghezza del corpo circa mm. I $1 / 2$. Il cefalotorace (fig. 3) ha quasi forma circolare; una piccola salienza rotondeggiante si nota in mezzo del margine frontale. Seguono a questa porzione quattro segmenti leggermente decrescenti in larghezza dall'avanti all'indietro e un altro di forma piuttosto globoso e alquanto più largo e più lungo dei precedenti, che costituisce il segmento genitale. Altri quattro segmenti gradatamente più stretti, man mano che s'avvicinano all' estremità posteriore, costituiscono il post-addome, terminato all'indietro da due piccole laminette caudali, rettangolari, munite di due lunghe setole per ciascuna, l'una del doppio più lunga dell'altra, e da altre tre brevi setoline.

Presso a poco uguale come nel gen Taeniacanthus di Sumpf è la disposizione delle parti boccali sulla superficie inferiore del cefalotorace: come in quel genere, e come pure nel gen. Encanthus si nota, anche nella nostra forma, la presenza di due uncini corrispondenti agli hamuli dei caligidi, situati presso il margine laterale del cefalotorace da una parte e dall'altra, e un po' al di sotto delle antenne del primo paio. Queste sono lunghe, costituite da sei articoli, i due primi saldati insieme costituiscono l'ampio segmento basale il quale presenta molte setole sul margine anteriore (circa 20 setole). 
Le antenne del secondo paio (fig. 6) sono formate da due articoli, e l'ultimo porta all' estremità quattro lunghe spine leggermente ricurve verso l'interno e un' appendice ciliata da uno dei lati.

L'apparato boccale è costituito da un paio di mandibole foggiate a guisa di stiletti che s'avanzano verso l'interno e si toccano reciprocamente colla loro estremità che sembra bifida. Seguono le mascelle rudimentali rappresentate da alcuni filamenti piumati inseriti su un pezzo basale comune, e al di sotto, il primo paio di piedi mascellari costituiti da un doppio stiletto, piccolo ed inserito su di un segmento basale, stretto ed allungato: infine dietro a questi ultimi, notasi il secondo paio di piedi mascellari, formato da un largo pezzo chitinico basale a cui sono inseriti, in una delle estremità, due filamenti allungati diretti all' indietro (fig. 4).

Il primo paio di piedi, inserito sul margine inferiore del cefalotorace, è dato da una lamina basale a cui sono fissati due altri rami in forma di lamine piatte, munite di setole.

Su ognuno dei primi tre segmenti addominali liberi, dal lato ventrale, è fissato un paio di piedi natatori formato di due rami bene sviluppati; ogni piede è congiunto col suo opposto per mezzo di una sottile lamina mediana. Ogni ramo conta tre articoli e ogni articoló è munito di setole e di spine come l'indica la figura 9 e 10.

Il quinto paio di piedi è dato da un'appendice stretta ed allungata fissata per ogni lato del suo proprio segmento addominale. Questa appendice porta tre setole brevi sull' estremità ed un' altra sul margine esterno.

L'orifizio genitale è posto su ciascun lato del sesto segmento del corpo ed è formato di una fessura protetta all'intorno da listelli chitinici disposti in modo speciale a guisa di cornice, e da tre lunghe setole che originansi dit un piccolo tubercolo, rudimenti rappresentanti il sesto paio di piedi.

Dimensioni :

Lunghezza totale del corpo, variabile fra. . . . . . mm. $1,4 \ldots \ldots$

" delle setole caudali ... . . . . 》 0,24

» del post-addome . . . . . . . . . \$ 0,34

》del cefalotorace. . . . . . . . . " 0,44

Larghezza dello stesso . . . . . . . . . . . . » 0,50

Lunghezza di tutti i segmenti addominali liberi, compreso l'anello genitale. . . . . . . . \$ 0,36

\dei sacchi oviferi, variabile fra.... . $0,84-1,00$

Spessore degli stessi, circa........ . . ". 0,13

Il maschio (fig. 1, 2, 7-10, ̀̀ pressochè uguale alla femmina ora descritta.

Habitat. - Sulle pareti della bocca di Gobius capito Cuv. e Va1.; molti esemplari raccolti da me a Napoli, il I 3 Luglio 1903. 


\section{Gen. Ergasilus NordmanN,}

Mrgasilus Sieboldii Nordmann, $q$.

Ergasilus Sieboldii Nordmanx, I832 (1) p. Is pl. I fig. I.

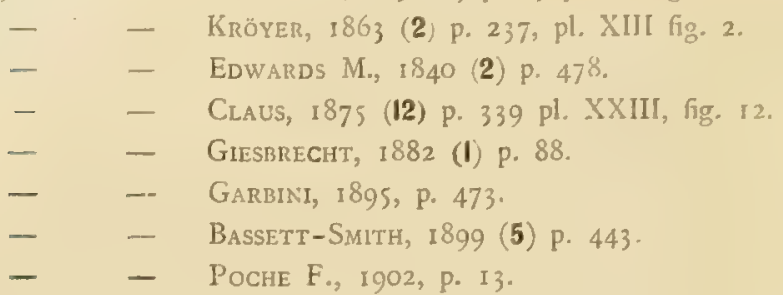

Habitat. - Comune e in abbondanza si rileva nelle acque dolci d'Europa sulle branchie di Esox lucius Linn., di Cyprinus carpio Linn., di Abramis brama Flem. e di Silurus glanis Linn. ecc. Garbini riscontrò la presenza di questo copepode in Italia, nel Veronese, sulle branchie di un Cyprinus.

A Monaco di Baviera osservai esemplari di detta specie raccolti da v. Siebold e conservati nell' Istituto Zoologico di quell' Universit . Erano accompagnati dall'indicazione seguente «br. Cypr. Erythrophth. Heilsberg." Da un accenno di Poche Franz si nota che Giesbrecht avrebbe indicato come ospite di questo copepode anche l'aringa (?).

\section{Ergasilus nanus BENEDEN v.,}

Ergasilus namus BENEDEN v., 1870 (16) p. 27 tab. 1, fig. 6.

- - Richiardi, 1880 (9) p. 147.

- - VAlle, I880 (3) p. 57 .

Habitat. - Richiardi citò questa specie pel Mediterraneo, da lui trovata sulle branchie di Mugil cephalus Cuv., M. capito Cuv., M. auratus Risso e M. salrens Risso. Valle la dice comunissima sulle branchie di Mugil saliens Risso, nell'Adriatico.

Fu questa specie scoperta per la prima volta da Van Beneden presso le coste del Belgio, parassita delle branchie di Mugil chelo Cuv. e da lui indicata inoltre per la località di Ostenda e per le coste di Bretagna. Il figlio di P. J. Van Beneden ne disegnò e pubblicò nell'opera "Les poissons des côtes de Belgique 》 una figura (Tav. I, fig. 6). Nella spiegazione della tavola vi è scritto che questa specie sarebbe parassita di Mugil capito Cuv., mentre nel testo è indicato come ospite il $M$. chelo Cuv.

\section{Fam. II. - CALIGIDAE.}

\section{Gen. Caligodes Heller,}

Caligodes laciniatus (KRöY.), $\&$.

(Chondracanthus lacinialus Kollar, nome di Museo.)

Scinenophilus laciniahus KRöYER, I86; (2) p. 153 , pl. VIII, fig. 3.

Caligodes lacinialus (Kr.) Heller, I 865 (I) p. ISo.

- $\quad$ - Brian, ig02 (5) p. 35

- - BASSETT - S.MITH, I 899 (5) P. 446.

- - POCHE Fro, Ig02, pag. I3. 
Distributio et habitat. - Kröyer indica per questa specie la località delle Molucche nelle Indie orientali, mentre Heller la nota nell'Oceano indiano quale parassita di una specie di Belone.

Io esaminai pochi e rari esemplari raccolti a Genova, nel Maggio I899, dal Sig. Borgioli, all' angolo boccale di Tylosurus (Belone) imperialis Raf. (= Belone Contrainii C. V.)

\section{Gen. Caligus MüLleR,}

Divisione I. Post-addome con un articolo solo.

Caligus curtus MüLL., $\&$ e $\sigma^{7}(?)$

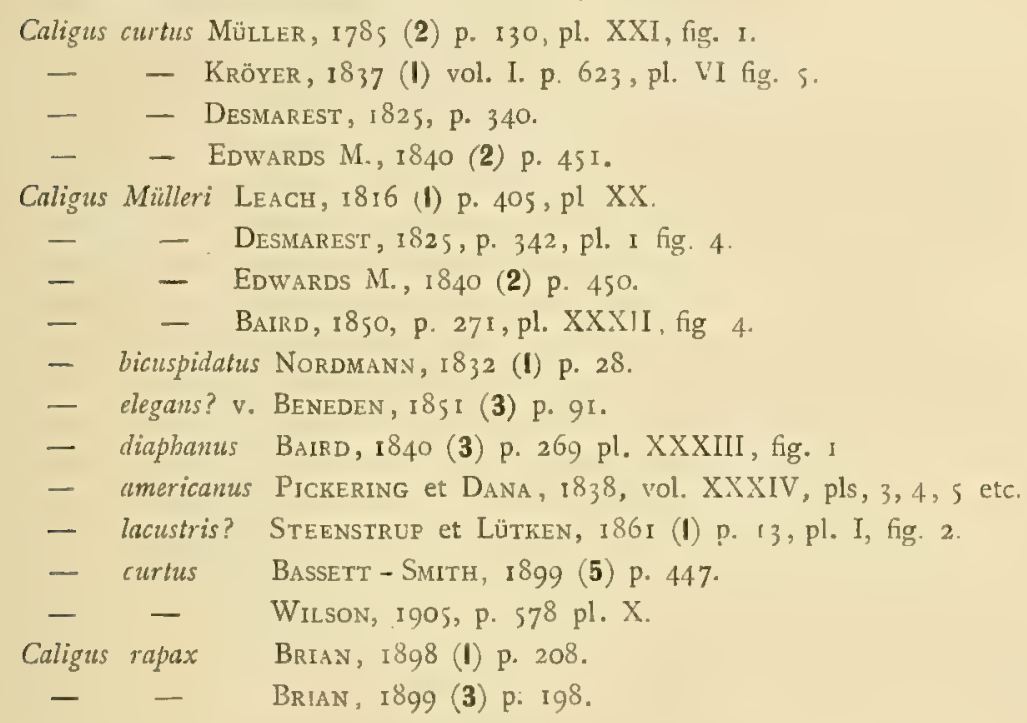

Distributio et habitat. - Questa specie, sotto il nome di Caligus Miulleri fu descritta da Baird, il quale indicò per questo copepode diversi ospiti, e come località il mare Britannico (Belfast Bay, Lough Neagh): gli ospiti che egli cita sono: Merlangus pollachius Cuv., Rhombus vulgaris Cuv., Merlangus vulgaris Cuv., Coregonus pollan Thomps., Gadus aeglefinus Linn., Mugil chelo Cuv.

Bassett-Smith ritiene come identico a questa specie il C. diaphanus di Baird, il quale autore ha descritto soltanto il maschio, e lo registrò come parassita, pel mare Britannico, sui pesci seguenti: Trigla pini Bloch, Lota molva Cuv., Merlangus carbonarius Flem., Pagellus centrodontus Cuv. e Val., Gadus morrhua Linn., Hippoglossus vulgaris Flem.

Milne Edwards non dà indicazioni d'habitat di questa specie, sia che egli la descriva come C.curtus, sia come C. Mülleri; soltanto parlando del C. amcricanus con cui sappiamo essere sinonimo, lo dice « trouvè sur la morue, dans les parages de Long-Island, Amérique septentrionale. " Non mi fu dato, non avendo potuto avere tra le mani i lavori originali, di conoscere se Müller, Kröyer, Desmarest, Leach, che descrissero pure questa specie, ora come C.curtus ora come C. Mïlleri, abbiano indicato 1'habitat di tale Caligus. Bassett-Smith, che ha passato in rassegna tutti i copepodi parassiti dei pesci in oggi conosciuti, indica soltanto come ospiti del C. curtus i seguenti: «Gadidae, Trigla spp., Rhombus maximus Cuv., Mugil, etc. » 
Gerstaecker nel suo classico trattato, alle forme di Caligus che Bassett-Smith ha ritenute per sinonime, ha assegnato ospiti diversi:

pel C. lacustris: Esox lucius Linn., Perca Auviatilis Linn., Leuciscus rutilus Linn.; pel C. Mïlleri: Merlangus pollachius Cuv. e M. vulgaris Cuv., Gadus aeglefinus Linn.

e G. morrhua Linn., Mugil chelo Cuv., Pleuronectes rhombus Linn.

e P. platessa Linn., Coregonus pollan Thomps.;

pel C. curtus: Merlangus vulgaris Cuv. e Gadus neglefinus Linn.;

pel C. elegans: Gadus morrhua Linn.;

pel C. americanus: Gadus morrhua Linn.

Quanto al Caligus bicuspidatus mancano i dati di località e di ospite. Nordmann notava fin dal 1832 pel C. Mülleri varie specie di Gadus, come segue: Gadus callarias (giovane di $G$. morrhua Linn.) e Gadus aeglefinus Linn.

Riscontrai la presenza di questa specie nel Mediterraneo. Sei esemplari femminili, mi furono inviati da Portoferraio dal D.r Damiani colà raccolti sulla mucosa boccale di Mugil cephalus Cuv., il I6 Giugno I899. Nelle mie precedenti pubblicazioni avevo riferito a torto a questa specie alcuni esemplari di Caligus tolti dalla Lichia amia Linn., esemplari che qui, più oltre, ho ritenuto come specie nuova e descritto col nome di C. Lichiae.

Due altri esemplari maschili di Caligus furono trovati a Grenova sul Mugil cephalus Cuv., l' 8 Novembre I889, ma sulla loro determinazione ho qualche dubbio.

La lunghezza da me osservata per le femmine è di $5 \frac{1}{2} \mathrm{~mm}$. Dei due maschi raccolti a Genova il più grosso misura $\mathrm{mm} .4 \frac{1}{2}$ di lunghezza.

\section{Caligus minimus Отто, $\nmid$}

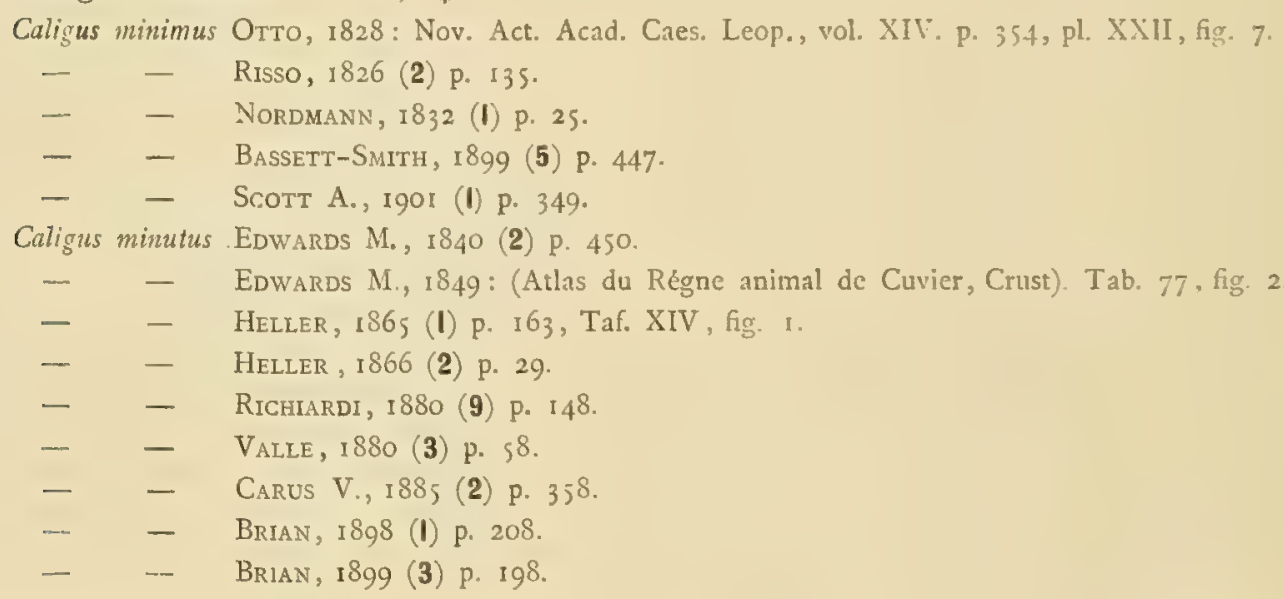

Distributio et habitat. - Questa specie ha per ospite il Labrax lupus Cuv., sul quale trovasi affissa per lo più sulle branchie o sulla mucosa della cavità boccale. Essa è citata da Heller, da Richiardi e da Valle pel Mediterraneo e per 1'Adriatico. Anche Risso l'indicò per la località di Nizza. Il D.r Damiani la raccolse a Portoferraio. A Genova la riscontrai fissata nella cavità branchiale del pesce sopra riferito. A questa specie di copepode dev' essere attribuito ancora un altro ospite, la Clupea finta Cuv., sopra cui fu rinvenuto un solo esemplare ritenuto prima 
da me per Caligus Gumardi Kröy., (I898 (I) p. 209), ma che con certezza ho riconosciuto appartenere alla specie C. minimus Otto.

\section{Caligus Trachypteri KRöXER,.}

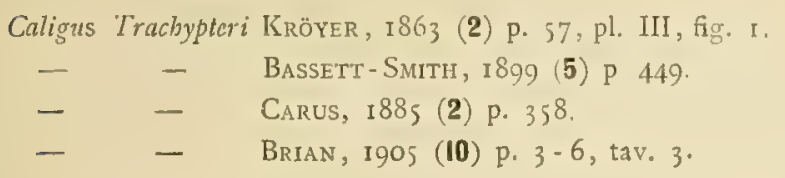

Distributio et habitat. - Ospite di questa specie è un Trachypterus sp. pescato presso i lidi di Sicilia (Mus. Caes. Vindob., Kröyer).

Ho trovato alcuni esemplari riferentisi a questa specie nel materiale di copepodi raccolti da O. G. Costa a Napoli nella prima metà del secolo XIX, e che si conserva nell' Istituto Zoologico della R. Università napoletana. Questi esemplari non avevano indicazione d'ospite, soltanto risultavano erroneamente determinati dal Costa per Notodelphys.

Caligus Alalongae Kröyer, $\sigma^{7}$.

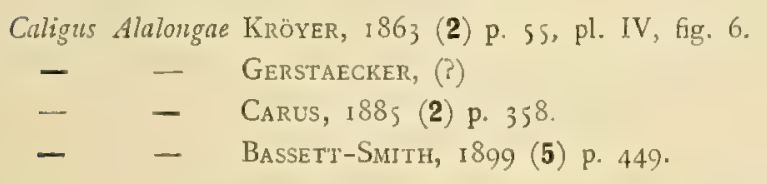

Habitat. - Kröyer descrisse soltanto il maschio di questo piccolo caligide da lui notato per l'Atlantico e di poi citato anche pel Mediterraneo da Gerstaecker e da Carus, sulle branchie di Thynnus alalonga Cuv. e Val.

? Caligus Lessonianus Risso (C. Lessonius), ơ e

$$
\begin{array}{cccc}
\text { Caligus Lessonianus Risso, } 1826 \text { (2) p. } 134 \\
- & - & \text { Carus, } 1885 \text { (2) p. } 359
\end{array}
$$

Habitat. - È specie stata trovata da Risso a Nizza sullo squalo Notidanus griseus Cuv.

Caligus Rissoanus M. Edwards, $\uparrow$.

Caligus Rissoanus LDWARDS M, , I 840 (2) p. 452.

$$
\text { - - Carus, } 1885 \text { (2) p. } 357 .
$$

Habitat. - Questo copepode fu trovato su di un pesce indeterminato a Nizza (Mus. Paris., H. Milne Edwards).

Caligus Lichiae n. sp. Tav. XIV, fig. I-I4. Tav. XVI fig. 8-II.

Caligus curlus BRIAN, 1898 (1) p. 208.
$-\quad-\quad$ BRIAN, 1899 (2) p. 4.

Descrizione della femmina (Tav. XIV):

Lunghezza $5 \%$-6 $\mathrm{mm}$. Lo scudo cefalico (fig. I) è di forma pressochè rotonda, convesso dorsalmente: i suoi lati vanno però restringendosi più sul davanti che sull'indietro. L'addome di forma quasi rettangolare colla maggior lunghezza nel 
senso longitudinale è tuttavia più ristretto verso il limite d'inserzione coll' anello toracico libero e va gradatamente allargandosi verso il lato posteriore il quale, visto dal dorso, presentasi incavato, mentre dal lato ventrale al posto dell'incavo fa vedere due lobi. Esso è di molto più ristretto dello scudo cefalico. La lunghezza sua, compreso anche l'anello toracico libero, è quasi uguale a quella del solo scudo cefalico. Assai più corto della metà circa, e più stretto è il post-addome (fig. I4) terminato da due piccole lamine caudali con quattro setole piumate su ciascuna.

Nella forma generale, eccezione fatta per le dimensioni, questa specie somiglia al $C$. isonix di Steenstrup e di Lütken, soltanto si rileva facilmente una differenza nella larghezza del corpo nel punto dove 1'addome si restringe per inserirsi coll' anello toracico libero, larghezza ben più rilevante nelia nostra forma. Se veniamo ai dettagli, se ne rilevano allora le spiccate differenze sia per la presenza, nella nostra specie, di un numero maggiore di setole nel secondo paio di piedi. di spinule e di denti nelle antenne del secondo paio e nei piedi mascellari, che mancano invece nel $C$. isonix (se è esatta la figura data dai sullodati autori); e specialmente per la presenza di un carattere singolare soltanto osservato finora nel Caligus della Lichia, consistente in un apparato particolare che giace sulla lamina del terzo paio di piedi, come più sotto sarà descritto. $E$ benchè possa parere a tutta prima che altre specie, ad es. il C.curtus, il C. rapax e il C. minutus somiglino nell' aspetto generale alla nostra forma e leggiere ne sieno le differenze, pure nel C. Lichiae vi sono caratteri così peculiari da non doversi ritenere altrimenti che come nuova specie.

Le antenne del primo paio (fig. 6) sono bi-articolate. Il secondo articolo (terminale) è sottile, allungato quasi più del doppio che il primo. Quest'ultimo è ricoperto sul margine superiore da numerose setole, il secondo ne porta un ciuffo nella sua estremità libera. Le lunule (fig. 6) sono abbastanza vistose, poste ai lati del largo margine frontale, di forma sub-tondeggiante.

Le antenne del secondo paio (fig. 7), disposte orizzontalmente sul lato ventrale dello scudo cefalico, sono potenti organi unciniformi tri-articolati, l' ultimo articolo foggiato ad uncino, più lungo degli altri. Il rostro boccale (fig. 4) è tozzo, un po' più lungo che largo, e ai lati presenta traccie di mascelle le cui estremità offrono un principio di biforcazione; ossia il ramo interno è appena abbozzato. Gli hamuli (fig. 3) presentano una base larga e hanno la punta, come al solito, rivolta all' indietro e ottusa.

La furcula sternalis (fig. 5) è a rami semplici poco divergenti, le sue estremità tendono a curvarsi alquanto verso l'interno.

Il primo paio di piedi (fig.9) è uni-ramoso; ogni piede consta di tre articoli, il primo breve e tozzo, il secondo più lungo, il terzo minore in grandezza. Mentre il primo porta una setola e una appendice (verosimilmente il rudimento del ramo natatorio interno), il terzo, alla sua estremità, porta invece tre pungiglioni più brevi e una lunga setola sull'angolo. La seconda e la terza di queste spine, quelle interposte tra la setola d'angolo e la spina esterna, hanno la loro estremità bi-dentata. Dal lato inferiore del terzo articolo si vedono tre lunghe setole piumate discretamente sviluppate. 
Il secondo paio di piedi (fig. Io) è invece bi-ramoso e ogni ramo consta di tre articoli. Il grosso segmento basale sostenente questi due rami è composto di due articoli, il primo brevissimo porta una setola piumata. Il secondo più grosso e lungo ha il margine inferiore finamente ciliato. Il ramo esterno tri-articolato presenta per ogni articolo una forte spinula ricurva all' esterno; il terzo articolo terminale per giunta oltre ad una setola rigida porta sei setole piumate, mentre il primo e il secondo articolo dal lato interno ne hanno una sola, e tutte queste setole crescono in lunghezza dall'esterno all'interno. Gli articoli del ramo interno sono pure muniti di setole distribuite in quest'ordine, sei per 1'articolo terzo, due per l'articolo secondo e una pel primo articolo e crescenti pure in lunghezza dall' esterno all' interno.

Il terzo paio di piedi natatori (fig. I I) è costituito da una larga lamina basale a cui è fissato ciascun piede da ogni lato, formato da due lamine bi-articolate munite di setole e di spine. Alla base del ramo esterno vi è per giunta fissata una potente spina uncinata ricurva, mentre l'articolo basale ha solo una setola piumata da una parte e una spina dall'altra; il secondo articolo, dal lato esterno, porta tre setole rigide o spine $\mathrm{e}$ internamente quattro setole piumate. Il ramo interno (fig. 12), esso pure bi-articolato, offre una serie di setole piumate crescenti in lunghezza dall' esterno all' interno $e$ in numero di sei sull'articolo terminale e di una sul breve e indistinto articolo basale.

Caratteristica è la presenza, sulla grande lamina basale del terzo paio di piedi natatori, di due cuscinetti assai sporgenti, di forma ovoide o sferica, guarniti di numerose piccole verruche, e di due bastoncini curvi, chitinici dal lato interno di esse, assai forti e sporgenti al di fuori a guisa di due molle. (') Presumo che le due protuberanze tondeggianti sieno organi d'adesione e invece i due bastoncini chitinici servano a tener sollevata la parte posteriore del cefalotorace dalla superficie dell'organo su cui vivono fissati questi parassiti, e ciò per permettere all'acqua di circolare e di aerificare le appendici natatorie del caligide.

Il quarto paio di piedi (fig. I3) uni - ramoso è costituito da quattro articoli; i1 primo basale è lunghissimo, gli altri tre che rappresentano il secondo segmento, presi insieme raggiungono appena la lunghezza del primo, e tutti tre i detti articoli sono armati da spinule, il primo e il secondo di una sola, l'ultimo di tre spinule decrescenti in lunghezza dall' interno all' esterno.

\section{Descrizione del maschio (Tav. XVI):}

Lunghezza $4 \frac{1}{2}-5 \mathrm{~mm}$. Come nella femmina lo scudo cefalico è quasi rotondo (fig. 81 essendo il suo diametro longitudinale presso a poco uguale a quello trasversale: però i suoi lati vanno restringendosi piuttosto verso l'avanti pur lasciando sussistere una lamina frontale abbastanza spaziosa, leggermente sinuosa nel mezzo e ricurva ai lati.

L'anelio toracico libero è molto più ristretto dello scudo cefalico e continua all' indietro coll'addome che si prolunga nel susseguente post-addome e che si conserva pure assai ristretto, solo l'addome presentasi di poco più largo verso

(1) Vedi l'identica organizzazionc nel maschio: Tav. XVI fig. II. 
1a sua parte terminale. Questi tre segmenti presi insieme presentano una lunghezza prosso a poco uguale a quelia dello scudo cefalico sopra menzionato.

Le antenne del primo paio sono bi-articolate. Il secondo articolo sottile molto più allungato del primo e più stretto.

Le antenne del secondo paio (fig. 9) hanno una disposizione pressochè orizzontale, il loro secondo articolo è grosso, striato sulla sua superficie e il terzo articolo unciniforme, ricurvo e con una spinula sul contorno interno.

Gli hamuli sono rivolti all' indietro e portano alla base una piccola spina rivolta verso l'interno.

Il rostro è di poco più lungo che largo e tozzo. Ai lati porta due palpi coll'estremità striata e con un piccolo dente sul contorno interno in modo da fare apparire l'estremità divisa in due rami di cui uno, l'interno, è allo stato rudimentale.

Il primo paio di piedi mascellari si presenta col secondo articolo, quello terminale, di forma allungata, sottile, più che il primo, e con una spinula sul margine interiore, verso la terza parte, vicino all' estremità libera; quest' ultima profondamente divisa in due rami affilati e appuntiti, di cui uno alquanto più lungo.

Il secondo paio di piedi mascellari (fig. Io) ha un largo e grosso pezzo basale che sul margine inferiore, in vicinanza del suo punto d' origine, mostra un dente vistoso. L'articolo unciniforme è bi-articolato e l'articolo basale sul lato interno, presso il punto della sua articolazione colla parte terminale, presenta una grossa setola a guisa di pungiglione.

La furcula sternalis è larga con due rami semplici, corti e divergenti.

Nel primo paio di piedi ogni piede è tri-articolato, l'articolo basale corto e più largo, con una setola e un'appendice sul contorno inferiore (quest'ultima è il rudimento del ramo natatorio interno), il secondo allungato quasi del doppio. L'ultimo articolo terminale breve porta tre spinule sull'estremità e quattro setole sul margine inferiore. Delle tre spinule quella esterna è un pochino più lunga che le altre due; quest' ultime ambedue coll' estremità bi-dentata. Delle quattrosetole sul margine inferiore, la prima quella d'angolo è una setola rigida e semplice, piu sottile e con lunghezza superiore delle spine; le altre tre setole sono piumate e assai sviluppate.

Il secondo paio, il terzo e il quarto come nella femmina. L'addome molto più stretto che nell' altro sesso, con qualche setola ai lati in vicinanza delle aperture genitali. Le due appendici caudali inserite sul post-addome (apparentemente uni-articolato), sono piccole e con quattro setole piumate. Anche qüi nel terzo paio di piedi, la lamina medesima più robusta, più spaziosa che di solito, presenta ai due lati due cuscinetti di forma ovale o sferica, guarniti di punte a guisa di tante verruche, e due pezzi chitinici ricurvi cilindrici ai loro lati, sporgenti fuori come due molle, organi che non ho mai osservato in nessuna altra specie di Caligizus (fig. II).

Là differenza più spiccata tra la forma maschile e quella femminile è data dalla struttura dell'anello genitale il quale, come si vedrà dalle figure, è molto più stretto nel maschio e diviso posteriormente in due lobi (se si guarda dal lato ventrale). Le appendici caudali, con quattro setole piumate, somigliano a quelle della femmina. 
Habitat. - Sulle branchie della Lichia amia Linn., Genova, 22 Aprile I89 I; nei seni frontali della stessa specie di pesce, Portoferraio, I9 Giugno I898. Questi ultimi esemplari furono raccolti dal D." Damiani.

Accettando la determinazione del compianto I. C. Thompson, nel mio primo lavoro (1898 (I) p. 208) avevo ritenuto questa specie affine col C. curtus sotto il quale nome ebbi allora a citarla in quel catalogo e più tardi anche in un'altra mia nota (1 899 (2) p. 4).

Caligns ligusticus n. sp., ठ․ Tav. XV, fig. I-8.

Caligus fissus Richiardi?, I880 (9) p. I48.

- - Carus, 188, (2) p. 359.

- - ? Brian, I898 (1) p. 209.

Descrizione del maschio:

Lunghezza $3 \mathrm{~mm}$. Lamina frontale grande con piccole ventose situate lateralmente, con due brevi antenne, senza incisione mediana. Cefalotorace (fig. I) grande quasi rotondo, più lungo della metà lunghezza del corpo, con due piccolissimi occhi posti dorsalmente sulla linea mediana e l'un l'altro attaccati. Ultimo segmento toracico piccolo e presso a poco sferico, congiunto coll' addome assai più grosso ma della stessa forma. Il post-addome molto più stretto, presenta due appendici guarnite ciascuna di tre lunghe setole piumate e di una quarta più breve (fig. 3).

Caratteri peculiari a questa forma si presentano nella forma rotondeggiante dello scudo cefalico, nel margine frontale spazioso e quasi senza curva, nelle lunule indistinte e piccolissime, tal chè non si vedono senza lente, e nella particolare costruzione del primo paio di piedi natatori e del quarto paio, che sono qui sotto descritti.

Le antenne anteriori (fig. 4) hanno l'articolo basale tozzo, tondeggiante e di poco più lungo che l'articolo terminale libero; l'uno e l'altro muniti di setole. Le antenne inferiori sono bi-articolate e potenti: il primo segmento, presso la sua base, è munito di una spina colla punta rivolta verso l'indietro.

Il primo paio di piedi mascellari sembra essere tri-articolato. E formato da un segmento basale breve soltanto separato per leggiero strozzamento dal seguente articolo più allungato, il quale a sua volta porta il terzo articolo sottilissimo, ripiegato sulla porzione basale e bifido all'estremità. Nel secondo paio di piedi mascellari ad un grosso e lungo segmento basale è fissato un uncino potente, ricurvo su sè stesso.

La furcula sternalis (fig. 5) presenta due rami semplici, ottusi alle loro estremità e alquanto divergenti.

Il primo paio di piedi natatori (fig. 6) si presenta, come di solito, con un solo ramo, costituito da tre articoli; il primo spesso e terminato superiormente da una setola; il secondo più sottile e più lungo, terminato esso pure da una piccola setola. L'ultimo, al suo termine, porta una grossa setola rigida e due piccolissime alla sua base, mentre il contorno inferiore presenta tre lunghissime setole piumate.

Nel secondo piede natatorio (fig. 7) il ramo interno è indistintamente tri-articolato anzi appare costituito da un articolo basale corto e da una grande lamina rettango- 
lare, allungata e foggiata in guisa da sembrare bi-articolata, e questa lamina porta nel suo contorno otto setole piumate crescenti in lunghezza dall' esterno all' interno. Anche l'articolo basale porta una setola piumata, verso l'interno. Il margine esteriore di questo ramo è ciliato. Il ramo esterno è invece distintamente tri-articolato e il primo articolo porta una setola da una parte e una spina lunghissima dall'altra. Parimente il secondo piuttosto corto, porta una setola e una breve spina uncinata, l'ultimo invece porta due piccole spine uncinate, una setola rigida e sei setole piumate che vanno crescendo esse pure dall' esterno verso l' interno.

Nulla di straordinario vi è da osservare nella forma del terzo paio di piedi natatori, foggiati secondo il tipo solito nei Caligus (fig. 8).

Il quarto paio di piedi è costituito da un articolo basale abbastanza lungo, e da una lamina piatta fogliacea e articolata con quello, la quale è formata da tre articoli; il primo e il secondo portano una setola rigida all'esterno e il terzo tre setöle rigide tutte assai lunghe e presso a poco di pari dimensioni.

I tre esemplari da me osservati sembrano maschi e tutti hanno $3 \mathrm{~mm}$. di lunghezza.

Habitat. - Sulle branchie di Box salpa Linn.: un esemplare proveniente da Genova e un altro da Portoferraio. Anche su di un Sargus Rondcletii Cuv. e Val. fu raccolto un altro esemplare di Caligus che ritengo debba riferirsi a questa specie, che mi fu inviato dal D.r Damiani dall' isola d'Elba.

Caligus productus DANA, $Q$.

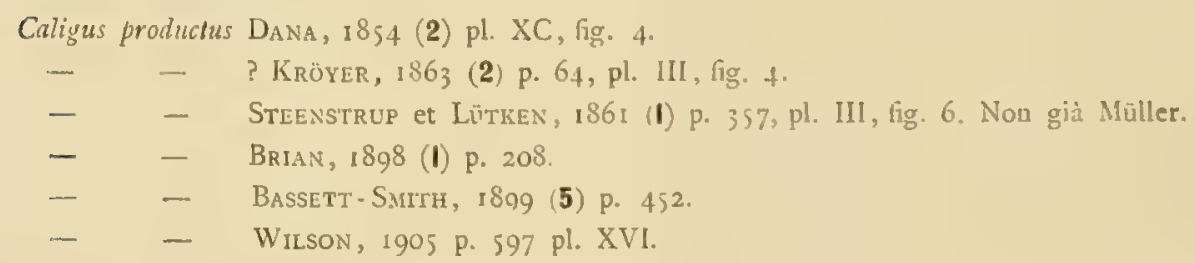

L'unico esemplare avuto in esame è una femmina lunga $4-4 \% \mathrm{~mm}$., assai malconcia. Osservata col microscopio dopo averla trattata colla potassa, ho potuto riconoscere nella forma delle appendici e nella costruzione generale di essa i caratteri principali propri della specie si da corrispondere alla figura che ne diedero lo Steenstrup e Lütken: solănto non mi apparve bene distinta la divisione in due articoli del post-addome, sebbene si presenti assai allungato. Ho notato il carattere saliente dato dalla mancanza di setole piumate nel primo paio di piedi natatori e quello offertoci dal quarto paio di piedi, muniti di cinque spine in lunghezza sensibilmente crescenti, leggermente curvate, l'ultima fra le altre poco più lunga. Le lunulae sono grosse e vistose; la furcula sternalis possiede due rami semplici, abbastanza allungati, dapprima alquanto divergenti e di poi colle estremità un poco convergenti.

Habitat. - La specie, che mi sembra rara nel Mediterraneo, non avendone finora rinvenuto che un esemplare solo, fu raccolta a Genova sulla Chrysophrys aurata Linn. E' stata indicata per le Indie occidentali sui pesci Coryphaena sp. e Balistes sp. 
Divisione II. Post-addome con due articoli.

Caligus Pelamydis Kröyer, $q$.

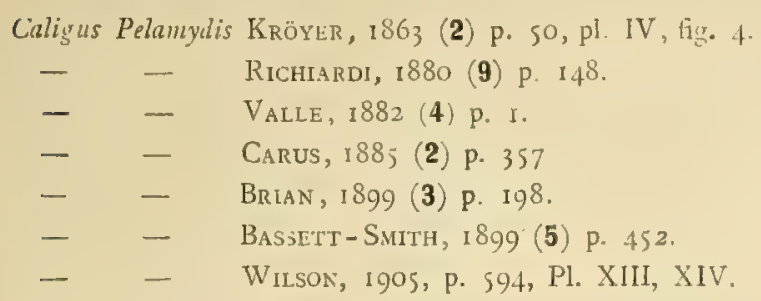

Distributio et habitat. - Questa specie è indicata da Kröyer come parassita della Pelamys sarda B1. ma in quale località non mi sembra risulti dal suo lavoro.

Carus tuttavia assegna a questa specie un' area di distribuzione estesa comprendendo anche 1'Atlantico, mentre Gerstaecker la cita soltanto pel Mediterraneo. Essa tanto da Richiardi, quanto da Valle è stata riscontrata sui lidi d'Italia, sia per l'Adriatico sia pel Mediterraneo. Il primo la ricorda come vivente sulla mucosa della cavità branchiale della Pelamys sarda Bl. e dello Scomber scomber Linn. Il secondo rinvenne parecchi esemplari di questa specie, il 28 Marzo I88I, sulla mucosa della cavità boccale e branchiale d'una Pelamys sarda B1.; parimente su di questo pesce ne furono raccolti esemplari dal D." Damiani a Portoferraio, il 2 Marzo I899, aderenti alla mucosa della cavità branchiale.

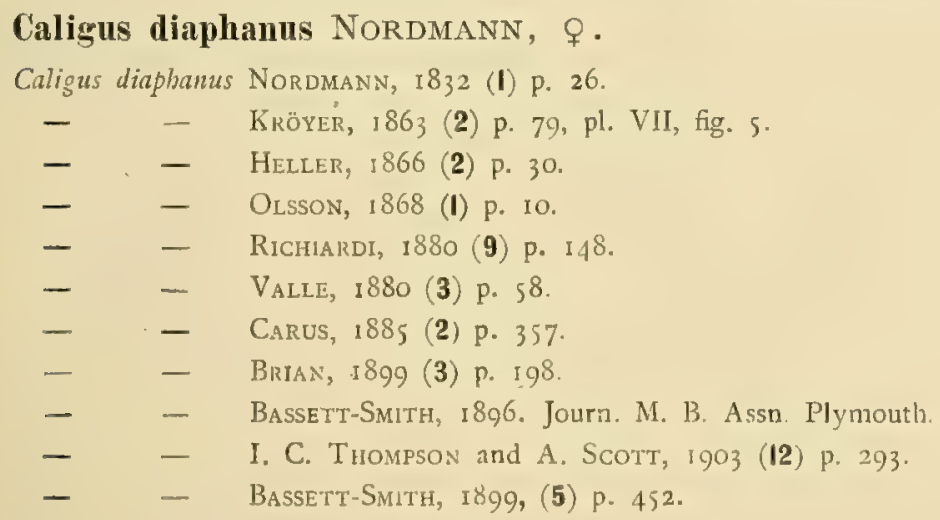

Distributio et habitat. - Questa specie non ha nulla a fare con quella omonima descritta da Baird e da M. Edwards. La sua presenza fu riscontrata da diversi naturalisti (Nordmann, Kröyer, Olsson) nell'Atlantico settentrionale sulle branchie di diverse specie di Trigla. Heller per primo la citò per i nostri mari ossia pell'Adriatico sulle branchie di Trigle lineata Linn. e T. corax Bp. Richiardi la rinvenne nel Mediterraneo su di un gran numero di ospiti: sulla mucosa della cavità branchiale di Pagellus mormyrus Cuv., P. erythrinus Cuv., Trigla cuculus Linn., T. corax Bp., T. milvus Lac, T. lineata Linn. e Platessa passer Bp. Valle per l'Adriatico non solo la riscontrò comune sulla pelle e sulle branchie della Trigla lineata Linn. e T. corax Bp. ma anche sulla T. aspera Viv. e T. Lyra Linn.

Io ebbi esemplari da Portoferraio raccolti colà dal D.r Damiani sulla mucosa della cavità branchiale di Trigla corax Bp., il 12 Maggio 1899 , sulle branchie di 
Pagellus mormyrus Cuv., 1'Ottobre rgor e sulle branchie di Pagellus acarne Cuv., il 5 Agosto rgor, e sugli archi branchiali di Trigla lineata Linn. il 9 Febbraio Igo3.

A Napoli raccolsi questa specie sulle branchie di Pagellus mormyrus Cuv. il 28 Agosto 1903 e sugli opercoli branchiali di Trigla corax Bp. il 27 Agosto rgo3. I. C. Thompson e A. Scott citano il Caligus diaphanus anche per la lontana località di Aripu (Ceylon), stata raccolta nella bocca e sulla pinna dorsale di un Therapon puta.

Caligus vexator Heller, $q$.

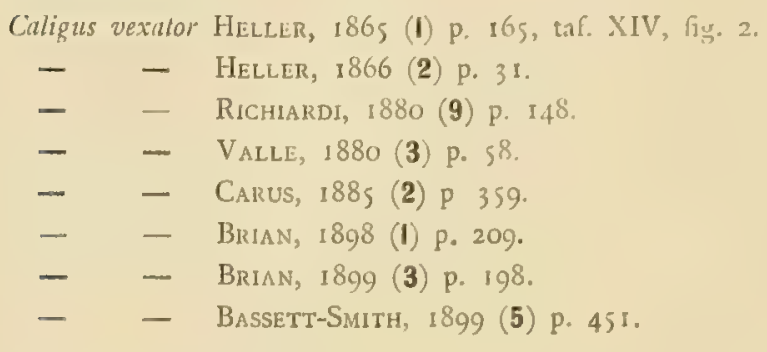

Distributio et habitat. - Heller descrisse per primo questa specie presa sulle branchie di Dentex vulgaris Cuv. e Val., nel Mediterraneo e nell'Adriatico.

Valle la trovò frequente sulle branchie di questo pesce nell'Adriatico. Richiardi pel Mediterraneo la cita, raccolta sulla mucosa della cavità branchiale di Dentex vulgaris Cuv. e Val., di D. gibhosus Rafn. e di Pagrus vulgaris Cuv. e Val.

Io riscontrai la sua presenza a Genova, proveniente dalle branchie di Dentex vulgaris Cuv. e Val. ( 2 Febbraio 1890 ) e parecchie volte ebbi esemplari da Portoferraio dal D. Damiani, una volta tolta dagli archi branchiali di Pagrus vulgaris Linn., il 2 Maggio I899, e altre volte dalle branchie di Dentex vulgaris Cuv. e Val. (Ottobre Igor e 24 Aprile 1903).

É specie esclusiva, per quanto mi consta, dei nostri mari.

Caligus Coryphaenae SteEnstrup et LüTKen, $¥$ e $\sigma$.

Caligus Coryphanae Steenstrup et Lütken, I86I (I) p. 360, pl. IV, fig. 7 .

Caligus bengoensis Scotr, 1895 (I) p. I30, pl. XIV, fig. I9.

Caligus Thymi? DANA, $1854(2)$.

Caligus scutatus? Edwards M., 1840 (2) p 453.

Caligus Coryphaenae Rıchiardi, I 880 (9) p. 148.

$\begin{array}{lll}- & - & \text { Valle, } 1880 \text { (3) p. } 58 . \\ - & - & \text { Carus, 1885 (2) p. } 358 . \\ - & - & \text { Brian, } 1899 \text { (2) p. } 4 . \\ \text { - } & - & \text { BasSeTt-Smith, 1899 (5) p. } 451 .\end{array}$

Distributio et habitat. - Questa specie studiata da. Dana, e descritta sotto il nome di Caligus Thynni (?) fu raccolta, secondo questo autore, nell'A tlantico ( $27^{\circ}$ lat. nord). Steenstrup e Lütken che descrissero con molta esattezza il Caligus Coryphaenae lo notano pure nell'Atlantico ( $30^{\circ}$ lat. nord) indicando come ospite una specie di Coryphaena. Sotto il nome di Caligus bengoensis, Scott lo cita per la fauna della Guinea, e sotto quello di C. scutatus (?), Milne Edwards ne registrò l'habitat nelle Indie orientali. È registrato inoltre per i lidi d'Italia dal Richiardi 
come parassita sulla mucosa della cavità branchiale della Coryphaena hippurus Linn., e per l'Adriatico da Valle come alquanto raro sulle branchie della Coryphaena pelagica Lac.

Io ne ebbi esemplari da Portoferraio inviatimi dal D.r Damiani che li trovò aderenti alle branchie di una Coryphaena hippurus Linn., il 23 Ottobre I898; e altri esemplari dalla stessa località raccolti pure sull'ospite ora riferito, il I 9 Settembre 1902.

\section{Caligus Pharanis Nordmann,}

Caligus Pharaonis NoRdMANN, 1832 (I) p. 28.

$$
\begin{aligned}
& -\quad-\quad \text { Edwards M., I840 (2) p. } 453 . \\
& -\quad-\quad \text { Gerstaecker (?) } \\
& -\quad \text { - Carus, I885 (2) p. } 557 .
\end{aligned}
$$

Habitat. - Fu trovato dapprima nel Mar Rosso sull' opercolo di un Chaetodon e fu indicato di poi pel Mediterraneo da Gerstaecker e da Carus.

\section{Caligus afïinis HeLLER,}

$$
\begin{aligned}
& \text { Caligus afinis HeLLER, I866 (2) p. } 30 . \\
& \text { - } \quad \text { - RICHIARDI, I880 (9) p. I48. } \\
& \text { - } \quad \text { - VALLE, I880 (3) p. } 57 . \\
& \text { - } \quad \text { - CARUS, I885 (2) p. } 357 .
\end{aligned}
$$

Habitat. - Heller riscontrò la presenza di questa specie nell'Adriatico sulle branchie di Umbrina cirrhosa Linn. Richiardi la ricorda pure pel Mediterraneo, da lui trovata sulla mucosa della cavità branchiale del pesce sopranominato. Su di questo ospite è citata anche da Valle per l'Adriatico, che la dice rara. Nessun autore ha dato di questà forma figura alcuna.

Specie non descritte:

\section{Caligus Trachini RICHIARDI,}

Caligus Trachini Richiardi, I 880 (9) p. I 48.

$$
\text { - - CARUS, I885 (2) p. } 359 .
$$

Habitat. - Sulla mucosa della cavità branchiale di Trachinus draco Linn. Mediterraneo.

\section{Caligus Trachuri RichIARDI,}

Caligus Trachuri Richiardi, 1880 (9) p. I48.

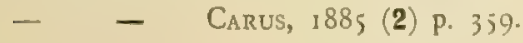

Irabitat. - Sulla mucosa della cavità branchiale di Trachurus trachurus Casteln. Mediterraneo.

\section{Caligus Sermani Richiardi,}

Caligus Serrani Richiardi, 1880 (9) p. 148.

$$
\text { - - Carus, 188; (2) p. } 359 .
$$

Habitat. - Sulla superficie della pelle di Serramus gigas Brünn. Mediterraneo. 
Caligus Lepidopi RICHIARDr,

Caligus Lepidopi Rıchiard, 1880 (9) p. 148.

- - Carus, 1885 (2) p. 359.

Habitat. - Sulla superficie della pelle di Lepidopus caudatus Euphr. Mediterraneo.

Caligus Petersii Richiardi,

Caligus Petersii Richiard, 1880 (9) p. I 48.

- - Carus, 1885 (2) p. 359.

Habitat. - Sulla mucosa delle arcate branchiali di Carcharias lamia Risso. Mediterraneo.

Gen. Lepeophtheirus NordmanN,

Divisione I. Post-addome con un articolo.

Lepeophtheirus 'Thompsoni BAIRD, $q$ e $d^{\circ}$

Caligus piscinus

? Caligus gracilis

Lepeophtheirus gracilis

? Caligus brancbialis

- $\quad-$

-

? Lepeophtheirus Rlombi

Leprophtheirus Thompsoni

- $\quad$ - BassetT-SMLTH, I899 (5) p. 455.

- $\quad$ - Thompson I. C. e ScotT A. 1903 (2) p. 294.

- $\quad$ - WIISON, 1905, p. 619. Pl. XVIII.

GUÉrin, 1840 pl. 35, fig. 2.

EDWARDS M. , 1840 (2) p. 456.

v. BENEDEN, 18 ; (3) p. 90, pl. 2 fig. 1-7.

RichIARDi, 1880 (9) p. 148.

CarUs, I885 (2) p. 359 .

BRIAN, 1898 (1) p. 210.

MALM (nsser.)

OLSOON, 1868 (I) P. I2

KRÖYER, 1863 (2) p. 118 pl. V, fig. 5.

o BRIAN, I 899 (3) p. I99.
Steenstrup et LütKen, i 861 (1) p. 362 pl. 11 , fig. 3.

Distributio et habitat. - Guérin descrisse per primo questo copepode col nome di Caligus piscinus e lo indicò nell' Oceano Atlantico sul "Merlan commun " (Morlangus vulgaris o Gadus merlangus). Van Beneden citava il suo Caligus gracilis, sinonimo del sopranominato, per il litorale belga sul corpo e nella cavità branchiale del Pleuronectes rhombus Linn. e del Rhombus maximus Linn., e asseriva di aver trovato in abbondanza questo parassita sul primo di questi due ospiti. Baird studiò esemplari alberganti sul Rhombres maximus Linn. e raccolti da Thompson in Marzo i 837 .

Richiardi distinse, come Van Beneden, gli esemplari di questa specie col nome di C. gracilis v. Bened. e li citò pel Mediterraneo, fissati sulla mucosa della cavità branchiale del Rhombus laevis Rond, e del $R$. maximus Cuv.

A Genova ho esaminato parecchi esemplari provenienti dalle branchie di un Rhombus maximus Cuv. Dal D.r Damiani ebbi un esemplare raccolto su di un tal ospite a Portoferraio, il 6 Marzo i 899. 
Malm, che nominò questa forma Caligus branchialis, la raccolse sulle branchie di Rhombus maximus Cuv. in Bahusia (Scandinavia). Olsson raccolse una sola femmina nella cavità branchiale di Rhombres laevis Rond. presso Grafverna, sulla spiaggia di Bahusia, nel mese di Settembre. Come parassita del Rhombus maximus Cuv., col nome di Lepeophtheirus Rhombi, è stata citata da Kröyer per il mare del Nord. Pure sotto questo nome io avevo determinato un esemplare maschio di questa specie, raccolto dal D. Damiani a Portoferraio sulla pelle di Rhombus laevis Rond. (1899 (3) p. I99).

Lepeophtheirus pectoralis NORDMANN, $q$.

Lernaea pecloralis MülleR, I777 (I) p. I25, Taf. XXXIII, fig. 7; Encyclop. Mèthod., Vers. Tab. LXXVIII, fig. I2.

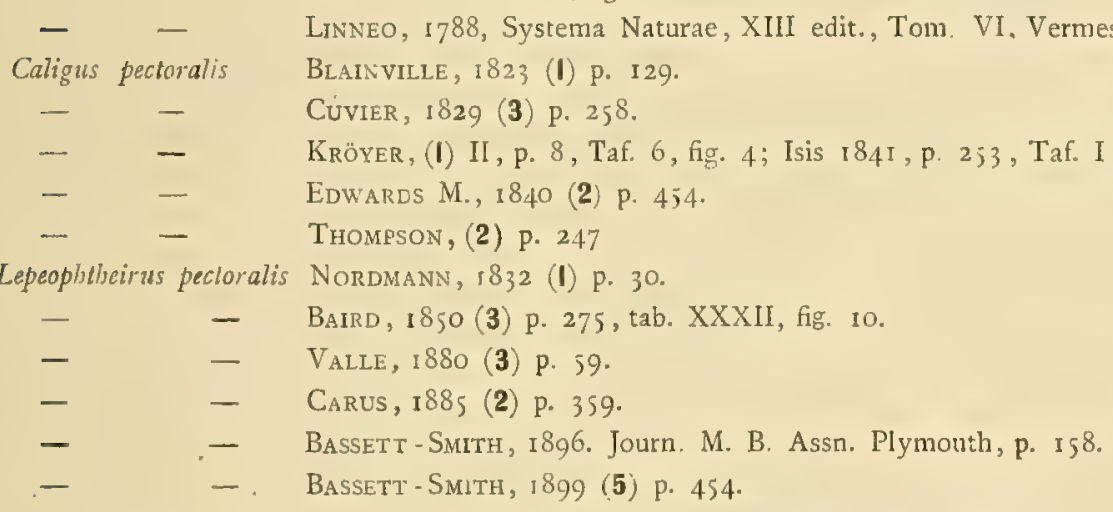

Distributio et habitat. - Nell' Atlantico e nel mare germanico.

Valle scrive che questa specie è comunissima sulla pelle e sulle branchie della Platessa passer Linn. e del Rhombus maximus Linn. (Psetta maxima Sw.) nell'Adriatico.

Lepeophtheirus Nordmanni M. EDWARd, $q$ e $\sigma$. Tav. I, fig. I.

Caligus Nordmanni

EDWARDS M., 1840 (2) p. 455.

Edwards M., I849, Atlas du Régne Animal de Cuvier, Crust., tab. IXXVII, fig. 1 , feni.

Thompson, (2) p. 248.

Lepeoplstheirus Nordmani BaIRD I850 (3) p. 275 Tab. XXX11I, fig. I fem.

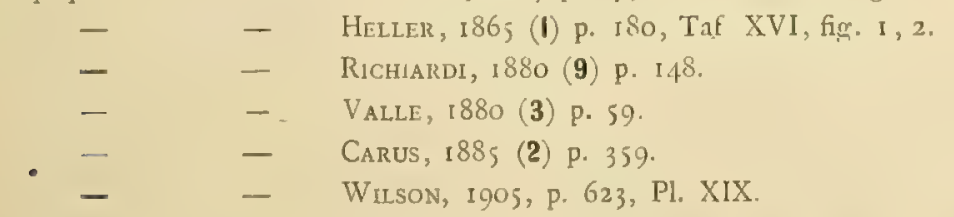

Lepeophlheirus Hippoglossi Brian, 1898, (1) p. 210 , tav. I, fig. 4 tav. II, fig. 13.

$$
\text { - - Brian, I809, (3) p. I99. }
$$

Distributio et habitat. - Sotto il nome di Caligus Nordmanni, Milne Edwards lo descrisse nell' « Hist. Nat. des Crustacés » e dice che fu trovato a Nizza sulla pelle di una Mola. Baird ne dà la descrizione nel suo «Nat. Hist. of the British Entom. » da esemplari presi pure sull' Orthagoriscus mola Linn., lungo la costa d'Irlanda in provincia d'Antrim, nel Settembre 1848. Come parassita dello stesso pesce è citato da Heller pel Mediterraneo; sul quale ospite per questo mare lo indica 
pure Richiardi, come parassita sulla mucosa della cavità boccale. Valle riscontrò invece la sua presenza sulle branchie di detto pesce e ne ebbe un solo esemplare dall'Adriatico. Io ne esaminai molti esemplari raccolti sopra di un Orthagoriscus mola Linn. catturato a Pegli, il 2 Giugno $189 \mathrm{I}$, ed altri esemplari presi da un altro simile ospite pescato il 26 Maggio rgor a Genova. Nel mio catalogo dei cop. par. della Liguria, risultando essi determinati impropriamente per Lepeophtheirus Hippoglossi Kr., vi si faccia la correzione opportuna, sostituendo il nome di L. Nordmanni a quello; e lo stesso sia detto a riguardo di un esemplare raccolto dal D. Damiani a Portoferraio sull' Orthagoriscus mola, il 9 Marzo 1899, che avevo registrato come $L$. Hippoglossi $\mathrm{Kr}$. in un'altra mia nota (I899).

Nell' estate Igo3 rinvenni pure a Napoli parechi esemplari di questa specie interessante, sulla pelle di un pesce simile.

Divisione II. Post-addome con due articoli.

Lepeophtheirus Grohmanui KRöYER, ?.

Lepeophtheirns Grohmanni KRÖYER, I863 (2) p. 108, pl. V. fig. 3.

- $\quad$ - Carus, 1895 (2) p. 359.

- $\quad$ - Bassett-SMith, I899 (5) p. 456.

Habitat. - Pleuronectes (Arnoglossus) Grohmanni Heck. Mediterraneo: Sicilia (Mus. Vindob., Havn. Kröyer).

Lepeophtheirus Trygonis VALLE,

Lepeophtheirus Trygonis VALLE, I 882 (4) p. I.

$$
\text { - C Carus, i88; (2) p. } 360 .
$$

Specie non descritta. Valle trovò due soli esemplari di questo nuovo caligide il 21 Maggio I88I, sulla mucosa della cavità branchiale d'un colossale Trygon pastinaca Linn.

\section{Gen. Diphyllogaster BRIAN,}

Diphyllogaster Thompsoni BRIAN, $q$.

Dipbyllogaster Thompsoni Brian, 1899 (4) p. 53, tav. 3.

Habitat. - Sulle branchie di Dicerobatis Giomae Gunt. Mare ligustico (Genova).

\section{Gen. Lütkenia Claus.}

Lütkenia Asterodermi Claus, $\rho$ e $\sigma$.

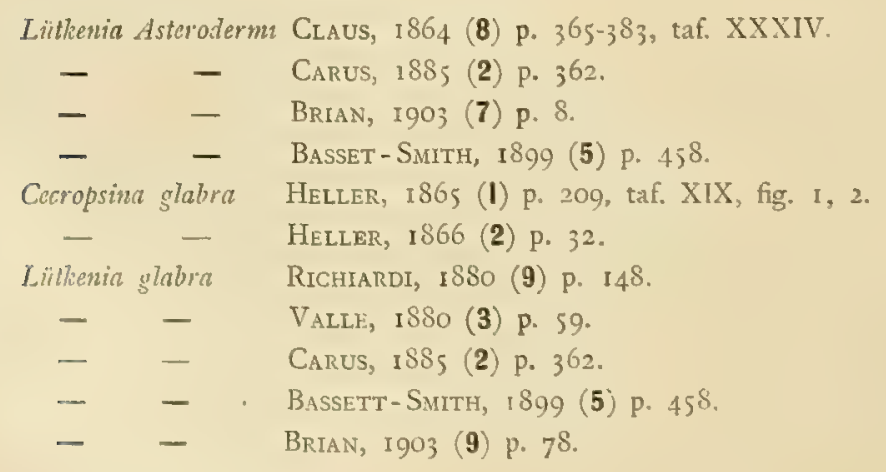


Habitat. - La Iütkenia Asterodermi Claus è, secondo me, sinonima della Liutkenia glabra. Questa fu descritta sotto il nome di Cecropsina glabra da Heller nel 1865, da esemplari raccolti, non si sa su quale pesce, nel mare Adriatico. Heller citava di nuovo questa specie un anno dopo nel suo elenco di crostacei del mare Adriatico, e neppure allora nominava l'ospite. Col nome di Lütkenia glabra, questa stessa specie, comparve nel catalogo del Richiardi nel r880, e questa .volta era stata raccolta sulla inucosa della cavità branchiale del Luvarus imperialis Raf. Contemporaneamente Valle presentava, sotto lo stesso nome, il parassita del Luvarus e lo diceva comunissimo sulle branchie di detto ospite. La sostituzione del nome Lütkenia al genere Cecropsina, era necessaria, perchè il Claus nel I864, un anno prima che Heller facesse conoscere la sua forma, aveva già descritto la Lütkenia Asterodermi, specie che per.i caratteri generici doveva identificarsi. colla Cecropsina. La forma descritta da Claus era stata raccolta a Messina sulle branchie di un Asterodermus coryphaenoides, pesce che sotto questo nome corrisponde ad uno stadio giovanile dell' anzidetto Luvarus. Notando per tal modo che le due specie di Lütkenia or dette sono come parassite ambedue di uno stesso ospite, sebbene questo si presenti in diverso stadio di sviluppo, mi è sorto il dubbio che le due specie possano essere identiche fra loro, e avendo confrontate le descrizioni e le figure di una specie e dell' altra secondo Claus ed Heller, e dopo esame dei relativi esemplari raccolti a Genova e a Portoferraio, mi sono accertato che sussiste l'identità fra di esse.

Questa è una forma propria soltanto dei nostri mari. Sotto il nome di Lütkenia Asterodermi risulta già citata da me per la Liguria, raccolta sulla pelle dell'Asterodermus elegans Risso, a Monterosso, il is Novembre Igo2.

Col nome corrispondente di Lütkenia glabra l'ho pure citata per l'isola d'Elba colà raccoltavi dal D. Damiani sulle branchie di Luvarus imperialis Raf, il 26 Luglio 1901. Altri esemplari furono trovati sulle branchie di questo stesso pesce il 23 Novembre I 888 e nel 1892 a Genova.

\section{Lütkenia integra RICHIARDI,}

Lütkeria integra Richiard, I880 (9) p. 148.

- $\quad$ - Carus, 1885 (2) p. 362.

Specie non descritta. Richiardi ascrive ad essa come ospiti: Galeus canis Linn. e Mustelus equestris Bp. (sulle branchie). Mediterraneo.

Non conosco questa specie. Avevo creduto di dovere riferire a lei, sotto questo nome, una forma di dubbia determinazione (vedi mia op. cit. I898 (1) p. 2 I I, tav. II, fig. 7), che oggi ho riconosciuto per Nesippus orientalis Heller.

\section{Gen. Nesippus Heller,}

\section{Nesippus orientalis HeLLER, 오}

Nesippus orientalis HELLER, 1865 (1) p. 194, pl. XVIII fig. 2.

- - B BSSETT - SMith, I899 (5) p. 459.

Lütkenia integra BRIAN, 1898 (I) P. $2 \mathrm{Ir}$, tav. II fig. 7 .

Habitat. - Secondo Heller questa specie fu trovata sulle branchie di Prionodon menisorrah a Java. A Napoli ne raccolsi pochi esemplari sulle branchie di Alopias 
vulpes Linn., 1903. A Genova fu trovato un esemplare che ritenni dapprima come spettante alla Lütkenia integra n. sp. di Richiardi, ma che più tardi riconobbi appartenere al Nesippus orientalis. Questo esemplare era stato raccolto sulla mucosa della cavità boccale del Mustelus laevis Risso, nel Settembre I89o.

\section{Gen. Nogagus LEACH,}

Nogagus caelebs HeLLER, o7.

Nogagus caelebs Heller, 1865 (I) p. 208, pl. XX, fir. 4 .

- - C Carus, 1885 (2) p. 360

-. - - BAssetT - SMith, 1899 (5) p. 460.

Habitat. - Questa specie è stata citata dall'Heller pel Mediterraneo, ma egli non indicò su qual ospite.

Nogagus Latreillii LEACH, $\sigma$.

Nogagus Latreillii LEACH, 1819 (2) p 536.

- - - Edwards M., 1840 (2) p. 459.

- - - Steenstrup et Lütken, i86r (I) p. 384, pl. IX, fig. 18.

- $\quad$ - Brian, 1905 (10) p. 6.

Habitat. - Ho rinvenuto un esemplare di questa specie conservato entro un tubetto nel Museo Zoologico di Napoli, stato raccolto dal Costa in quel golfo non si sa su quale pesce. Tale forma nell'Atlantico era stata trovata sopra di una specie di Carcharias.

\section{Gen. Demoleus HELLER,}

Demoleus paradoxus Heller, $q$ e $\sigma^{7}$.

Caligus paradoxus Oтro, Nov. Acta Acad. Caes. Leop., t. XIV, p. 352 (tav. XXII, igg. 5)

- - NordmanN, 1832 (I) p. 32.

Nogagus produclus GERSTAECKER, I 853 (I) s. 54 ; fo I- 10.

Demoleus paradoxus Heller, I86; (I) p. I99, taf. XIX, fig. 3 .

Nogagus grandis? of Steenstrup et LüTken, I861 (1) p. 386, tab. X, fig. I9.

Demoleus paradoxus Carus, i885 (2) p. 36 r.

- - BissetT-SMith, 1899 (5) p. 46 t (Heller).

Habitat. - Questa specie fu raccolta nel Mediterraneo su di un pesce-cane

\section{Gen. Trebius KRöYER,}

Trebius caudatus KRöYER, + e $\sigma$.

Trebius caudalus KRÖYER, (I) II 30.t. I, fig. 4.

- - Edwards M., I840 (2) pag. 458.

- - Thompson, 1847 (2) p. 248 ,

- - BAIRD, 1850 (3) p. 280 tab. XXXIII, fig. 3, 4.

Trebins spinifrons? Edwards M., I840 (2) p. 458, pl. XXXVIII, fig. I.

Trebius caudatus KRÖYER, I863 (2) pag. I49, tab. X, fig. I.

-_ - O OLSSON, 1868 (I) p. 14

- - V Valle, i880 (3) p. 60.

- - Carus, i885 (2) p. 360

- - Brian, IgO2 (5) P. 33.

- - Bassett-Smith irSg9 (5) p. 46 I. 
Distributio et habitut. - Tale specie fu dapprima rinvenuta nel mare germanico (Atlantico del Nord). Kröyer le ascrive come ospiti: Galeus vulgaris Flem. e Raja batis Linn. Olsson ricorda oltre a quest' ultimo pesce anche la Raja fullonica Risso e la cita per la fauna scandinava. Baird descrisse esemplari tolti da una Raja batis Linn. catturata nella località di Belfast Bay nel Settembre del I838. Milne Edwards non fornisce indicazioni precise e la dice specie trovata su di uno squalo nel mare del Nord: e lo stesso ripete a riguardo del Trebins spinifrons che ritengo sia sinonimo.

Valle riscontrò la presenza di questa specie nell'Adriatico vivente nel cavo della bocca dell'Acanthias vulgaris Risso. Un esemplare è stato da me indicato per Portoferraio, dove il D. Damiani lo rinvenne aderente all' atrio degli spiracoli nasali di Raja macrorynchus Raf.? Un altro esemplare (femmina) 'l' ho raccolto a Napoli sulle branchie di un Acanthias vulgaris Risso, il 2 I Agosto I 903.

\section{Gen. Elytrophora Gerstaecker,}

Elytrophora brachyptera GERSTAECKER, 우 e $\sigma$.Tav. I, fig. 2-3.

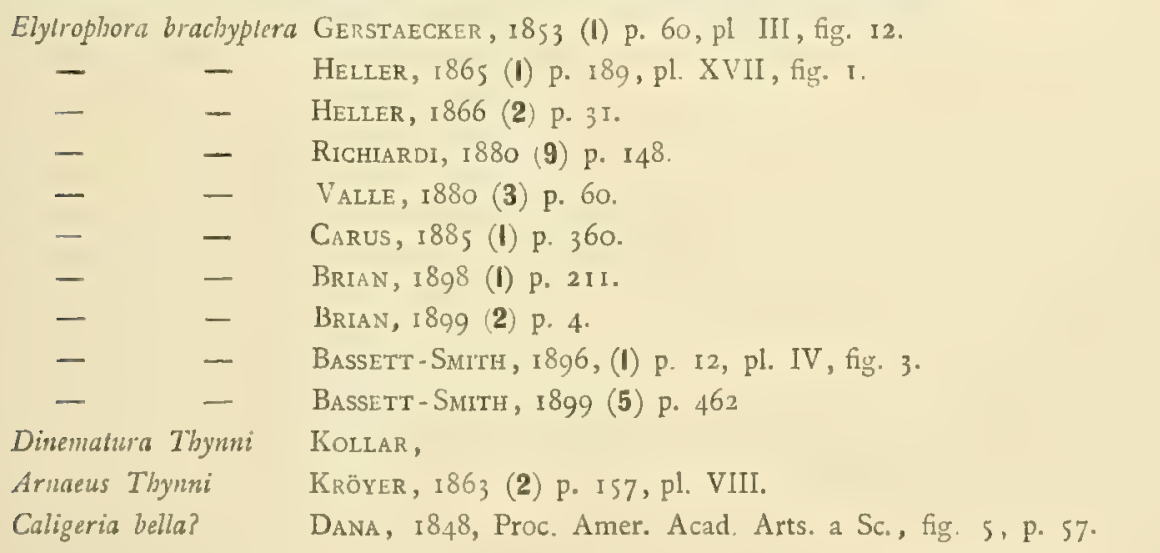

Distributio et habitat. - Gerstaecker cita questa specie come propria del Mediterraneo ed anche Heller riscontrò la sua presenza nei nostri mari. Essa vive nella cavità orale del tonno e dal Richiardi è indicata pure sulla mucosa della cavità branchiale di questo pesce. 亡̇ ricordata parimente da Valle per 1'Adriatico. Bassett - Smith cita anche questo caligide in Inghilterra nelle acque di Plymouth, tolto dalle branchie di un grosso Thynnus thynnus White. Kröyer ne illustra esemplari sotto il nome di Arnaeus Thynni che erano stati raccolti in Sicilia sopra di un tonno e che erano stati catalogati nel Museo di Vienna col nome di Dinematura Thynni da Kollar. L'affinità di questi esemplari con quelli descritti da Gerstaecker e da Heller è evidente.

Notai anch'io la presenza di questa specie a Genova, avendo osservato numerosissimi esemplari raccolti dalle branchie del Thynnus thynmus White. Altri esemplari mi pervennero da Portoferraio e altri ancora rinvenni a Napoli. 


\section{Gen. Dinematura LATREILLE,}

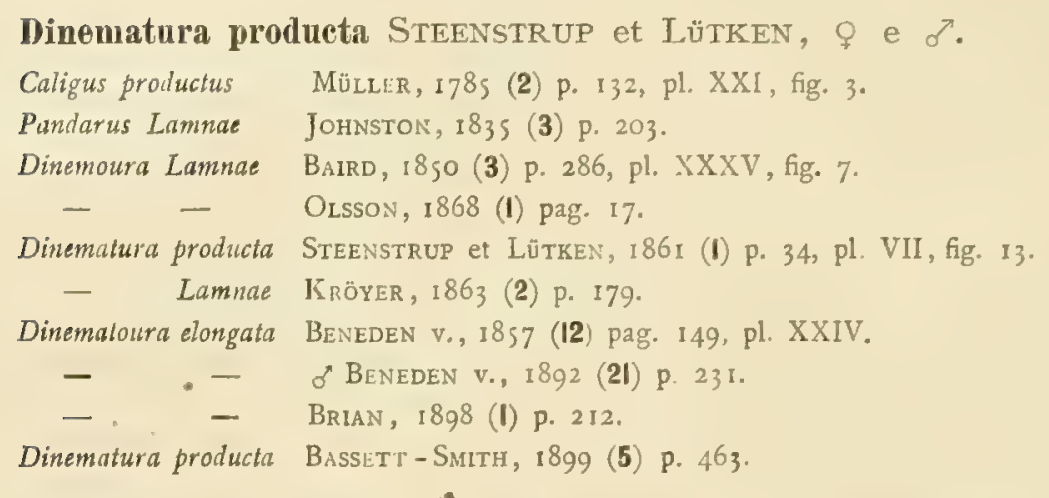

Distributio et habitat. - Van Beneden studiò esemplari di questa specie raccolti sulla pelle di Scymnus glacialis (Sc. microcephalus Bloch) nel mare del Nord dal prof. Eschricht. Quell' autore scrive che qualche parte del corpo d'uno Scymnus ne era letteralmente ricoperta, al punto tale da assumere un aspetto scaglioso. Steenstrup e Lütken dicono che questa specie vive parassita sulla Lamna cornubica Linn. (Atlantico). Il Dr. Johnston illustrò siffatto caligide raccolto nel Settembre 1834 su di detto pesce in Berwick Bay, (Atlantico). Gerstaecker cita come ospite di esso anche uno Squalus sp. Dall' Hope è citato come parassita della Lamna comubica Linn. per la località di Nizza. Dopo di lui, per quanto mi consta, nessun altro lo indicò pel Mediterraneo. Io ebbi la fortuna di osservarne esemplari, nel Museo Zoologico di Genova, stati raccolti sopra un individuo giovane $\checkmark$ di Selache maxima Günner, catturato a Camogli il 26 Agosto I888. Un altro esemplare fu raccolto dal D. Damiani e inviatomi da Portoferraio. Anch' esso era stato preso dalla cute di una Selache maxima colà catturata nel I903.

Il maschio delle Dinemature, che era ancora ignoto sino a qualche tempo fa, fu scoperto e descritto, precisamente per questa specie, da Van Beneden nel 1892. Lo trovò in mezzo ad un gran numero di femmine, sulla pelle d'una Lamna cornubica Linn. pescata sulle coste di Bretagna.

Dinematura latifolia STEENSTRup et LüTKEN, $q$.

Dinemalura latifolia SteEnstrup et LüTKEN, I86 I (I) p. 38, pl. VIII, fig. 16

$$
\begin{aligned}
& \text { - } \quad \text { - Hellek, i865 (I) p. Ig8. } \\
& \text { - - - Richiardi, I880 (9) p. I48. } \\
& \text { - - Valle, i880 (3) p. } 60 . \\
& \text { - } \quad \text { - Carus; i885 (2) p. } 360 . \\
& \text { - - BRIAN, I898 (1) p. } 212 . \\
& \text { - - BRIAN, } 5899 \text { (3) p. } 200 . \\
& \text { - - BasserT-Silth, I899 (5) p. } 463 .
\end{aligned}
$$

Distributio et habitat. - Ospite di questa specie, secondo Steenstrup e Lütken; è l'Oxyrhina glauca Müll. e Henle. Essi la citarono pell'Atlantico boreale.

Richiardi la ricorda pel mare d'Italia, raccolta sul Prionodon glaucus Linn.; Valle per 1'Adriatico sul Carcharodon Rondeletii Müll. e Henle. 
Ho esaminati varii esemplari presi a Carloforte dal Prof. C. Parona sull'Alopias vulpes Linn. e altri trovati sull'Oxyrhina Spallanzanii Raf. a Genova. A Portoferraio il D.r Damiani ne raccolse un esemplare sulla pelle dell'ultimo ospite ora indicato, il 2 I Giugno I899; e due altri il I3 Luglio I9or.

Heller citava tale specie pel Mediterraneo trovata su di un pesce-cane.

\section{Gen. Philorthagoriscus Horst,}

Philorthagoriscus serratus (KR.) Horst, f e ơ. Tav. I, fig. 4, Tav. XVI, fig. I-7.

Dinematura serrata KRÖYER,

Dinemoura serrata KRöYER, 1863 (2) p. 176 , pl. VIII, fig. 4.

Philorthagoriscus serratus HoRsT, I 897 (2) p. I37-144.

Distributio et habitat. - Kröyer non ci diede nessuna notizia circa l'habitat di questo parassita.

Nell' estate 1903 raccolsi molti esemplari dei due sessi di questa specie a Napoli sull'Orthagoriscus mola Linn. Esaminati freschi, quasi ancora viventi, avevano un colore rosa-pallido. Il loro corpo semi-trasparente lasciava scorgere gli organi interni e sopratutto gli ovari di colore giallo-rosso mattone chiaro. Di colore giallo un po' più scuro si presentavano le parti chitiniche come ad es. le estremità uncinate delle seconde antenne e dei piedi mascellari del secondo paio.

Un esemplare osservato la prima volta in Genova fu da me ascritto fra i Laemargus muricatus coi quali somiglia salvochè per le dimensioni, e perciò l' ho citato con questo nome nel mio catalogo (1). Anche Hesse nel descrivere questa ultima specie diede il disegno di forme in tutto somiglianti alla Dinematura serrata e che egli ritenne per forme giovanili di Laemargus muricatus.

Come si vede, Horst compose il nome del genere togliendolo dal nome del l'ospite abituale di questo caligide, che è l'Orthagoriscus mola Linn., il pesce anzidetto.

\section{Gen. Echthrogaleus STEenstrup et LüTKEN,}

Echthrogaleus coleoptratus STEENSTRup et LüTKEN, Q. Tav. II, fig. 2.

Dinemoura coleoptratus GuérIN, 1840 , pl. XXXV, fig. 6 .

Pandarus alalus Johnston, 1836 (3) p. 202.

Dinemoura alata EDWARDS M. (2) p. 464.

Nogagus? $d$.

Echthrogaleus coleoptratus STEenstrup et LüTKEN, 186i (I) p. 380 , pl. VIII, fig. IS.

$$
\begin{aligned}
& \text { - - Otsson, } 1868 \text { (1) p. } 20 \\
& \text { - } \quad \text { - BRIAN, } 1899 \text { (2) p. } 4 . \\
& \text { - } \quad \text { - Brian, } 1902 \text { (5) p. } 36 . \\
& \text { - } \quad \text { - BassetT-SMith, } 1899 \text { (5) p. } 464 .
\end{aligned}
$$

Distributio et habitat. - Osservai come nuova pel Mediterraneo questa specie per la prima volta a Genova, raccolta dal Sig. Borgioli sul Centrophorus granulosus Bloch, pescato nel mare ligure, il 6 Aprile I898, ed in seguito altri esem- 
plari furono presi dal D.r Damiani sotto l'ascella della pinna dorsale del Charcharodon Rondeletii Müll. e Henle, il 5 Giugno r898 a Portoferraio.

Gli esemplari descritti da Steenstrup e Lütken furono trovati nell'Atlantico dal capitano Hygom a $38^{\circ}$ lat. n.

Baird che ha descritto questa specie sotto il nome di Dinemoura alata, la dice raccolta dal D." Johnston nel 1834 su di una Lamna cornubica Linn. pescata nell'Atlantico, nella baia di Berwick. Da Milne Edwards è citata pei mari dell' India dove fu rinvenuta sopra di un pesce-cane. A detta di Baird sembra essere parassita anche di altri pesci.

\section{Gen. Oecrops LEACH,}

Cecrops Latreillii LEACH, e $\sigma$. Tav. II, fig. 3,4.

Cecrops Latreillii LEACH, 1816 (I) pl. XX, fig. I-S.

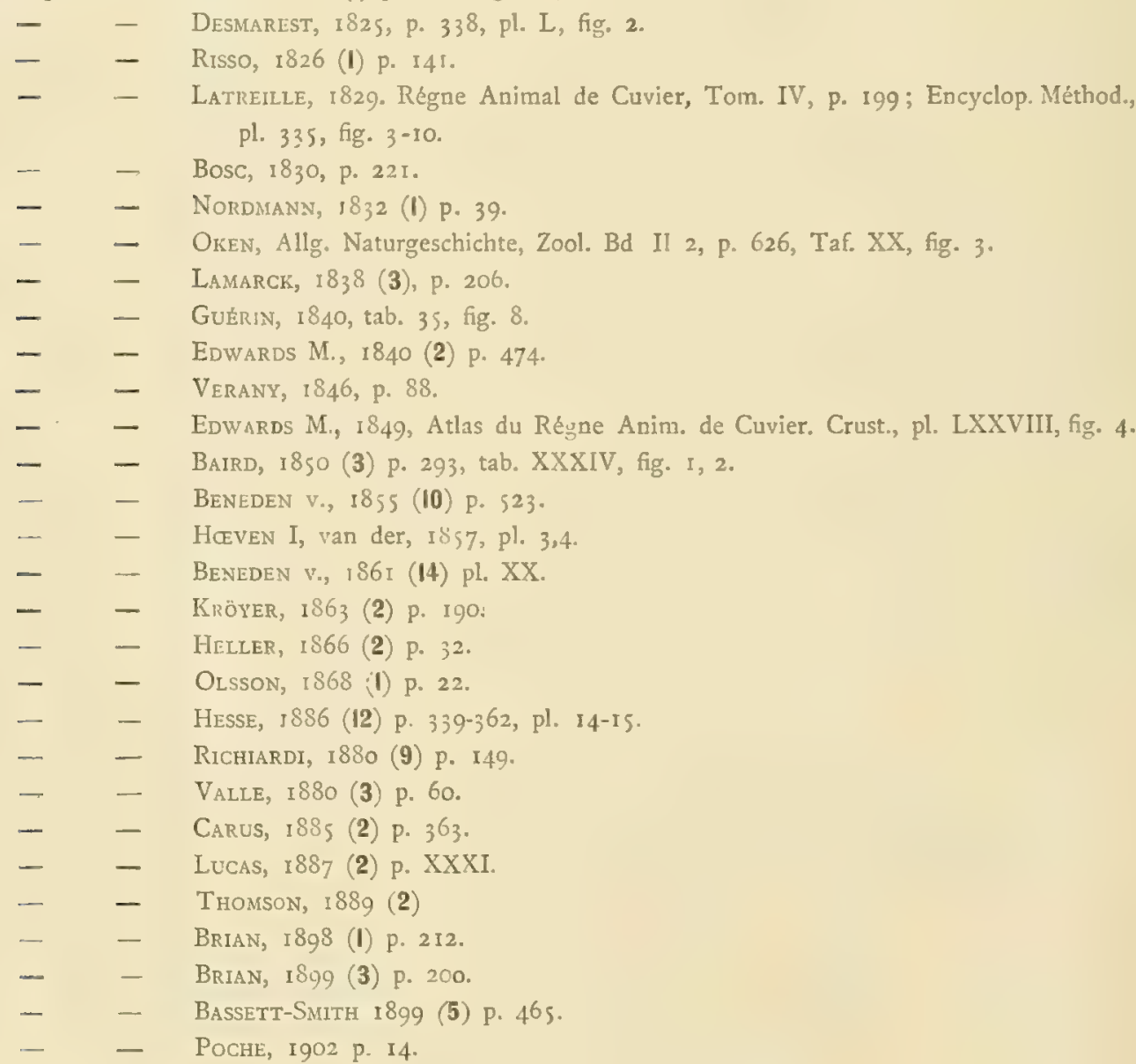

Distributio et habitat. - La prima volta fu trovata da Lamartinière presso le coste della California sulle branchie di un Diodon; in seguito fu raccolta moltissime volte nell'Atlantico settentrionale. Lucas riporta come ospiti di questa specie il Thynnus thynnus White, il Pleuronectes maximus Linn. il P. rhombus Linn. e l'Orthagoriscus mola Linn.; ma è sopratutto sulle branchie di quest'ultimo che è 
stata rinvenuta sovente nell"oceano Atlantico. Anche nei mari d'Italia è specie discretamente comune.

Nel catalogo dei crostacei liguri del Verany (1846) noi troviamo indicato i1 Cecrops Latreillii Leach senza indicazione d'ospite. Risso, 1826 , nel suo «Hist. Nat. Eur. Mér. » p. 14I, lo indica per la località di Nizza e a torto come nutrimento dell'Orthagoriscus mola Linn. ritenendo questi caligidi come animali pelagici.

Una discreta quantità di tali copepodi fu raccolta a Genova nel Museo di Zoologia della R. Università sopra una Orthagoriscus mola Linn., il 2 Maggio $189 \mathrm{r}$.

A Portoferraio la specie fu trovata sulle branchie del pesce ora riferito da D. Damiani il 9 Marzo I899. Per la località di Napoli questa forma fu inđicata dal Costa e dall' Hope, e colà anch' io (nell' estate del rgo3), ebbi a raccoglierne alcuni esemplari su di un pesce-luna. Heller e Valle la citano per l'Adriatico raccolta sul Thynnus thynnus White, e Richiardi pel Mediterraneo vivente sia sulle branchie di quest' ultimo ospite, sia su quelle dell' Orthagoriscus mola Linn.

\section{Gen. Phyllophora M. EDwARDS,}

\section{Phyllophora crassa Richiard,}

Phyllophora crassa RichiARDI, I880 (9) p. I 48.

$$
\text { - Carus, i885 (2) p. } 36 \text { i. }
$$

Habitat. - Specie non descritta. Richiardi la rinvenne aderente alle branchie di Prionodon glaucus Linn. Mediterraneo.

\section{Gen. Pandarus LEACH,}

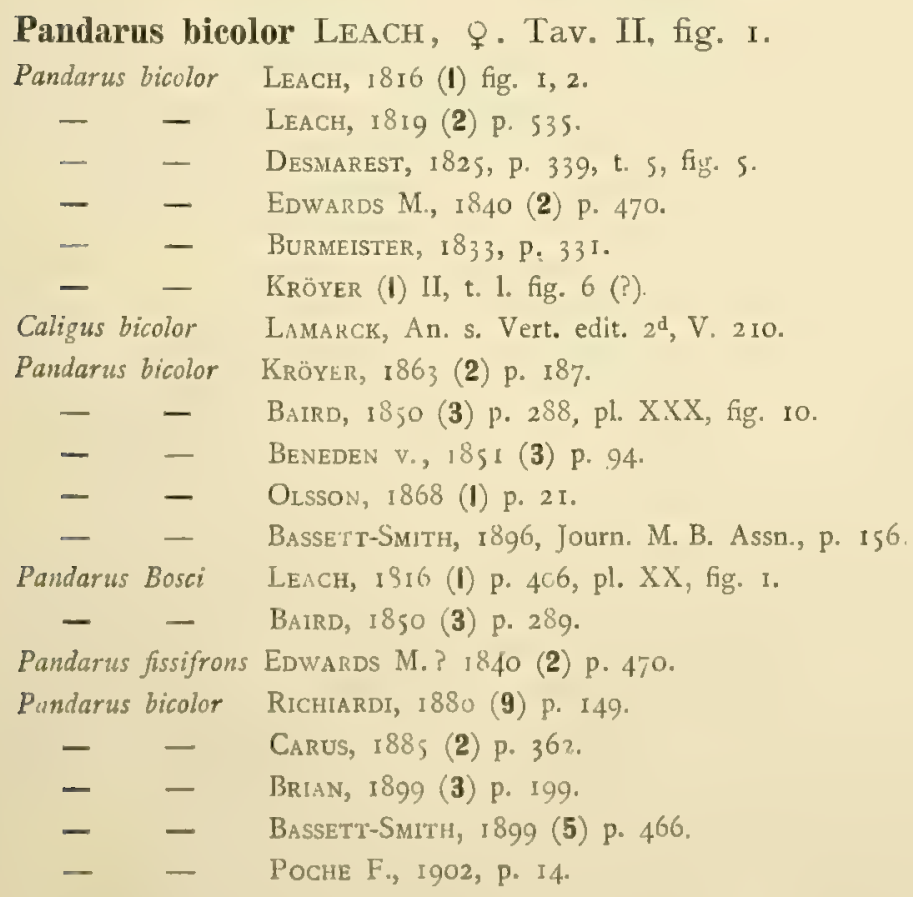

Distributio et habitat. -- Questa specie è stata raccolta da principio più volte nelle acque dell'Atlantico settentrionale e orientale. Leach la rinvenne sul Gaieus 
canis Linn. a Torcross nel Devonshire dove pure ha riscontrato la presenza della varietà $P$. Boscii, da lui trovata sullo Squalus mustelus Linn. Baird la indica come parassita di un Prionodon glaucus Cuv, catturato a poche miglia dal porto di Falmouth nel I849, in Inghilterra. Bassett-Smith dà per ospite di questa specie anche lo Scyllium catulus Cuv, e Gerstaecker l'Acanthias vulgaris Bp. (sulle branchie).

Per quanto riguarda la fauna italiana un esemplare fu raccolto dal D.' Damiani a Portoferraio sulle pinne pettorali di Galeus canis Linn., il 7 Luglio rgo3. Per 1'Italia tale specie è ancora ricordata da Richiardi che la rinvenne sulla mucosa della bocca di Prionodon glaucus Linn.

\section{Pandarus lugubris Heller, $q$.}

Pandarus lugubris HeLleR, 1865 (1) p. 205, pl. XX, fig. I.

$$
\begin{aligned}
& \text { - } \quad \text { - Carus, i885 (2) p. } 362 . \\
& \text { - - BassetT-SMith, I899 (5) p. } 467 . \\
& \text { - - Brian, } 1902 \text { (5) p. } 36 .
\end{aligned}
$$

Habitat. - Fu rinvenuto da Heller sopra di una specie di squalo nel Mediterraneo. A Genova ne fu trovato un solo esemplare lungo $6 \% \mathrm{~mm}$. sulle branchie dell' Oxyrhina Spallanzanii Raf. (Luglio I89o).

\section{Gen. Orthagoriscicola Poche,}

\section{Orthagoriscicola muricatus KRÖYER, + e $\sigma^{7}$.}

Laemargus muricarus KRöYeR, 1837-38 (I) I, p. 487, tab. S, fig. A, B, C, D; IsIs, 1841, p. I04, Taf. II, 5, fig. A-D.

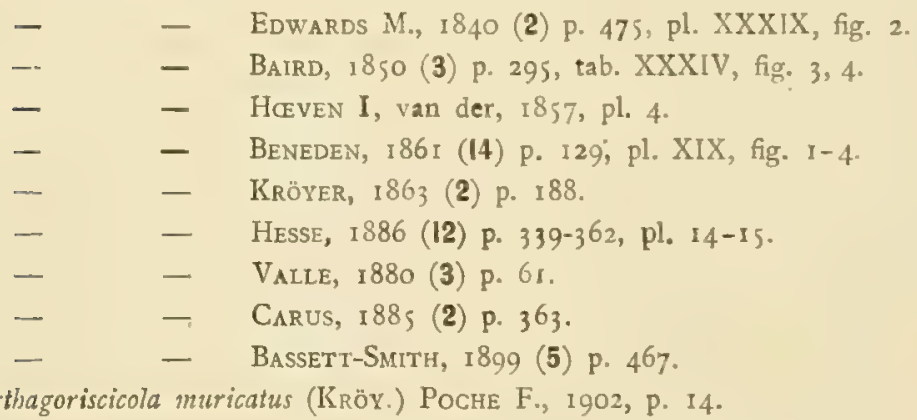

Distributio et habitat. - Non ho riscontrato la presenza di questa specie nel mare ligure ('), seppure sia stata citata per l'Adriatico da Valle al quale il D: Ed. Gräffe, Ispettore dell'I. R. Stazione Zoologica di Trieste, comunicò di averne trovato in quel mare, tre esemplari sopra un Orthagoriscus mola Linn.

Da Hesse per contro sono citati esemplari per l'Atlantico dove, egli dice, vivono in gran numero sulle branchie di detto pesce e sono piuttosto comuni. Ad esempio ne raccolse copiosamente il I5 Novembre 1879 a Brest. Van Beneden invece fa notare che mentre sono notevolmente comuni i Cecrops che invadono addirittura le branchie di certi individui del pesce sopra detto, soltanto raramente

(i) Il caligide che è stato citato sotto questo nome da me per la Liguria (1), ( $1898, \mathrm{p} . \tau_{3}$ ), devesi riferire alla Dinematura serrata di Kröyer. 
si osserva la presenza dei Laemargus. Non comune sarebbe questa forma, anche a detta di Van der Hœeven, nell'Atíantico.

Poche $\left({ }^{\mathrm{I}}\right.$ ) ha rilevato ultimamente che il nome di Laemargus (derivante dal

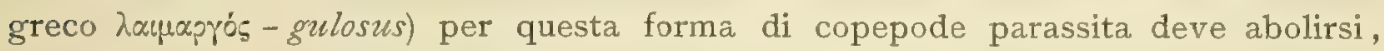
poichè esso è stato adoperato anteriormente da Müller e da Henle per stabilire un genere di pesci. Kröyer ha stabilito il suo genere nell' anno 1837 , e in verità nel quinto fascicolo dell' annata consistente in 6 fascicoli, per cui senza dubbio non prima di Settembre. Quasi nello stesso tempo invece da Müller e da Henle fu stabilito un genere di squalo, Laemargus, e precisamente nel « Ber. Verh. Ak. Wiss. Berlin», Juli 1837, che per conseguenza ha la priorità sul Laemargus Kröy. Per ciò il Poche credette necessario per il Laemargus di Kröyer di sostituire un nuovo nome, e scelse tale denominazione dall' unico ospite soltanto conosciuto di questo copepode parassita, ossia dall' Orthagoriscus mola fece il genere Orthagoriscicola nom. nov., per cui d'ora innanzi la specie viene distinta conseguentemente coì termini di O. muricatus (Kröy.).

Alcuni ittiologi, in questi ultimi anni, supponendo invece che il dritto di priorità dovesse spettare al genere di Kröyer, sostituirono al nome Laemargus di Müller e Henle quello di Somniosus (Lesueur) per designare il genere di squalo, di cui due specie sono note e designarono quindi le due forme conosciute di questo pesce in tal modo: Somniosus rostratus, ricordato da Risso e Canestrini pel Mediterraneo, colle località di Nizza e Genova e il Somniosus microcephalus (Schneider) Goode and Bean pel Nord Atlantico. Tale citato troviamo nel lavoro di Groode e Bean «Ittiologia Oceanica », Washington.1895, e anche nel recente manuale dell'Hœpli «Ittiologia Italiana * dove il Griffini ha adoperato pure il termine Somniosus (Lesueur) invece di Laemargus.

Per quanto è stato detto, pel diritto cioè di priorità rilevata dal Poche in favore del genere Laemargus di Müller et Henle, tale sostituzione compiuta dagli ittioligi per lo squalo non avrebbe più ragione di sussistere.

\section{Gen. Perisgopus Steenstrup et Lürken,}

\section{Perissopus dentatus SteEnstrup et LütKen, $q$.}

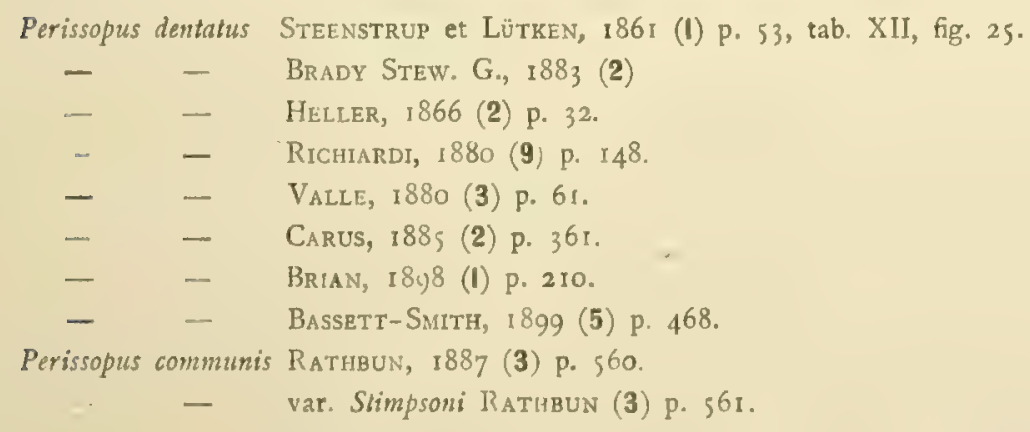

IIabitat. - Pel Mediterraneo questa specie non sembra essere rara e fu trovata sulla cute di squali diversi. Richiardi la rinvenne sulla pelle di Mustelus

(1) Poche Franz, - Bemerk, z. d. Arb. des H. Bassetl-Smith; ig02, p. i4. 
equestris Bp. A Genova furono raccolti esemplari sulla coda del Galeus canis Linn., il 7 Novembre 1892, sulla pelle di Mustelus laevis M. e H., il 2 Marzo 1893 e sul corpo di una Carcharias lamia Risso, il 20 Settembre 1898 . Heller e Valle ricordano questa stessa forma per l'Adriatico ove la riscontrarono sul Mustelus plebejus Bp. e sullo Squalus Milberti Bp.

La forma tipica studiata da Steenstrup et Lütken fu rinvenuta nell'Atlantico sopra di una sp. di Carcharias. Rathbun cita il Perissopus communis sinonimo del nostro, come proprio di parecchie località dell'America settentrionale e parassita di quattro forme diverse di pesci: Carcharinus obscurus; C. Milberti; Reniceps tiburo; Scoliodon Terrae-novae. La varietà Perissopus Stimbsoni del nostro copepode fu pure raccolta nell'America del Nord e l'ospite suo, secondo Rathbun, è ignoto.

La specie $P$. dentatus è dunque 1'unica del genere che pure mostrando un'area di distribuzione assai ampia vive in Europa, giacchè le altre sono esotiche. Il Perissopus (Lepidopus) armatus di Dana, come è noto, fu raccolto su di una specie di Mustelus proveniente da Rio Janeiro e il P. incisus di Van Beneden, fu tolto da non si sa quale pesce del Senegal.

\section{Fam. III. - PHILICHTHYTDAE.}

\section{Gen. Philichthys Steenstrup,}

Philichthys Xiphiae Steenstrup, $q$ e $\sigma^{7}$

Philichlbys xiphiae Steenstrup, r86r (1) p. 295, pl. II; 1862, p. 227.

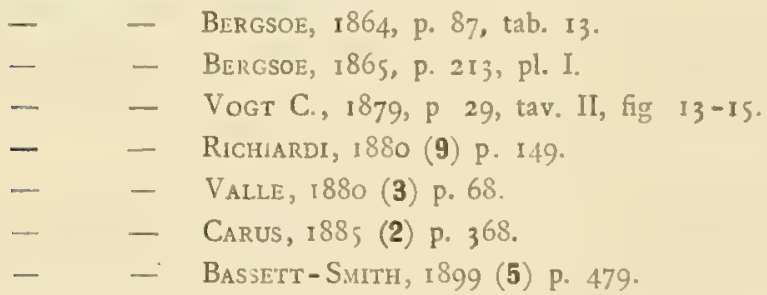

Distributio et habitat. - Questa specie vive nei seni e canali delle ossa frontali di Xiphias gladius Linn. ed è citata pel Mediterraneo da Richiardi e per l'Adriaticn dal Valle.

Steenstrup aveva pel primo scoperto questo copepode, di cui Bergsoe ha fatto una descrizione dettagliata. Quest' ultimo in un suo viaggio in Italia si è occupato dello studio di tale parassita che trovò comunissimo; sopra quindici teste di Xiphias gladius ha raccolto quarantanove femmine e ventun maschi, e ne ha riscontrati persino sette individui sopra una sola testa; è merito di Bergsoe collo studio accurato che ne potè fare d'aver assodato per primo che i Philichthys sono veramente crostacei appartenenti alla sezione dei copepodi parassiti.

Gen. Polyrrhynchus RichIARd,

Polyrihynchus Sciaenae Richiard, $q$ e $0^{7}$.

Plitichtbys Sciaenae RICHIARDI, I876 (2) p. $19 \mathrm{I}$.

$$
\begin{array}{lll}
- & - & \text { RiChIARDI, I880 (9) p. I49. } \\
- & - & \text { VOGT, I877 (2) p. } 412 . \\
- & - & \text { CARUS, I885 (2) p. } 368 .
\end{array}
$$

Richiardia Scialnai BASSETT-SMITH, 1899 (5) p. 479. 
Habitat. - Richiardi rinvenne questa specie nei canali delle squame della regione caudale della Sciaena aquila Lac. Mediterraneo.

\section{Poljrrhynchus Lichiae Richiardi, $\subsetneq$.}

Philichthys Licisiae Richiard, 1877 (5) p. 167, tav. VI, fig. ז.

- - Richiard, 1880 (9) p. 149.

- $\quad$ - VALLE, I880 (3) P. 4.

- $\quad$ - Carus, I885 (2) p. 369.

Richiardia Lichiae BASSETT-SMiTH, I899 (5) p. 478 .

Habitat. - Richiardi trovò questa specie nei seni e canali delle ossa frontali e della mascella inferiore della Lichia amia Linn. Mediterraneo.

Valle ne rinvenne due soli esemplari su lo stesso pesce nell'Adriatico, viventi nei seni e canali delle ossa frontali.

Polyrrhynchus Denticis Rtchiardi,.

Philichthys Denticis Richiard, I 877 (5) tav. VI, fig. 2.

- - Richiard, I880 (9) p. I49.

- - Carus, 1885 (2) P. 369 .

Richiardia Denticis B.sSETT-Smith, 1899 (5) p. 479, Pl. XXVI, fig. 3.

Habitat. - Questa specie è citata e descritta dal Richiardi. Vive nel Mediterraneo nei seni e canali delle ossa frontali e della mascella inferiore del Dentex vulgaris Cuv. e Val.

\section{Polyrrhynchus Pagri Richiardi, $९$.}

Philichthys Pagri Richiardi, I877 (5) tav. VI, fig. 3.

- - Richiardi, i880 (9) p. I49.

- - Carls, 1885 (2) p. 370.

Richiardia Pagri BASSETT-SмITH, I899 (5) p. 478.

Habitat. - Richiardi trovò questa specie nei seni e canali delle ossa frontali del Pagrus vulgaris Cuv. e Val. Mediterraneo.

\section{Polyrrhynchus Pagelli Richiardi, $q$.}

Philichthys Pagelli Rtchiardi, I877 (5) tav. VI, fig. 4.

- - Richiardi, i88o (9) p. 149.

- $\quad$ - Carus, i88j (2) p. 370.

Richiardir Pagelli BasseTt-SMith, I8yg (5) p. $74^{8}$.

Habitat. - Richiardi raccolse questo copepode nei seni e canali delle ossa frontali del Pagellus mormyrus Cuv. e del P. erythrinus Cuv. Mediterraneo.

Polyrrhynchus Baraldii RrchIardr,

Philicilthys Baraldii Richiardi, I877 (5) tav. VI, fig. 5.

- - Richiardi, i88o (9) p. I49.

- - Carus, i885 (2) p. 370.

'Richiardia Baraldii Bassetr-SMIth, 1899 (5) p. 479.

Habitat. - Richiardi osservò questa forma di Philichthys nei seni e canali delle ossa frontali e della mascella inferiore della Chrysophrys aurata Linn. Mediterraneo. 
Polyrrhynchus Sieboldii RICHIARDr, ९.

Philichthys Sieboldii Richiardi, 1877 (6) tav. X, fig. I.

$$
\begin{aligned}
& \text { - } \quad \text { - Richiardi, I880 (9) P. I49. } \\
& \text { - } \quad \text { - Carus, I885 (2) p. } 368 .
\end{aligned}
$$

Habitat. - Questa specie è citata pel Mediterraneo dal Richiardi come parassita del Box boops Linn. dove alberga nei seni e canali delle sue ossa frontali. È piuttosto raro.

Polyrrhynchus minimus RichIARDI, 9.

Philichthys minimus RICHIARDI, 1877 (6) p. 882 , tav. X, fig. 2.

$$
\text { - - Richiardi, } 1880 \text { (9) P. I49. }
$$$$
\text { - - Carus, } 1885 \text { (2) p. } 368 .
$$

Habitat. - Richiardi raccolse detta forma nei seni e canali delle ossa frontali del Serranus hepatus Linn. Mediterraneo.

Polyrrhynchus Grubei Richiard, $q$ e $\sigma^{7}$.

Philichthys Grubei RICHIArdI, I877 (6) p. I83, tav. X, fig. 3-4.

- - Richiardi, i880 (9) p. I49.

- Carus, 1885 (2) p. 368.

Habitat. - Richiardi trovò questa specie nei seni e canali delle ossa frontali del Sargus annularis Linn. Mediterraneo.

Polyruhyehus Agassizii Richiardr, $\supsetneq$ e $\sigma^{7}$

Philichthys Agassizii Ruchiard, 1877 (6) p. 185 , tav. X, fig. 5.

$$
\begin{array}{lll}
- & - & \text { Richiardi, I880 (9) p. I49. } \\
- & - & \text { VALLE, 1884 (5) p. } 3 . \\
- & - & \text { Carus, 1885 (2) p. } 369 .
\end{array}
$$

Habitat. - Questa specie fu trovata da Richiardi nei seni e canali delle ossa frontali del Charax puntazzo Linn. nel Mediterraneo.

Valle la dice comune nell'Adriatico dove pure alberga nei seni frontali del pesce ora nominato.

\section{Polyrihynchus Haeckelli Richiard, $\subsetneq$.}

Philichthys Haeckelii Richiardr, I 877 (6) p. 187 , tav. X, fig. 6.

$$
\begin{aligned}
& - \\
& -\quad \text { Richiardi, I880 (9) p. I49. } \\
& -\quad \text { Carus, is8s (2) p. } 369 .
\end{aligned}
$$

Habitat. - Richiardi scoperse questa nuova forma di Philichthys nei seni e canali delle ossa frontali e della mascella inferiore di Brana Rayi Bl. Schn. Mediterraneo.

Polyrrhynchus Muraenae RichIARDI, $q$.

Philichthys Muraena RichIard, 1877 (6) p. 189 , tav. X, fig. 7 .

$$
\begin{aligned}
& \text { - } \quad \text { - Richiardi, } 1880 \text { (9) p. } 4 \text { 49. } \\
& \text { - } \quad \text { - C CARUs, I885 (2) p. } 369 .
\end{aligned}
$$

Habitat. - Richiardi osservò questa specie nei seni e nei canali delle ossa frontali della Muraena helena Linn. Mediterraneo.

Polyrrhynchus Richiardii Valle, Philichthys Richiardii VALLE, 1880 (3) p. $8 \mathrm{I}$. 
Habitat. - Questa nuova specie, scrive Valle, è rarissima. Egli ne raccolse un unico esemplare, il ro Novembre $\mathrm{r} 880$, in un canale dell' osso preopercolare di una Box salpa Cuv. nell' Adriatico.

Polyrrhynchus Edwardsii RichIARd, $q$.

Pbilichthys Edwarlsi RIchiardi, 1876 (3) p. 197 Tav. VI fig. 4.

- $\quad$ - Richiardi, i880 (6) p. 149.

- $\quad$ - Carus, I885 (2) p. 368.

Richiardia Edwardsii BASSETT-SMIтT, I 899 (5) p. 478.

Habitat. -- Richiardi trovò questa forma nei seni e canali delle ossa frontali del Serranus cabrilla Linn. nel Mediterraneo. È specie comunissima.

Polyrrhynchus Steenstrupii RICHIARd, $q$.

Plitichthys Steenstrupii RıCHIs. RDI, I876 (3) p. I99, tav. VI, fig. 5.

-- $\quad$ - RICHIARDI, I880 (9) p. I49.

- $\quad$ - Valle, I880 (3) p. 68.

- $\quad$ - C CARUS, I885 (2) p. 368

Richiardia Steensirupii BASSETT-SMITH, I899 (5) p. 478 .

Habitat. - Questa forma fu trovata da Richiardi nei seni e nei canali delle ossa frontali del Mullus barbatus Linn. e M. surmuletus Linn., Mediterraneo.

Valle la rinvenne abbastanza comune nei seni e nei canali delle ossa frontali del Mullus barbatus Linn., nell' Adriatico.

Polyrrhynchus Doderleini RICHIARd, $९$.

Philichthys Dodetleini RICHIARDI, I883 (I3) p. 558-559

Habitat. - Vive sul Labrus turdus Bl. nei canali mucosi della base della testa, sopra l'ascella opercolare. Mediterraneo.

\section{Polyrrhynclus Stromatei RICHIARDI, $q$.}

Philichlhys fatolae RICHIARDI, I880 (8) p. 26.

- $\quad$ - C CARUS, 1880 , Zoologischer Anzeiger III, n. 48, p. 69.

Philichthys Stromatei Richiard, I880 (9) p. I 49.

- $\quad$ - Carus, 1885 (2) P. 368.

- - V VAlie, I884 (5) P. 2.

Habitat. - Richiardi scoperse questa specie nei seni e canali delle ossa frontali dello Stromateus fiatola Linn. nel Mediterraneo.

Sullo stesso pesce e fissata nella medesima parte fu rinvenuta dal Valle nell'Adriatico.

\section{Gen. Sphaerifer RICHIARDI,}

\section{Sphaerifer Corvinae Leydig, $\subsetneq$.}

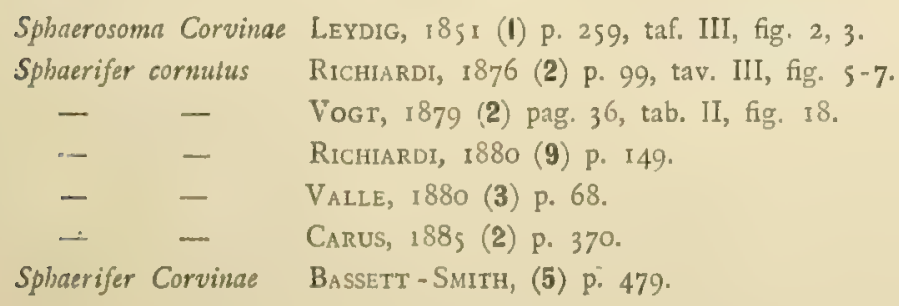


Habitat. - Richiardi raccolse questa specie sui seni della testa della Corvina nigra Cuv. e della Sciaena aquila Lac. nel Mediterraneo.

Valle la cita per l'Adriatico. È comune, e ne rinvenne, egli scrive, sino a sei esemplari in un testa di Corvina nigra di cui abita i canali e seni mucipari.

Fu notata per la prima volta a Cagliari da Leydig che la rinvenne nei canali mucosi di una Corvina sp.

\section{Sphaerifer Leydigii RICHIARd,}

Sphaerifer Leydigii RICHIARDI, I877 (5) p. I75, tav. VI, fig. 6-8.

- - Richiardi, I880 (9) p. 149.

- - VAlle, i880 (3) p. 69.

- - Carus, 1885 (2) p. 370.

- - BasseTt-SMith, I899 (5) p. 479, pl. XXVI, fig. 4.

Habrtat. - Questa specie si trova nei seni della testa dell' Umbrina cirrhosa Linn. e fu rinvenuta per la prima volta da Richiardi nel Mediterraneo e di poi dal Valle, che la dice comune nell'Adriatico e parassita di detto pesce.

\section{Fam. IV. - DICHELESTHIIDAE.}

\section{Gen. Anthosoma LEACH,}

Anthosoma crassum A BILDGAARD, $q$ e ơ. Tav. III, fig. I.

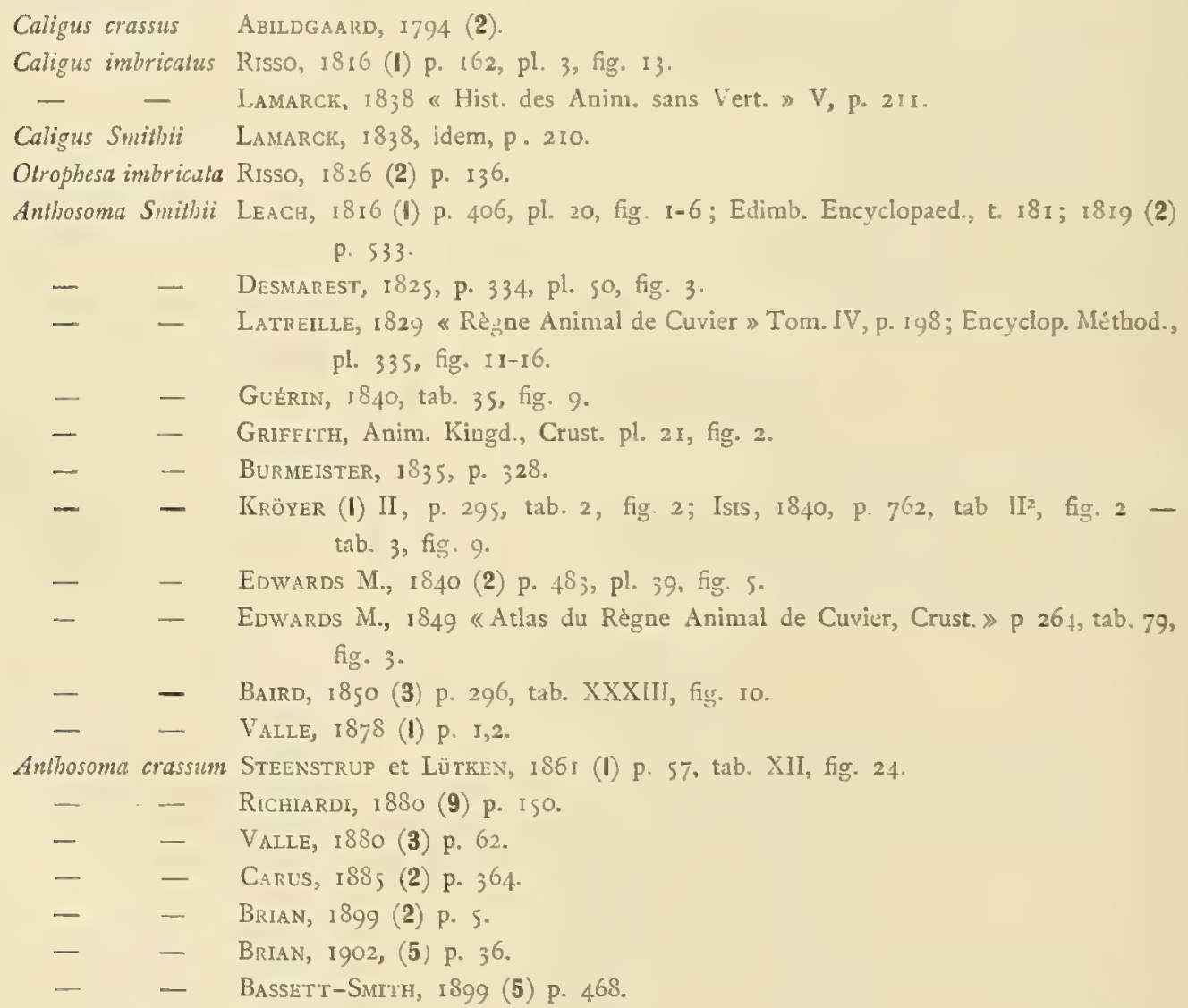


Distributio et habitat. - Questa specie di Anthosoma fu descritta da Leach da un esemplare raccolto sopra una Lamna cornubica Linn. (catturata in Exmouth, Devonshire) ch'era stato inviato da T. Smith al British Museum al D.' Leach. Detta specie fu rinvenuta pure nel Pacifico. Da Milne Edwards è citata sopra di uno squalo. Da Van Beneden è indicata altresì sulla pelle della Lamna cornubica Linn. per le coste del Belgio. Risso aveva riscontrato la sua presenza presso Nizza fin dal i8 16 . Nell'Adriatico, Valle la rinvenne abbastanza frequente situata fra i denti dell' Oxyrhina Spallanzanii Raf. Sopra dieci esemplari di questo pescecane, trovò otto affetti da questo parassita. Richiardi lo cita pel Mediterraneo affisso sulla mucosa boccale di siffatto ospite, sul quale sembra vivere preferibilmente nei nostri mari. Anch' io ne notai la presenza in Genova da un esemplare raccolto sulle branchie di simil pesce il 4 Luglio 1898.

Il D. Damiani me ne inviò un esemplare da Portoferraio da lui raccolto pure sulla mandibola di una Oxyrhina Spallanzanii Raf., il 20 Ottobre 1897.

\section{Gen. Lernanthropus NordmanN,}

Lernanthropus Trachuri BRIAN, ९. Tav. V, fig. 4, 5. Tav. XVII, fig. I.

Lernanthropus Trachuri BRIAN, IgO3 (9). p. 80.

Descrizione della femmina.

Questo parassita somiglia alquanto al L. Temminki di Nordmann, presenta però caratteri diversi e costituisce secondo me una specie nuova. Il mantello addominale è ampio, rigonfio nella sua parte posteriore bi -partito in due ampi lobi, in mezzo ai quali dal lato ventrale, al punto dove ha principio la divisione mediana in due parti, si distacca il post-addome lungo, gracile, con due strozzature, che danno allo stesso la sembianza di essere tri-articolato. Però il post-addome non sporge affatto al di fuori dei lobi del mantelio; mentre si prolungano invece ai due lati di quello e fuori di questo, quattro appendici strette, allungate, che, da sole, raggiungono la lunghezza di $\mathrm{I} \mathrm{mm}$. Il corpo $\mathrm{mi}$ sembra rivestito come da piccole granulazioni, che meriterebbero d'essere studiate, come già fece Heider per un altro Lernanthropus.

Degli esemplari da me esaminati uno arrivava, comprese le appendici addominali, a $3 \mathrm{~mm}$. di lunghezza, gli altri due a $3 \frac{3}{2}$ circa.

Distributio of habitat. - Varii individui furono raccolti dal D.r Damiani sulle branchie di Trachurus trachurus Casteln. a Portoferraio, il I8 Maggio Igor e il 22 Maggio 1903.

\section{Lernanthropus Mugilis BRian, $\subsetneq$.}

Lernanthropus Mugilis Brian, 1898 (1) p. 216 , tav. III, fig. 17.

$$
\text { - } \quad-\text { Brian; } 1903 \text { (9). p. } 80 .
$$

Descrizione della femmina.

La femmina finora osservata sembra presentarsi in uno stadio larvale non avendo potuto mai scorgere in questa forma i tubi oviferi. Il corpo è relativamente corto. Il cefalotorace esagonale, tanto largo quanto lungo, presenta ai lati 
due appendici coniche molto vistose. I suoi margini laterali sono ripiegati verso la parte ventrale. L'ađdome è assai largo e confusamente diviso in due parti. Il post-addome piccolo, bi-articolato è terminato da due foglioline caudali. Il terzo paio di piedi al disotto dell' addome è rappresentato da appendici brevi, fogliacee e ciascuna accartocciata in una sola piega longitudinale mediana. Queste due appendici sono avvicinate l'una coll'altra, e occupano non solo tutta la larghezza del torace, ma presentano una lieve sporgenza per ogni parte di esso.

Le quattro lamine caudali allargate (corrispondenti al quarto paio di piedi) nascono dal lato posteriore dell'addome e si prolungano di molto fuori del corpo e raggiungono quasi la lunghezza del cefalotorace. Esse ricoprono in parte il post-addome corto e rigonfio, che porta due appendici al termine, e che sta del tutto nascosto sotto la lamina a forma di scudo dell'addome.

Lunghezza del corpo $\mathrm{mm} .4 \%$.

Distributio et habitat. - Fu trovata la prima volta sulle branchie di Mugil auratus Risso, il 28 Ottobre 1889 a Genova: e sullo stesso ospite altri esemplari furono raccolti dal D. Damiani a Portoferraio nel rgoo; e altri ancora da me a Genova nell' Aprile 1898 sulle branchie di un Mugil indeterminato.

Lernanthropus vorax RICHIARDI, $q$ e $\delta$

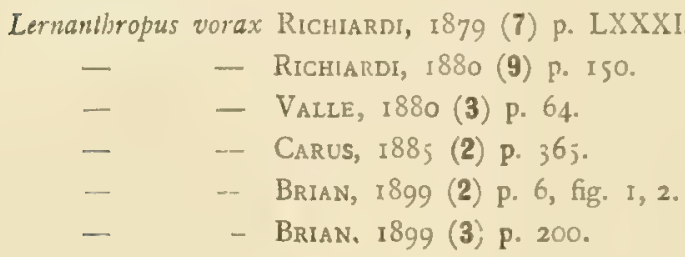

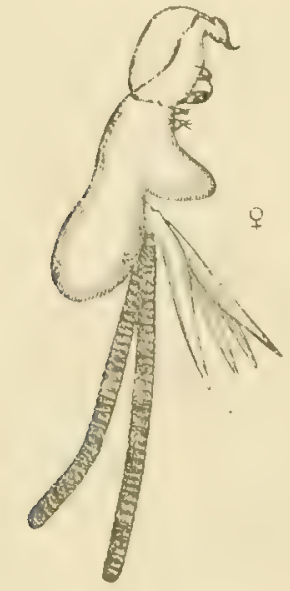

(ingr. $X$ II)

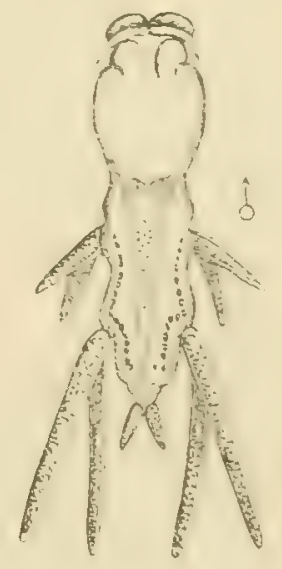

(ingr. $\times 2 \mathrm{I}$ )

Lernanthropus vorax RicE.

Questa specie fu descritta dal Richiardi brevemente nel 1879. Più tardi 1' ho fatta anch'io oggetto di esame e la illustrai in due mie note.

Distributio et habitat. - Più volte fu trovata aderente alle branchie di Charax puntazzo Linn. dal D.r Damiani a Portoferraio. Richiardi per il primo la trovò 
nel Mediterraneo su detto pesce, sul quale pure secondo Valle si trova di frequente fissata alle branchie, nell'Adriatico.

Lernanthropus brevis RICHIARDI, $q$ e ơ. Tav. XVII, fig. 3-5.

Lernantijropus brevis RICHIARDI, 1879 (7) p. LXXXI.

$$
\begin{aligned}
& \text { - - Richiardi i880 (9) p. I5O. } \\
& \text { - - VAlle, I880 (3) p. } 64 . \\
& \text { - - CARus, i885 (2) p. } 365 .
\end{aligned}
$$

Lernanlhropus Kröyeri var. HeIDER, I879 (I) p. 9 I wrong

Questa specie dall' Heider fu solo considerata come una varietà del Lernanthropus Kröyeri Van Ben. Si distingue dagli esemplari parassiti del Labrax lupus per il corpo più grosso e di struttura più robusta, per le antenne del primo paio, che tanto nel maschio come nella femmina sono indistintamente articolate (fig. 5); i rami dei piedi del terzo paio sono maggiori nel maschio.

Richiardi invece costituì di questa forma una specie distinta.

Una femmina da me esaminata a Napoli misurava la lunghezza di $2 \mathrm{~mm}$. comprese le appendici: il maschio arrivava appena a $\mathrm{mm}$. I ${ }^{\mathrm{x}} / \mathrm{z}^{-} \mathrm{I} 3 / 4^{\text {. }}$

Distributio et habitat. - Valle riscontrò questa forma comune sulle branchie di Sargus Salviani Cuv., di S. Rondeletii Cuv., dell'Oblata melanura Cuv., nel mare Adriatico. Heider in Trieste la trovò sulle branchie di Sargzs Salviani Cuv. e Val. Richiardi la notò pel Mediterraneo sulle branchie del Sargus Rondeletii Cuv. e Val. e $S$. annularis Cuv. e Val.

Il 6 Luglio I 903 ne rinvenni due individui (uno ơ e l'altro \&) sulle branchie di un Sargus vulgaris Groeff. a Napoli.

Lernanthropus scribae KRöYER, $q$ e $\sigma^{7}$.

Lernanthropus scribae KRÖYER, 1863 (2) p. 203, pl. IX, fig. 3.

- - HEIDER, 1879 (I) p. 86.

- - Richiardi, I880 (9) p. iso.

- - V VALLE, I880 (3) p. 63.

- $\quad$ - BasSetT-SMith, 1899 (5) P. 470.

Lernanthropus trigonocephalus HELLER, 1865 (1) P. 226, pl. XXII, fig. 3.

$$
\begin{array}{lll}
- & - & \text { Heller, I866 (2) p. 33. } \\
\text { - } & - & \text { Heider, } 1879 \text { (I) p. 85, fig. 67, } 68 .
\end{array}
$$

Distributio el habitat. - Questa forma, secondo Valle e Heider, è verosimilmente identica col L. trigonocephalus descritto dall' Heller. Le due provengono dal Serranus scriba Cuv. Valle dice rarissimo questo parassita che rinvenne sulle branchie di detto pesce nell' Adriatico. Heller lo cita sulle branchie dell'ospite ora nominato pell'Adriatico e per il Mediterraneo. Pochi esemplari mi furono inviati dal D. Damiani che li raccolse sulle branchie di Serranus scriba Cuv. rispettivamente il 9 Gennaio e il 23 Maggio I903.

Lernanthropus Gisleri BENEDEN v。, $q$ e $\sigma^{7}$ Tav. XVII, fig. 2.

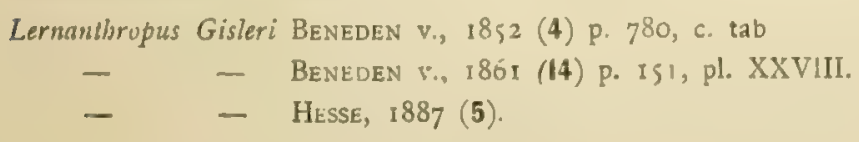


Lernanthropus Gisleri HeIder, 1879 (1) p. 83, fig. 65-66.

$$
\begin{aligned}
& -\quad-\quad \text { Richiardi, I880 (9) p I50. } \\
& -\quad-\quad \text { Valle, I880 (3) p. } 63 . \\
& -\quad \text { - Carus, I885 (2) p. } 364 .
\end{aligned}
$$

Lernanthropus Thompsoni BRIAN, I8g8 (1) p. 2I5, tav. III.

Lernanthropus Gisleri BRIAN, 1899 (3) p. 200.

$$
\begin{aligned}
& \text { - } \quad \text { - Brian, } 1902 \text { (5) p. } 41 . \\
& \text { - - Bassett-Smith, (5) p. } 472 \text {. }
\end{aligned}
$$

Distributio et habitat. - Van Beneden scoperse questa specie sulle branchie di Sciaena aquila Cuv. nel porto d'Ostenda. Hesse la rinvenne del pari su questo pesce nel mare al nord della Francia. Heider riscontrò la sua presenza in abbondanza a Trieste sulle branchie di Umbrina cirrhosa Linn. e di Corvina nigra Cuv. Richiardi la cita pel Mediterraneo con detti ospiti, e Valle la dice frequentissima nell'Adriatico pure su tali pesci. E stata da me notata anche pel mar ligure (Genova) sulle branchie della Lichia amia Linn., dell' Umbrina cirrhosa Linn. e della Sciaena aquila Lac, e per l'Isola d'Elba sul secondo di detti ospiti.

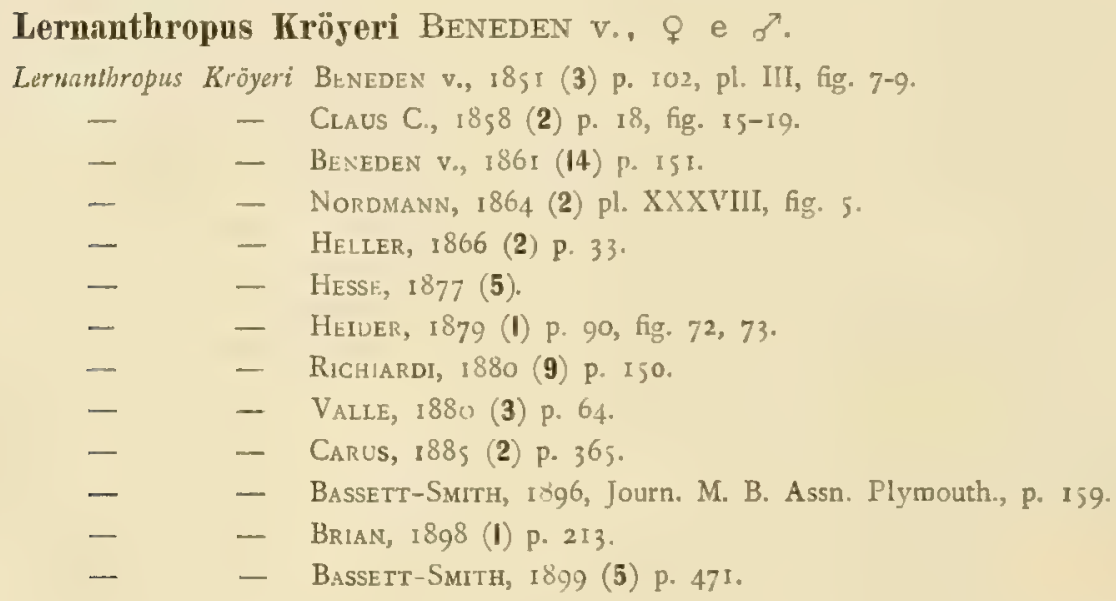

Distributio et habitat. - Vive sulle branchie del Labrax lupus Cuv. ed è citato per le coste del Belgio e per il mare al Nord della Francia, da Van Beneden, da Nordmann e da Hesse. Claus, che ne ha fatto oggetto di uno studio interessante a riguardo della sua morfologia e anatomia, lo ricorda per la località di Nizza. Per l'Adriatico è registrato da Heller, da Heider (per la località di Trieste) e da Valle; per il Mediterraneo da Richiardi e da me (per Genova). Bassett-Smith riscontrò la sua presenza in Inghilterra a Plymouth. È una specie di copepode che si trova quindi nei mari europei tanto nel Nord-Ovest, quanto nel Sud.

Lernanthropus Micropterygis RICHIARDI,

Lernanthropus Micropterygis RICHIARDI, I88; (14) p. 82-84.

Habitat. - Micropteryx Dumerili Risso. Palermo (?).

\section{Lernanthropus foliaceus RICHIARDI.}

Lernanthropus foliaceus RICHIARcI, I880 (9) p. I50.

$$
\text { - - Carus, i88s (2) p. } 365 .
$$


Specie non descritta. Richiardi la raccolse sulle branchie di Thyrsites pretiosus Cocco. Mediterraneo.

\section{Lernanthropus Tylosuri RICHIARDI,}

Lernantbropus Tylosuri RichiaRDI, 1880 (9) p. I5O.

$$
\text { - - Carus, } 1885 \text { (2) p. } 365 .
$$

Specie non descritta. Richiardi la trovò sulle branchie di Tylosurus imperialis Raf. Mediterraneo.

\section{Gen. Dichelesthium Hermann,}

Dichelesthium sturionis HERMANN, $q$ e $\sigma$.

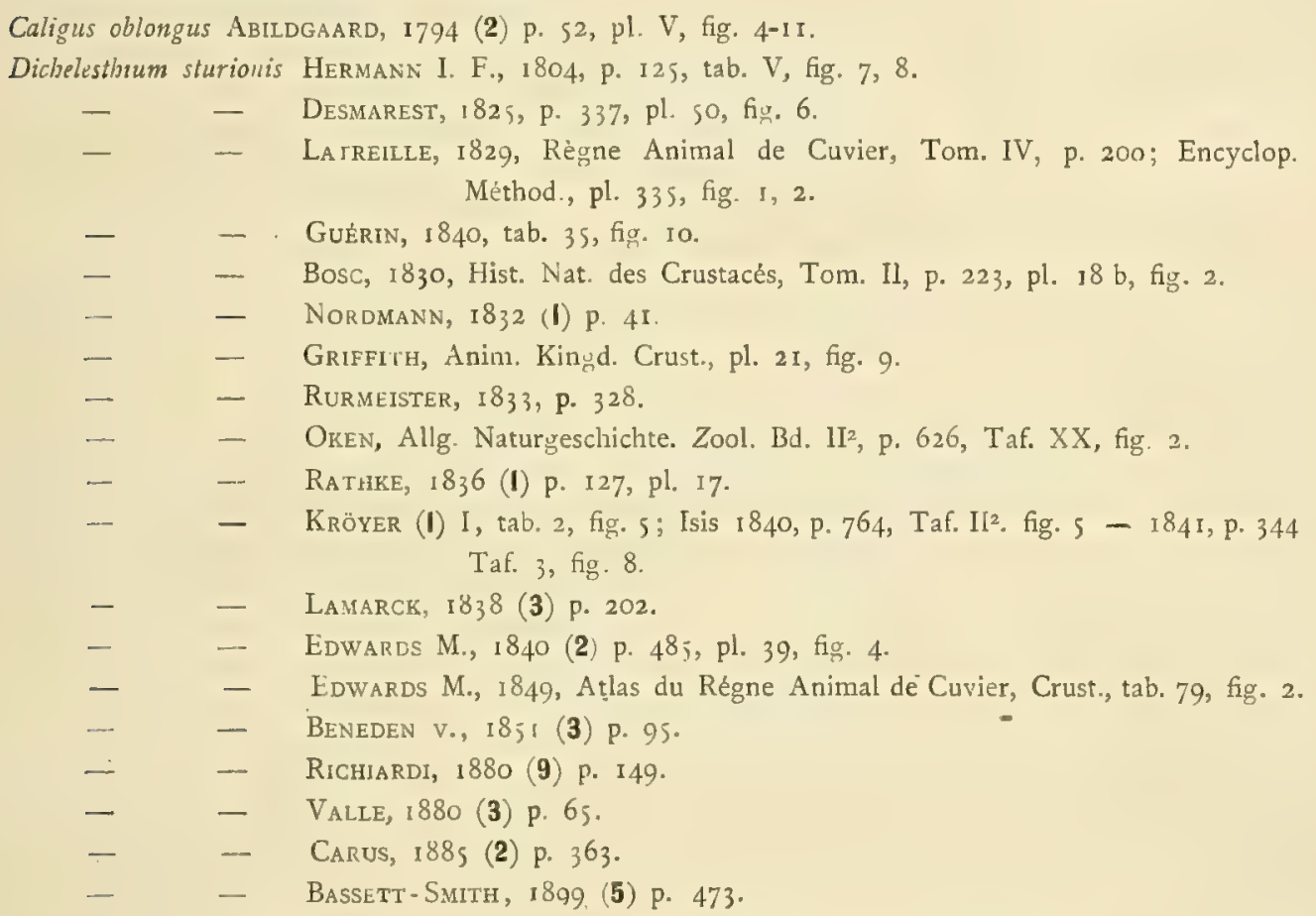

Distributio et habitat. - 亡 una forma assai nota e comune lungo i lidi al Nord dell' Europa. Vive parassita sull'Acipenser sturio Linn. E stata citata anche pel Mare Nero. Valle la ricorda per 1'Adriatico, alquanto rara, sopra le branchie del pesce ora riferito. Richiardi la cita parimente nel suo Catalogo come parassita delle branchie di Acipenser sturio Linn. e A. Nacarii Bp. Anch' io potei esaminare pochi esemplari raccolti dal $\mathrm{D}$. Giacomo Cecconi, nel 1903, sopra di un Acipenser sturio Linn. catturato a Fano (Adriatico), e inviatimi a Genova.

\section{Gen. Kröyeria BENEDEN v.}

Kröseria lineata BENEDEN v., q e o. Tav: V, fig. 3 .

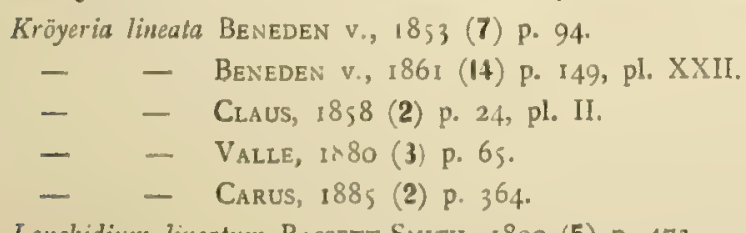

Lonchidium lineatum BASSETT-SMITH, 1899 (5) p. 473. 
Secondo Van Beneden la femmina è lunga circa $8 \mathrm{~mm}$. senza i fili oviferi, che da soli arrivano alla lunghezza di $7 \mathrm{~mm}$.; è larga un mezzo millimetro.

Gli esemplari femminili da me osservati a Napoli misuravano da 5,50 a $6 \mathrm{~mm}$. di lunghezza, e i fili oviferi erano relativamente più brevi, arrivando appena a $2 \mathrm{~mm}$.

Distributio et habitat. - $\dot{\mathrm{E}}$ citata da Van Beneden per il litorale del Belgio dove vive tra le lamelle branchiali di Galeus canis Linn. Claus pure la rinvenne a Nizza sulle branchie di questo pesce Valle la dice rara tra le lamelle branchiali di Mustelus equestris Bp. nell'Adriatico. A Napoli la rinvenni una volta sulle branchie di Galeus canis Linn. il 7 Luglio 1903.

\section{Kröyeria aculenta GeRSTAECKER, $q$.}

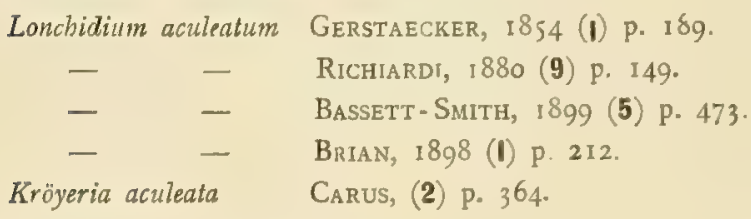

Questa specie è molto affine e forse identica alla precedente per quanto si può giudicare dalla descrizione che ci ha lasciato Gerstaecker.

Distributio et habitat. - Richiardi notò questa forma per il Merliterraneo, indicandola parassita del Prionodon glaucus Linn., e vivente sulla mucosa boccale e sulle arcate branchiali. Questa specie fu scoperta da Gerstaecker nell'Atlantico sopra di un pesce-cane. È stata da me citata per Grenova, osp. Galeus canis Linn.

\section{Gen. Hatschekia Poche ('). (Clavella OKEN).}

Hatschekia Sargi VALLE, ‡ Tav. III, fig. 2

$$
\begin{aligned}
& \text { Clavella ....... VAlle, I880 (3) p. } 85 . \\
& \text { Clavella Sargi VALIE, I882 (4). p. } 2 . \\
& \text { - - Carus, i885 (2) p. } 367 . \\
& \text { - - BrLan, } 1902 \text { (5) p. } 36 .
\end{aligned}
$$

Descrizione della femmina.

Il cefalotorace è breve assai mentre l'addome è allungatissimo. Quest' ultimo, osservato per trasparenza, lascia distinguere il tubo intestinale e gli ovari colle glandole cementarie il cui prodotto è espulso, come è noto, nello stesso tempo delle uova e forma l'involucro dei sacchi oviferi.

Si notano nel cefalotorace due paia di antenne, di cui uno è foggiato ad uncini; la bocca è a succhiatoio con piccole mascelle ai lati e inferiormente con un solo paio di piedi mascellari. Il torace presenta due paia di piedi natatori bifidi. Il maschio è ignoto. Gli esemplari misurano da I 1/2 a $2 \mathrm{~mm}$. di lunghezza senza contare i fili oviferi.

Distributio et habitat. - Valle trovò per la prima volta questa Clavella tra le branchie di Sargus Salviani C. V. nell'Adriatico a Trieste. Io ne rinvenni

(1) Il nome generico di Hatschekia è stato sostituito a quello di Clavella per soddisfare alle leggi di nomenclatura (vedi Poche Franz, r902) 
varii esemplari sulle branchie di Sargus sp., S. Salviani C. V. e S. Rondeletii Cuv. e Val. a Genova, nonchè di $S$. annularis Linn. a Napoli. Essa si trova abbastanza comune.

Hatschekia Richiardii GogGIO, $\subsetneq$.

Hatschekia Richiardii GoGGIO, IgO5 p. 222 fig. 4.

Habitat. - Sulle branchie di Labrus merula Linn. Mediterraneo.

Raccolsi alcuni esemplari di questa specie. a Napoli sul Labrus festivus Risso.

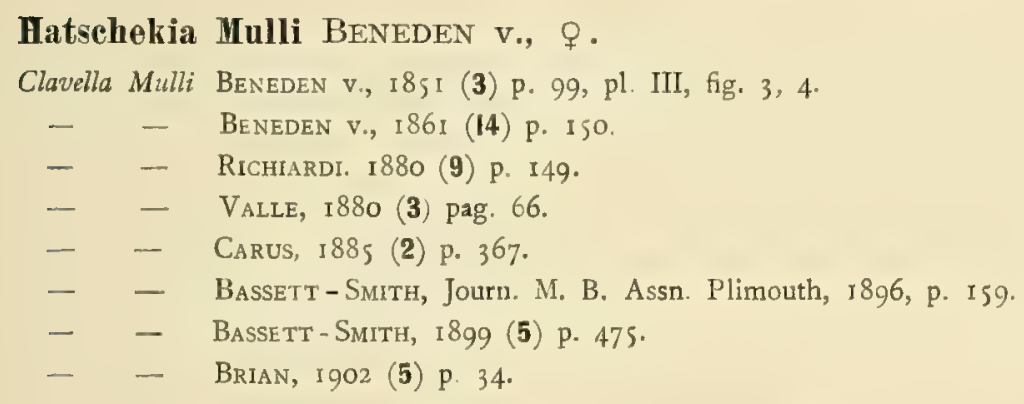

Distributio et habitat. - Da Van Beneden questa specie fu citata per le coste del Belgio sulle branchie di Mullus surmuletus Linn. Bassett - Smith la indica per le coste inglesi (Plymouth) come parassita di un Mullus sp.

Secondo Valle tale copepode per l'Adriatico è alquanto raro. Vive tra le lamelle branchiali del Mullus barbatus Linn. e Mullus surmuletus Linn.

Il Prof. Richiardi annunziava nei Processi Verbali della Società Toscana di Scienze Naturali in Pisa, I879, pag. LXXXII, di aver trovato fra le laminette branchiali degli ultimi due pesci ora detti, Mullus barbatus Linn. e Mullus surmuletus Linn., una Clavella che nominava Clavella crassa. Valle ritiene che non trattasi d'altro che della Clavella Mulli v. Ben., tanto più che quella specie non si trova registrata nel suo catalogo dei crost. par., 1880 , e difatti in questo lavoro è stata citata dal Richiardi solo la Clavella Mulli v. Ben. sulle branchie dei due Mullus surriferiti.

Dal D. Damiani me ne furono inviati alcuni esemplari raccolti sulle lamelle branchiali di Mullus barbatus Linn., il I Settembre I899, da Portoferraio.

\section{Hatschekia obesa RICHIARDI, $q$.}

Clavella obesa RichIARDI, I880 (9) p. I 49.

- - Carus, 1885 (2) p. 367.

- - Goggro, 1905, p. 217 , fig. I, Ia.

Habitat. - Tale copepode è stato raccolto da Richiardi sulle branchie della Muraena helena Linn. Mediterraneo.

Hatschekia (Cyenus Pagelli Bogupravei Hesse? \&.

Hatschekia Pagelli Bogueravei HESSE? 1879 (7) p. 14.

- - _ Goggro, 1905, p. 218, fig. 2, 2a, 2b, $2 c$. 
Habitat. - Sulle branchie del Pagellus centrodontus Cuv. e Val.; Rapallo I899 (molti esemplari).

Hatschekia Cernae GogGio, $\subsetneq$.

Hatschekia Cernae GoGGro, 1905, p. 219 , fig. 3.

Habitat. - Sulle branchie della Cerna gigas Cuv. e Val., Palermo I893.

Sulle branchie della Cerna aenea: Mediterraneo. (?).

Hatschekia Damianii n. sp.,

Clavella sp.? (Acantholabri exoleti HesSE)? BRIAN, 1902 (5) tav. I, fig. 16.

- $\quad-\quad$ - $\quad$ Brtan, igo3 (9) p. $8 \mathrm{r}$.

(Cycnus Acanblolabri exoleli Hesse)? I879 (7) p. IO, Pl. I9, fig. I7.

Hatschekia sp. ? GoGGro, 190j, P. 22 I.

Si veda la descrizione e la figura di questa specie nella mia nota citata IgO2 (J) p. 39 Tav. I., per quanto riguarda la femmina. Raccolsi i primi esemplari tipici che mi servirono per la descrizione, a Finalmarina il 27 Marzo 1897, sulle branchie di un pesce labrino indeterminato. Altri esemplari che mi sembrano simili mi giunsero da Portoferraio, raccolti sulle branchie di Crenilabrus paro C. V. nel Giugno rgor.

A questa specie è forse riferibile una forma di Hatschckia raccolta da Richiardi sul Labrus merula Linn. (Pisa) e che Goggio (1905, p. 22I) ritiene specie diversa dal Cycnus Acantholabri exoleti di Hesse, ma abbastanza somigliante coi miei esemplari, e alla quale non diede nome.

Hatschekia sp. Tav, III, fig. 3, 4. Tav. XX, fig. I-4.

Ultimamente ho trovato a Napoli sulle branchie di Crenilabrus pavo C. V. esemplari di Hatschekia di struttura non del tutto uguale a quella degli anzidetti individui di H. Damianii, per cui sono in dubbio sulla loro determinazione. Sono di dimensione notevolmente maggiore; mentre quelli misurano appena I millimetro, que sti arrivano a r, 6 e presentano uova discoidi. Riservandomi di studiare più tardi le particolarità e i caratteri di questa forma che non oso, per ora almeno, di dichiarare per nuova, ne descrivo intanto qui il maschio?? o almeno un individuo che ritengo per tale, assai diverso dalle femmine in mezzo alle quali l' ho trovato, e tanto di quello come di queste ne dò le figure disegnate sul materiale fresco.

Il solo individuo maschile rinvenuto (Tav. III, fig. 4) ha il corpo lungo circa $1 / 2$ millimetro: il cefalotorace più largo in proporzione dell'addome che non nelle femmine. L'addome per giunta mostra ai lati alcuni strozzamenti che ricordano la segmentazione primitiva.

Le lunghe antenne del primo paio sono costituite da una larga porzione basale munita di 5 o 6 spinule. Vistose pure assai più che nella femmina sono le antenne del secondo paio. (Tav. XX, fig. I, 2). Il primo e il secondo paio di piedi natatori presentano un articolo basale, come in quella, munito di due rami: l'interno è distintamente bi-articolato, l'esterno non sembrerebbe presentare con evidenza che un solo articolo (Tav. XX, fig. 4). 
L'addome finisce indietro restringendosi assai e termina con due piccolissime laminette caudali alquanto distanti l'una dall' altra.

Nelle femmine (Tav. III. fig. 3) il corpo è più grosso. In certi individui la larghezza dell'addome è assai rilevante e i lati vanno restringendosi molto poco, verso 1'indietro. Questi stessi lati in qualche esemplare mostrano un intaglio anteriormente e un' altro posteriormente. L'addome in avanti si restringe alquanto nel punto che sostiene le due paia di piedi biremi, in una specie di collo. Il cefalotorace è di poco più stretto ed è la sesta parte circa di tutta la lunghezza dell'animale.

Le setole all' estremità del ramo esterno del secondo paio di piedi, ováli-lanceolate, sono piuttosto larghe, specialmente quella mediana.

I tubi oviferi hanno uova grosse e discoidi.

Come dissi raccolsi un solo maschio (?) insieme a molte femmine, sulle branchie di Crenilabrus pavo C. V. a Napoli, 1'estate I903; e tanto esso come la femmina sono stati da me disegnati e dipinti allo stato fresco colle loro colorazioni naturali, prima di essere introdotti in formalina.

\section{Gen. Nemesis Risso,}

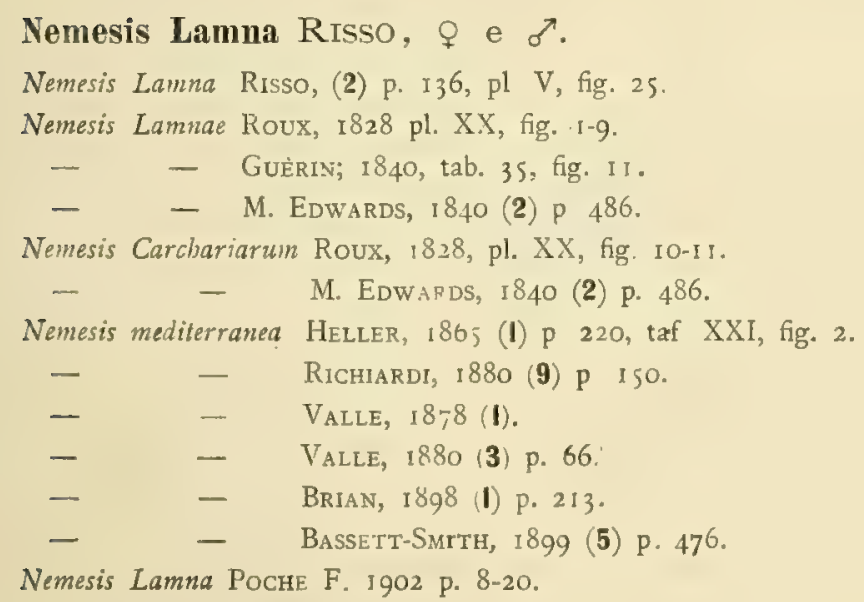

Distributio et habitat. - Valle cita 1a Nemesis mediterranea come propria alla fauna dell'Adriatico e la dice comune sulle branchie di Carcharodon Rondeletii M. e H. e di Oxyrhina Spallanzanii Raf. Richiardi per i nostri mari la ricorda nel suo catalogo come parassita delle branchie dell' Oxyrhina Spallanzanii Raf. e della Lichia amia Linn. Risso indicava questa specie sotto il nome di Nemesis lamna per Nizza, raccolta su di una Lamna cornubica Linn.; sopra di questo ospite e sopra di una Carcharias vulpes Cuv. è pure registrata per questa località dal Roux. È stata trovata in certo numero, in Genova, sull'Odontaspis ferox Agass.; sull' Oxyrhina Spallanzanii Raf. e sull'Alopias vulpes Linn. Heller notava la sua presenza pure nel Mediterraneo sopra di un pesce cane; sicchè vive esclusivamente, per quanto consta, nei nostri mari. 
A Portoferraio il D.r Damiani ne raccolse esemplari della varietà sinuata Valle, sulle lamelle branchiali d' Oxyrhina Spallanzanii Raf.

Nemesis lamna var. sinuata VALLE, . Tav. V, fig. 2.

Nemesis mediterranea var. sinuata VALLE, 1878 (I).

$$
\begin{aligned}
& -\quad-\quad-\quad-\text { Carus, i885 (2) p. } 365 . \\
& -\quad-\quad-\quad \text { Brian, i899 (3) p. 201. }
\end{aligned}
$$

Descrizione della femmina.

Questa varietà differisce, secondo Valle, dalla Nemesis mediterranea di Heller, per avere il cefalotorace non allungato e quasi quadrangolare come in questa ma bensì ovale, e per i segmenti addominali divisi tra loro da una profonda insenatura, che s'estende d'ambo i lati per un terzo della 1oro. larghezza. La larghezza di questi segmenti supera la lunghezza, per cui i tre primi assumono la forma d'un trapezio ad angoli bene arrotondati; mentre il quarto alla parte posteriore è quasi rotondo. Il colore del corpo è bruno verdastro.

La lunghezza totale del corpo è di $9 \mathrm{~mm}$, la larghezza 2, $5 \mathrm{~mm}$.

Distributio et habitat. - Valle la rinvenne sulle branchie di Oxyrhina Spallanzanii Raf., catturato lungo la costa istriana. Il D. ${ }^{\mathrm{r}}$ Damiani ne raccolse vari esemplari anche a Portoferraio, su di un tale ospite e sul Carcharodon Rondeletii M. e $\mathrm{H}$.

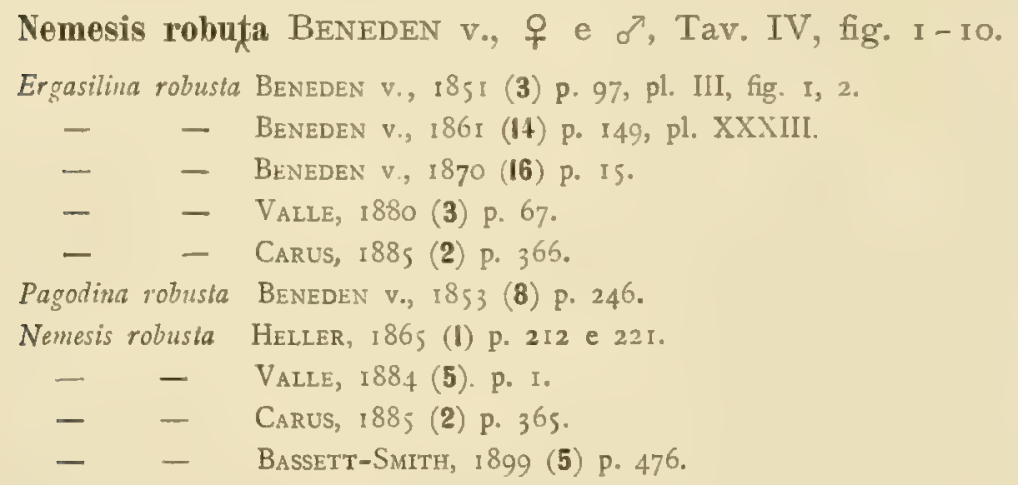

Van Beneden costituì di questa forma un genere nuovo che chiamò Pagodina perchè gli parve differisse nella sua forma, da tutti gli altri Dichelesthini e lo pose tra gli Ergasilus e i Dichelesthium. Heller per primo rilevò 1'affinità del genere Pagodina col gen. Nemesis, e notò che la specie di Van Beneden, ben a ragione era diversa dalla comune $N$. mediterranea. Secondo Bassett-Smith 1'Ergasilina robusta di V. Beneden è pure identica con questa forma.

La descrizione qui sotto data dei due sessi è stata fatta su esemplari da me raccolti nella Stazione Zoologica di Napoli.

Descrizione della femmina (fig. I-5).

Il corpo è molto largo verso mezzo. Il cefalotorace è perfettamente separato dall' addome, e risulta di un segmento di forma ovale e leggermente convesso al di sopra. L' addome è formato superiormente di tre segmenti, i quali ricoprono 
la parte superiore del corpo e sono assai larghi (fig. I, 2). L'ultimo segmento dell'addome che segue ai tre ora detti, e un pò meno largo. Quattro altri segmenti successivi costituiscono la regione post-addominale o caudale (fig. 3): i tre ultimi fra questi sono molto piccoli e assai avvicinati gli uni agli altri. L'ultimo porta due brevi appendici caudali.

Le antenne del primo paio sono molto sviluppate, inserite al di sotto del margine anteriore del cefalotorace e si mostrano distintamente pluri-articolate (I 2 articoli) e ogni articolo porta setoline. Inferiormente a queste si inseriscono le antenne del secondo paio, formate di quattro articoli. L'articolo basale è il più forte; il penultimo è alquanto più lungo; il terminale è leggermente uncinato e mostra due dentini sul margine concavo.

Il primo paio di piedi mascellari è relativamente forte; i suoi due articoli sono brevi e robusti; quello terminale porta all' estremità un uncino e alla base di quest' ultimo mostra ciuffi di peli.

Robustissimo è il secondo paio di piedi mascellari, (fig. 4) costituito da un breve articolo basale e da due altri più lunghi e robusti. L' ultimo è foggiato ad uncino, nella parte concava porta due dentini accostati verso la base e un altro più verso l' estremità libera.

Tutta la regione addominale presenta quattro paia di piedi natatori; il paio anteriore è piccolo, differisce completamente dalle paia seguenti; si compone d'una parte basale assai larga, con margine esterno tagliente e dentellato come una sega; di una lamina armata di tre artigli e di una spina alla base di essa. Le tre altre paia sono pressochè simili tra loro. Ogni piede è formato (almeno nel 2. paio, come ho osservato io stesso) di una grande lamina basale composta di due segmenti, quasi quadrata che porta due rami bi-articolati: il ramo interno è più forte dell'altro. L'ultimo articolo di ciascun ramo che è più piccolo, porta sei spine; mentre che il primo articolo del ramo interno porta una spina per ogni lato e quello del ramo esterno soltanto una. Queste appendici sono facili ad osser varsi al disotto del corpo e si muovono come natatorie, per un movimento lento dall' avanti all' indietro. Esse sono più svelte e più sviluppate nel maschio dove si notano setole piumose più lunghe.

Il quinto paio di piedi fissati ai lati del quarto anello addominale, è rudimentale, formato di un solo articolo breve con poche setoline all' estremità.

Le appendici caudali consistono di laminette ovali-lanceolate, terminate da tre brevi setole.

La bocca, a forma di succhiatoio, è ovale; vi si distinguono facilmente un paio di mandibole seghettate all'estremità e mascelle o palpi mascellari ai suoi lati, formati di due articoli e muniti di due o tre setole all'estremità.

I fili oviferi sono allungatissimi e sottili. Superano due o tre volte la lungezza di tutto il corpo e le uova sono disposte in una sola linea, avendo forma di dischetti, o di monete (fig. 5).

La lunghezza della femmina è di $5 \mathrm{~mm}$. senza i fili oviferi. Questi da soli arrivano a $13 \mathrm{~mm}$. di lunghezza. La misura di $5 \mathrm{~mm}$. è la stessa data da Van Beneden per la femmina della sua Ergasilina robusta.

Descrizione del maschio: (fig. 6-10). 
Il maschio è conformato presso a poco come la femmina; presenta una lunghezza quasi uguale, (variabile fra 4.50 e $5 \mathrm{~mm}$. ma è più stretto. Consta esso pure di non pochi segmenti (fig. 6-7).

Il cefalotorace in confronto cogli altri segmenti del corpo è relativamente grande; è leggermente convesso al di sopra e di forma ovale. Questo segmento come nella femmina è nettamente separato dagli anelli addominali.

L'addome è composto odi quattro anelli bene distinti, ma è da notarsi secondo Van Beneden che l' anteriore parimente come nella femmina è atrofizzato e nascosto al di sotto del segmento cefalico, ciò che risulta dalla inserzione delle quattro paia di zampe. I primi tre segmenti addominali sono più larghi che lunghi e ricoprono la parte superiore e laterale del corpo: essi sono ugualmente sviluppati. Il quarto è più lungo che gli altri e somiglia per la sua forma a quello del cefalatorace. Il corpo è in seguito formato di quattro altri segmenti più stretti che costituiscono il post-addome. Le appendici caudali inserite sull'ultimo, mostrano ciascuna, tre filamenti setiferi (fig. Io).

Le appendici del corpo somigliano presso a poco nella struttura a quelle già sopra descritte per la femmina, soltanto ho potuto constatare le differenze seguenti: che in quella le antenne del primo paio sono meno nettamente articolate, come pure le quattro paia di piedi natatori sono meno sviluppate e più rudimentali che nel maschio. Il quarto anello addominale nel maschio è piuttosto grosso. Vi si formano gli spermatofori voluminosi, ovali, coloriti in rosso bruno (fig. 9).

Distributio et habitat. - Van Beneden trovò questa specie sulle coste del Belgio dapprima sulle branchie di Trigon pastinaca Linn. e poi su quelle di Galeus canis Linn., di Carcharias glaucus Cuv. Valle la dice comunissima per l'Adriatico. Egli la rinvenne fra le laminette branchiali di Trygon thalassia Col., di Laeviraja oxyrhynchus Bp. e di Laeviraja macrorhynchus Bp., di Mustelus plebejus Bp. e di Mustelus equestris Bp. Ne raccolse pure grande quantità di esemplari tra le lamelle branchiali di un Notidanus griseus Cuv. Io, a Napoli, rinvenni due femmine e due maschi di questo copepode sulle branchie di Sphyrna zygaena Linn. il 24 Luglio 1903 e ne trovai un numero straordinario (da ricordare addirittura una polielmintiasi) sulle branchie di Alopias vulpes Linn., il 28 Luglio 1903.

Gen. Cycnus M. Edwards,

? Cyenus budegassae KRöYer, $q$.

Cycnus budegassae KRöYER, IS63 (2) p. 270 , pl. XII, fig. 3.

- - B BASETT-SMith (5) 1899. p 476

- - Carus, 1885 (2) p. 366.

Descrizione della femmina.

Per quanto $\mathrm{mi}$ è dato rilevare dalle figure date da Kröyer questa forma sembra assai stranamente e diversamente foggiata degli altri Cycnus, tantochè dovrebbe separarsi in un altro genere.

Presenta un cefalotorace rettangolare colla maggior lunghezza nel senso longitudinale. Segue una porzione di collo lunga presso a poco come il cefalotorace, 
più larga all'indietro, e di forma quasi triangolare, che porta per ogni lato quattro appendici somiglianti a spine poste ad uguale distanza l' una dall' altra. Segue all' indietro 1'anello genitale assai grosso, distintamente separato, più lungo da solo di tutta quanta la regione anteriore, ovale-allungato, e anteriormente assai più largo di quella, invece all' indietro va un poco restringendosi.

I1 margine anteriore del cefalotorace è intagliato nel mezzo. Sul lato ventrale di esso si vedono le appendici antennali e boccali. Le antenne del primo paio sono distintamente bi-articolate e a forma di uncino, fissate e sporgenti fuori del margine anteriore: ai due lati della fronte verso l'esterno sporgono fuori due piccole salienze a punta a guisa di brevi spine, produzioni dello stesso cefalotorace. Nel mezzo della parte anteriore si osserva la bocca, un piccolo succhiatoio rotondo e poco prominente, un pò in alto di esso e sotto le due antenne posteriori due piccolissime mascelle. Al di sotto di esse e ai lati del rostro due vistosi e uncinati piedi mascellari coll' uncino rivolto all' indietro.

Di piedi natatori si vede un paio piccolissimo verso la metà inferiore del cefalotorace: ciascun piede è bí-articolato. Dal confine posteriore dello stesso scudo cefalico spuntano fuori due altri tubercoli, evidentemente il secondo paio di piedi rudimentali.

Lunghezza della femmina $3 / 4$ di linea ossia mm. 1,62.

Habitat. - Kröyer notò la presenza di questa specie sulle branchie di Lophius budegassa Spin., nel Mediterraneo.

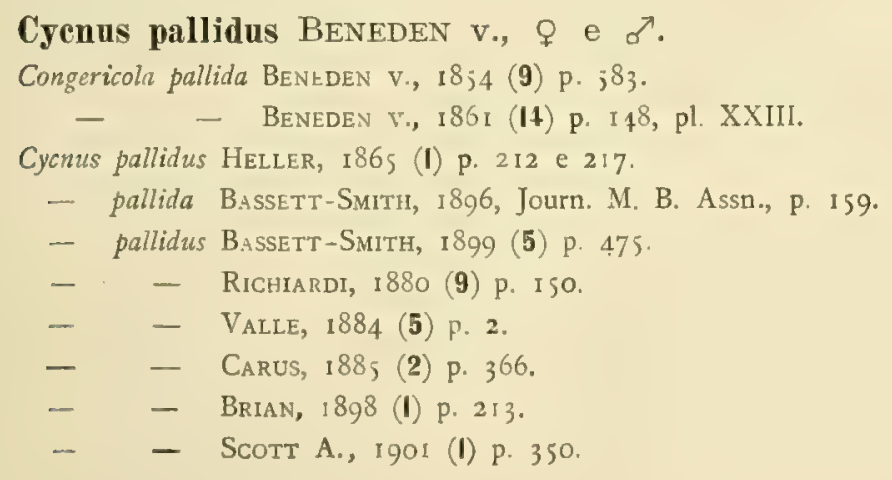

Łे una specie che presenta una certa quale somiglianza col Cycnus gracilis. Se ne distingue però a prima vista per avere il terzo anello addominale assai più largo che il primo e il secondo. Le appendici si presentano foggiate presso a poco ugualmente come nella specie or detta.

Van Beneden descrisse la femmina e il maschio. La lunghezza del corpo della prima è di $3 \mathrm{~mm}$. circa. I fili oviferi sottili e lunghi arrivano sino a mm. $5 \frac{1}{2}$. Nel seguente specchietto ho notate le misure rispettive delle varie parti della femmina, da un esemplare da me studiato nei dettagli e raccolto a Genova. 


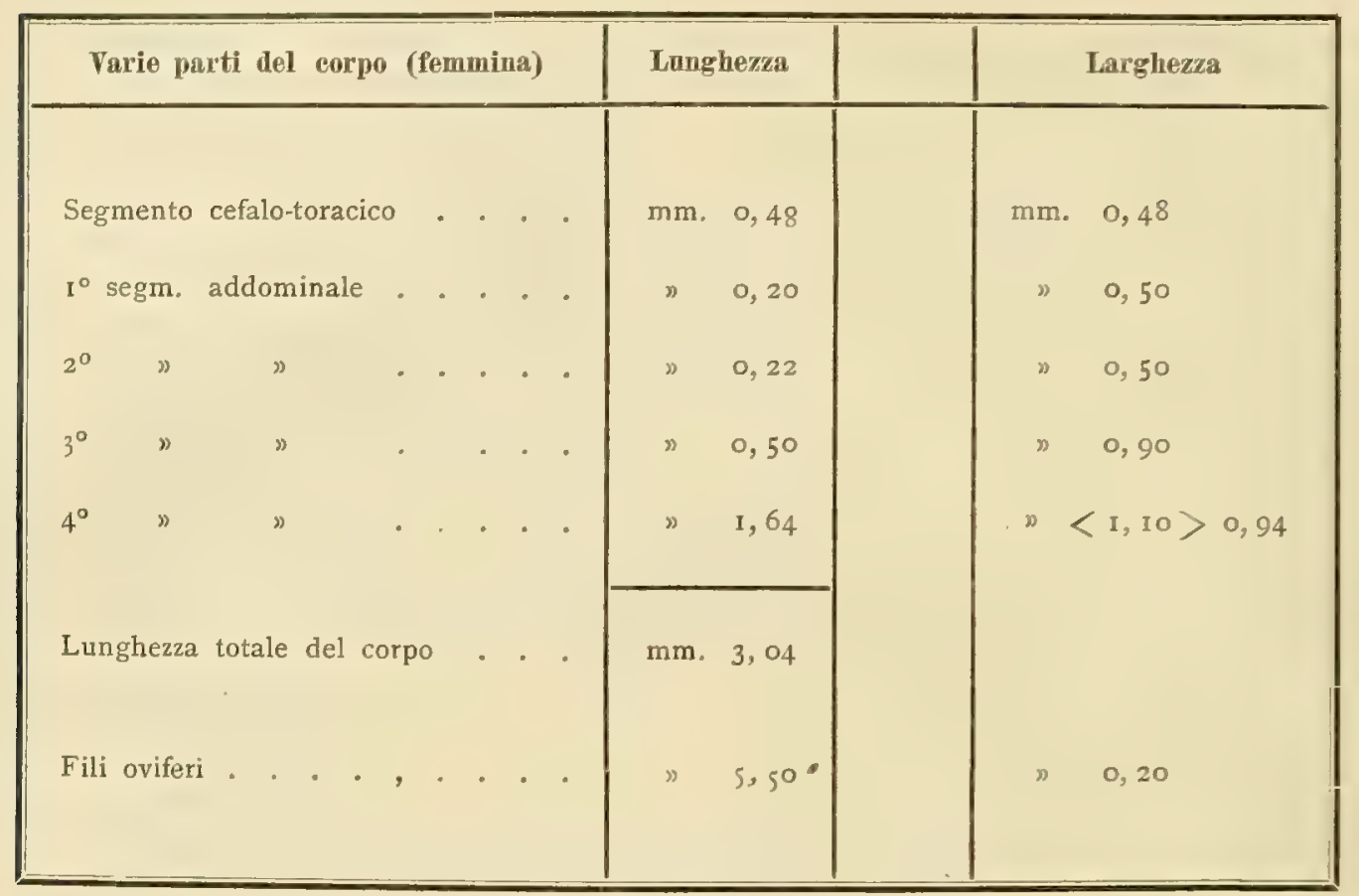

Distributio et habitat. - Van Beneden nota il Cycnus pallidus sulle branchie del Conger vulgaris Cuv. per le coste del Belgio e dice che non è abbondante: se ne trova di rado una mezza dozzina nello stesso pesce. Bassett-Smith lo indica pure sul Conger vulgaris Cuv. e come abitante i mari europei. Pel Mediterraneo è indicato dal Richiardi vivente tra le branchie dello stesso pesce; e per l'Adriatico da Valle, che lo ritiene alquanto raro.

Io pure ne constatai la presenza in Liguria sull' ospite sopra riferito.

Cyenus gracilis M. EDwARDS, $q$.

Cycnus gracilis M. EDWARDS, 1840 (2) p. 496, pl. 4I, fig. I.

$$
\begin{aligned}
& -\quad-\text { Heller, I86; (I) p. } 216 \text {, tav. XXII, fig. } 6 . \\
& -\quad-\quad \text { Heller, i866 (2) p. } 32 . \\
& -\quad-\quad \text { Valle, } 1880 \text { (3) p. } 67 . \\
& \text { - } \quad-\quad \text { Bassetr-Smith, I8g9 (5) p. } 475 .
\end{aligned}
$$

Distributio et habitat. - Milne Edwards ricorda questa forma come vivente sulle branchie di un merluzzo. Valle trovò questa specie tra le lamelle branchiali d'una Cerna gigas Bp. Heller l'aveva citata dapprima per l'Adriatico raccolta sopra un pesce non determinato.

\section{Gen. Pseudocycnus Heller,}

Pseudocyenus appendiculatus HeLler, $q$.

Pseudocycnus appendiculatus HelLER, I865 (I) p. 218 , pl. XXII, fig. 7 .

$$
\begin{aligned}
& \text {-- } \quad \text { - Richiardi, I880 (9) p. I50. } \\
& \text { - } \quad \text { - Carus, is8; (2) p. } 366 . \\
& \text { - - Bassett-SMith, (4) Ann. a Mag. N. H. ser. 7, vol. II. I8g8, p. } 368 . \\
& \text { - } \quad \text { - BASSETT-SMITH, } 1899 \text { (5) p. } 475 . \\
& \text { - } \quad \text { BRIAN, } 1902 \text { (5) P. } 45 .
\end{aligned}
$$


Distributio et habitat. - Gli esemplari originali descritti da Heller furono presi nell'oceano Atlantico su di una specie di Coryphaena. Altri esemplari di questo raro animale, scrive Bassett-Smith furono pure trovati attaccati alle branchie di Thynnus macropterus ad Aden; ed erano tutte femmine adulte.

Pel Mediterraneo è notata dal Richiardi sulla branchie di Thynnus pelamys Cuv. e Val. Io ne ho osservato esemplari raccolti dal Prof. Pavesi sulle branchie di Thynnus thynnus White, catturato all'isola Piana il I8 Giugno I884.

\section{Gen. Eudactylina BENEDEN v.,}

Eudactylina acuta BENEDEN v., $q$. Tav. V, fig. I.

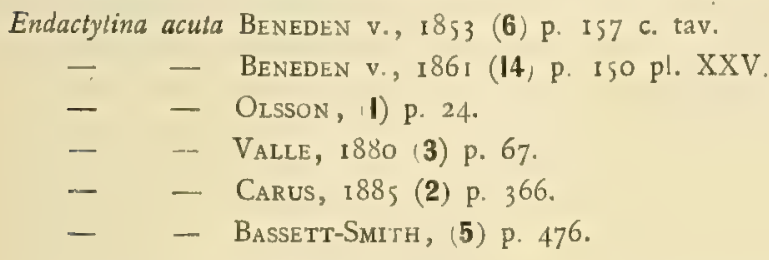

Descrizione della femmina.

Tutto il corpo è diviso regolarmente in segmenti nettamente separati gli uni dagli altri. Il segmento anteriore rappresenta il cefalatorace che è distintamente a forma di scudo, arrotondato all'indietro e che mostra sul davanti vistose antenne ricoperte di uncini; è un pò più largo indietro che in avanti, il mezzo si termina in un tubercolo. Dietro il cefalotorace vi sono quattro segmenti addominali di cui il quarto è più sviluppato; ciascuno di detti segmenti porta un paio di piedi bifidi. Il segmento genitale che segue è presso a poco simile al precedente: è un pò pì̀ allungato e porta indietro, a destra e a sinistra un'appendice munita di setole e di un articolo solo, il rudimento del quinto paio di piedi.

Il post-addome è formato di tre segmenti che diminuiscono di volume dall'avanti all'indietro. L'ultimo segmento porta un paio d'appendici caudali semplici.

I fili oviferi sono proporzionalmente corti, ma grossi e non contengono che un numero esiguo d' uova; Van Beneden ne conta da i3 a 15. Tali appendici sono inserite sul quarto anello addominale.

Di appendici sul corpo si contano dieci paia. Le due paia d'antenne sono molto forti e irte di spine; tri-articolate. Quelle del primo paio hanno un articolo basale assai grosso. La loro inserzione ha luogo al disotto e ai lati dello scudo cefalico presso il margine anteriore. L'articolo basale porta in avanti una forte spina ricurva, posta in mezzo di due altre dritte. L'articolo seguente terminale porta uncini invece di setole e l'ultimo soltanto setole.

Il secondo paio di antenne è foggiato in modo peculiare; è triarticolato, e il secondo articolo porta una forte spina inferiormente e piccole spine sull' estremità dell' articolo terminale.

Il primo paio di piedi mascellari è gracile e termina con un artiglio unico.

Alla base di questo paio e ai lati del rostro rotondeggiante e poco prominente, si notano le màscelle con due setole all' estremità una più lunga dell' altra. 
Il secondo paio di piedi mascellari è molto caratteristico: è robusto e termina con una punta trifida che va a poggiarsi nell' escavazione formata sul pezzo, opposto a guisa di pinze o chele.

I.e quattro paia di appendici addominali sono quasi simili e ugualmente sviluppate; ogni piede è composto di due rami tri-articolati e armati di setoline brevi. Il ramo interno termina a punta, l'esterno presenta diverse setole sull' estremità. Le appendici del quinto paio sono costituite di un solo ramo formato di un articolo unico munito di setoline.

Primo a descrivere brevemente questa specie è stato Van Beneden: di poi Olsson completò e corresse la descrizione che era stata data da quell' autore, con nuove osservazioni. A mo' d'esempio ha notato che le antenne anteriori in questa specie sono di sei o sette articoli, i mediani brevi e il primo e l'ultimo molto più lunghi. Nelle antenne posteriori il numero degli articoli gli sembrò essere di cinque(?). A me non è riuscito di scorgere un tale numero di articoli, e tanto per le prime come per le seconde antenne ho dovuto rilevare tre articoli soltanto.

Come pell' Eudactylina aspera di Heller ebbi a notare sulla superficie del corpo di questa specie piccole scabrosità a punta che Van Beneden non ha rilevato.

La lunghezza del corpo delle femmine è di $2 \mathrm{~mm}$. o poco inferiore; la larghezza di $1 / 2 \mathrm{~mm}$. Tale è la lunghezza assegnata da Olsson e così pure dagli esemplari da me raccolti a Napoli sulla Squatina angelus Dum. mentre Van Beneden attribuisce $6 \mathrm{~mm}$. alla femmina, compresi i fili oviferi. Ma questi ultimi negli esemplari da me esaminati appena sorpassano I mm., quindi si vede come la differenza sia grande.

Distributio et habitat. - Da Wan Beneden fu raccolta sulle coste del Belgio sulle branchie di due sp. di pesci Squatina angelus Dum. e Acanthias vulgaris Risso. Olsson trovò questa forma sulle branchie di detto Acanthias nel mare di Skagerrack il mese di Agosto. Egli prese 35 esemplari; circa 9 pesci su I4 ne erano infestati.

Valle dice questa specie comunissima per l'Adriatico e vivente tra le lamelle branchiali della Squatina angelus Dum. e dell'Acanthias vulgaris Risso. A Napoli la raccolsi pure su detti ospiti. (Estate Igo3).

A causa del colore e della piccolezza questo copepode sfugge facilmente alla vista dei raccoglitori; il colore negli animali viventi è bruno, l'intestino nero, mentre gli esemplari conservati in alcool hanno una tinta giallognola «flavescens».

\section{Gen. Lamproglena NORDMANN,}

Lamproglena pulchella NORDM.,.

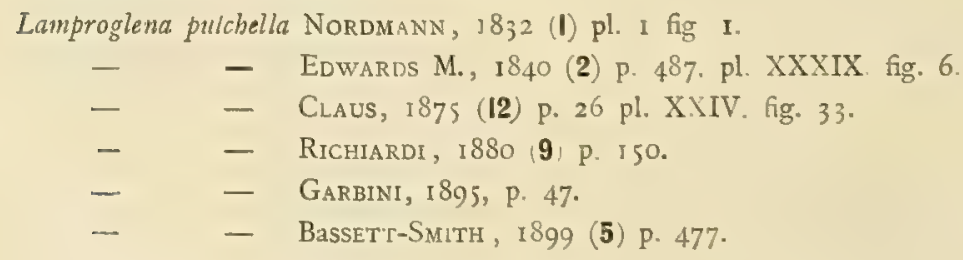

Questa forma fu descritta sufficientemente nel I 832 da Nordmann. 
Dopo più di quarant'anni da quell' epoca comparve il lavoro di Claus, che completò la conoscenza della femmina senza accennare al maschio, ancor oggi ignoto.

Distributio et habitat. - Vive nelle acque dolci d'Europa su diversi cyprinoidi. Da Nordmann è citata come albergante sulle branchie di Cyprinus jeses (Idus jeses Linn.) Richiardi la nota per 1' Italia sulle branchie dell'Alburnus alborella de Fil., Squalius cavedanus Bp., Telestes Savignyi Bp. e dello Scardinius erythrophthalmus Linn. Garbini riscontrò la sua presenza nelle acque del Veronese sulle branchie di Alburnus sp. e di Scardinius sp.

R. Issel, (Saggio sulla fauna termale ital.: Accad. reale delle scienze, Torino IgoI, p. 9.) né rinvenne esemplari presso Massa Marittima, nelle acque termali di Venelle, a $26^{\circ}$, parechi individui sulle branchie di un Telestes muticellus de Fil.

\section{Fam. V. - LERNAEIDAE.}

\section{Gen. Lernaeocera Blainville,}

Lernaeocera cyprinacea Linn. ૨.

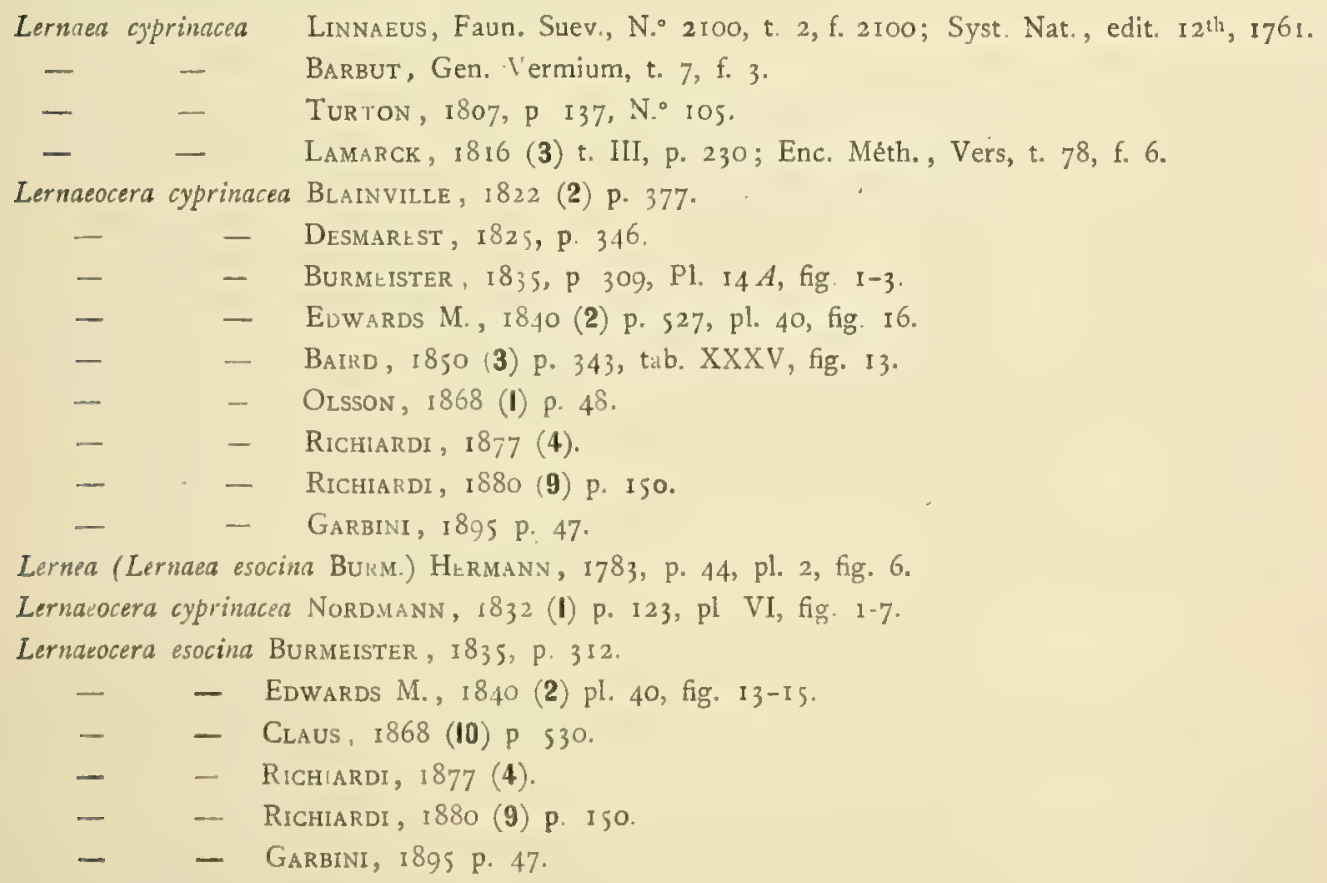

Non ho potuto avere nè esaminare esemplari di questa specie, sebbene si trovi anche in Italia vivente sui pesci d' acqua dolce. Appoggiandomi all' autorità di Bassett-Smith riunisco le due specie Lernaeocera cyprinacea e Lernaeocera esocina in una sola e mantengo per ragioni di priorità il nome assegnato a tale copepode da Linneo. Questo naturalista lo citava nel suo catalogo degli animali sotto il nome di Lernaea cyprinacea, trovato sul Cyprinus carassius Linn. (I76I). Nel I783 Hermann descrisse e disegnò col nome di Lernaea la stessa forma, che più tardí fu distinta da Burmeister col nome di Lernaeocera esocina. Il nuovo genere era 
stato fondato da Blainville nel I822. Dopo d'allora la Lernaeocera dei ciprinoidi da Burmeister e da Nordmann (1832) fu esaminata e fatta oggetto di accurati diseg'ni con migliori e più esatti mezzi d'osservazione, e a quelli noi oggi ancora dobbiamo ricorrere come a base fondamentale per la conoscenza di tale copepode.

Burmeister separò la Lernaeocera parassita del luccio dalla Lernaea cyprinacea di Linneo. credendo di vedere un carattere distintivo delle due forme nella diversità di grandezza; invece Nordmann le ritenne identiche ed oggi BassettSmith riconferma questa identità citandole come sinonime.

Distributio et habitat. - E specie comune e che si trova in tutte le acque dolci d'Europa, e citata per la Scandinavia, per 1'Inghilterra, per la Germania, per la Francia e 1'Italia ecc. come parassita di diverse specie di pesci: Cyprinus carassius Linn. (C. Gibelio Bloch); Cottus gobio Linn., Gasterosteus aculeatus Linn., Esox lucius Linn., Lota vulgaris Cuv., Abramis brama Flem. ecc

Vive fissata alla mandibola inferiore o alla pelle branchiale dell' Esox; abitualmente sugli altri pesci si rinviene profondamente fissata nei muscoli, in diversi posti, specialmente in vicinanza della natatoria dorsale, della linea laterale, dietro le natatorie ventrali e del petto, e la sua presenza dà luogo a tumefazioni o macchie di colore rosso vivo, da cui spunta fuori il parassita nella sua metà inferiore a mo' di appendice bruna, cilindrica, cogli ovisacchi; mentre la sua metà anteriore col capo, mediante le appendici cefaliche a mo' di croce, giace completamente nascosta nel tessuto. Per l'Italia questa specie è stata citata da Richiardi che, distinguendo le due forme di $L$. esocina e $L$. cyprinacea, nota la prima nelle masse muscolari dell' Esox lucius Linn., e la seconda nelle masse muscolari del Gobius fuviatilis Cuv.

Garbini, pel Veronese, cita parimente la prima sulle branchie di un Esox e l'altra sulle branchie e nei muscoli di un Cypirinus.

\section{Gen. Peniculus Nordmann,}

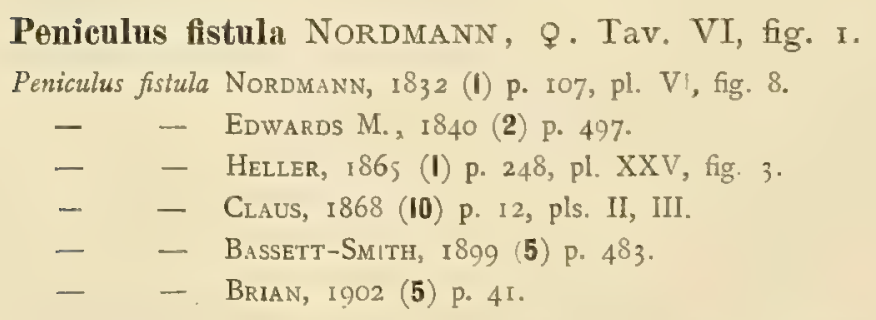

Dobbiamo la conoscenza di questo parassita scoperto dapprima da Rudolphi sulle lamine dorsali di Zeus faber, a Nordmann che lo descrisse col nome di Peniculus collocandolo, in aggiunta al genere Anchorella, tra i lerneopodidi. Più tardi Milne Edwards pose questo genere con altrettanto poco diritto accanto alla Clavella nei Chondracanthidae. Claus mostrò che è un lerneide e che deve essere riguardato come un tipo intermedio tra le lernee e i dichelestiidi.

La lunghezza dell' animale raggiunge, secondo Nordmann, linee $9^{1} / 2(\mathrm{~mm} .21,52)$ di cui $5 \% / 2$ linee ( $12,80 \mathrm{~mm}$.) comprendono i tubi oviferi e solo il rimanente riguarda il corpo; per contro la larghezza maggiore è appena di una mezza linea. Gli 
esemplari studiati da Claus provenienti dal Chilì, sebbene identici di forma presentavano una lunghezza inferiore di circa metà. Assai piccoli sono gli individui da me esaminati sebbene tutti adulti, come risulta dalle segnenti misure di tre esemplari raccolti a Napoli:

esempl. I. ${ }^{\circ}$ lunghezza corpo mm. $4 \frac{1}{2}$; tubi oviferi mm. $4 \frac{1}{2}$.

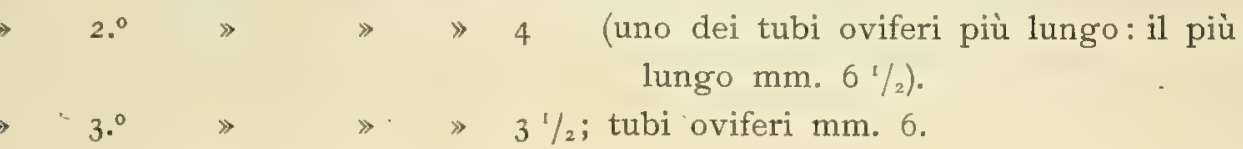

Il maschio è ignoto.

Distributio et habitat. - I primi ésemplari studiati da Nordmann vennero raccolti il 26 Luglio 18 I 7 a Napoli sulle pinne dorsali dello Zeus faber Linn. da Rudolphi che dette loro il nome di Dirhynchus fistula. Heller riscontrò questa specie nel Mediterraneo su di un pesce indeterminato. Claus fa osservare che il Peniculus clavatus somiglia assai alla nostra forma, ha per ospite il Sebastes norvegicus O. Fr. Müll. e fu descritto da Kröyer. Gli esemplari studiati da Claus vennero raccolti su di una Percis semifasciata del Chili. Come si vede dunque il Peniculus fistula ha un'area di distribuzione estesissima e per di più vive su ospiti molto diversi.

A Genova raccolsi due esemplari fissati sulla pinna caudale di Sargus Salviani C. V. (15 Febbraio Igor); a Napoli rinvenni 3 esemplari sulle pinne caudale e ventrale di Atherina Boyeri Risso (22 Luglio 1903).

\section{Gen. Pennella OKen,}

Pennella crassicornis Steenstrup et LütKen $q$. Tav. VI, fig. 2-7.

Pennella crassicomis Steenstrup et Lutken, I861 (1) p. 76, tab. XIV, fig. 34.

$$
\begin{aligned}
& \text { - - HelleR, 1865 (I) p. } 247 . \\
& \text { - } \quad \text { - Valle, i88o (3) p. } 69 . \\
& \text { - - Carus, i885, (2) p. } 373 . \\
& \text { - } \quad-\quad \text { BRIN, I9O3 (9) p. 8r. }
\end{aligned}
$$

Descrizione della femmina. (fig. 2-7).

Il corpo è allungato, cilindrico e il suo diametro trasversale è un po' maggiore nella metà posteriore che in quella anteriore. Il cefalotorace sull'avanti porta un grosso tubercolo verrucoso: alla base di esso per tre lati sporgono fuori altrettante appendici coniche assai lunghe arrotondate al loro termine. Le prime due sono in linea retta, la terza è ortogonale a queste, e spunta fuori sul lato dorsale; soltanto il lato ventrale resta privo di siffatto corno. Le appendici antennali e boccali riposano sulla prominenza anteriore del capo mentre le appendici rudimentali dei piedi natatori stanno nella parte posteriore del cefalotorace dietro la base delle appendici coniche, e sono fissate sul lato ventrale in numero di quattro paia.

Il colore del corpo nella parte anteriore è giallo sporco, nella metà posteriore, nerastro. Quell'ultima porzione assume un aspetto segmentato e porta una grossa 
appendice nera a guisa di penna, che è speciale alle Pennelle. I filamenti laterali all'asse di questa penna sono ramificati e il disegno è stato dato da Steenstrup e da Lütken nella figura 34 y tav. XIV. Da questi autori non è stata data la descrizione delle antenne nè quella delle appendici boccali, che restano quindi ancora da studiarsi per tale specie.

Le dimensioni variano a seconda degli individui. Un esemplare di grossezza media raccolto a Portoferraio e da me esaminato, misura 7 cent. circa di lunghezza e la maggiore larghezza del capo comprese le due corna laterali, e misurata fra i due apici delle stesse, arriva a r6 millimetri.

Nella seguente tabella ho dato le dimensioni delle varie parti del corpo degli esemplari raccolti dal. D." Damiani a Portoferraio. Ho distinto colla lettera $a$ la testa, con $b$ il collo, con $c$ la porzione rigonfia dell'addome e con $d$ la coda.

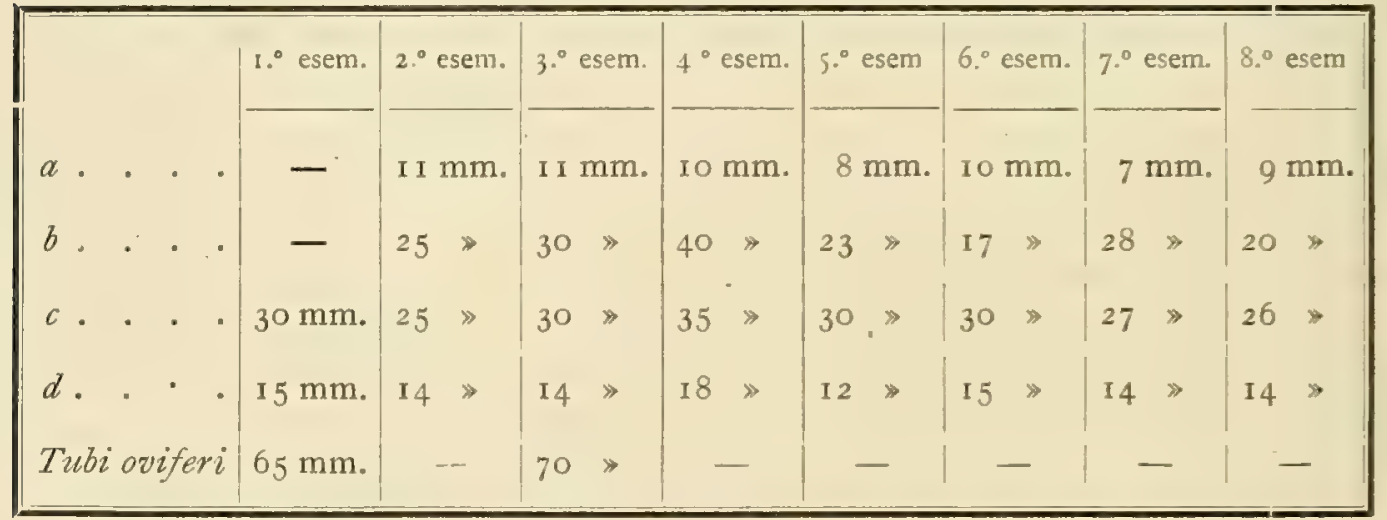

La lunghezza degli esemplari descritti e disegnati da Steenstrup e da Lütken, è di 80-Ioo $\mathrm{mm}$.

Distributio et habitat. - Heller cita semplicemente questa specie pel Mediterraneo senza indicare l'ospite. Valle per contro, citando tale copepode per l'Adriatico, aggiunge che raccolse due esemplari, uno fisso alla base della pinna anale d'un Naucrates ductor Raf,, e l'altro alla regione genitale di Xiphias gladius Linn. Il D. Damiani raccolse parecchi esemplari sulla cute del rarissimo Prodclphinus euphrosyne True, a Portoferraio, il 3I Luglio Igo2.

La Pennella crassicornis fu trovata per la prima volta da Steenstrup e da Lütken nell'Atlantico boreale, penetrante nella cute d'un Hyperoodon rostratus Müll.

\section{Pennella varians Steenstrup et LÜtKen,}

Pennella varians Steenstrup et Lüthen, I86I (I) p. 4r3, pl. XIV, fig. 32.

- pusiulosa? BaIrD, Ann. Nat. Hist. XIX, p. 280.

Pennella varians? WIERZEIJSKr, I 877 .

$$
\begin{aligned}
& -\quad-\quad \text { Carus, i88s (2) } 373: \\
& -\quad-\quad \text { Bassett-Smith, (5) p. } 483 .
\end{aligned}
$$

Distributio et habitat. - Si trova indicata per l'Atlantico, allo stato adulto, parassita sui delfini. Anche nell' Adriatico, a Trieste, ne fu notata la presenza da Wierzeijski, il quale avendo fatte ricerche sui giovani lerneini delle branchie dei cefalopodi (Sepia offic., Loligo vulg., Eledone mosch.) ha scoperto che la Pen- 
nella varians presenterebbe le stesse abitudini della Lernaea branchialis la quale a due epoche di sua esistenza separate dall'accoppiamento, predilige due modi di vivere differenti su due ospiti ugualmente differenti. La Pennella varians passerebbe la prima parte della sua esistenza parassitaria tra le branchie delle seppie e dei calamari, per trasportarsi in seguito sul delfino dove trascorre il periodo della produzione delle uova.

Pennella filosa Cuvier, $q$. Tav. XVIII, fig. I-7.

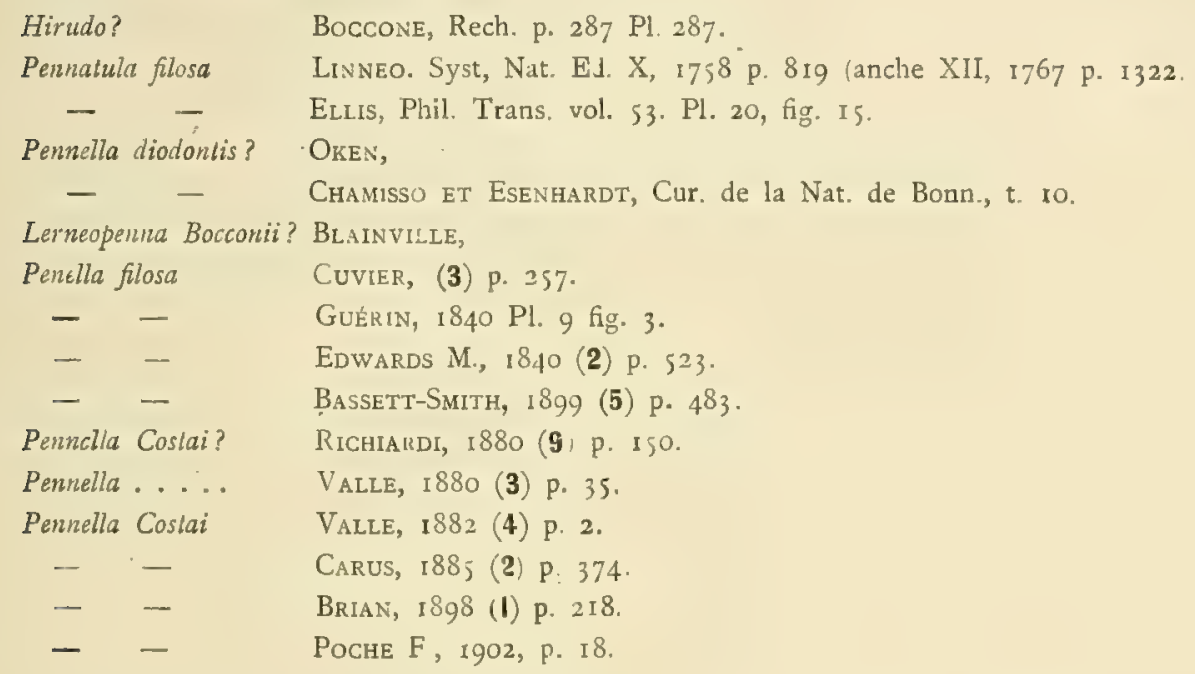

Descrizione della femmina adulta.

Richiardi citava nel suo catalogo di crostacei parassiti una Pennella che egli chiamò col nome nuovo di $P$. Costai, e che non ha descritto. La raccolse sullo Xiphias gladius Linn. nel Meditterraneo. Gli esemplari di Pennella che io qui sotto descrivo furono pure trovati sullo stesso ospite in Liguria: perciò ritengo che, data 1' identità d'ospite e di località, tanto gli esemplari di Richiardi come quelli da me esaminati possano riferirsi as una sola ed unica specie. D'altra parte due forme diverse sono citate dagli autori come viventi sullo Xiphias gladius. Una, la Pennella crassicornis (che però trovasi abitualmente come parassita anche di altri ospiti,) mi pare diversa, per alcuni caratteri, dai nostri esemplari; l'altra che ha nome Pennella filosa presenta invece con questi una certa somiglianza, ed anzi a giudicare dalla figura che ce ne dà Guérin, credo che quella specie e la nostra sieno uguali.

Gli individui da me esaminati e che si conservano nel Museo di Zoologia della $R$. Università di Genova non sono completi. A tutti è stata lacerata via la porzione cefalica, di certo, nell' operazione eseguita per toglierli dall' ospite. Ne risulta per conseguenza che ad essi manca. il capo colle antenne e colle parti boccali e la porzione di collo che dovrebbe conservare i resti delle quattro paia di piedi addominali: perciò la descrizione, qui sotto data, è incompleta.

Tutto il corpo ha una forma allungatissima e cilindrica. La porzione di collo è lunga in sommo grado, con tegumento liscio è di colore giallo sporco nella sua parte anteriore. Si allarga in modo graduale all' indietro e passa nell'addome rappresentato da un'altra porzione cilindrica, allungata, ma di diametro assai mag- 
giore, colorata in nero e divisa in inoltissimi anelli per effetto di una falsa segmentazione che non intacca il tessuto sottocutaneo.

La parte posteriore dell' addome prolungasi all' indietro in una porzione caudale più stretta, simulante essa pure una pseudo-articolazione per mezzo di strozzamenti laterali. È questa l'asse principale della penna o coda, dello stesso colore che l'addome, che porta ai due lati numerose appendici ramificate e formate a guisa di ciuff, ripiegate all' indietro. La ramificazione è complicata. Di solito su di uno stelo basale comune breve, è fissata in varie guise una serie di raggi biforcati e in modo che 1' uno dei rami è più lungo dell'altro.

Dalla base dell'appendice caudale si dipartono i fili oviferi sottili, lunghissimi, avvolti su sè stessi con grandi giri.

Taluno degli esemplari da me esaminati porta alla base della ora descritta appendice caudale, fissato fortemente sull'integumento, un grosso cirripede, che spetta al gen. Conchoderma. E questo un noto fatto di associazione o simbiosi constatato da Mayer e da Griard. (")

In questi stessi esemplari ho osservato per giunta altri simbionti nascosti tra i ciuffi di detta penna caudale, rappresentati da piccole colonie bellissime di Campanularia che insieme al detto cirripede sogliono convivere sulle Pennelle. Sembra che i casi di simbiosi di tale idroide sui crostacei parassiti dei pesci, non siano tanto rari, perchè il fatto fu già constatato da Agassiz per le Pennelle $\left({ }^{2}\right)$ e da Richiardi per un esemplare di Lernacenicus vorax e per cinque esemplari di Elytrophora brachyptera. (3)

Questa nostra specie di Pennella è di grandi dimensioni. La lunghezza media degli individui da me esaminati varia da I $_{3}$ a 15 centimetri ma taluni esemplari arrivano anche a 23 cent., sebbene non sia calcolata la regione anteriore del cefalotorace mancante in tutti questi esemplari e non sia tenuto conto dei fili oviferi.

Per rappresentare in modo giusto la rispettiva lunghezza delle singole parti costituenti il corpo, degli individui maggiori, riunisco nella seguente tabella le misure, intendendo, come sopra, di significare colle lettere $a, b, c, d$, le rispettive porzioni del corpo ossia la regione cefalica, il collo, l'addome e la regione caudale.

\begin{tabular}{|c|c|c|c|c|c|c|c|c|c|c|c|c|c|c|c|}
\hline \multirow[b]{2}{*}{$a$. } & & \multirow[b]{2}{*}{ - } & & \multicolumn{2}{|c|}{$1.0^{\circ}$ esempl. } & \multirow{2}{*}{\multicolumn{2}{|c|}{$\begin{array}{c}20^{\circ} \text { esempl. } \\
- \\
-\end{array}$}} & \multicolumn{2}{|c|}{$30^{\circ}$ esempl. } & \multicolumn{2}{|c|}{$4^{\circ}$ esempl. } & \multicolumn{2}{|c|}{$50^{\circ}$ esempl. } & \multicolumn{2}{|c|}{$60^{\circ}$ esempl. } \\
\hline & . & & . & & - & & & & - & & - & & - & & - \\
\hline 6. & . & • & • & 60 & $\mathrm{~mm}$. & & - & & - & I 20 & $\mathrm{~mm}$ & 77 & $\mathrm{~mm}$. & So & $\mathrm{mm}$. \\
\hline$c$. & . & - & - & 47 & $\gg$ & 50 & $\mathrm{~mm}$ & 38 & $\mathrm{~mm}$ & 75 & $\gg$ & 52 & $»$ & 53 & $\gg$ \\
\hline$d$. & . & . & - & 25 & $\gg$ & 30 & & 22 & » & 37 & $»$ & 30 & $»$ & 25 & $\gg$ \\
\hline
\end{tabular}

(1) Giard A. Sur l'Association de Pennella Orthagorisci Perceval et de Conchoderma virgatum Spengl. in: Le Naturaliste (2) I I Ann. No 50, p. 82 I $88 \mathrm{~g}$.

(2) Sulle Pennelle AgAssiz ha trovato la Campanularia (Eucope parasilica); v. Catalogo Illustrato del Museo di Zoologia Comparata. Cambridge, 1865, p. 87

(3) Richiardi rinvenne sui copepodi parassiti sopracitati la Campanularia Jolnstomi; vedi Richiardi: Descrizione di due sp. n. di Lernaeenicus, p. I2. 
I fili oviferi misurano la lunghezza da 50 a $60 \mathrm{~mm}$.

Distributio et habitat. - 'L' ospite più comune del copepode adulto è 1o Xiphias gladius Linn., nelle cui carni è conficcato per una parte della sua regione anteriore: dall' esterno della cute del pesce sporge di solito la porzione più resistente del copepode, costituita dall' addome e dalla porzione caudale

Guérin scrive che questa specie «habite la chair du Xiphias, du Thon etc., Méditerranée». Altri autori le assegnano come ospite 1'Orthagoriscus mola Linn. (Atlantico), avendo scambiato probabilmente una specie molto somigliante la $P$. Orthagorisci di Perceval Wright colla nostra. Il $\mathrm{D}^{\mathrm{r}}$. Baird informa che ha esaminato un esemplare di Pennella tolto da un sunfish (pesce sole) catturato a Megavissey nel Cornwall e che egli riferisce alla $P$. filosa : fu certamente il primo esemplare di tale specie di copepode trovato lungo le coste della Grande Bretagna.

Richiardi riscontrò la sua Pennella Costai parimente nelle masse muscolari dello Xiphias gladius Linn. Valle la cita per l'Adriatico e ne raccolse un unico esemplare dalle masse muscolari della regione caudale d'un giovane pesce spada pescato nel golfo di Trieste il 7 Dicembre I880.

A Genova si conservano nel Museo Zoologico della nostra Università alcuni esemplari raccolti su due di tali pesci catturati il 3 Dicembre 1880 e nel Giugno I891. Ho da notare altresi un individuo giovane di Pennella filosa inviatomi da Portoferraio, dal D.r Damiani che lo raccolse sull'opercolo di uno Xiphias gladius Linn. pescato il I6 Giugno I899, nella Tonnara Eufola. Di questo giovane esemplare femminile di copepode darò qui sotto una descrizione a parte, presentandosi con caratteri assai diversi dall' animale adulto.

Descrizione della femmina giovane. (Tav, XVIII, fig. I-7).

Il cefalotorace (fig. 4) è di forma ovale allungata e porta sul margine frontale il primo paio di antenne bi-articolate, allungate, sottili e setigere; ai lati della fronte e nel mezzo si vedono inserite due antenne uncinate cogli uncini rivolti verso l'interno (antenne del secondo paio) e coll' articolo basale piuttosto spesso. Segue un po' più in basso la bocca con apertura circolare e assai sporgente in fuori a guisa di largo e cilindrico succhiatoio (fig. 5). Essa presenta nel suo interno due piccole mandibole a guisa di bastoncini rigidi e ai lati esternamente due piccole mascelle coll'estremità bifida (fig. 7).

Un pò indietro si osservano i piedi mascellari i quali appariscono bi-articolati : l' articolo basale è spesso e verso l'esterno presenta due grosse e lunghe spinule, mentre il secondo articolo terminale è costituito da una sola lunga e forte spina, ricurva alquanto verso l'esterno (fig. 6). Non mancano poco discosti i rudimenti del secondo paio di piedi mascellari costituiti da due brevi e appuntiti uncini (piedi mascellari del secondo paio) (fig. 4).

Il cefalotorace rappresenta la porzione più larga del corpo; all'indietro si restringe gradualmente per passare al collo sottilissimo. Subito dopo il confine della regione ovale allungata detta cefalotorace, si vedono distintamente quattro paia di piedi natatori inseriti l'un paio a poca distanza dall' altro (fig. 4). Ogni singolo piede è situato lateralmente del corpo e collegato col suo rispondente dal lato opposto per mezzo di una lamina sottile chitinica mediana, ed è costituito da una lamina ovale-allungata, con due rami bi-articolati, per le due prime paia; e 
con un solo ramo bi-articolato pel terzo e pel quarto paio. Gli articoli terminali di questi rami portano brevi setole. Questa porzione del corpo che porta piedi natatori si può definire come la porzione addominale anteriore ed è divisa da tre segmenti per laterali strozzamenti.

Il corpo allungatissimo e cilindrico che va sempre più restringendosi dall'avanti all'indietro passa nell'addome mediano presentandosi per un tratto sottile quasi filiforme, ma verso 1'estremità posteriore si allarga gradualmente. E ad un certo punto cominciano ad apparire le strozzature laterali (fig. 2) che dividono il corpo in tanti segmenti e che intaccano la regione caudale. Dapprima questi segmenti o anelli arrotondati ai lati sono semplici : poco dopo appariscono muniti di espansioni laterali. Queste si presentano brevi subito dopo le prime strozzature e piegate all'indietro. Per tal modo questa regione del corpo assume nelle forme giovanili di Pennella l'aspetto di un miriapodo, i cui piedi sono come rappresentati dalle dette espansioni laterali, le quali dapprima sono semplici, ma più tardi sviluppandosi si biforcheranno e daranno a questa coda l'aspetto caratteristico di una penna, da cui il nome Pennella. La regione caudale termina con un tratto privo di appendici laterali e con due piccolissime laminette caudali con due setole per ciascuna, in mezzo a cui apresi l' orifizio anale (fig. 3). La lunghezza dell' unico esemplare da me osservato è di circa $\imath_{2} \mathrm{~mm}$.

Pennella rubra n. sp., \&, Tav. VII, fig. 3.

Descrizione della giovane femmina.

Da un Orthagoriscus mola catturato a Napoli ho raccolto due esemplari giovani di una Pennella che non mi è possibile determinare cioè non potrei, sebbene l'ospite sia identico, riferirli alla Pennella Orthagorisci di Perceval Wright, perchè di questa forma non mi è noto lo stadio larvale corrispondente. Per tale motivo descrivo siffatti esemplari col nome nuovo di Pennella rubra cosi distinguendoli per la colorazione rossa osservata negli individui freschi. Disgraziatamente questi esemplari sono guasti e incompleti. Non mi è riuscito di trarne fuori dalle carni dell' ospite la parte anteriore del loro corpo, penetrante profondamente nei tessuti muscolari del pesce: e solo ho potuto esaminare e copiare dal vero la porzione inferiore dell'addome e la regione caudale (Tav. VII, fig. 3).

Evidentemente è questa una forma giovanile di femmina. Di essa sporgeva fuori dal pesce soltanto la parte or detta del corpo, che come si vede dal disegno, mostra ben sviluppato solo l' asse primario della penna. Questa è guarnita lateralmente di numerosi rami leggermente obbliqui, rivolti all'indietro e semplici, corti, appena abbozzati, e presentansi assai differenti dai corrispnndenti rami delle Pennelle adulte, in cui tali formazioni laterali s'assottigliano, s'allungano e, alle volte, sono divise in rami secondari e danno luogo a veri ciuffi. La parte addominale e la regione del collo che stava immersa e nascosta nelle carni del pesce, per quanto ho potuto vedere, s'inoltrava assai profondamente nel tessuto adiposo e muscolare dell' Orthagoriscus, mantenendosi sottile, cilindrica, di colore giallo sporco chiaro e presso a poco sempre dello stesso diametro. La porzione cefalica avrebbe potuto da sola fornirmi gli elementi necessari per una diagnosi esatta 
di questa specie e forse rendermi certo che non si riferisca a qualche forma conosciuta, come ad es., la Pennella Orthagorisci o la $P$. fllosa che hanno per ospite il pesce sovra indicato, ma tale porzione cefalica, distaccatasi, rimase nelle carni dell'ospite nè mi riuscì di rintracciarla.

La lunghezza del corpo così incompleto, compresa la parte sporgente fuori della cute dell' ospite è di cent. $2{ }^{\mathrm{T}} / 2-3$ circa.

Habitat. - Raccolsi due esemplari sull' Orthagoriscus mola Linn. il 3 I Luglio 1903 a Napoli.

\section{Pennella Remmorae Murray $q$,}

Pennella Remorae Gerstaecker, I866-1879 (2) p. ro4.

- - CARUS, I885 (2) p. 373 .

Questa specie è citata da Gerstaecker e da Carus, pel mare Mediterraneo, ma non è detto su qual pesce. Fu trovata però, la prima volta, nel mare inglese sull'Echeneis Remora Linn., « disco cephalico inserta» (Carus).

\section{Gen. Tripaphylus RICHIARDI,}

Tripaphylus Musteli Beneden v., $q$ e $\sigma^{7}$

Lernaeonema Musteli BeNEDEN v., 185 I (2) Bull. de l'Acad. Roy. de Belgique, Tom. XVIII, p. 100, c. tav.

- - BENEDen V., I8SI (2) l'Institut, XIX, N. 922, p. 285.

- - Beneden v., I85I (3) p. I25, pl 6, fig. II, I2.

- - VogT, 1877 (2) p. 69, tav. III, fig. II.

Tripaphylus Musteli RICHIARDI, I878 (6) p. XX.

- - Richiardi, I88o (9) p. 15 I.

- - VAlle, 1880 (3) p. 70.

- - Carus, i885 (2) p. 372.

Lernqeenicus Musteli Bassetr-Smith, I899 (5) p. 485.

Distributio et habitat. - Gli esemplari femminili descritti da Van Beneden non erano completi mancando la porzione cefalica che non è stata ancora descritta Sono stati raccolti sulle branchie del Mustelus vulgaris Müll. e Henle, lungo il litorale belga. Valle trovò due soli esemplari di questo interessantissimo parassita nell'Adriatico, penetranti nei muscoli della cavità branchiale del Mustelus equestris Bp. La specie è pure citata da Richiardi pel Mediterraneo, parassita di questo pesce ora riferito, e fissata nei muscoli sul davanti della prima arcata branchiale. Van Beneden descrisse pure il maschio di questo copepode.

\section{Gen. Lernaeenicus LesueuR,}

\section{Lernaeenicus gracilis HELLER $\supsetneq$.}

Lernaeonema gracilis Heleer, I865, (li p. 249, pl. XXV, fig. 5.

Lernacenicus gracilis RICHIARDI, (4) p. 8.

$$
\begin{array}{ll}
- & -\quad \text { Valle, 1880, (3) p. } 69 . \\
- & -\quad \text { CarUs, I885, (2) p. } 372 . \\
-\quad & -\quad \text { BaSSetT-SMith, I899 (5) p. } 485 .
\end{array}
$$

Habitat. - Fu raccolta sulla pelle di Lichia amia Linn. nel mare Adriatico. 


\section{Lernaeenicus vorax RICHIARDI, ‡.}

Lerraeenicus vorax RICHIARDI, I877 (4) p. 9, pl VIl, fig. I-2I.

$$
\begin{aligned}
& -\quad-\text { Richiardi, I880 (9) p. } 150 . \\
& -\quad-\text { Valle, I880 (3) p. } 69 . \\
& -\quad-\text { Carus, I885 (2) p. } 372 . \\
& -\quad-\quad \text { BassetT-Smith, i899 (5) p. } 485 .
\end{aligned}
$$

Distributio et habitat. - Nel Mediterraneo fu trovata dal Richiardi nelle masse muscolari dell' Umbrina cirrhosa Linn., Corvina nigra Cuv. e Sciaena aquila Lac. Questo crostaceo parassita, scrive Richiardi, è comunissimo da noi; i tre quinti delle giovani ombrine sono attaccati e ne portano quasi sempre parecchi individui. Valle pure per l'Adriatico la dice specie abbastanza frequente da lui ugualmente rinvenuta sulla cavità della bocca dell' Umbrina cirrhosa Linn.

\section{Lernaeenicus neglectus RICHIARDI,.}

Lernaeenicus negleclus RICHIARDI, I877 (4) p. I3, pl. VII, fig. 22-43.

$$
\begin{aligned}
& \text { - } \quad \text { - RICHIARDI, } 1880 \text { (9) p. } 150 . \\
& \text { - } \quad \text { - VALLE, I880 (3) p. } 70 \\
& \text { - - Carus, i885 (2) p. } 372 . \\
& \text { - - BASSETT-Smith, } 1899 \text { (5) p. } 485 .
\end{aligned}
$$

Distributio et habitat. - Richiardi trovò questo crostaceo parassita molto comune su qualunque regione del corpo dei muggini (Mugil cephalus Cuv., M. capito Cuv., $M$. auratus Risso, $M$. chelo Cuv. e M. saliens Risso), fissato nelle masse muscolari, ma particolarmente verso l'estremità posteriore, a breve distanza dalla pinna caudale. Valle per l'Adriatico dice questa specie assai comune sopra qualunque regione del corpo di Mugil cephalus Cuv. e di Mugil saliens Risso, principalmente alla base della pinna anale.

Lernaeonicus sp.? q. Tav. XVIII, fig. 8-13.

Descrizione della femmina.

Il corpo è di forma allungatissima, cilindrica e nella sua parte mediana quasi filiforme (fig. 8). Sul davanti esso presenta un rigonfiamento provveduto lateralmente di due espansioni a guisa di corna; verso l'indietro si restringe e diventa sottile presentando per un buon tratto una specie di lungo collo; ma di nuovo verso la parte terminale dell'addome il suo diametro si allarga. La lunghezza è di $25 \mathrm{~mm}$. Due o tre millimetri sono occupati dal rigonfiamento cefalotoracico, otto o nove dalla parte più rigonfia, dall' addome terminale, mentre la regione più sottile del collo ne occupa la lunghezza maggiore.

Il rigonfiamento cefalotoracico (fig. 9, Io) presenta ai suoi lati due corna abbastanza lunghe, ingrossate alla base e attenuate verso l'estremità le quali sono rivolte all'esterno con leggiero cenno di ripiegamento verso l'avanti. Il margine frontale è abbastanza ampio e lascia distinguere nel mezzo una piccola prominenza: ai due lati di questa, sporgono fuori rivolte verso l'avanti, le due antenne del secondo paio bi-articolate, vistose e a forma di tenaglie. Ai lati di esse trovansi le sottili e lunghe antennule del primo paio, munite di setoline sul margine anteriore e all'estremità, e ripiegate verso l'esterno. 
Sulla parte ventrale del cefalotorace e all' innanzi del punto d'inserzione delle corna laterali si scorge una grossa prominenza quasi conica che porta gli organi boccali. Questi sono rappresentati da un succhiatoio allungato, di forma cilindrica, coll'apertura circondata da cilia. Ai lati del rostro si vedono due piccole mascelle costituite da una laminetta basale e da due prolungamenti setolosi, e più sotto originansi i vistosi piedi mascellari formati di due art oli ugualmente lunghi, quello basale più spesso, munito di una spina verso la metà di sua lunghezza, quello terminale uncinato (fig. I 2).

Al di sotto dell' origine delle due grossa corna cefaliche, sulla parte ventrale del cefalotorace e nel punto che comincia a restringersi, si vedono tre paia di piedi natatori, costituiti ciascuno da una lista mediana basale chitinica, disposta trasversalmente all' asse maggiore del corpo, che porta per ogni lato un articolo basale allungato sostenente due rami bi-articolati piccoli e setigeri (fig. I3). Tale costruzione è evidente nel primo e nel secondo paio, non così nel terzo paio dove non ho potuto discernere che l'articolo basale. Il quarto paio è mancante: al suo posto si scorge soltanto un breve tratto della lista mediana chitinica e nessuna traccia di piedi laterali.

Tutto il corpo si presenta finamente anellato o meglio striato trasversalmente sopratutto nella regione del collo. Questa è una falsa metameria tanto leggiera e fine che si rende appena visibile con forti ingrandimenti. Il post-addome nel nostro esemplare è affatto mancante.

Di tutte le specie note il solo Lernaeenicus neglectus di Richiardi, per le dimensioni e per la forma generale somiglia col nostro esemplare, però non completamente, non oso perciò ritenerlo identico, tanto più che l'esemplare da me studiato è mancante del post-addome. Alcune differenze per giunta si notano nella struttura delle rispettive membra e capo, come dal confronto delle figure di Richiardi colle nostre si rileva facilmente.

Habitat. - Fu trovato dal Prof. C. Parona un solo esemplare fissato al collo, dalla parte destra di Nettastoma melanura Rafin., a Genova il 24 Aprile Ig04.

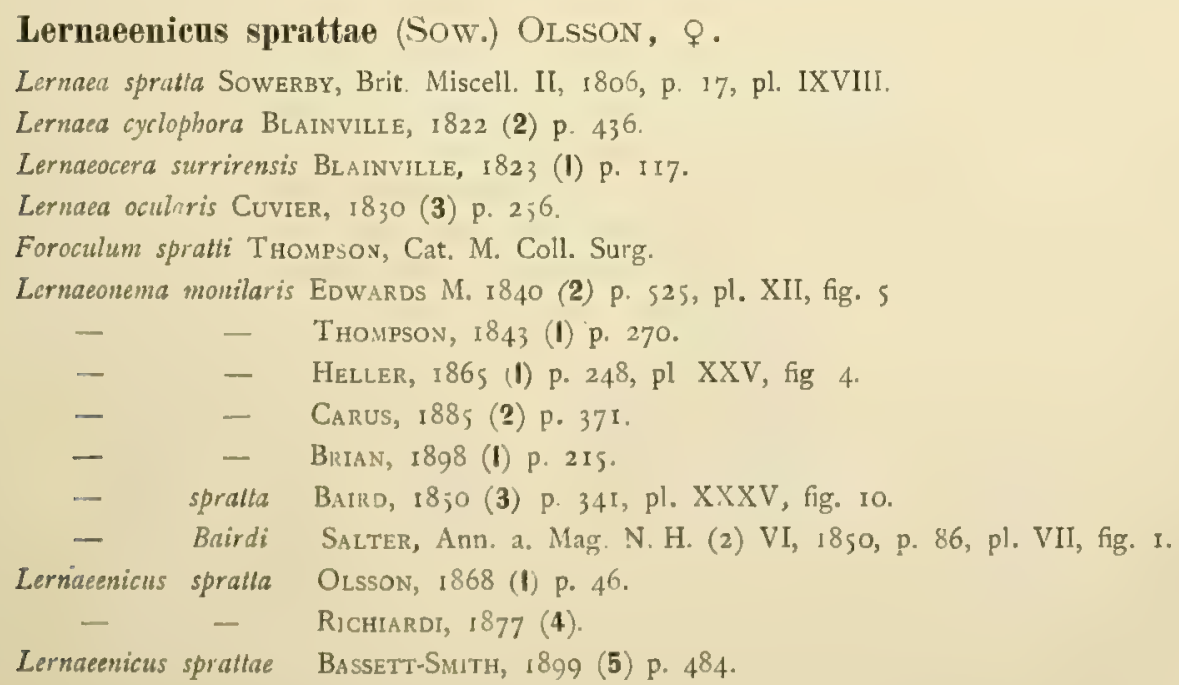


Distributio et habitat. - Heller raccolse questa specie su un occhio di una specie di Clupea nel Mediterraneo. Baird la dice trovata fissata all' occhio di una Clupea sprattus Linn. presa nel mercato di Londra dal signor W. Wing in Novembre 1848. Olsson la cita per le coste di Norvegia dove vive parassita sullo stesso pesce or nominato, fissato nella parte del corpo pure ora indicata. Anche a Genova fu raccolta aderente all'occhio della Clupea sprattus Linn. in Giugno I880.

Lernaeenicus Labracis RICHIARDI,

Lernaenicus Labracis sp. n. RICHIARDI, 1880 (9) p. I50.

Specie non ancora descritta. Richiardi la raccolse nelle masse muscolari di Labrax lupus Cuv., Box boops Linn. e Trachurus trachurus Castn.

\section{Lernaeenicus Sargi RichIARDI,}

Lernaeenicus Sargi sp. n. Richiardi, I880 (9) p. I5O.

Specie non ancora descritta. Richiardi la scoprì nella pelle del Sargus Salviani Cuv, e Val.

\section{Gen. Rebelula Poche (gen. Lophoura KöLL).}

Rebelula Edwardsii KöllikeR, ૧. Tav. XIX, fig' I. Tay. XXI, fig. 5.

Lophoura Edwardsii KöLLIKER, I853, p. 359

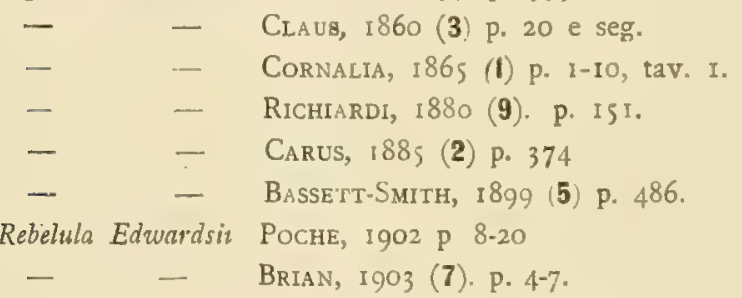

Kölliker scopriva questa forma a Messina nel 1852 sul Macrourus (Lepidoleprus) calorhynchus Risso e la denominò Lophoura Edwardsii, desumendone il nome del genere, da uno dei più salienti caratteri presentati dal parassita, di possedere cioè la coda a pennacchio. Di questa nuova specie Kölliker ci diede una breve descrizione. Piu tardi Claus aggiunse qualche cosa d'importante a ciò che conoscevasi intorno alla Lophoura nel suo lavoro sulla morfologia dei copepodi, dal quale si rileva che tanto Kölliker quando Claus studiarono un unico esemplare, il solo che fino al 1860 si fosse trovato. Cornalia nel 1865 potè studiare due altri individui di questa specie e completò la descrizione de' suoi predecessori. Gli esemplari da lui esaminati provenivano da Napoli, raccolti sul corpo di due Macrourus calorhynchus Risso; e stavano sul dorso accanto alla colonna vertebrale ed alle apofisi spinose di questa. Tutta la parte assottigliata anteriore del corpo era ficcata, al di là della pelle, nelle carni fin quasi a contatto delle vertebre. Questa parte anteriore era piegata ad angolo retto e giaceva in un canale limitato da una parete organizzata.

Distributio et habitat. - Questa forma è stata trovata sopra del Macrourus colorhynchus Risso a Messina, Napoli e a Genova, ed è stata citata per queste 
località rispettivamente da Kölliker, Cornalia e dallo scrivente. Anche Richiardi la ricorda nel suo catalogo come specie propriamente italiana.

L' unico esemplare che ho esaminato e che conservasi nel Museo Zool. della Regia Università di Genova, presentasi fissato appunto sul dorso del Macrourus colorhynchus Risso, nel modo descritto da Cornalia come negli esemplari da lui studiati. (Tav. XIX. fig. I - Tav. XXI, fig. 5). Probabilmente, per quanto si può giudicare dall'esterno, anch'esso ha la parte assottigliata anteriore del corpo penetrante al di là della pelle, nelle carni e quasi a contatto delle vertebre. Di questo parassita non si vede all' esterno che il solo addome e le paia di ciuffi di appendici cilindriche molto vistose che bene risaltano agli occhi.

Nota. - Io ritengo che il gen. Lophoura sia affine al gen. Sphyrion o Lesteira e debbasi, come quello, annoverare fra i Chondracanthidae e non fra i Lerneidae, come finora s'è fatto: Ho dato le ragioni di questa opinione in una recente pubblicazione: Brian, 1903 (7) p. 4-7.

\section{Gen. Lernaeolophus HELIER,}

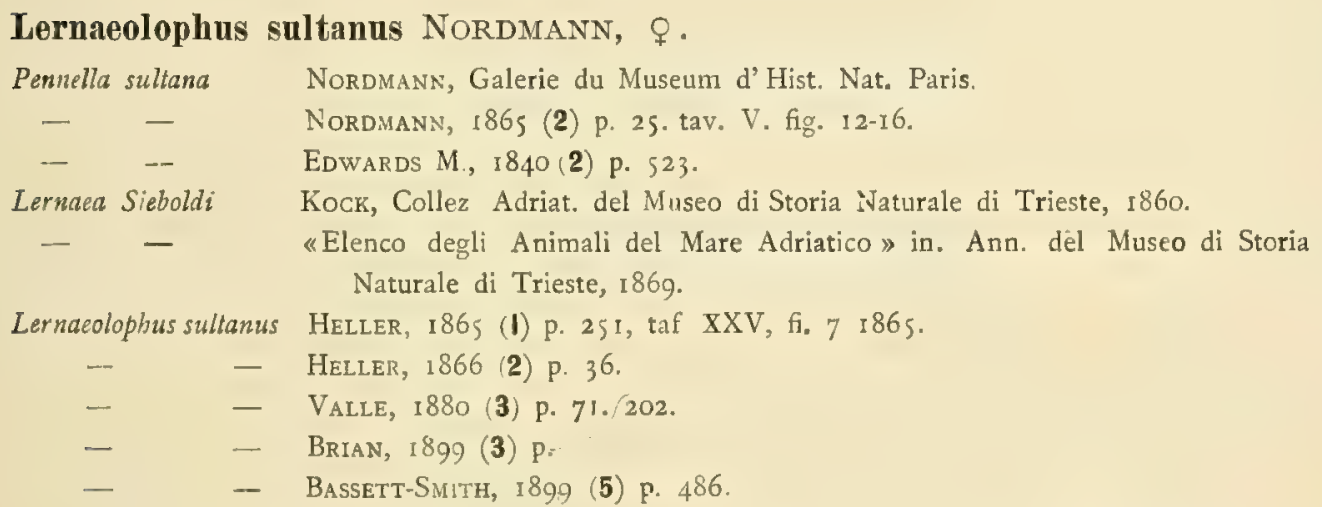

Il nome del genere fu stabilito nel I 865 da Heller il quale ne lasciò un' accurata diagnosi e descrizione. Sembra che egli non conoscesse il lavoro di Nordmann pubblicato un anno prima nel $\mathrm{r} 864$, poichè non fece alcun cenno di esso. In quello, la specie è descritta sotto il nome di Pennella sultana col quale era stata citata da Milne Edwards fin dal I 840 , perchè determinata un po' prima di quell'epoca dallo stesso Nordmann. Questi la descrisse nel 1865 in modo abbastanza diffuso e completo (salvochè per le parti boccali): ma più esatta è la descrizione di Heller.

Distributio et habitat. - Nordmann notò questa forma nella cavità boccale di Caranx ascensionis; la sua varietà sigmoidea la riscontrò sulle labbra di Scorpaena bufonia; egli ha pure trovato un esemplare difettoso nella cavità boccale di Esox brasiliensis nell' Atlantico. Heller e Valle hanno riscontrato la presenza di questa specie nelle cavità branchiali e nella bocca di Serranus scriba Cuv. e di Serranus cabrilla Cuv., ed è alquanto rara secondo Valle. I detti autori la citano pel mare Adriatico.

Ne esaminai un esemplare trovato dal D Damiani fissato alla volta palatina della Maena vulgaris Cuv. e Val. (Gennaio r899, Portoferraio). 


\section{Gen. Lernaea LINNEO,}

Lernaea branchialis LINNEO $q$ e $\sigma^{7}$.

Lernaea branchialis LinnaEus, 1767 (4) Syst. Nat. edit. 12. p. 1092.

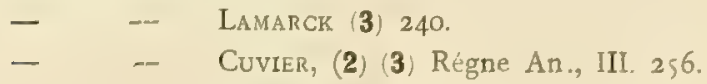

- - Burmetster, 1833 Nov. Act, XVII.

- - Guérin, 1840 f. I; Encyc. Britann., edit. 7, XXI, t. 502, t. 13.

- - KRöYer, (I) Tidsskrift, I, t. 3, f, Io (?)

- - OKen, i8is, t. 7 .

- - Edwards M, 1840 (2) pl 528.

- - Thompson, 2843 (I) p. 270.

- $\quad$ - BaIRd, 850 (3) P. 344.

- - Steenstrup et Lütken 186 I (I) p. 403, pl. Xili, fig. 28.

- - Beneden V., I8SI (3) p. 127.

- - Beneden v., 1861 (14) t. XXXIII, pl. XIX, fig. 5-12.

- - HESSE, (3) Tom. XX taf. I.

- $\quad$ - ${ }^{\prime}$ Claus, i868, (10) p. I6, pl. III, IV.

- - OlsSON, 1868 (I) p. 48 .

- - MEtzger, I868.

- $\quad$ - RICHIARDI, I880 (9) p. IsO.

- $\quad$ - CARUs, 1885 (2) p. 371

- - Thompson I. C., 1893 (2) pl. XXIV

- - MRAzeK Al, i895.

- - - SCHimkewitsch, I8g8 (3) p. 48.

-- - BASSETT-SMITH, I8g9 (5) p. 487.

- - Brian, 1902 (5) p. 34

- - Pedaschenko, I896 (1) p. 197-I99 II fig.

- - Pedaschenko 1896 (2).

- - Pedascenko, 1899 (3).

Lernaea gadina MüLIER (I) p. 65, pl. CXVIII, fig. 4 .

- $\quad$ - Fabricius O, (1) 339 .

Lernaeocera branchialis BIAINVILLE, 1822 (2) 376 , pl. XXVI. fig. I, Ia.

$\begin{array}{ll}- & \text { NoRdmann, I832 (1) p. I3O. } \\ \text { Lernaea } & -\quad \text { Ström, Phys. og Occon. Besk, t. I. f. I8. }\end{array}$

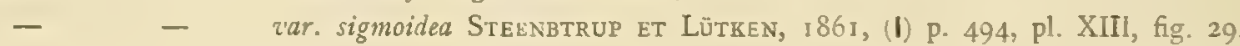

Distributio et habitat. - Fra gli ospiti di questa specie per 1'Atlantico sono da annoverarsi il Gadus morrhua Linn. (branchie); Gadus barbatus Linn. (branchie); Pleuronectes flesus Linn. (branchie).

L'ospite della Lernaea branchialis var. sigmoidea Stp. I.tk. è il Gadus ovak (branchie).

La sua distribuzione geografica è estesa dalle regioni artiche settentrionali alle temperate. Nella sua var. sigmoidea si presenta nell'Atlantico boreale al di là del $60^{\circ}$ latit., e anche la forma tipica è comune nelle regioni settentrionali, difatti Olsson scrive che questa specie abita abbastanza frequentemente i lidi di Svezia e di Norvegia sul Gadus morrhua Linn., G. aeglefinus Linn., raramente sul G. minutus Linn. (in Warberg) e $G$. merlangus Linn. (in Bahusia). Una volta egli ne notò la presenza sul Labrus myxtus Block sulla costa di Bahusia. Sul mer- 
luzzo è citata da Baird per le coste inglesi ; da Van Beneden sulle branchie di Gadus barbatus Linn. e di G. morrhua Linn. pel Belgio.

È importante la scoperta del $D^{-}$Metzger che notò la presenza delle forme giovanili (larve) tra le branchie di Pleuronectes flesus Linn. e delle forme adulte, ma giovani ancora, sulle branchie del Cyclopterus lumpus Linn. e la presenza del maschi sul Pleuronectes platessa Linn.

Mentre che questa forma di copepode sembra comune nei mari del Nord, si trova poco frequente nel nostro Mediterraneo. Richiardi la rinvenne fissata alle arcate branchiali del Merlucius esculentus Risso. Io ebbi pochi esemplari da Portoferraio raccolti dal $\mathrm{D}^{\mathrm{r}}$. Damiani sulle branchie di detto pesce.

Questa specie è l'unica fra i nostri copepodi che presenterebbe una qualche applicazione pratica. Secondo Nordmann la Lernaea branchialis adulta servirebbe di nutrimento ai Groenlandesi che la chiamano nel loro linguaggio Ovab-massimioa.

\section{Lernaea Ninnii RICHIARDI,}

Lernaen Ninnii RICHIARDi, I880 (9) p. 150.

- - C Carus, 1885 (2) p $37 \mathrm{I}$.

Specie non descritta Richiardi la trovò fissata nelle masse muscolari in prossimità dell'osso scapolare dello Smaris vulgaris Cuv. e Val.

\section{Gen. Peroderma HeLler,}

\section{Peroderma cylindricum HELLER,}

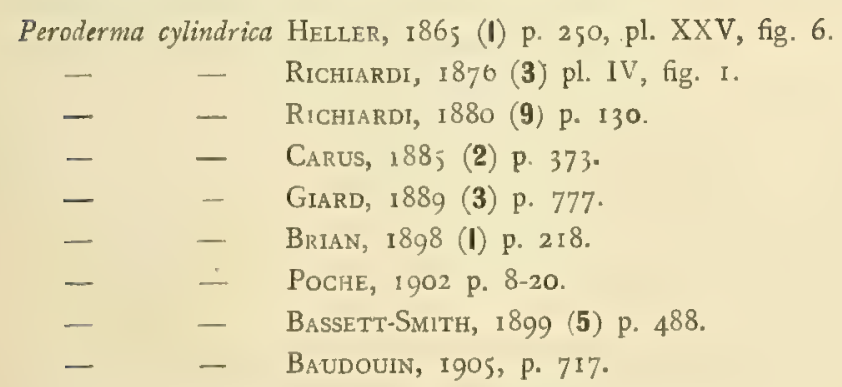

Taphrobia Pilchardi CoRnalia 1874 (2) p. I97, tav. VI.

Peroderma branchiata BassetT-Smith, I 898 (2) p. I3, pl. VII, fig. 2.

Distributio et habitat. - Heller notò questa forma parassita di una sardina pel Mediterraneo, vivente affatto nascosta nella carne del muscolo laterale. Anche Richiardi la riscontrò sull'Alosa sardina Risso, penetrante nelle masse muscolari. Io ebbi a citarla pel mare ligure.

Giard la rinvenne assai comune sulle coste di Francia a Pouliguen e a Concarneau, aderente sempre alla parte dorsale più spesso alla regione media del corpo del pesce anzidetto. Quasi sempre determina la sterilità della sardina per castrazione parassitaria; ma Giard non ha mai osservato gli accidenti di cui parla Joubin, ossia un grosso accesso che produce altre complicazioni. Come quasi tutti i parassiti a dimora fissa, scrive Giard, il Peroderma infesta la sardina giovane e s'accresce con essa, senza ritardare notevolmente l'accrescimento del pesce, ma ne impedisce lo sviluppo sessuale. 
Peroderma Bellottii Richiard, ¥. Tav. VII, fig. 2. Tav. XIX, fig. 2-5.

Peroderma Bellottii RICHIARDI, 1882. (12) p. 475-76.

- - BRIAN, I9O3 (7) P. 9.

Descrizione della femmina.

È molto facile, scrive Richiardi, distinguere il Peroderma Bellottii dal $P$. cylindricum e $P$. Petersi per i suoi caratteri specifici molto spiccati: la prima parte del corpo, costituita dal cefalotorace, e di forma ovoide, con due brevi prolungamenti verso la parte anteriore e la posteriore, che si continuano sopra l'arteria branchiale, è formata nel centro da una porzione cilindrica, sulla faccia inferiore della quale trovansi 1'apparecchio boccale e tre paia di arti, a breve distanza gli uni dagli altri, e da tutta la superficie sporgono le appendici tubulari, suddivise ciascuna in tre o quattro brevi rami: immediatamente dietro la predetta regione, dalla prima parte della porzione filiforme successiva, e seconda del corpo, sporgono come due alette, a destra e sinistra, due appendici laminari coi margini intagliati a piccoli tubercoli, il resto di questa regione sottile perfettamente cilindrica e liscia, va insensibilmente ingrossando e si continua nella terza, che forma da sè più della metà del corpo, è essa pure cilindrica, ma a superficie un poco irregolare, termina posteriormente tronca e perforata dal1' apertura anale e dalle due sessuali, alle quali aderiscono i due tubi oviferi sottili, delicati, lunghi un quarto più di tutto il corpo dell' animale.

Habitat. - Questa specie fu riscontrata da Richiardi nel Mediterraneo sullo Scopelus Benoiti Cocco, sul quale vive penetrando fra i muscoli dell'apparato ioideo. Due esemplari sporgenti fuori dell' angolo boccale dello Scopelus caudispinosus Johs. furono trovati in Genova il I8 Maggio Igor.

Fam. VI. - CHONDRACANTHIDAE.

\section{Gen. Medesicaste KRöYER,}

Medesicaste Triglarum KRöYER, $†$ e $\delta$.

Lernaea asellina LINNEO, 1746 (I) Fauna suecica, 2 I01.

- - LinNeo, (2) p. I7 I, pl. 3, fig. 4

- - LINNEO, I788 (4) p. 3145

Lernentoma asellina BLAINville, 1822 (2) p. $44 \mathrm{I}$.

- - BaIRD, 1850 (3) p. 329, tab. XXXV, fig 4.

Lerneomyzon Triglae. BLAINviLle, 1822 (2) p. 44 , pl. 26, fig. 12

- - Blainville, 1823 (I) Dict. d. Sc. Natur. XXVI, p. 125.

- - Desmarest, iS2S, p 349.

Chondracanthus Triglae NordMaNn, i $\delta_{32}$ (I) p. in6, Taf. 9, fig. I-4.

- - Guerin, i 840 , tab. IX, fig. 8

- $\quad$ - KRÖYER (I) p. I35, taf. III, fig. 3; Isis, I84I, p. 335, taf. 3, fig. 3.

- $\quad$ - Edwards M., I840 (2) p. 502.

- - Beneden V., i 85 i (3) p. rog.

Chondracanthus gurrardi Steenstrup,, Bull. Soc. Roy. Dan. i869, pl. II, fig. 3.

Oralien asellinus BASSETT-SMITH, I $\$ 99$ (5) p: 490.

- - ScotT A., 190I (I) p. 35I. 
Medesicaste Triglarum KRÖYER, 1863 (2) p. 312, tab. XVIII, fig. I.

$$
\begin{array}{lll}
- & - & \text { Heller, } 1866 \text { (2) p. } 33 . \\
- & - & \text { Richiardi, I880 (9) p. } 6 . \\
- & - & \text { Valle, I880 (3) p. } 71 . \\
- & - & \text { Brian, 1898 (1) p. 220. } \\
- & - & \text { Bassetr-Smith, 1899 (5) p. } 489 .
\end{array}
$$

Secondo Nordmann la lunghezza della femmina raggiunge senza $\dot{i}$ sacchi oviferi linee $3-3 \frac{1}{3}$, e con quelli linee $5-6$ (ossia mm. 6,54-8,70; mm. I0,90-I 3,08). Minore è la lunghezza da me osservata in esemplari raccolti a Genova; senza sacchi oviferi mm. $3 \frac{1}{2}$, con questi mm. 5 circa.

Distributio et habitat. - Questa specie si trova nell'Atlantico settentrionale (mare del Nord, Cattegat ecc.) vivente sulle branchie della Trigla hirundo B1. e della Trigla gurnardus Linn. E stata citata e descritta con nomi generici diversi (Lernaea, Lernentoma, Lerneomyzon) da Linneo, da Blainville, Desmarest, ma solo nel 1832 fu fatta conoscere in modo più completo da Nordmann che la ascrisse al genere Chondracanthus. Egli la rinvenne sulla Trigla hirundo B1. sempre internata profondamente colla sua parte anteriore nelle branchie, di modo che solo i sacchi oviferi e il margine posteriore del corpo restavano fuori.

Col nome generico sopra riferito fu notata da Van Beneden nel i 85 I sulle branchie della or detta Trigla per le coste del Belgio. Baird la citava per la fauna inglese ma sotto il nome di Lernentoma asellina (Linn.) Blain. e ne diede pure una breve descrizione con figura. Kröyer notando che questa forma ben a ragione si distacca dal genere Chondracanthus credette opportuno di sostituirle un nome nuovo e la chiamò Medesicaste Triglarum. Egli la rinvenne nella cavità boccale di Trigla hirundo B1. nel Cattegat (Danimarca). Con questo nome generico Heller la cita per l'Adriatico sulle branchie della Trigla adriatica (lineata Linn); Richiardi pel Mediterraneo sulle branchie di Trigla lyra Linn., e lo scrivente infine per il mare ligure sulle branchie di questo ultimo pesce.

\section{Gen. Strabax NordmanN.}

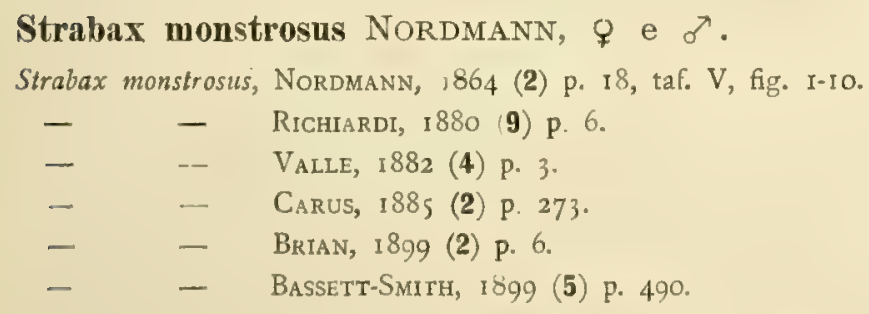

Distributio et habitat. - Nordmann ebbe 1'esemplare di Strabax monstrosus da lui studiato (I 839 ) dal prof. F. S. Leuckart di Friburgo, che 1' aveva tolto dalla lingua di una Scorpaena porcus Linn. Questo esemplare era mancante dei sacchi oviferi perchè questi probabilmente erano stati perduti nel distaccare 1'animale dall' interno della lingua del pesce, ove vive infitto e nascosto per la maggior parte del suo corpo.

Richiardi cita questa specie di copepode nel suo catalogo avendolo trovato infitto nelle arcate branchiali della Scorpaena scrofa Linn. nel Mediterraneo. Valle 
scrive che lo Strabax monstrosus è abbastanza raro nell'Adriatico: trov́ò questa forma penetrante nella lingua e nelle arcate branchiali del pesce ora menzionato, ad Umago in Istria, (Agosto I88I).

Il D." Damiani me ne inviò un esemplare da Portoferraio, da lui rinvenuto fissato nella mucosa del palato di Scorpaena scrofa Linn., il 2I Luglio I898.

\section{Gen. Chondracanthus Delaroche,}

Chondracanthus cornutus MülLeR, $§$ e $す$. Tav. VII, fig. 5.

Lernaea cornuta MüLLER, 1777 (1) p. 124, taf. XXXIII, fig. 6.

- - Mülter, Encyclop, Mèthod., Vers, tab. LXXXVIII, fig. I.

- - Linneo, 1788 (4) p. 3146.

Anops cornuta OKEN, I815, t. III.

Enlomoda cornuta LAMARCK, 1816. (3) III, p. 686.

Lernentoma cornula BLAINVILLE, I822 (2) p. 44 I.

- - Blainville, 1823 (I) p. I26.

- - BAIRD, I8jo (3) p. 328, tab. XXXV, fig. 2.

Chondracanthus cormutus Cuvier, Régne Animal IV, 258.

- - NORDManN, I832 (I) p. III, taf. IX, fig. 5-IO.

- - Edwards M., I840 (2) p. 500, pl. 40, fig. I 8-22.

- $\quad-\quad$ Beneden V., 1851 (3) p. 108, pl. 4, fig. I-4.

- - KRöYeR, I863 (2) p. 249, tab. XIII, fig. 7 .

-- - HeLLER, I 866 (2) p 33.

- $\quad$ - Beneden V., 1870 (16) pl. II, fig. I.

- - Vogt, 1877 (2) p. 76 e 8o, tab. VI. fig. 4-8.

- $\quad$ - Richiardi, 1880 (9) p. 151.

- - - VAlle, 1880 (3) P. 72.

- $\quad$ - BASSETt-SMith, I8g9 (5) p. 492.

- $\quad$ - ScotT A., IgOI (I) P. 35 I.

- - Thompson I. C. e Scott A. 1903 (2) p. 294.

(Varielas) Chondracanthus furae, KRöYER, I863 (2) p. 249, pl. XIIl, fig. 6.

» Chondracanthus soleae, KRöyeR, I837 (I) p. I29, pl. IlI.

》 - $\quad$ - Edwards M., I840 (2) p. 50 I.

\- - $\quad$ - BENEDEN V., I8jI (3) p. IOg.

Distributio et habitat. - Questa specie è stata notata ed osservata molte volte; se ne conoscono bene i due sessi. La si rinviene comunemente nell'Atlantico su diverse specie di Pleuronectes; Van Beneden la trovò parassita sul Pleuronectes platessa Linn. e Pl. Alesus Linn. lungo il litorale belga.

Heller riscontrò la presenza di questa specie nell' Adriatico sulle branchie d' un Pleuronectes. Richiardi la riporta pel Mediterraneo come parassita delle branchie della Soiea vulgaris Cuv. - Il Chondracanthus Soleae Kr. va ritenuto sinonimo col Ch. cornutus, e con questo è pure identico il Ch. furae che Kröyer descrisse nel 1863 e che egli trovò sulla Platessa limandoides B1.

Nella mia nota: Di Alcuni Crost. Par. dei Pesci dell'Isola d'Flba 1899 p. I, avevo citato il Chondracanthus pallidus v. Ben. da un esemplare femminile che così era stato determinato dal sig. I. C. Thompson, ma meglio osservato tale esemplare ha tutto l' aspetto di essere il Ch. cornutus Müller; esso fu raccolto sulle 
branchie dello Xiphias gladius Linn. a Portoferraio il 23 Gennaio I898, dal D. Damiani.

Chondracanthus Merlucii KRöYeR, 우 e $\sigma^{7}$. Tav. VII, fig. 4.

Lernaen Merhucii HoLTEN I882 pl. III, fig. 2.

Chondracanthus Merlucii KRöyer (I) I, p. 278, tab. III, fig. 9; Isis 1840 Taf. II, 3, fig. 9 a-e.

- - - Edwards M., i 840 (2) p. 503 .

-- $\quad$ - Heller, I866 (2) p. 34.

- $\quad$ - Olsson, i868 (I) p. 33.

- - - Richiardi, I880 (9) p. I5I.

- - VALLe, I880 (3) p. 73.

- $\quad$ - Carus, I885 (2) p. 354

- - Bassett-Smith, 1896 Journal. M. B. Assn. Plymouth p. 16i.

- - Schimisewitsch, W., Ig06 (I) p. 339-362.

- $\quad$ - BRIAN, 1898 (I) P. 219.

- - BASSETT - SMITH, I899 (5) p. 494.

Chondracanthus Xyphiae? Cuvier, Iconogr. Zool. pl. IX fig. 20.

Distributio et habitat. - Holten, Kröyer e Olsson citano questa specie per l'Atlantico boreale, parassita del Merlucius esculentus Risso. Anche BassettSmith rinvenne questo Chondracanthus presso le coste inglesi.

Heller scrive che si trova nell'Adriatico discretamente frequente sul Merlucius. Parimente Valle lo rinvenne abbastanza spesso in questo mare, togliendolo dalla cavità boccale di detto Merlucius esculentus Risso. Sulle branchie di tale pesce è ricordato da Richiardi pel Mediterraneo. Da Hope fu gia citato per Napoli, ed io stesso ebbi a riscontrarne la presenza in Genova, dove è stato raccolto sulle branchie di simile ospite; e di nuovo ne raccolsi un esemplare a Napoli nell' estate 1903 .

Chondracanthus horridus HeLLER,

Chondracanthus horridus HELLER, I86; (I) p. 232, pl. XXIII. fig. 4.

$$
\begin{array}{lll}
- & - & \text { Richiardi, I840 (9) p. I5i. } \\
- & - & \text { Carus, I885 (2) p. } 3.55 . \\
- & - & \text { BassetT-SMith, I899 (5) p. } 493
\end{array}
$$

Habitat. - Questa specie è citata dall' Heller e anche dal Richiardi pel Mediterraneo; essa vive sul Gobius jozo Linn.

Chondracanthus annulatus OLsson, $q$ e $\sigma$.

Chondracanthus anmulatus OLSSON, 1868 (I) p. 30 fig. 13-15.

- - Richiardi, 1882 (I2 a) p. 504-505.

Chondracanthus Laevirajae VALLE, I 880 (3) I. 73.

Descrizione della femmina.

Il corpo è allungato colla parte anteriore più angusta e inclinata. Il cefalotorace ottuso-triangolare; l'addome con due anelli distinti; la porzione genitale divisa per mezzo di una scanalatura trasversale mediana, cogli angoli posteriori prolungati in processi ottusi, brevissimi, più brevi della coda. Le antenne dell'uno e dell'altro paio sono minutissime; le membra dell' addome semplici, sub-cilin- 
driche. I sacchi oviferi molto più lunghi dell'animale. La lunghezza del corpo è di r $10-12 \mathrm{~mm}$.

Descrizione del maschio.

Il maschio è simile a quello del Ch. cornutus, tuttavia se ne distingue perchè più robusto, per la coda più allungata e curvata, pel primo paio di piedi addominali più grandi, per le antenne anteriori bi-articolate. La lunghezza del corpo è di $\mathrm{mm}$. I $=/ 5$.

Distributio et habitat. - Olsson trovò sei femmine con quattro maschi, nel mese di Agosto, fuori dei lidi di Norvegia, a Storeggen, sulle branchie di Raja batis Linn. Valle rinvenne questa forma nelle cavità branchiali d'una grande Laeviraja oxyrhynchus Bp. il 24 Febbraio nel mare Adriatico.

\section{Chondracanthus Ninnii Richrard, $q$.}

Chondracanthus Ninnii Richiard, I882 (12 a) d. IS4.

- - Richiardi, 1882 Zool, Anz. V. n. I2I p. 504.

- - VAlle, I884 (5) p. 3 .

Distributio et habitat. - Richiardi scoprì questo Chondracanthus attaccato alla mucosa della cavità branchiale del Gobius Panizzae Verga. Anche Valle rinvenne questo parassita a Venezia fissato allo stesso modo sul detto ospite, e altri esemplari riscontrò su tale pesce nella Valle di Zaule presso Trieste.

Chondracanthus angustatus HeLLER, $९$.

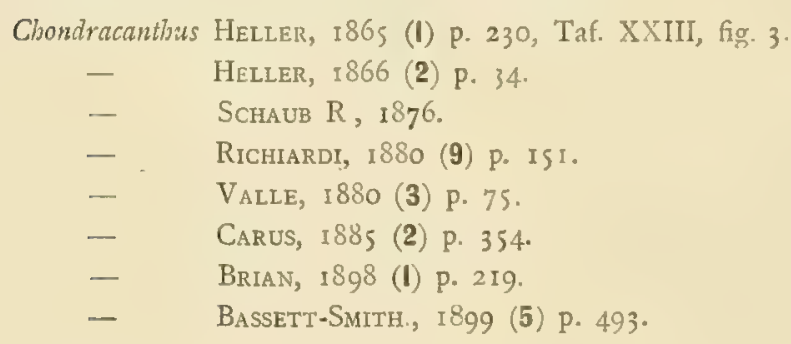

Distributio et habitat. - Heller cita questa specie parassita dell' Uranoscopus scaber Linn. pel Mediterraneo. Parimente la trovarono Heller, Valle e Schaub nell'Adriatico, abbastanza comune sulle branchie di questo pesce. Ne ho notato anch'io la presenza a Genova su detto ospite.

\section{Chondracanthus Zei Detaroche, $q$ e $\sigma^{7}$.}

I.ernacanthus Delarochiona BeAINviLLE, 1822 (2) p. 422 , fig. $1_{3}$.

- - Blainville, I823 (1) Dict. d. Sc. Natur. XXVI, p. 126.

- - Desmarest, i825, p. 350.

Chondracanthus Zei Delaroche, I 8 I I p. 270, tab. 2, fig. 2.

- - Guerrin, i 840 tab. 9. fig. 9.

- - Burmeister, I833, p. 325.

- - Lamarck, (3) p. 682.

- - Edwards M., 1840 (2) p. 504.

- - BaIrd, I850 (3) p. 327, tab. XXXV, fig. I. 
Chondracanthis Zei BeNEDEN v., I85 I (3) p. IIo, pl. 4, fig 5-7.

$$
\begin{aligned}
& \text { - - VogT, } 1877 \text { (2) p. 80, tav. V, fig. 5-8. } \\
& \text { - - Richiardi, i88o (9) p. is I. } \\
& \text { - - V VAlle, I880 (3) p. } 73 . \\
& \text { - - Bassett-Smith, i } 896 \text { Journ. M. B. Assn. Plymouth p. } 162 . \\
& \text { - - BassetT-Smith, } 1899 \text { (5) p. } 495 .
\end{aligned}
$$

Distributio et habitat. - Valle scrive che questa specie non è rara nelle cavità branchiali dello Zeus faber Linn. Richiardi la cita nel suo catalogo pel Mediterraneo presentandola come parassita sulle branchie del detto pesce.

Questo copepode è registrato per la fauna dell' Atlantico sullo stesso ospite ora nominato; Van Beneden lo ricorda per le coste del Belgio e Baird per quelle inglesi. Il primo di questi autori ha trovato siffatto parassita nel mese di Novembre e di Maggio; e nell' una e nell' altra stagione le femmine avevano i sacchi oviferi ripieni d'uova.

Chondracanthus Lophii JoHnston,

Lementoma Dufrestii Blatnville, I822 (2) p. 44I, fig. II. - $\quad$ - Blainville, I823 (I) Dict. d. Si Natur. XXVI, p i 26.

Chondracanthus Delarochianza Cuvier, 1829 (3) p. 336 , pl. I5. fig. 3.

$$
\text { - } \quad \text { - Edwards M., I840 (2) p. } 504 .
$$

Chondracanthus Lophius RIsso, 1826 (2) p. $13 \%$

Chondracanthus Lophii Johsston, I836 (4) p. 8I, fig. 16.

$$
\begin{aligned}
& \text { - - RATHKE, I843 (3) p. I16, tab. V, fig. I6-18. } \\
& \text { - } \quad-\text { Turner and Wilson, I86I-62, p. 67, pl. } 3 \text {. } \\
& \text { - - Bassett-Smith, i 896, Journ. M. B. Ass. Plymouth, p, I62. } \\
& \text { - } \quad \text { - Bassett-SMrth, 1899, (5) p. } 494 .
\end{aligned}
$$

Lernentoma Lophii BAIRD, 1850 (3) p. 330, tab. XXXV, fig. 3

Chondracanthus gibbosus KRöYER, (I) p. 252, tab. II, fig. 4; Isis I840, p. 738, taf. I12, fig, 4, taf. II 3 , fig. 2 .

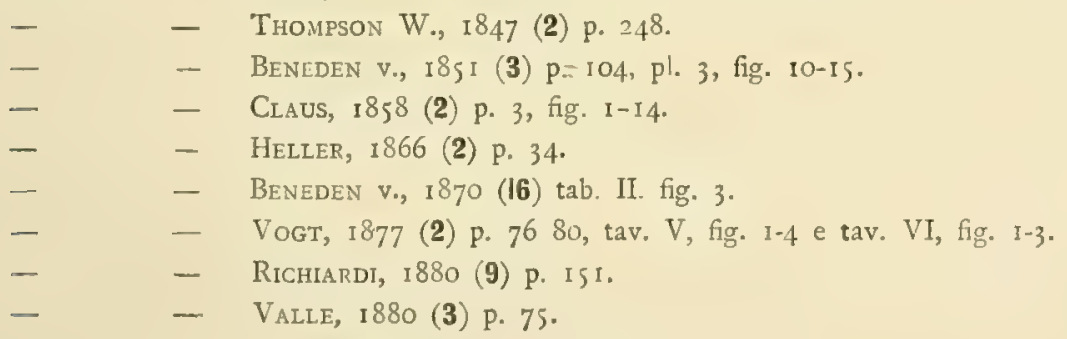

Chondracanthus gibbus $\mathrm{K}_{\mathrm{R}}$. Schimkewitsch W., 1896 (I) p. 339-362

Distributio et habitat. - $\dot{\mathrm{E}}$ specie citata da Milne Edwards come vivente sul tonno. Col nome di Lernentoma Lophii è riportata da Baird per la fauna inglese e colà trovata sul Lophius piscatorius Linn., sovra il quale pesce è ricordata da molti autori. Van Beneden riscontrò la sua presenza in gran numero di esemplari sulle branchie e sulla pelle della cavità branchiale di tale ospite, lungo il litorale del Belgio. Richiardi la rinvenne nel Mediterraneo parassita sulle branchie di Lophiz piscatorius Linn.; Heller e Valle pure 1' ascrivono per la fauna del mare Adriatico dove è comune su questo ospite. 
Fam. VII. - LERNAEOPODIDAE.

Gen. Charopinus KRöYER.

Charopinus Dalmannii RETzIUS, $q$ e $\sigma^{7}$.

Lernaea Dalmannii Rerzius, I 829 (I) p. I09, c. tav.

- $\quad$ - Retzius, 1830 (2) p. 6, fig. 5-9.

- $\quad-\quad$ Retzius, I83I (3) p. I345, tav. IX.

Lernaeopoda Dalmannii NordmanN, 1832 (I) p. 138.

- $\quad$ - KRÖYER, $1837-38$ (I) I. P. 264 , tab. II, fig. 3

- $\quad$ - KRÖYER, I840, Isis, p. 745, tab. II ${ }^{2}$, fig. II; tab. II3, fig. 4,

- - Edwards M, I840 (2) Tom. III, p si6.

- - - Turner and Wilson, 1862 I, p. 77, pl. 4.

- $\quad$ - Carus, i88s (2) p. 375

- $\quad$ - ? (Brian, I 898 (1) p. 220, tav. IV. fig. 26)

Cluaropinus Dalmannii KRöYER, I863 (2) p. 280, tav. XIV, fig. 6.

- $\quad$ - Olsson: 1868 (I) p. 4I.

- $\quad-\quad$ BENEDEN V., I870 (16) tab, II. fig. II.

- _ - VogT, 1876 (2) p. 66, tav. IV, fig. 8.

- $\quad$ - VAlLe, 1880 (3) P. 76.

-. - - BASSETT-SMith, (5) P. 498

- _ ScotT A., IgOr (I) p. 35 I.

Brachiella Dalmannii Mrculicich, Igos (2) p. 603.

? Stylophorus Hippocephalus HESSE, I879 (8) pl. 28.

Questo copepode raccolto sulla Raja batis Linn. e descritto per la prima volta da Retzius è stato citato poi da molti autori col nome di Lemaeopoda Dalmannii come lo chiamò Nordmann; più tardi per opera di Kröyer (1863) è diventato il tipo di un nuovo genere Charopinus, nel quale questo naturalista comprende una seconda specie Ch. ramosus, trovata sulle branchie di Raja clavata Linn. Anche i maschi di queste due specie sono stati studiati e disegnati dá Kröyer, I863, tav. XIV. figg. 5 e 6.

Distributio et habitat. - La prima Lernaeopoda Dalmannii scoperta dal prof. Otto e più tardi descritta e disegnata da Retzius fu trovata nelle fosse nasali di Raja batis Linn. a Christianssund. Rudolphi di poi fece avere questo stesso esemplare a Nordmann che di nuovo lo descrisse. La sua presenza su di un tale ospite è stata riscontrata più tardi da parecchi altri naturalisti. Van Beneden ha trovato diverse volte due individui in una stessa fossa nasale di Raja batis Linn., lungo le coste del Belgio. Olsson parimente trovò detta specie sull' ospite sopra riferito in Scandinavia cioè in Storeggen e nel mare di Skagerrak. Il nostro Valle rinvenne il Charopinus Dalmannii nell'Adriatico a diverse riprese; il I5 Gennaio i 880 due esemplari nelle cavità branchiali d'una Laeviraja macrorhynchus Bp., il 22 Febbraio I 880 un esemplare sulle branchie d'una Dasybatis clavata Blv., ed il 24 Febbraio I 880 sette esemplari nelle cavità branchiali d'una grande Laeviraja oxyrhynchus Bp.

In seguito a ricerche Valle crede che la specie trovata dall'Hesse nelle cavità nasali della Raja rostrata (batis Linn.) e che quegli descrisse come rappresentante d'un nuovo genere Stylophorus Hippocephalus non sia altro che il Charopinus Dalmannii di Retzius. 
Sebbene abbia creduto di adottare la denominazione di Charopinus, condivido anch'jo il parere di Miculicich nel ritenere questo genere forse identico al genere Brachiella. Desideravo bensì accertarmi con adeguato studio di comparazione del grado di affinità che si presenta tra la nostra forma sopra citata e la Brachiella malleus, con cui sembra più di tutto somigliare nella struttura (salvo che nelle dimensioni), ma pur troppo non ho mai potuto osservare alcun Charopinus Dalmannii, nè conservato in alcool nè tanto meno vivente. Se nella mia precedente nota sui copepodi parassiti spettanti alla fauna ligure ebbi occasione di citare la Lernaeopoda Dalmannii, la causa è dovuta unicamente per avere, a motivo di di questa somiglianza, scambiata la Brachiella malleus con siffatto copepode.

\section{Gen. Achtheres Nordmann,}

Achtheres Percarum Nordmann, $\subsetneq$ e $\sigma^{7}$

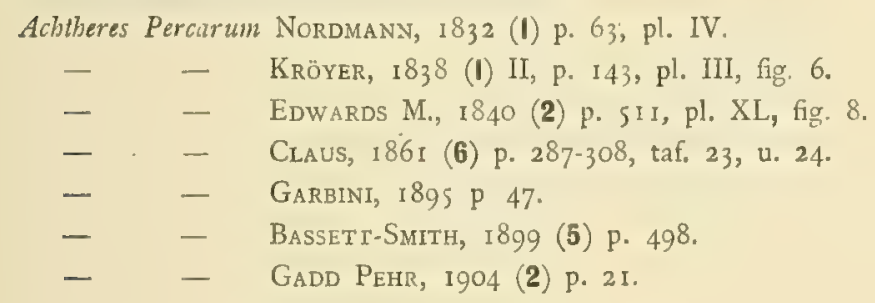

Distributio et habitat. - Vive nella cavità boccale e fissata alla lingua e al palato della Perca Auviatilis Linn., della Lucioperca sandra Cuv, e dell'Esox huius Linn., e trovasi diffusa nelle acque dolci d'Europa.

Nordmann rinvenne esemplari in tutte le stagioni dell'anno, in discreta quantità nella cavità boccale dei due pesci primi detti; molto raramente ne raccolse anche all'esterno del corpo; due volte ne trovò fissati all'occhio o meglio alla cornea. Fuori del nostro paese e sopratutto pel Nord Europa è citata da autori diversi come da Kröyer, da M. Edwards, da Claus e da Gadd Pehr, da quest'ultimo per la Finlandia. Invece per 1' Italia, per quanto io sappia, è riportata dal solo Garbini, che la rinvenne nelle acque del veronese, fissata alla gola e agli archi branchiali di una Perca.

Achtheres Galei KRöYer, $\subsetneq$.

Lernacopoda Galei KRöYER, 1837 (1) p. 272, pl. III, fig. 5.

- - Edwards M., 1840 (2) III, p. 516.

- - BAIRD, 1850 (3) p. 334, pl, XXXV, fig. 7.

- - Beneden v., I8SI (3) p. I20, pl. IV.

- - Bassits-Smith, 1896 (I) oppure Journ. M. B. Assn. Plymouth I896, p. I63.

Lernaeopoda Musteli Thompson, 1889 (2) p. 373 , pl. XXVIII, fig. 9.

Lernaeopoda Galei Richiardi, 1880 (9) p. I5I.

- - CARUS, I885 (2) p. 375.

-- - BR1AN, 1899 (3) p. 202.

- - BassetT-SMith, 1899 (5) p. 499.

Lernaeopola Scyllii RICHIARDI, I880 (9) p. ISI.

$$
\begin{aligned}
& -\quad-\quad \text { CARUS, 1885 (2) p. } 376 . \\
& -\quad-\quad \text { BRIAN, 1899 (2) p. } 6 . \\
& -\quad-\quad \text { BRIAN, 1899 (3) p. 203. }
\end{aligned}
$$


Achlheres Selachionum KuRz, I 877 (I) p. 385 , pl. XXV, fig. I.

$$
\begin{array}{lll}
- & - & \text { Valle, I880 (3) p. } 76 . \\
- & - & \text { Carus, I885 (2) p. 375. } \\
- & - & \text { BassetT-Smith, I899 (5) p. } 449 .
\end{array}
$$

Le tre specie di Lernaeopoda Galei, L. Scyllii e Achtheres Selachiorum sono, secondo me, sinonime, giacchè dal raffronto delle singole descrizioni relative a

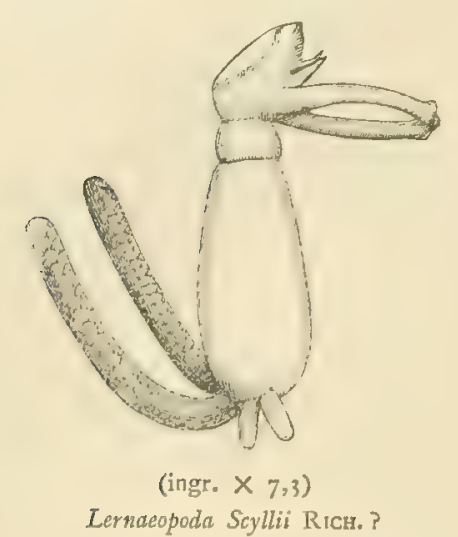
ciascuna di esse, corrispondono tra loro nei caratteri morfologici principali ( $\left.{ }^{8}\right)$. Kurz ci ha lasciato del suo Achtheres Selachiorum una descrizione molto esatta e completa. Egli assegna alla femmina di detto nome, la lunghezza di ro mm. cogli ovisacchi, di 8 a $9 \mathrm{~mm}$. senza questi. Invece per altri esemplari, quelli che precisamente sono conosciuti col nome di Lernaeopoda Galei come li chiamò Kröyer, la lunghezza totale è espressa dagli autori in 3 linee soltanto, ossia 6,54 millimetri. Anch'io ho notato in esemplari femminili raccolti a Portoferraio sullo Scyllium canicula Linn., Sc. stellare Linn. e Galeus canis Linn., una lunghezza quasi simile, variabile dai 5 ai $6 \mathrm{~mm}$., senza contare le appendici caudali lunghe da sole $1 \frac{1}{2} \mathrm{~mm}$.

I1 maschio rimase sconosciuto a Kurz e fu osservato da altri naturalisti. Secondo Vogt vive attaccato sul corpo della femmina dal lato destro o sinistro. La sua lunghezza è data da linee 2,19 ossia da mm.4,77. Secondo i disegni di Van Beneden però raggiunge appena il terzo della lunghezza della femmina. In ogni modo di lui non abbiamo ancora alcuna descrizione.

Distributio et habitat. - Il Lernaeopoda Galei fu scoperto sulla natatoria di un Galeus canis Linn. da Kröyer. Van Beneden riscontrò di poi la presenza di questo parassita non solo sulla pelle di questo pesce or detto, ma anche sulla cute di $S c y l-$ lium canicula Linn., di Mustelus vulgaris Müll. e Henle e di Trygon pastinaca Linn.

Richiardi rinvenne il L. Galei sul Mustelus equestris.Bp. ma più tardi trovò una forma di Lernaeopoda che denominò L. Scyllii, aderente alla pelle della doccia delle appendici sessuali maschili dello Scyllium stellare Linn. Avendo recentemente esaminati i Lernacopoda raccolti dal D. Damiani a Portoferraio sull'apparato genitale esterno di Scyllium canicula Linn. e di Scyllium stellare Linn. mettendoli a confronto colla Lernaeopoda Galei, ho riconosciuto la sinonimia che deve ammettersi fra queste due specie. A queste è pure identica la forma di Achtheres Selachiorum di Kurz che fu trovata da questo naturalista sul Mustelus laevis Risso e sulla Myliobatis aquila Linn. nell'Adriatico, come pure da Valle fissata all'apertura genitale del Mustelus equestris Bp. nello stesso mare.

Secondo Kurz questo copepode vivrebbe parassita soltanto sui maschi dei selacei sopra riferiti; il fatto di questa strana preferenza però non risulta ancora provato, non tutti gli autori avendo distinto il sesso degli ospiti da loro accennati per questo parassita.

(1) Kurz stesso suppose l'identità fra l'Achtheres Selachiorum e il Lernaeopoda Galei. 


\section{Gen. Brachiella Cuvier,}

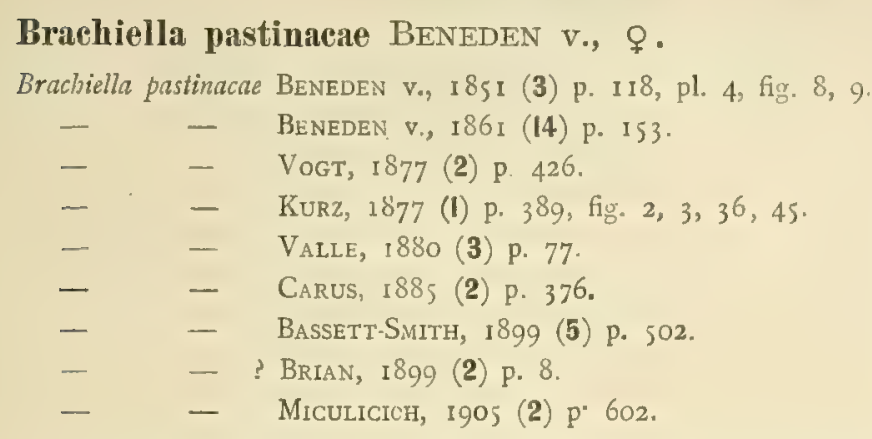

Distributio et habitat. - Van Beneden trovò questa forma nelle narici de1 Trygon pastinaca Linn. lungo le coste del Belgio. Kurz esaminò un' unica femmina senza ovisacchi raccolta nello spiracolo nasale di una Myliobatis aquila Linn. pescata a Trieste. Sullo stesso pesce e sulla Rhinoptera marginata M. H. (cavità nasali) è pure ricordata da Valle come copepode abbastanza raro nell'Adriatico.

In una delle mie note precedenti avevo citato questa specie per l' isola d'Elba, riferendomi ad un esemplare raccolto dal D.' Damiani nell' atrio della fessura branchiale di Myliobatis noctula Bp. (I I Settembre I898), ma avendo meglio esaminato questo esemplare riconobbi in lui $\mathrm{i}$ caratteri della somigliante Brachiella malleus, con cui sembra molto affine.

Brachiella malleus. RUDOLPHI, $q$ e ơ. Tav. VIII, fig. 2.

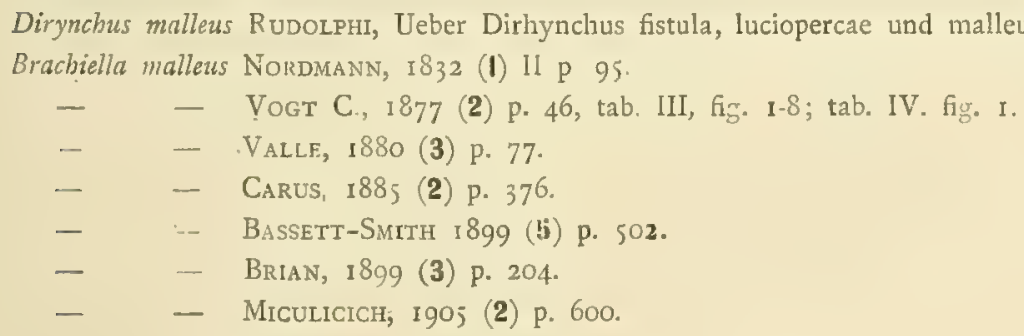

Distributio et habitat. - Questa specie fu scoperta da Rudolphi nella cavità boccale di una Torpedo marmorata Risso, a Rimini nel I8r7. Molto più tardi è stata citata da Vogt per le coste della Bretagna, da Valle per l'Adriatico, e da pochi altri autori per varie località. Di recente (I 2 Giugno I 899 ) è stata raccolta sulla mucosa boccale del sopra riferito pesce dal D.r Damiani in Portoferraio.

Io riscontrai pure la presenza della Brachiella malleus sulla Torpedo narce Nardo in Genova. L'unico esempláre quivi raccolto comparve nel mio catalogo per la Liguria, determinato sotto il nome di Lernaeopoda Dalmannii, poichè tra questa forma e la $B$. malleus esiste un rapporto di somiglianza cosi spiccato da essere tratti facilmente in inganno. La stessa somiglianza esiste colla $B$. pastinacae. Non ho ancora fatte le ricerche necessarie per accertarmi se queste tre specie ora menzionate Charopinus Dalmannii, Brachiella malleus e B. pastinacae sieno sinonime, in ogni modo fin d'ora io ritengo ciò possibile e mi riservo appena avrò il materiale sufficiente di risolvere la questione. Questa somiglianza è così accentuata che in un'altra mia nota precedente (1899) ho determinato per B. pasti- 
nacae un individuo femminile di lerneopodide, che ancor oggi sono incerto se riferire a quella specie o non piuttosto alla $B$. malleus. Con quest' ultima sembra corrispondere quasi totalmente. Tale individuo fu raccolto nell' atrio della fessura branchiale di Myliobatis noctula Bp. 1' i I Settembre I898 a Portoferraio dal D. Damiani, e presenta le seguenti dimensioni

lunghezza dell' adchome. . . . . . . . . . mm. 6

$»$ dei tubi oviferi........... . . . 3

» delle braccía . . . . . . . . . . $\$ 3$

* del cefalotoráce (dal capo all'estremità delle braccia) 》 $51 / 2$

Brachiella exigua n. sp., q. Tav. VII, fig. I. Tav, XX, fig. 7 .

Anchorella tenuis? Richiardi, I880 (9) i 52.

- - ? Carus, 1885 (2) p. 378 .

- $\quad$ - ? BRIAN, 1898 (I) p. 221.

Descrizione della femmina.

Il corpo (addome) misura $2 \mathrm{~mm}$. di lunghezza e $2 \mathrm{~mm}$. il cefalotorace colle braccia insieme comprese. L' addome ha una forma ovale allungata ma alquanto più ristretta nella regione anteriore. Termina posteriormente con un tubercolo (post-addome) che sorge sulla linea mediana a' cui lati si notano due piccole appendici all' incirca lunghe come questo. Il cefalotorace è piuttosto sottile, cilindrico, allungato e si dirige verso indietro, mentre che le braccia sono protese in avanti, riunite al loro estremo e formano quivi un tubercolo a cui è fissato un piccolo bottone chitinico a forma di coppa. (Tav. VII, fig. I).

Le antenne del primo paio sono tri-articolate e terminano con due setole. Quelle del secondo paio sono composte alla loro estremità da due rami, il ramo interno è bi-articolato, il secondo articolo è foggiato a cono e a punta. I piedi mascellari uncinati sembrano presentare oltre che un grosso artiglio allungatissimo anche un dente accessorio posto alla sua base e piccoli peli o uncinetti all' estremità del margine interno (Tav. XX, fig. 7). Forse questa specie è la Brachiella mimuta di Richiardi, che, come è noto, non fu da lui descritta ma solo nominata.

Distributio et habitat. - Raccolsi esemplari sulle branchie del Pagellus erythrimus Cuv. a Finalmarina il 27 Marzo 1897 e altri sullo stesso ospite (branchie) a Napoli nell' estate I903, ( 3 e 24 Agosto).

Brachiella insidiosa Hellek, $\&$ e $\sigma^{7}$. Tav. VIII, fig. $1,4$.

Bracbiella insidiosa Heller, I865 (1) p. 239. pl. XXIV fig. I.

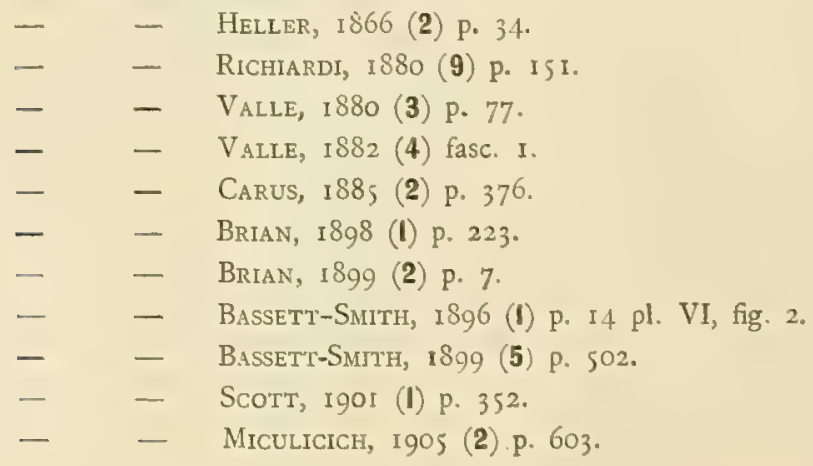


Distributio et habitat. - La Brachiella insidiosa è stata citata per 1'Adriatico la prima volta, da Heller che la scoprì sulle branchie di un Gadus sp. Richiardi poi la citava per il Mediterraneo e la trovava aderente alle laminette branchiali di Merlucius esculentus Risso. Valle pure riscontrò la presenza di tale parassita nell'Adriatico. È specie citata da Bassett-Smith per la baia di Plymouth dove fu raccolta fissata ai raggi lamellari branchiali di detto Merlucius.

L'ho citata anch' io per la Liguria e per l' isola d'Elba essendo stata trovata in queste località fissata alle branchie del Merlucius esculentus Risso. Gli esemplari da me esaminati, tutti di sesso femminile, a vero dire, non rispondono in tutto coi caratteri dati per la forma tipica dall'Heller. Una differenza si nota in essi nella diversa disposizione del cefalotorace, rispetto all' addome, il quale invece di essere rivolto in avanti, come risulta nella figura di quell'autore, è invece disposto ad angolo retto con il restante corpo od anche talora ripiegato all'indietro. Del resto 1a struttura dell'addome colle relative appendici addominali, come pure delle parti boccali è più o meno identica con quella che Heller ha descritto per la Brachiella insidiosa. Non ho creduto per questo di stabilire una specie nuova.

Uno degli esemplari provenienti da un Merlucius esculentus Risso, raccolto a Genova (quello disegnato nella tav. VIII, fig. I) presenta le seguenti dimensioni:

lunghezza totale $\mathrm{I} 2 \mathrm{~mm}$.

lunghezza dell'addome $5 \mathrm{~mm}$.

lunghezza del cefalotorace comprese le braccia $43 / 4 \mathrm{~mm}$.

lunghezza delle appendici addominali più sviluppate $4 \mathrm{~mm}$.

lunghezza dei sacchi oviferi $7 \mathrm{~mm}$.

Ho pure avuto qui a Genova due altri esemplari non completamente simili con questa specie, che ritengo tuttavia vi si riferiscano, provenienti dalle branchie di $L a-$ brax hupus Cuv. e un terzo altro abbastanza simile tolto dalla Brama Rayi B1. Schn.

Bríhiella Thynni CuṿıeR; 우 e $\sigma^{7}$. Tav. IX, fig. I.

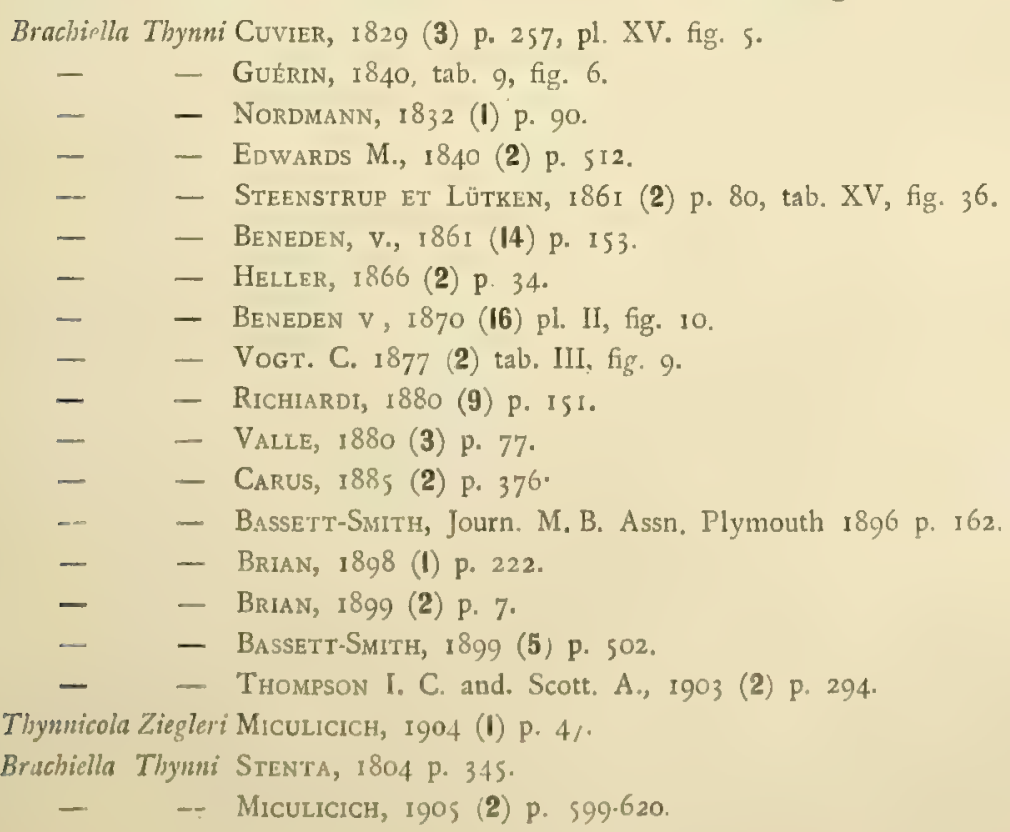


Questo animale è stato conosciuto da antichissimi tempi. A lui fanno allusione Aristotile e Plinio (Hist. Natur. IX. 2I) e più tardi Rondelet (De Insectis et Zoophytis Liber; caput VIII, de Oestro sive Asilo Marino), che però ripete quanto dai precedenti era stato detto. Questi autori non riconobbero la vera natura di questo parassita, come neppure Cuvier che ne stabilì il genere e la specie (Règne Animal III, p. 257) seppe intravedere in questo parassita la natura di crostaceo. Dopo Cuvier esso è stato citato e descritto da molti autori in modo speciale da Nordmann.

La lunghezza della femmina è, secondo M. Edwards, di ro linee circa ossia di mm. 2 I,80 e secondo Nordmann di 9 linee ossia di mm. I9,62. Io ho raccolto in Genova il 9 Gennaio 1903 un esemplare colle seguenti dimensioni:

Lunghezza totale dell'animale senza i sacchi oviferi e appendici mm. 22 circa.

Lunghezza delle braccia. . . . . . . . . . . . " 5

Lunghezza dell' addome . . . . . . . . . . " 6,5

Lunghezza degli ovisacchi . . . . . . . . . . . \ 10

Distributio et habitat. - 亡 specie comune nei mari d'Italia e parassita del tonno, sovra cui si trova, per lo più, aderente alla cute sotto l'ascella delle pinne pettorali. E una forma ben nota e citata fin dal $1846^{\circ}$ da Verany per la nostra Genova, da Hope per Nizza, e, prima, da Nordmann che ne aveva ricevuto un esemplare da Rudolphi e Schweigger, e da quest' ultimo raccolta nel Mediterraneo. Heller, Valle e Miculicich la citano per l'Adriatico, Richiardi pel Mediterrano e tanto per la Liguria come per 1'isola d'Elba la citai nelle mie note precedenti trovandola in Genova abbastanza di frequente. Al di fuori d'Italia è ricordata per le coste inglesi (Plymouth) da Bassett-Smith (che la indicò parassita anche della Sciaena aquila Lac.) e pel litorale del Belgio da Van Beneden (che la riscontrò pure sulla detta Sciaena).

\section{Brachiella neglecta RICHIARDI .}

Bracbiella neglecta Richiardi, I880 (9) p. Is T.

- $\quad$ - CARUS, 1885 (2) p. $3 \%$.

-- - Brian, I8g8 (I) p. 222 tav: IV, fig. 29.

? Brachiella sp. (Anchorella...) Brian IgO2 (5) p. 43 con fig.

Habitat. - Questa specie è stata citata dal Richiardi nel suo catalogo, come aderente al margine interno delle arcate branchiali di Sciaena aquila Lac. pel Mediterraneo, ma questo naturalista non ci ha lasciato nessuna descrizione di essa. Io trovai a Genova fra gli esemplari della collezione del nostro Museo Universitario una femmina di lerneopodide tolta dalle branchie di una Scinena aquila Lac., copepode che ritenni con dubbio, nelle mie note precedenti, potesse riferirsi alla non descritta specie del Richiardi. Ma esaminatolo di nuovo, vi ho rilevato piuttosto i caratteri esterni di una Anchorella: mi parve quindi più giusto di denominarla e di descriverla nelle pagine seguenti, come forma nuova, sotto il nome di Anchorella sciaenae n. sp: mihi. Noterò soltanto qui che somiglia all'Anchorella sciaenophila di Heller per essere munita, come quella, di quattro appendici addominali di lunghezza uguale, ma ne differisce per la presenza in più di 
un' altra appendice mediana (post-addome) frapposta ai sacchi oviferi. Misura una lunghezza da 8 a ro $\mathrm{mm}$. circa.

Brachiella impudica NordmanN, $q$ e $\sigma^{7}$. Tav. VIII, fig. 6 .

$$
\begin{aligned}
& \text { Bracliella impudica NoRDMANN, I832 (I) p. 92, taf. VIII, fig. I-3. } \\
& \text {-. - EDwards M., I840 (2) P. } 513 \\
& \text { - - Heller, I866 (2) p. } 35 . \\
& \text { - - - VAlle, I880 (3) p. } 78 . \\
& \text { - - C Carus, i885 (2) p. } 376 . \\
& \text { - - Brian, IgO3 (9) p 8I. } \\
& \text { - } \quad \text { - Miculicich, 190; (2) p. } 602 .
\end{aligned}
$$

Distributio et habitat - Questa specie fu raccolta, per la prima volta, dal D. Mehlis sul Gadrus aeglefinus Linn., ignoriamo in quale località, e fu descritta da Nordmann. Indi venne riscontrata dall' Heller sulle branchie di Trigla corax Bp. nell'Adriatico; sulla Trigla lineata Linn., e T. corax Bp. da Valle nello stesso mare. Non risultava che essa fosse stata ancora trovata pel Mediterraneo, quando recentemente il D.r Damiani ne raccolse due esemplari femminili e uno maschile sulle branchie di Trigla lineata Linn. a Marciana marina, (I6 Dicembre I9o2), altri sulla Trigla corax Bp. e T. lineata Linn. il 24 Marzo 1904 e il I4 Gennaio I905 a Portoferraio.

Brachiella Merlucii Bassett-Smith, \& e $\sigma^{7}$. Tav. VIII, fig. 3. Bracbiella Merlucii Bassetr-Smith, I8g6 (t) p. I4 pl. VI fig. 1.

$$
\begin{aligned}
& \text { - } \quad \text { Bassetr-Smith, I899 (5) p. } 503 . \\
& \text { - } \quad \text { Thompson I. C. and ScotT. A., } 1903 \text { (2) p. } 294 .
\end{aligned}
$$

Distributio et habitat. - Questa specie fu citata e descritta per la prima volta da Bassett-Smith per le coste inglesi. Egli trovò questi parassiti sempre attaccati alle punte delle arcate branchiali del Merlucius esculentus Risso e non attaccati alle lamine branchiali del medesimo come la $B$. insidiosa.

Anch'io ne ho osservato varii esemplari raccolti in Italia (Genova e Portoferraio, Napoli) parassiti delle branchie del detto pesce. Mi sembra una forma piuttosto frequente. A Napoli fu da me raccolta il 26 Agosto 1903.

L'esemplare che figura disegnato a Tav. VIII, fig. 3, è stato raccolto a Genova.

$$
\begin{aligned}
& \text { Brachiella ramosa RichIARDI, ‡. } \\
& \text { Bracbiella ramosa Richiardi, is\%o (9) p. Is T. } \\
& \text { - - Richiardi, I880 (8) p. } 69 \text { N. } 48 . \\
& \text { - - CARUS, I885 (2) p. } 376 . \\
& \text { - } \quad \text { - BRIAN, IgO2 (7) p. IO. } \\
& \text { - } \quad \text { - Miculicich, } \operatorname{rgos} \text { (2) p.603. }
\end{aligned}
$$

Descrizione della femmina.

Questa Brachiella si distingue dalle altre specie finora descritte, principalmente per la forma molto ramosa di appendici delle cosidette braccia, o piedi mascellari del secondo paio, e dei due lobi laterali terminali dell'addome; per questi caratteri Richiardi la chiamò Brachiella ramosa. 
Habitat. - Essa si trova fissata agli archi branchiali dello Xiphias gladius Linn. È citata dal Richiardi pel Mediterraneo. Io ne rinvenni un solo esemplare femminile fissato alle branchie dello stesso pesce, il 7 Maggio rgoo, a Genova.

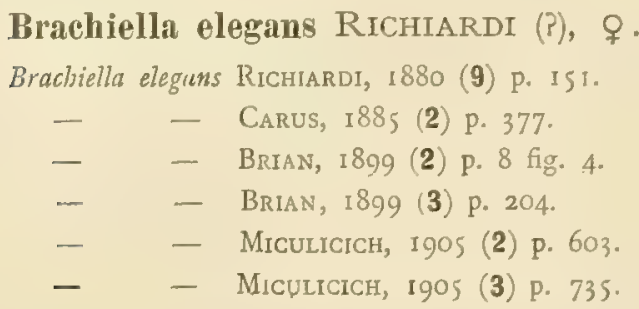

Habitat. - Specie non descritta dal Richiardi. Egli la trovò aderente al margine interno delle arcate branchiali della Lichia glauca Linn. Io ho riferito, con dubbio, nelle mie note sopra citate, a questa specie, alcuni esemplari di Brachiella avuti da Portoferraio e raccolti dal D.r Damiani, il I9 Giugno 1898 , sulle pieghe della mucosa branchiale della Lichia amia Linn. e un'altra volta sulla mucosa intermascellare e sul cavo branchiale dello stesso pesce (Maggio 1899). Questa forma somiglia alla B. Thynni e forse non ne è che una varietà (Miculicich).

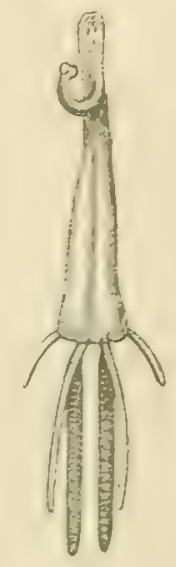

(ingr. $\times 3,6$ )

Bracbiella sp. (elegans Rich. ह)

\section{Brachiella inconcinna RICHIARDI,}

$$
\begin{array}{ccl}
\text { Brachiella inconcinna } & \text { RichIARd, } 1880 \text { (9) p. I5I. } \\
- & - & \text { CARUS, I88s (2) p. } 377 . \\
-- & - & \text { Miculicich, I905 (2) p. } 603 .
\end{array}
$$

Specie non descritta. Richiardi la trovò aderente alla mucosa del pavimento della bocca e davanti della prima fessura branchiale della Raja maculata Mont. Mediterraneo.

\section{Brachiella minuta RICHIARDI,}

Bracbiella minuta RichIARDI, I880 (9) p. I 1 1.

$$
\begin{aligned}
& \text { - } \quad \text { Carus, i885 (2) p. } 377 . \\
& \text { - } \quad \text { Miculicich, igos (2) p. } 603 .
\end{aligned}
$$

Specie non descritta. Richiardi la osservò aderente alle laminette branchiali del Pagellus erythrimus Cuv. Mediterraneo.

\section{Brachiella obesa RICHIARDI, \\ Brachiella obesa RICHIARDI, I88o (9) p. ISI. \\ - $\quad$ - Carus, 1885 (2) p. 377. \\ - - Micllicich, igos (2) p. 603.}

Specie non descritta. Vive aderente al margine interno delle arcate branchiali della Trigla corax Bp. Mediterraneo.

\section{Brachiella oblonga VALLE,}

Brachiella oblonga VALIE, i 880 (3) p. 76.

- - Miculicich, igo5 (2) p. 603. 
Habitat. - Valle ha trovato questa specie abbastanza comune sotto le pinne pettorali del Mugil cephalus Cuv. e Mugil saliens Risso. L'autore non ne ha dato però alcuna descrizione nè disegno. Adriatico.

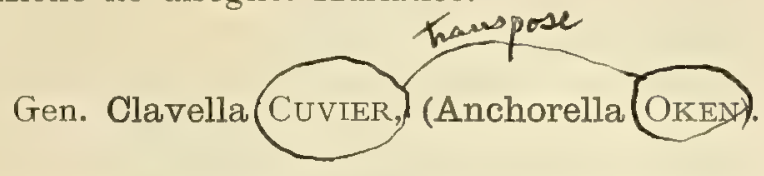

Divisione A - Post-addome mancante o appena distinto.

Clavella emarginata KRöYER, $q$ e $\sigma$. Tav. X, fig. 3 .

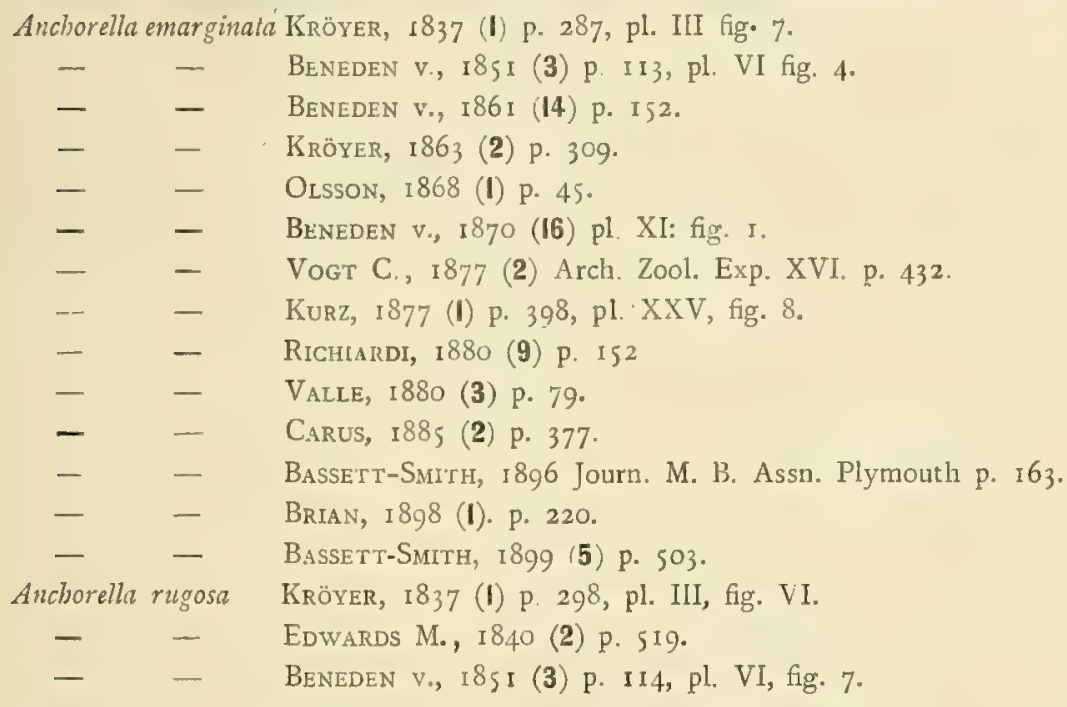

Distributio et habitat. - Milne Edwards attribuisce alla Clavella emargmata Kröy. come ospite 1'Alosa finta Cuv. e alla Cl. rugosa Kröy. l'Anarrhicas lupus Linn. Van Beneden trovò la prima di queste Clavelle sulle branchie dell'Alosa finta Cuv. e dell'Alosa communis Cuv. (coste del Belgio) e la seconda parimente sul1'Anarrhicas lupus Linn. La Clavella rugosa, secondo Baird, in Inghilterra, avrebbe anche per ospite il Gadus callarias (giovane di Gadus morrhua Linn.) Olsson raccolse due esemplari di questa specie sulle branchie di Anarrhicas lupus Linn. in Warberg e nello stretto di Öresund (Scandinavia). Egli comprende pure 1'Anchorella emarginata Kr. nella fauna della Scandirravia, avendo esaminati sei esemplari di essa raccolti dalle branchie del sopra nominato Anarrhicas, nello stretto di Öresund; e altri, nel Mus. Lund., provenienti da un tale ospite.

Carus riassumendo i dati sull' habitat di questa specie (da lui indicata col1'ultimo nome) pell'Adriatico e pel mare germanico enumera altri ospiti Alosa pontica Eichw., Scorpaena porcus Linn., Atherina hepsetus Linn. e la riferisce inoltre anchẻ alla fauna del mar Nero.

In Italia fu riscontrata tanto pel Mediterraneo come pell'Adriatico parassita sugli archi branchiali dell'Alosa vulgaris Val. Io la notai a Genova sulla Chupea finta Cuv. e non già sul Pagellus centrodontus Cuv. e Val. come per sbaglio di trascrizione, fu stampato nel mio catalogo di cop. par. dei pesci della Liguria, 1898. 
Divisione $\mathrm{B}$ - Post-addome bene distinto: $\alpha$. senza appendici addominali posteriori.

Clavella uncinata MüLLER, $q$ e $\delta$.

Lern.ter uncinata MüLLER, 1777 (1) p. 120, tab. 33, fig. 2. Anche nell'Encyclop. Méthod., Vers. tab. 78 , fig. 7 .

- - L Linneo, I788 Systema Naturae, XIII édit. Tom. VI, Vermes, p. 3I45.

- - Johnston, 1835 (2) p. 565, fig. 53.

- - Lamarck, (3) p. 684.

- - Thompson W., 1843 (I) p. 270.

Schisturus uncinatus OKEN, I8IS p. I83.

Clavella uncinata OKEN, 1815.

Anchorella lagenuia Cuvier, Icon. du Règne Anim. pl. 9, fig. 5.

Lernaeomyzon antinalum BLAinvilLe, 1822 (2) p. 438; 1823 (I) p. 122.

Ancisorella uncinata NordmanN, 1832 (I) p. 102, taf. VIII, fig.8-12. Taf. X, fig. I-5.

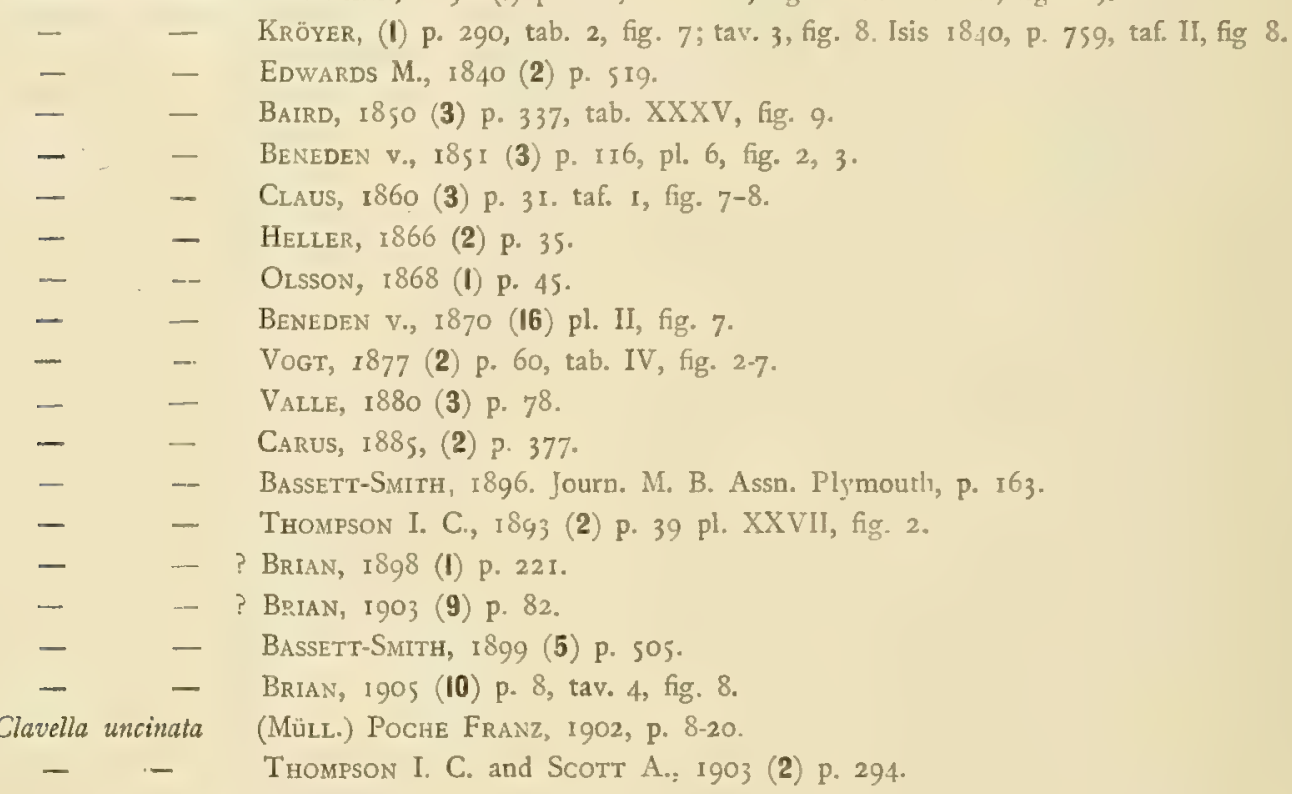

Distributio et habitat. - La Clavella uncinata vive su diversi merluzzi: più particolarmente è notata come parassita del Gadus morrmu Linn. e del G. barbatus Linn., come pure del Merlangus vulgaris Linn. Secondo Johnston vive fissata alle pinne e agli opercoli branchiali, anche alle branchie del merluzzo ed è probabilmente la specie più comune dell'oceano lungo le coste dell' Europa del Nord. Degli esemplari studiati da Nordmann parte erano stati raccolti sulle branchie di Gadus aeglefmnus Linn., parte sulla lingua del Blennius gunellus Linn.

Van Beneden nella sua nota pubblicazione «Les Poissons des Côtes de Belgique» a tav. II. fig. 7, disegnò una Clavella uncinata femmina, specie che,

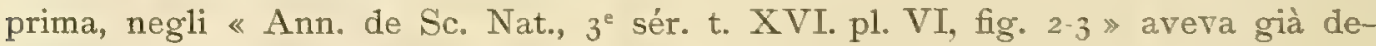
scritto ed illustrato, e che aveva trovato sul Gadus aeglefinus Linn.

Olsson per la fauna scandinava citò questo parassita raccolto frequentemente sulle branchie e specialmente (in fauce) nella bocca di Gadus morrhua Linn. (in località di Warberg, Bergen, Aalesund); sulle branchie di Gadus virens Linn. (a Skagerrak) e di Gadus aeglefinus Linn. (a Bergen). 
Heller per l'Adriatico lo ricorda sulle branchie di Merlucius sp. Per la Liguria e per l'isola d'Elba avevo notata la presenza di questa specie sui Sargus ne' miei opuscoli precedenti. Ma ulteriorj osservazioni mi hanno fatto dubitare sull' esattezza della mia prima determinazione e m'indussero a ritenere che si tratti di una nuova specie, simile alla Clavella centrodonti V. Ben. Soltanto nel materiale raccolto da Costa, conservato nel $\mathrm{R}$. Museo Zoologico napoletano, mi fu dato di esaminare un bel esemplare con tutta certezza di $\mathrm{Cl}$. uncinata, lungo $6 \mathrm{~mm}$. (coi sacchi oviferi $\mathrm{I} 3 \mathrm{~mm}$.) che ho disegnato nella tavola della mia pubblicazione riguardante quel materiale. L'ospite non era indicato ed ignoro se questo esemplare fosse stato raccolto propriamente a Napoli.

Clavella Pagri Kröyer? $९$. Tav. X, fig. 5 .

$$
\begin{aligned}
& \text { Anchorella Pagri Kröyer, I } 863 \text { (2) p. } 301 \text { pl. XVI. fig. } 9 . \\
& \text { - - Vogt, } 1877 \text { (2) (in Arch. Zool. Exp. p. 432). } \\
& \text { - } \quad \text { - RichIARDI, } 1880 \text { (9) p. } 152 . \\
& \text { - - Carus, } 1885 \text { (2) p. } 377 . \\
& \text { - -. ?Brian, } 1898 \text { (1) p. } 222 . \\
& \text { - - BASSETT-SMith, I899 (5) p. } 505 .
\end{aligned}
$$

Descrizione della femmina.

Il capo è piccolo (contenuto cinque volte presso a poco nella lunghezza del collo) poco distinto da questo: collo allungato, più largo alla base, gracile nella sua parte anteriore, rugoso; le braccia sono allungate, più lunghe del doppio che il capo), alla base non del tutto saldate, coll' apice che si estende in un disco carnoso, che quasi del tutto nasconde un bottone piccolissimo, appena petiolato, anteriormente convesso ; addome ovale, anteriormente acuminato, mostrando posteriormente tre piccoli tubercoli, quasi a mala pena della stessa grandezza: secondo Kröyer il tubercolo di mezzo sarebbe il post-addome; a quelli laterali stanno affissi i sacci oviferi esterni. La lunghezza della femmina è secondo il detto autore di due linee $(4,36 \mathrm{~mm}$.). Le parti boccali secondo le mie osservazioni hanno somiglianza notevole di struttura con quelle della Clav. Sargi, con questa differenza che gli articoli basali dei piedi mascellari del secondo paio sono avvicinati fra loro alla base mentre nella $C l$. Sargi sono alquanto più distaccati. Gli esemplari da me esaminati presentavano una lunghezza totale da $3 \mathrm{~mm}$. e $3 \frac{3}{4} \mathrm{~mm}$. coi sacchi oviferi. In un esemplare la lunghezza del cefalotorace è di $2 \mathrm{~mm}$; quella dell'addome di $2 \mathrm{~mm}$; e quella dei sacchi oviferi parimente di $2 \mathrm{~mm}$. I sacchi oviferi in alcuni individui sono piuttosto ovali e massicci; in altri più allungati e cilindrici.

Distributio et habitat. - Gli esemplari studiati da Kröyer furono raccolti sul Pagrus vulgaris Cuv, e Val. nel Mediterraneo. Richiardi rinvenne la Clavella Pagri Kr., non solo su di questo pesce, aderente alla arcate branchiali, ma anche sul Pagellus erythrinus Cuv. Questa forma è stata anche citata da me per la Liguria, parassita del Pagellus erythrinus Cuv. e del Pagrus vulgaris Cuv e Val.

Clavella Pagelli KRöYer ? $\subsetneq$.

Anchorella Pagelli Kröyer, 1863 (2) p. 275. tab. XVI, fig. 3.

- - Heller, I866 (2) p. 35. 


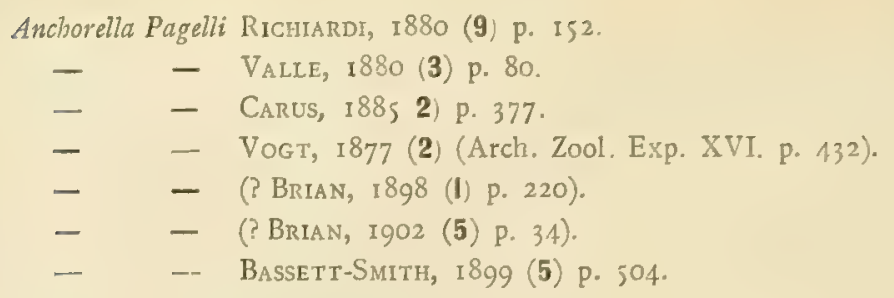

Descrizione della femmina.

Il capo piccolo, pochissimo acuminato, non distinto dal collo; collo lungo, piuttosto largo; braccia abbastanza lunghe, terminanti non già con un bottone ma con una clava cornea allungata; anello genitale ovale, posteriormente con due piccoli tubercoli, tra cui s'avanza 1'addome minutissimo.

La lunghezza della femmina è secondo Kröyer di una linea e mezzo (4,08 mm.).

Distributio et habitat. - Da Kröyer questa specie è stata indicata come parassita di un Pagellus del Mediterraneo. Heller per 1'Adriatico lá riscontrò parassita sulle branchie di Pagellus erythrinus Cuv. Richiardi pel Mediterraneo la ricorda nel suo catalogo, aderente alle arcate branchiali del Pagellus mormyrus Cuv. Valle dice che questa specie di Clavella trovasi nell' Adriatico aderente alle arcate branchiali del Pagellus erythrinus Cuv. e del $P$. mormyrus Cuv., ma è alquanto rara. Io avevo riferito a questa specie, però con dubbio, alcuni esemplari raccolti a Genova sulle branchie del Pagellus mormyrus Cuv. e del P. exythrinus Cuv., e altri trovati dal $D^{r}$. Damiani sulle branchie di quest' ultimo pesce a Portoferraio. Questi esemplari tho non somigliano alla figura data da Kröyer; avendoli di nuovo esaminati, recentemente ho stabilito per essi il nome nuovo: $\mathrm{Cl}$. strumosa, specie qui sotto descritta.

Clavella strumosa n. sp. ९. Tav. IX, fig. 4-7 - Tav. XX, fig. 8-I2. Anchorella pagelli $\mathrm{K}_{\mathrm{R}}$ ? Brian, I8g8 (I) p. 220.

$$
\text { - - ? BRIAN, I9O2 (5) p. } 34 .
$$

Descrizione della femmina.

È una Clavella con capo bene distinto dal collo. Questo va ingrossandosi verso l' addome. In alcuni individui, alla base del collo si, vede un ingrossamento sacciforme che dà alla specie un aspetto caratteristico, (da ciò l'aggettivo specifico di strumosa = gozzuta). Tale ingrossamento non è simmetrico; si trova in generale da una sola parte del collo e corrisponde con piccolo tubercolo rotondo, dall' altra. Due tubercoli però simmetricamente disposti, uguali e meno visibili si presentano in altri esemplari, forse giovanili. L'addome è ovale, ristretto in avanti. Il post-addome è piccolo e a un dipresso, simile a quello della Clav. fallax. Spesso porta attaccati i due spermatofori.

In molti esemplari il collo è proteso dritto verso l'avanti e non forma angolo nel suo punto $d^{\prime}$ 'origine coll' addome, mentre le braccia sono dirette all' indietro. Altri pochi individui presentano il collo disteso più verso un lato, e allora possono non presentare caratteristiche proprie alla specie e facilmente possono essere scambiati per Clavella Sargi o per Cl. fallax, a cui realmente somigliano; ma dalla prima di queste specie la nostra forma si distingue sempre per il post-addome 
che non è molto prominente, che è piccolo e sorge dal lato posteriore del corpo, in mezzo ad altre due laterali prominenze a cui stanno affissi i sacchi oviferi: dalla $\mathrm{Cl}$. fallax si riconosce subito per avere 1'addome più ovale, più assottigliato in avanti, e per la forma generale del suo corpo non tozza, ma più svelta.

Le antenne del secondo paio presentano di solito verso l'estremità libera quattro piccolissimi tubercoli a punta (Tav. XX, fig. 8). Le altre estremità boccali somigliano a un dipresso con quelle della Clavella Sargi. (Tav. XX, fig. 9-II). Il margine interno dell' articolo uncinato del secondo paio di piedi mascellari presenta in più una dentellatura molto fine, la quale facilmente sfugge alla vista. Le braccia sono abbastanza lunghe e il bottone chitinico è piccolo, brevemente petiolato, e inserito sopra un disco carnoso. (Tav. XX, fig. I2). I sacchi oviferi sono discretamēnte lunghi.

La lunghezza totale del corpo, compresi i sacchi oviferi, varia da 5 a 6 millimetri. In alcuni esemplari il corpo misura mm. $3 \mathrm{~T} / 2$ di lunghezza e i sacchi oviferi mm. $2 \mathrm{~T} / 2$.

Distributio et habitat. - Fu raccolta sulle branchie di Pagellus erythrinus Cuv. nel Mediterraneo (Portoferraio e Genova).

Nell' estate $1903 \mathrm{mi}$ fu dato di trovare due esemplari femmine che somigliano alquanto ma non completamente a questa specie, sulle arcate branchiali di Box salpa Linn. a Napoli, e che quindi non sono certo se si possano riferire ad essa, potendo invece costituire una n. sp. Dei due individui uno appariva diverso nella forma dall'altro, per la disposizione del collo, e anche perchè mancava dell' ingrossamento sacciforme impari älla base del collo, notevolissimo soltanto nell' altro esemplare, anzi di uno sviluppo affatto straordinario, come non avevo mai visto in nessuno degli esemplari trovati sopra il Pagellus. Quest' ultimo individuo è stato disegnato da me a Tav. III, fig. 6.

Clavella Sargi Kurz, $\subsetneq$ e $\sigma^{7}$. Tav. IX fig. 2. Anchorella Sargi KuRz, I877 (I) p. 393, fig. 5, 6, 29, 51, 52.

- $\quad-$ Richiardi, I880 (9) p. I\$2.

- - V V Vlle, i880 (3) p. 80.

- - Carus, i885 (2) p. 378 .

- $\quad$ - BRtan, 1898 (I) p. 222.

- - BAsSetT-SMIth, 1899 (5) p. 504.

Distributio et habitat. - Kurz trovò la Clavella Sargi $q$ e $\sigma^{7}$ a Trieste sulle branchie di Sargus annularis Linn. Richiardi riscontrò questa specie aderente alle arcate branchiali di Sargus Salviani Cuv. Val. e di S. annularis Linn. pel Mediterraneo. Valle scrive che è forma frequente nell'Adriatico, sulle arcate branchiali del Sargus annularis Linn. Come si vede dalle mie note precedenti anch' io ho citata questa specie in Genova sulle branchie di quest' ultimo pesce. Ne rinvenni un esemplare a Napoli fissato alle arcate branchiali di Sargus vulgaris Groeff., il 28 Luglio rgo3.

Clavella Characis Richtard, $\nmid$. Tav. X, fig. I.

Anchorella Characis Richiardr, 1880 (9) p. 152

- $\quad$ - Carus, 1885 (2) p. 378 .

- - Brian, 1899 (2) p. 9. 
Descrizione della femmina.

Il collo è allungato e alquanto allargato all' estremità libera e con ringonfiamento verso il punto di congiunzione coll'addome il quale è voluminoso quasi sferico, oltremodo rigonfio, e terminato dal lato posteriore con un piccolo postaddome rudimentale in mezzo ai due vistosi sacchi oviferi. Esternamente questa specie somiglia molto alla Clavella Sargi ma è alquanto più grossa di quella.

Il primo paio di piedi mascellari è situato sul confine posteriore del cefalotorace ed è rivolto all' indietro in direzione della parte terminale dell' addome. Questi piedi sono corti e fusi in un apparato impari di fissazione, soltanto i fasci muscolari pari lasciano riconoscere distintamente la loro origine doppia. Si notano due grosse papille verso la base di questi piedi appunto come si osservano nella Clavella Sargi e nella Cl. fallax. È una specie questa assai grossa perchè misura la lunghezza di $6 \mathrm{~mm}$. Del resto la struttura delle parti boccali non si discosta molto da quella della $\mathrm{Cl}$. fallax.

Fu raccolta due volte dal D. Damiani a Portoferraio aderente alle branchie di Charax puntazzo Linn. il 23 Agosto e I Febbraio 1903. Essa fu citata da Richiardi pel Mediterraneo su questo ospite, ma non descritta.

Clavella fallax Heller, $Q$ e $\sigma$. Tav. X, fig. 4 .

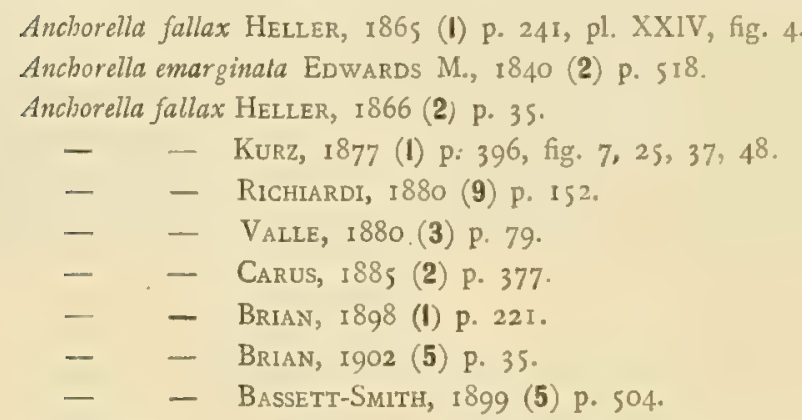

Distributio et habitat, - Heller pel Mediterraneo e per l'Adriatico notò questa specie of e , sulle branchie del Dentex vulgaris Cuv. Kurz parimente la cita per l'Adriatico e come parassita dello stesso pesce. Anche Richiardi elenca nel suo catalogo questa forma da lui riscontrata aderente alle arcate branchiali del medesimo ospite pel Mediterraneo. Valle scrive che questo copepode si trova comune nell'Adriatico. Ne riscontrai la presenza tanto presso le coste della Liguria quanto presso quelle dell' isola d'Elba e nelle acque del golfo di Napoli sempre sopra del Dentex vulgaris Cuv.

Clavella alata n. sp. \&. Tav. III, fig. 5. Tav. XX, fig. 5-6.

Descrizione della femmina.

È questa una forma che io ritengo nuova. Si distingue dalle altre Clavelle per la presenza di due sviluppate alette (che è vero si riscontrano pure nella (lavella fallax e in altre specie, ma che qui si presentano più vistose del solito) situate alla base delle braccia precisamente come nella Brachiella impudica. Quanto alla forma del corpo la specie somiglia alla Clavella fallax. (Tav. III, fig. 5). 
Un a1tro carattere distintivo offertomi dall' unico esemplare preso in esame è dato dal collo spesso ed allungato, piegato a curva in modo da abbracciare il corpo. Presenta le antenne del secondo paio divise all' estremità in due rami, ma come si vede dalla figura 6 a Tav. XX, uno dei rami è più lungo dell'altro e più sviluppato e formato alquanto diversamente che in altre specie. Le antenne del primo paio sono tri-articolate senza contare l'articolo basale ridotto a forma di piattello; terminano con tre setole. La bocca e le mascelle sono più o meno foggiate come nelle altre Clavelle. I piedi mascellari del secondo paio sono situati molto in alto rispetto a tutto il cefalotorace si da coprire col loro vistoso segmento basale gli organi boccali compreso il succhiatoio.

L'addome è di forma romboidale o piuttosto quadrata e presenta dal lato posteriore un tubercolo mediano poco saliente che rappresenta il post-addome.

I sacchi oviferi sono grossi e lunghi quanto il corpo.

All' estremità delle due alette poste alla base delle braccia sboccano le glandole allungate, sottili e comuni in quasi tutti i lerneopodidi e corrispondenti forse alle glandole del tegumento di altri crostacei.

Habitat. - Fu raccolta da me sulle arcate branchiali di Phicis blennioides B1. Schn.: estate igo3, Napoli.

Clavella Denticis? KRöYER, \&. Tav. XXI, fig. 6.

$$
\begin{gathered}
\text { Anchorella Denticis KRÖYER, I863 (2) p. } 296 \text { pl. XVI, fig. } 4 . \\
-\quad-\quad \text { HeLLER, I865 (1) p. } 243 . \\
-\quad-\quad \text { VOGT, I877(2) p. } 432 . \\
-\quad-\quad \text { BRIAN, } 1902 \text { (5) p. } 42 \text { con fig. }
\end{gathered}
$$

Descrizione della femmina.

Secondo Kröyer il capo è grande, bene distinto dal collo, e uguale a metà della sua lunghezza, non acuminato; collo abbastanza breve, gracile e sottile; braccia di mediocre lunghezza, col bottone più grosso del solito, ma più piatto, anteriormente poco convesso, posteriormente appena concavo; anello genitale più allungato, anteriormente stretto, posteriormente troncato; post-addome vistoso.

Gli esemplari da me esaminati non corrispondono in tutto alla descrizione che ci ha lasciato Kröyer. Vi sono differenze che è bene notare. Quell' autore distingue nella forma tipica un capo grande e un collo abbastanza breve. Invece nella mia forma il capo è piuttosto piccolo, acuminato al termine, il collo allungato, anzi tanto lungo da raggiungere e sopravanzare, in alcuni individui, la lunghezza dell'addome. Non credo tuttavia che queste differenze bastino per ritenere detta forma come nuova. Condivido l'opinione del compianto I. C. Thompson che esaminò, aderendo al mio invito, questi stessi esemplari, e che ebbe a determinarli per Anchorella Denticis.

Il capo è piccolo e in corrispondenza anche le parti boccali non sono molto sviluppate. Soltanto i piedi mascellari del secondo paio si lasciano bene distinguere e ne ho potuto studiare la struttura in uno dei miei esemplari. (Tav. XXI, fig. 6). Nè la base, nè l'uncino sono munite di spine o setole per quanto ho potuto vedere: ma il margine interno dell' uncino porta verso il mezzo un dente vistoso; questo margine è munito di piccoli peli e uncinetti nella maggior parte del suo 
percorso. La punta terminale dell' uncino è allungata e curva, porta il solito artiglio accessorio. Anche il margine interno del robusto articolo basale di questi piedi non è liscio sembra come seghettato o munito di dentini molto piccoli in tutto il suo percorso, ma questi sono evidenti solo con forti ingrandimenti. Non mi sono fatto un' idea abbastanza chiara delle altre parti boccali per poterle qui descrivere.

Secondo Kröyer il corpo della Clavella Denticis è lungo 2 linee ( $\mathrm{mm} .4 \cdot 36)$. Negli esemplari esaminati da me, le giovani femmine hanno una lunghezza variabile fra i 3 e $3 \frac{3}{4}$ e $4 \mathrm{~mm}$. In qualche individuo ho notato il collo più lungo dell' addome, ad esempio se il primo arriva a $3 \mathrm{~mm}$. il secondo è lungo appena $2 \mathrm{I} / 2 \mathrm{~mm}$.

Distributio et habitat. - Kröyer trovò per la prima volta questa specie sul Dentex argyrozona (cavità boccale) nell'Atlantico del sud. Di poi Heller riscontrò la sua presenza al Capo di Buona Speranza sul Dentex rupestris. Gli esemplari da me esaminati che riferisco a questa specie sono stati trovati aderenti alla pinna anale e dorsale del Chloropthalmus Agassizii Bp., pesce rarissimo nel nostro mare e pescato a Cornigliano Ligure, il 17 Dicembre I9oo.

Clavella Scombri KuRz, ㅇ․ Tav. X, fig. 6.

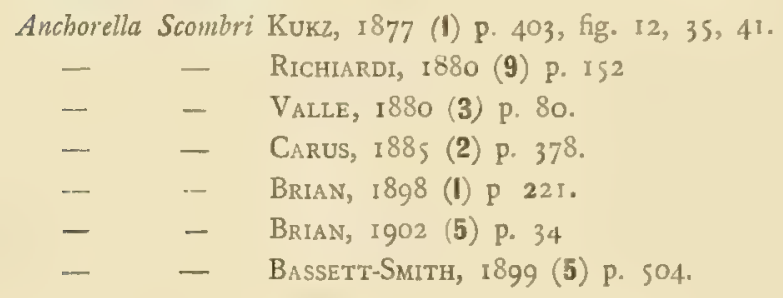

Distributio et habitat. - Kurz trovò un' unica femmina non completamente cresciuta sulle branchie di uno Scomber scombrus Linn. nell'Adriatico. Richiardi cita questa specie di copepode aderente alle lamine branchiali del detto pesce pel Mediterraneo. Valle non fa che ripetere la citazione di Kurz. Anche in Liguria fu rivenuta parecchie volte sull' ospite sopra riferito. Il D.r Damiani ne raccolse tre esemplari a Portoferraio sulle branchie di Scomber colias Linn.

Clavella macrotrachelus n. sp. $\subsetneq ~ \delta$. Tav. VIII, fig. 5. Tav. XXI, fig. I-4. Anchorella uncinata? BRTAN, 1898 (I) p. 22.

- Canthari Heller? Brian, Ig03 (9) p. 82.

- - $\quad$ Richiardi? Brtan, Ig03 (7) p. 9.

Descrizione della femmina.

È questa una piccola specie di Clavella che s'avvicina alquanto nel suo aspetto esterno alla forma di $\mathrm{Cl}$. centrodonti di Van Beneden, disegnata soltanto e non descritta da questo autore, specie non abbastanza conosciuta per potere quindi servire di base alla determinazione. Dato anche 1'ospite diverso su cui fu raccolta la nostra Clavella credo non errare ritenendola per forma nuova. La denomino $\mathrm{Cl}$. macrotrachehus per il carattere evidente del collo allungatissimo, seppure molto sottile. Anzi mi sembra un distintivo di detta specie l'essere il collo più lungo che il corpo: d'altro lato le braccia sono brevissime e riunite da 
un bottone chitinico. Questa forma l'ho creduta una Clavella uncinata Müller, tanto vi somiglia, se ne distacca però a causa delle sue dimensioni assai più piccole. Un individuo che ho raccolto a Napoli misurava le lunghezze seguenti: corpo $2 \mathrm{~mm}$.; sacchi oviferi $\mathrm{I} 1 / 2 \mathrm{~mm}$. Un altro individuo che ho trovato pure a Napoli aveva 1'addome lungo $21 / 2 \mathrm{~mm}$. e i sacchi oviferi $2 \mathrm{~mm}$.; il cefalotorace o collo $3 \mathrm{~mm}$.

Il maschio (Tav. XXI, fig. 2,4 ) è piccolissimo e vive attaccato (allo stato adulto) sul corpo della femmina.

Distributio et habitat. - Vive sulle branchie di Sargzus vulgaris Goeff., sovra cui ne raccolsi diversi esemplari l'estate I903, a Napoli. Un esemplare mi giunse pure da Portoferraio raccolto dal D.r Damiani sulle branchie di Sargus Rondeletii Cuv. e Vall., il 2 I Agosto Igo2. Questo individuo aveva il corpo lungo $x \mathrm{y} / 2 \mathrm{~mm}$. mentre il collo presentava una lunghezza quasi maggiore di $2 \mathrm{~mm}$.

A Genova ne raccolsi pure un esemplare il is Novembre rgor sulle branchie di Sargus Rondeletii Cuv. e Val. Esso ha l'addome lungo $2 \mathrm{~mm}$., il collo lungo $23 / 4 \mathrm{~mm}$.; i sacchi oviferi e il corpo tutto compreso $\mathrm{mm} .4^{\mathrm{Y}} / 4^{\text {. }}$

Somigliano a questa specie e ad essa riferisco anche altri esemplari che in precedenti lavori dubitavo potessero appartenere indifferentemente alla Clavella Canthari di Heller o alla $\mathrm{Cl}$. Canthari di Richiardi, perchè ritenevo queste due ultime specie simili. Questi esemplari sono i seguenti : un individuo femmina piccolissimo inviatomi da Portoferraio dal D. ${ }^{r}$ Damiani che lo raccolse sulle branchie di Cantharus lineatus Mont. (lungh. I ${ }^{1} / 2 \mathrm{~mm}$. senza sacchi oviferi) il 6 Novembre I902; e alcuni altri pure femmine, rinvenuti qui a Genova dal Prof. Parona parimente sul Cantharus lineatus Mont. il 3 Gennaio e il 27 Agosto rgo2. La lunghezza dell' addome arriva a mm. I $1 / 2$ circa. Il cefalotorace da solo misura $2 \mathrm{~mm}$. circa di lunghezza; esso è gracile, relativamente molto lungo. [Tutti e tre questi esemplari sono stati ritenuti per Cl. Canthari (Rich, e Hell.) in precedenti mie note]. Ho rinvenuto altresì un maschio fra di essi lungo mm. o,27. Colla $\mathrm{Cl}$. Canthari di Heller essi non possono assolutamente identificarsi, sia perchè sono molto più piccoli nelle dimensioni, sia perchè il collo invece di protendersi in avanti, è rivolto all' indietro sopra l'addome. Forse sono identici alla $\mathrm{Cl}$. Canthari di Richiardi, ma non è questo che un semplice dubbio non essendo stata quella specie mai descritta, nè avendo io potuto esaminare il materiale del Richiardi.

\section{Clavella Canthari RICHIARDI,}

$$
\begin{aligned}
& \text { Anchorella Canthari Richiard, I880 (9) P. I52. } \\
& \text { - } \quad \text { - VAlle, } 1880 \text { (3) p. } 78 . \\
& \text { - - Carus, I885 (2) p. } 378 . \\
& \text { - - Poche Franz, I9O2. }
\end{aligned}
$$

Specie non descritta.

Richiardi trovò tale Clavella aderente alle branchie di Cantharus lineatus Mont. e di C. orbicularis Cuv. e Val. (Mediterraneo). Valle la riscontrò alquanto frequente nell'Adriatico sulle branchie di Cantharus orbicularis Cuv. e Val.

Probabilmente la forma nuova, da me descritta col nome di $\mathrm{Cl}$. macrotrachelus n. sp., potrebbe essere identificata con questa specie, ma non mi fu 
possibile di esaminare il materiale raccolto da Richiardi per accertare se è vera questa mia supposizione. Per ciò neanche mi è possibile, per ora, verificare se la Clavella Canthari di Heller, 1865 (1) p. 242, p1. XXIV, fig. 6, debba essere ritenuta specie distinta o sinonima con questa.

b. Con due appendici addominali posteriori.

Clavella Triglae Claus, $q$ e $\sigma^{7}$.

Brachiella Triglae Ciaus, 1860 (3) Taf, I, fig. 6.

Anchorella Triglae KuRz, 1877 (1) p. 404, fig. I3-45, 22, 23, 46, 47.

-- $\quad$ - Richiardi, I880 (9) p. 152.

- - VALle, 1880 (3) p. 8r.

- - CARUS, I885 (2) p. 378

Brachiella Triglae BAssetT-Smith, i896, Journ. M. B. Assn. Plymouth, p. 163.

- - BASSETT-SMith, i 889 (5) p. 503.

- - Mrculicich, igos (2) p. 604.

Distributio et habitat. - Kurz trovò questa specie sulle branchie di Trigla lineata Linn. nel mare Adriatico. Richiardi la cita nel suo catalogo aderente alle arcate branchiali della Trigla obscura Linn. (Mediterraneo). Valle la dice specie alquanto rara sulle branchie della Trigla lineata Linn. e Trigla corax Bp. per l'Adriatico. Da Bassett-Smith è indicata per le coste inglesi (Plymouth) sulle branchie di una Trigla sp. ed è ritenuta da lui per una specie di Brachiella, come prima di lui ebbe già a giudicarla Claus.

Clavella paradoxa BENEDEN van, $Q$.

$$
\begin{aligned}
& \text { Anchorella paradoxa Beneden v., I8s I (3) pl. VI, fig. II7. } \\
& \text { - - BeNEDEN v., (I6) p. 37, tav. II, flg. } 6 . \\
& \text { - - Vogr, } 877 \text { (2) Arch. Zool. Exp. } 432 \\
& \text { - - BassetT-SMith, } 1896 \text { (1) pl. V, fig. 2, p. is. } \\
& \text { - } \quad \text { - Richiardi, I880 (9) p. IS2. } \\
& \text { - } \quad \text { - Carus, I885 (2) P. } 378 . \\
& \text { - - - BassetT-Smith, I899 (5) p. } 506 .
\end{aligned}
$$

Descrizione della femmina.

Si distingue questa specie dalle altre per la presenza di due appendici dal lato posteriore dell' addome. La testa, secondo Van Beneden, non è rigonfia e non esiste linea di confine che la separa dal cefalotorace. L'addome è globoso e nettamente separato dalla parte anteriore; è presso a poco tanto lungo quanto largo ed è terminato da due prolungamenti che hanno la metà di sua lunghezza; tra di loro si scorge anche un tubercolo post-addominale che non si vede bene che quando l'animale è giacente sul dorso. Tutto il corpo è straordinariamente molle sia nella porzione cefalica, sia nel rigonfiamento addominale. L'organo di presa costituito dalle due braccia è assai breve: appena si può dire che esiste una appendice. I sacchi oviferi hanno presso a poco il doppio di lunghezza dell' addome; contengono cinque o sei righe d'uova. La lunghezza del corpo di questa specie non ci è conosciuta. Il maschio è ignoto.

Distributio et habitat. - Van Beneden nota la Clavella paradoxa sulle branchie di Scomber scombrus Linn. per le coste del Belgio. Richiardi registra questa specie pel Mediterraneo, aderente alle lamine branchiali dello Scomber scombrus Linn. 
Bassett-Smith annovera tale parassita nella fauna di Plymouth, vivente sul detto ospite.

c. Con quattro appendici addominali posteriori.

Clavella hostilis HELLER, .

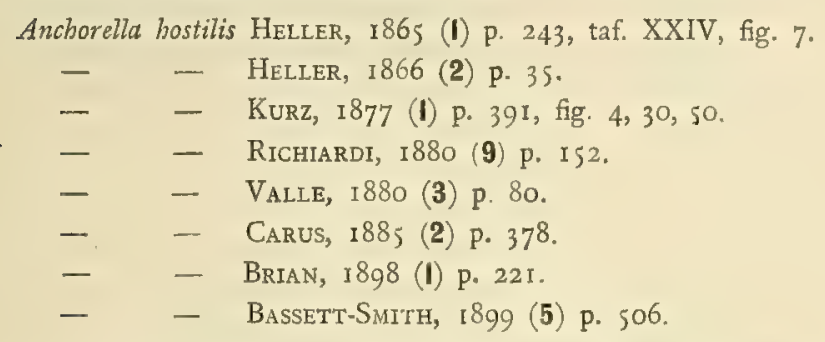

Distributio et habitat. - Da Heller fu descritta per la prima volta nel 1865 , come parassita dell' Umbrina cirrhosa e come speciale alla fauna del Mediterraneo. Per l'Adriatico sulle branchie dello stesso pesce è pure citata da Kurz. Richiardi 1a trovò aderente alla faccia interna delle arcate branchiali dell' Umbrina cirrhosa Linn e della Corvina nigra Cuv. Valle la dice assai comune, nell'Adriatico, sulle arcate branchiali della stessa Umbrina cirrhosa Linn. Io ebbi a citare questa specie anche per Genova essendo stata quivi raccolta sulle branchie del pesce ora nominato il 18 Ottobre 1889 e il 9 Luglio I889. A Napoli ne rinvenni un esemplare sempre su di quest' ospite indicato, nell' estate del I9o3.

Clavella Sciaence n. sp., @. Tav. X, fig. 2. Tav. XX, fig. I3, I4.

Brachiella neglecta RichiaRd, 1880 (9) p. 15 I (specie non descritta).

- - ?BRIAN, I 898 (I). p. 222.

? Brachiella sp. (Anchorella) BRIAN 1902 (5), p. 43.

? Anchorella sciaenophila HeLIer I86s (I) p. 243 pl. XXIV. fig. 8.

Descrizione della femmina.

In precedenti mie pubblicazioni avevo manifestato il dubbio che questa forma potesse riferirsi alla Brachiella neglecta del Richiardi sebbene non fosse stata da lui descritta, e mi appoggiavo per tale opinione, sull'identità dell'ospite: la Sciaena aquila Lac. Dopo più maturo esame, avendo ragione di ritenere il nostro lerneopodide piuttosto una Clavella che una Brachiella, io lo credo affine alla Clavella appendicolosa di Kröyer, ma lo descrivo tuttavia come una specie distinta e nuova non avendo dati sufficienti per identificarlo con quella. Questa nuova specie somiglia alquanto alla Clavella hostilis di Heller, come pure alla $\mathrm{Cl}$. sciaenophila dello stesso autore. Ciò non dimeno si distingue dalla prima, per la forma dell'addome distintamente foggiato a pera, e per avere le quattro appendici addominali presso a poco di uguale lunghezza: somiglierebbe, per questo carattere, piuttosto alla forma di Clavella sciaenophila, ma da essa tuttavia differisce per la presenza in più, di una quinta appendice mediana (post addome) frapposta ai sacchi oviferi.

Il corpo è a forma di pera; in avanti ristretto (Tav, X, fig. 2). Da una parte prende origine un collo abbastanza lungo e ispessito al termine, dall'altra sorgono due brevi braccia saldate tra loro e collegate al termine con un piccolo bottone chitinico, che 
serve per fissare l'animale alle branchie di Sciaena aquila Lac., e più precisamente sulle parti sporgenti delle arcate branchiali o sulle pareti della cavità branchiale. La parte posteriore del corpo è terminata da quattro appendici pressochè uguali e da una mediana più breve.

Nel capo (Tav. XX, fig. I4) si osservano due antenne posteriori potentemente chitiniche, assai sviluppate, che si assottigliano all' estremità e divise a questo punto nei soliti due rami. Sono curvate a formare quasi un angolo retto l' una di contro all' altra, e colle loro estremità anzichè toccarsi appena in punta, s'avanzano di un certo spazio l'una sopra l'altra. Le antenne anteriori sono sottili, cilindriche e presentansi formate di due o tre articoli. S'inseriscono sopra una formazione a piattello che fa le veci di articolo basale. L' estremità sembra munita di alcuni peli. Sono situate in dentro nel libero spazio interposto fra l'antenna posteriore e il rostro, e molto in avanti del cefalotorace. Un po' al disotto della loro inserzione e ai lati del rostro vedonsi le mascelle divise all' estremità in due appendici e un po' al di sotto munite di un terzo ramo, o meglio di due protuberanze brevi e terminate a punta (Tav. XX, fig. I3).

I piedi mascellari del primo paio sono robusti, 1' uno abbastanza vicino all'altro. Occupano i tre quarti circa della larghezza del capo. Essi ricoprono il rostro e le mascelle. Sono armate nel margine interno della base di una spina. Un' altra piccola spina setolosa si trova inserita sull' uncino.

Dimensioni: lunghezza del corpo. . . . . . . . $\mathrm{mm} .31 / 2$ lunghezza del collo. . . . . . . . $>31 / 2$

lunghezza delle appendici circa. . . . . . " $21 /$ larghezza massima del corpo . . . . . . 》 I $1 / 2$ lunghezza dell' appendice mediana . . . . » I.

I sacchi oviferi vistosi raggiungono sino a $4 \mathrm{~mm}$. di lunghezza.

Habitat. - Aderente alle branchie di Sciaena aquila Lac. Maggio 1891, Genova.

d. Con sei appendici addominali posteriori.

Clavella laciniata KRöy.? \&.

Anchorella laciniata KRöYER, 1863 (2) p. 305 , pl. XVI, fig. 7 .

- - Vogt, 1877 (2) Arch. Zool. Exp. p. 428.

- $\quad$ - ? BRIAN, I898 (I) p. 222.

- $\quad$ - Bassett-SMith, I899 (5) p. 506.

Questa specie va annoverata fra le Clavellae con post-addome bene marcato, e si distingue per sei appendici posteriori fissate all' addome.

Il capo, secondo Kröyer, è piccolo, poco distinto; il collo di lunghezza mediocre; le braccia del tutto ridotte, soltanto il bottone chitinico che risulta evidente è piatto, con brevissimo gambo; 1'anello genitale è gonfio senza forma ben distinta, con appendici posteriori grandi sub-coniche; post-addome piccolissimo. Il corpo supera la lunghezza di $4 \mathrm{~mm}$.

Secondo Kröyer questo copepode venne raccolto la prima volta sulle branchie di Acanthurus chirurgus nelle Indie occidentali. Io 1' ho citato per la Liguria 
da esemplari raccolti sulle branchie di Labrax lupus Cuv. e determinato da I. C. Thompson. Per quanto i caratteri esterni somiglino alquanto a quelli della specie di Kröyer sono tuttavia incerto sulla loro determinazione: a me sembrano somigliare alla Brachiella Merlucii B. S. ? tanto per la forma esterna quanto per i caratteri delle parti boccali.

c. Clavelle non descritte.

\section{Clavella Carusi Richiard,}

Anchorella Carusi, Richiard, I880 (9), p. Ist. - $\quad$-- Carus, I88s (2), p. 378.

Non descritta. Richiardi ha trovato questa specie aderente alla mucosa del1'apparecchio opercolare di Trigla lineata L. e $T$. corax Bp. Mediterraneo.

\section{Clavella clava RICHIARDI,}

Anchorella clava RICHIARDI, I880 (9), p. 152.

- $\quad$ - Carus, 1885 (2), p. 378.

Non descritta. Richiardi raccolse questa specie sulla Maena vulgaris Cuv. Val. e la trovò aderente al margine interno delle arcate branchiali. Mediterraneo.

\section{Clavella crassa RICHIARDI,}

Anchorella crassa Richiardi, 1880 (9), p. is2.

- Carus, i88s (2), p. 378.

Non descritta. Richiardi riscontrò questa Clavella aderente alla faccia interna delle arcate branchiali di Merlucius esculentus Risso. Mediterraneo.

\section{Clavella elongata RICHIARDI,}

Anchorella elonguta RICHIARDI, I880 (9), p. I52.

- - C Carus, i885 (2), p. 378 .

Non descritta. Richiardi trovò questa forma aderente alle lamine branchiali di Sargus annularis Linn. Mediterraneo.

\section{Clavella Lichiae RICHIARDI,}

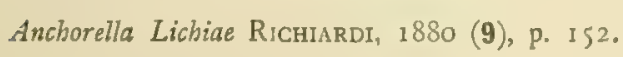

Non descritta. Questa nuova forma è ricordata dal Richiardi come aderente alle arcate branchiali della Lichia glauca Linn. Mediterraneo.

\section{Clavella subtilis RICHIARDI,}

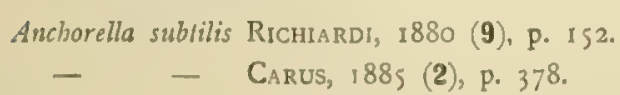

Non descritta. Questa Clavella vive, secondo Richiardi, aderente alle lamine branchiali dell' Umbrina cirrhosa Linn. Mediterraneo. 


\section{Clavella simplex RICHIARDI,}

Anchorella simplex Richiard, 1880 (9), p. I52.

$$
\text { - - Carus, } 1885 \text { (2), p. } 378 .
$$

Specie non descritta. Richiardi trovò questa Clavella aderente alle squame tra le pinne pettorali e le addominali del Merlucius esculentus Risso.

\section{Clavella tenuis RICHIARDI,}

Anchorella tenuis RICHIARDI, 1880 (9), p. 152.

$$
\text { - - Carus, i88s (2), p. } 378 \text {. }
$$

Specie non descritta. Richiardi rinvenne questa nuova forma aderente alle arcate branchiali del Pagellus erythrinus Cuv. L.a Clavella tenuis da me registrata per la Liguria 1898 (1) p. $22 \mathrm{I}$, è un copepode che ho inscritto qui nel genere Brachiella col nome nuovo di $B$. exigua n. sp.

\section{Gen. Naobranchia HessE,}

Naobranchia eygniformis HESSE, $q$ e $\sigma$. Tav. IX, fig. 3.

Naobranchia cygniformis Hesse. 1863 (3) IV, Ser., Tom. XX. Zool.. p. 122, pl. I, fig. I.

$$
\begin{aligned}
& \text { - } \quad \text { - Richiardi, I880 (9), p. iso. } \\
& \text { - - VAlLe, I880 (3), p. 71. } \\
& \text { - } \quad \text { C Carus, 1885, p. } 372 . \\
& \text { - } \quad \text { - BassetT-SMTth, I899 (5), p. } 507 . \\
& \text { - _ Brian Igo2 (5), p. } 34 . \\
& \text { - } \quad \text { - BRIAN, I903 (7), P. } 9 .
\end{aligned}
$$

Cestopoda amplectens KURZ, I877 (I) p. 407, fig. 16-2i, 34,49.

- - BassetT-SMith, 1899 (5) P. 507.

Distributio et habitat. - Questa specie fu trovata dall' Hesse sulle branchie del Pagellus erythrinus Cuv., lungo le coste al nord della Francia. Egli esaminò e descrisse soltanto le femmine. Kurz rinvenne questo copepode sulle branchie di Sargus annularis Linn. a Trieste. Da Valle è citato per l'Adriatico sullo stesso ospite. Richiardi registrando la specie pel Mediterraneo presenta diversi ospiti. Ne raccolse esemplari sulle branchie di Pagrus vulgaris Cuv. Val., di Sargus Rondeletii Cuv. Val., di S. anmularis Lin., e di Box boops Linn.

Io ricevetti esemplari presi sulle lamine branchiali di Box boops Linn., e di Smaris alcedo Cuv. e Val. dal D.r Damiani a Portoferraio; e ne esaminai altri tolti dalle branchie di un Sargus pescato presso Genova. Ne raccolsi pure un esemplare a Napoli sulle branchie di Sargus annularis Linn.

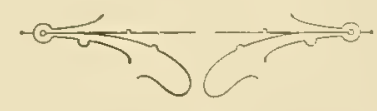




\title{
COROLOGIA
}

$\mathrm{O}$

\section{DISTRIBUZIONE DEI COPEPODI PARASSITI DEI PESCI}

\author{
NELLE ACQUE MARINE E DOLCI D'ITALIA
}

Lo studio da me fatto in non pochi anni e le indicazioni raccolte mi permettono di esporre un abbozzo della distribuzione geografica dei copepodi italiani, parassiti dei pesci.

Dal prospetto che riporto $\mathrm{mi}$ è dato riassumere come la fauna copepodologica italiana non sia scarsa in confronto di quelle di altri paesi. Per la condizione topografica dell' Italia nostra dobbiamo distinguere due regioni primarie marittime, quella del Mediterraneo propriamente detto e quella dell'Adriatico, porzione ben distinta del Mediterraneo, quanto dire la regione marittima ad Ovest e Sud dell' Italia e quella orientale (Adriatico) che dal lato ittiologico presenta specialità proprie.

Ad oltre 168 specie sarebbero quelle finora riscontrate nel Mediterraneo, delle quali $68 \mathrm{sp}$. sarebbero comuni al mare Adriatico. In questo sarebbero da indicare come proprie 9 sp. di copepodi, e con ciò non è a dire, che non si trovino anche sopra specie di pesci mediterranei, non essendovi pesci i quali vivano esclusivamente nel mare orientale italico.

Ho dovuto qui elencare un buon numero di specie di copepodi sotto 1' indicazione generale di Mediterraneo, perchè qualche naturalista straniero e il nostro Richiardi specialmente, nel citarli per la nostra fauna, non diedero notizia più precisa del luogo dove essi furono raccolti. Nel seguente quadro corologico sono notate alcune località che presentano un gran numero di copepodi parassiti, mentre altre ne contano pochissime. Non è a credere per questo, che le prime abbiano realmente una fauna più ricca ed abbondante delle altre. La differenza è dovuta unicamente all'essere alcuni punti delle nostre coste più popolati e più frequentati dagli studiosi come sarebbero Napoli, Genova, Trieste ecc., dove anche per la loro felice situazione geografica, riesce agevole intraprendere ricerche di fauna marina; mentre altre località, perchè più distanti da grandi centri popolati, sono state trascurate o quasi dimenticate e nessun studioso vi ha tentato ancora ricerche con proposito deliberato e continuo.

Riguardo ai copepodi di pesci esclusivi d'acqua dolce poco ho da dire: sono forme state indicate anche in altre regioni d'Europa e viventi su pesci comuni. Il loro numero per l'Italia ascende per ora soltanto a quattro specie. 


\section{N I ZZ A.}

Bomolochus Belones Burm.: Belone vulgaris; Hartmann (1870)

Caligus minimus Otro: Risso (1826).

? C. Lessonianus RIsso: Notidanus griseus; Risso (I826).

C. Rissoanus M. Edwardis (1840).

Lepeophtheirus Nordmanni M. Edwards: Orhagoriscus mola; M. Edwards (1840).

Dinematura producta $S_{\Gamma}$, e LüTK. : Lamna cornubica; HopE.

Cecrops Latreillii Leach: Orthagoriscus molu; Risso (1826)

? Pandarus Ronxii Risso: Carus (1885).

Anthosoma crassum ABILD. : Risso (1816), (1826).

Lernanthropus Kröyeri BEN : Labrax lupus; CLALS (1858).

Kröyeria lineata BEN。: Galeus canis; CLAUS (1858).

Nemesis Iamna Risso: Lamna cornubica; Risso (1826): L. comubica, Carcharias vulpes; Roux (1828).

Brachiella Thynni Cuv.: Hope.

\section{GENOVA e LIGURIA.}

Bomolochus cornutus Claus: Scomberesox Rondeletii; Brian (I8g8).

Caligodes laciniatus KRöY: Tylosurus imperialis; BRIAN (19O2)

Caligus curtus MülL. : Mugil cephalus.

C. minimus Otтo: Labrax lupus; Brian (1898); Clupea fina.

C. Lichiae mihi : Lichia amia.

C. ligusticus mihi : Box salpa.

C. productus DANA: Chrysophrys aurata; BRIAN (1898).

C. vexator Heller: Dentex vulgaris; Brian (I 898 ).

Lepeophtheirus Thompsoni BaIRD: Rhombus maximus; BRIAN (1898).

L. Nordmanni M. EDw.: Orthagoriscus mola.

Diphyllogaster Thompsoni BRIAN: Dicerobalis Giornae; BRIAN (1899).

Luttkenia Asterodermi Claus: Asterodermus elegans; BRIAN (1903) (7): Luvarus imperialis; Brian (1898).

Nesippus orientalis HeLI. : Mustelus Iaevis.

Elytrophora brachyptera Gerst.: Thymus vulgaris; BrIAN (1898).

Dinematura producta ST, et LüTK. : Selache maxima; BrIAN (1898).

D. Intifolia S.r. et LüTK.: Oxyrhina Spallanzanii; BrtaN ( 1898 ).

Echthrogalens coleoptratas ST. et LüTK. : Centrophorus granulosus; BRIAN (I S99) (1902).

Cecrops Latreillii Leach. Verany (1846): Orthagoriscus mola; Brian (1898).

Pandarus lugubris HELLER: Oxyrbina Spallanzanii; BrIAN (1902).

Perissopus dentatus ST. et LütK.: Galeus canis, Mustelus laevis, Carcharias lamia; Briax (I898).

Anthosoma erassum ABILD.: Oxyrlina Spallanzanii; BRIAN (Ig02).

Leruanthropus Mugilis BRIAN: Mugil auratus; BrIAN (1898).

L. Gisleri BEN. : Lichia amia, Umbrina cirrbosa, Sciaena aquila. BRIAN (1Sg8) (1902).

L. Kröyeri Ben.: Labrax lupus; BrJaN (I\&gS).

Kröyeria aenleata Gerst.? : Galeus canis; BRIAN (isg8).

Hatschekia Sargi Valle: Sargus sp., S. Salviani, S. Rondeletii ; Brian (Igo2).

H. Pagelli Bogueravei HrSSE?: Pagellus centrodontus; Goggio (1905).

H. Damianii mihi (Clavella Acanibulabri Exoleli Hesse?): Labrus; BrIaN (I 902 ).

Nemesis lamna Risso: Odontaspis ferox, Oxyrhina Spallanzanit, Alopias vulpes; Brian (IS98).

Cyenus pallidus BEN.: Conger vulgaris; BRIAN (I8g8)

Penienlus fistula Nordm.: Strgus Salviuni; BrIAN (1902).

Pennella filosa Cuv. (P. Costai Rich.): Xiphias gladius; Brıan (18g8). 
Lernaeenicus sp.: Netlastoma melanura.

Lernaeenicus sprattae Sow.: Clupea sprattus; BRIAN (1898).

Rebelula Edwardsii KöLl. : Macrourus calorbynchus; BRIAN (1903).

Peroderma cylindrieum Hell. : Chipea sp.; BrIAN (1898).

P. Bellottii Rich. : Scopelus caudispinosus; BRLAN (1902).

Medesicaste Triglarum KRöy. : Trigla lyra; BrIAN (1898)

Chondracanthus Merlucii Kröx.: Merlucius esculenlus; Brian (18g8).

Ch. angustatus HeLLeR: Uranoscopus scaber; BRIAN (I898).

Brachiella mallens RuD.: Torpedo narce.

B. exigna mihi : Pagellus erylbrinus

B. insidiosa Hell.? : Merlucius esculentus; Brtan (1898): Labrux lupus, Brama Rayi.

B. Thynni Cuv. Verany (1846): Thynnus thynnus: Brian (1898).

B. Merlueii Bass.-SM. : Merlucius esculenlus.

B. ramosa Rich.: Xiphias gladius; Brian (1902).

Clavella emarginata KRöY.: Clupea finta; Brian (1898).

Cl. Pagri Kröx.?: Pagrus vulgaris, Pagellus erylbrinus; Brian (1898).

Cl. strumosa mihi : Pagellus erytbrinus.

Cl. Sargi KuRz: Sargus annularis; Brian (1898).

Cl. fallax HeLL: : Denlex vulgaris; Brian (1898).

Cl. Denticis KRöy.?: Chlorophthalmus Agassizii; BRIAN (IgO2).

Cl. Scombri KuRz: Scomber scombrus; BRIAN (1898).

Cl. macrotrachelus mihi: Sargus Rondeletii, Cantharus lineatus.

C. hostilis HELI. : Umbrina cirrbosa; BRIAN (1898).

CI. Sciaenae mihi: Sciaena aquila

Cl. laciniata KRöY.?: Labrax lupus; BRIAN (1898).

Naobranchia eygniformis Hesse: Sargus; BrIAN (1903).

\title{
PORTOFERRAIO e ISOLA D'ELBA.
}

\author{
Psendoeneanthus Alosae Brian: Clupea alosa; Brian (1902). \\ Bomolochus Muraenae RıCH. : Muraena belena; BrIAN (I903). \\ B. unicirrus Rich. : Lichia glauca; BrIAN (1899) (1902). \\ B. Belones Burm.: Belone acus; Brian (1902). \\ B. cornatus Claus: Sayris Camperi, Cluper sardina, Exoccotus Rondeletii; BrIAN (1902) (1903). \\ C. minimus Orto: Labrax lupus; Brian (I899). \\ C. Lichiae mihi: Lichia amia. \\ C. ligusticus mihi: Box salpa, Sargus Rondeletii. \\ C. Pelamydis Kröy.: Pelamys sarda; BrIan ( 1899 ). \\ C. diaphanus Nordm.: 'rrigla corax, T. lineata, Pagellus mormyrus, Pagellus acarne. \\ C. vexator Heller: Dentex vulgaris, Pagrus vulgaris; Brian (1899). \\ C. Coryphaenae St. et LüTK. : Coryphaena bippurus: Brian (1899). \\ Lepeophtheirus Thompsoni BAIRD: Rhombus maximus, R. laevis; BRIAN (1899) \\ L. Nordmanni M. EDw. : Orthagoriscus mola \\ Liitkenia Asterodermi Claus (L. glabra): Luvarus imperialis; BRIAN (1903) (9). \\ Trehius candatus KRöY, : Raya macrorynchus: BRIAN (1902). \\ Elytrophora brachyptera GERST.: Thynnus thynnus; BRIAN (1899). \\ Dinematura prodncta ST, et Lürk. : Selache maxima. \\ D. Iatifolia ST. et LüTK : Oxyrbina Spallinzanii: BRTAN (I899). \\ Echthrogalens coleoptratus ST. et Lürk. : Charcharodon Rondeletii; BrIAN (1899). \\ Cecrops Latreillii Leach: Orthagoriscus mola; BrTan (1899). \\ Pandurus bicolor LEACH: Galeus canis; Brian (1899) \\ Anthosoma crassum ABILD.: Oxyrthina Spallanzanii; BRIAN (1899).
}


Lernanthropus Trachori BRIAN: Trachurus trachurus; BRIAN (1903).

L. Mugilis Brian: Mugil auralus; Brian (1903).

L. rolax Rich.: Charax puntazzo; Brian (1899).

L. scribae KRöY.: Serranus scriba.

L. Gisleri BEN.: Umbrina cirrhosa; BRIAN (1899).

Hatschekia Mulli BEN.: Mullus barbatus; BRIAN (1902).

H. Damianii mihi (Clavella Acanlholabri Exoleti Hesse y): Crenilabrus pavo; BrIAN (1903).

Nemesis lamna var. sinuata VALLE: Oxyrbina Spallanzanii.

Penuella crassicornis ST. et LüTK.: Prodelphinus euplurosyne; BRIAN (1903).

P. filosa Cuv. (P. costai RICH.) indiv. giovane: Xiphias gladius; BrIan (I899).

Lernaeolophus sultanus Nordu. : Maena vulgaris: BrIAN (1899).

Lernaea branchialis LINN.: Merlucius esculentus; BrIAN (1902).

Strabax monstrosus NoRDM.: Scorpaena scrofa; BRIAN (1899).

Chondracanthus cornutus MüLL. : Xiphias gladius.

Achtheres Grlei KR.: Galeus canis, Scylliun canicula, Sc. slellare; BRIAN (1899) (2) (3).

Brachiella malleus Rud.: Torpedo marmorala; Brias (1899) (3); Myliobalis nochula.

B. insidiosa Hell.?: Meriucius esculentus; BRIAN (1899).

B. Thynni Cuv.: Thynnus thymnus; Brian (1899)

B. impudiea Nordm, : Trigla corax, T. lineata; BRIAN (1903).

B. Morlucii BAss.-SMa: Merlucius esculentus.

B. elegans Rich. ?: Lichia amia; Brian (1899) (2) (3).

Clavella strumosa mihi: Pagellus erythrinus.

Cl. Characis Rich. : Charax punlazzo; Brian (1899) (2).

Cl. fallax Hell.: Dentex vulgaris; Brian (1902).

Cl. scombli KURz: Scomber colias; BRIAN (1902).

Cl. macrotrachelns mihi: Sargus Rondeletii, Cartharus lineatus.

Naobranchia eygniformis HESSE: Box boops; BRIAN (1902); Smaris alcedo.

\section{GOLFO DI NAPOLI.}

Bomolochus unicirius Rich.: Splyyraena vulgaris; Brian racc.

B. Belones Burmo: Belone acus; Brian racc.

Anchistrotos Gobii BrIan: Gobius capito; BrIAN racc.

Caligus Trachypteri Kr.: BrIan (IgO5).

C. diaphanus NoRdM:: Pagellus mormyrus, Trigla corax; BRIAN racc.

Lepeophtheirus Nordmanni M. EDw.: Orhagoriscus mola; BRIAN racc.

Nesippus orientalis Hell.: Alopias vulpes; Brian racc.

Nogagus Latreillii Leach.: Bran (Igo5).

Trebius candatus Kröy.: Acanthias vulgaris; Brian racc.

Elytrophora brachyptera GERST.: Thynnus thynnus; BRIAN racc.

Philorthagoriscus serratus KRöY.: Orthagoriscus mola; BRIAN racc.

Cecrops Latreillii LEACH. : COSTA, HOPE: Orthagoriscus mola; BRIAN racc.

Pandarus bicolor Leach. : Galeus canis; Brian racc.

Lernanthropus brevis RICH.: Sargus vulgaris; BRIAN racc.

L. Gisleri BEN. : Umbrina cirrhosa; BRIAN race.

Kröyeria linenta BEN.: Galeus canis; BRIAN racc.

Clavella Sargi VAlle: Sargus anmularis; BrLaN racc.

Hatschehia Riehiardii Goggro: Labrus fesiz'us; BRIAN racc.

Hatschekia sp: Crenilabrus pavo; Brian race.

Nemesis robusta Ben.: Spbyrua zygaena, Alopias vulpes; BrIan racc.

Eudactylina acuta BEN.: Squalina angelus, Acanthias vulgaris; Brian racc.

Plilichthys Xiphine Steenstr.: Xiphias gladius; Bergsoe (I865). 
Peniculus fistula Nordm.: Zeus faber; Nordmann (1832): Atherina, Boyeri; Brian racc.

Penuella rubra mihi: Orthagoriscus mola; Brian racc.

Rebelula Edwardsii Köle. : Macrourus colorbynchus; CoRnalia (1865).

Chondraeanthns Merlucii Kröy.: Hope; Merlucius vulgaris; Brian race

Brachiella exigua mihi: Pagellus erythrinus; Brian racc.

B. Merlucii BAss.-SM. : Merlucius esculentus; BRIAN racc.

Clavella uncinata Müll.: BRian (1905).

Clavella sp.?: Box salpa; BRIAN racc.

Cl. Sargi Kurz: Sargus vulgaris; Brian racc.

Cl. alata mihi: Phicis blennioides; BRIAN racc.

C1. macrotrachelus mihi: Sargus vulgaris; BRIAN racc.

Cl. fallax Hell. : Dentex vulgatis; Brian (IgO2).

Cl. hostilis HeLc. : Umbrina cirrbosa; Brian race.

Naobranchia eygniformis HESSE: Sargus anmularis; BRIAN racc.

\section{S I G I L I A.}

SENZA INDICAZTONE DETERMINATA DI LOCALITÀ.

Caligus Trachypteri KR. : Trachypterus sp.; KRöYER (1863).

Lepeophtheirus Grohmanni Kr.: Pleuronectes Grobmanni; KröYer (r863).

Elytrophora brachyptera GERST. (Arnaeus Thynni $\mathrm{K}_{\mathrm{R}}$ ): Thynnus thynnus; KRöYER (1863).

\section{PA LERMO.}

Lermanthropns Micropterygis Rich.: Micropleryx Dumerili; Richiardi (I885).

Hatschekia Cernae Goggro: Cerna gigas; GoggIo (I905).

\section{MESS I N A.}

Eneanthus Balistae Claus: Balistes sp.; Claus 1864.

Bomolochns cornutus Ciaus: Asterodermus coryphaenoides, Claus (1864).

Liitkenia Asterodermi Claus: Asterodermus coryphaenoides; Claus (1864).

Plilichthys Xiphiae Steenstr.: Xiphias gladius; Bergsoe (1865).

Rebelula Edwardsii KöLe.: Macrourus calorbynchus; KöLLIKER (I 853).

\section{SARDEGNA - GAGLIARI.}

Sphaerifer Corvinae Rich.: Corvina sp.; Leydig (I85I).

Nemesis lamna Risso: Oxyrbina Spallanzanii; Brian (1902). MazzA racc.

\section{GARLOFORTE.}

Dinematura latifolia $S_{\text {T. et }}$ LürT. : Alopias vulpes; PARONA racc.

ISOLA PIANA.

Pseulocyenus appendienlatus HeLL. : Thymnus thynnus; PAveSI race. 


\section{MEDITERRANEO.}

SENZA INDICAZIONE DETERMINATA DI LOCALITA.

Eneanthus Balistae Claus: Balisles capriscus; Rrchiardi (1880).

Bomolochus Mrraenae Rich.: Maraena helena; Richiardi (i 880 ).

B. unicirrus Rıch. : Lichia glauca, L. amia; Rrchiardi (1880).

B. Belones Burm. : Belone acus ; Richiardi (i880).

B. Soleae Claus: Solea rulgaris; Richiardi (I880)

B. cornutus Claus: Astrodermus elegans, Exoccetus volitans, Sayris Comperii, Clupea sardina; Richiardi (I880).

B. minimus Rıch. : Serranus scriba; Richiardi (i880).

B. oblongus Rıch.: Oblala melanura; Richiardi (1880).

Ergasilus nanus BEN.: Mugil cephalus, M. capito, M. auratus, M. saliens; Richiardi (1880).

Caligus minimus Otto: Labrax lupus; Richiardi (1880).

C. Alalongre Kröy.: Thynnus alalonga; CArus (I88;).

C. Pelamydis Kröy. : Pelamys sarda, Scomber scombrus; Richiardi (1880)

C. diaphanus Nord.: Pagellus mormyrus, P. erytbrinus, Trigla cuculus, T. corax, T. milvus, T. lineata, Platessa passer; RichIARdi (1880).

C. vexator Hell. : Dentex vulgaris, D. gibbosus, Pagrus vulgaris; Richiardi (1880).

C. Coryphanae ST. et LütK. : Coryphaena bippurus; Richtardi (r880).

C. Pharaonis Nordm.: Carus (188;).

C. affinis HeLler: Umbrina cirrhosa; RichlaRdi (I880)

C. Trachini Rich.: Trachinus draco; Richiardi (I880)

C. Trachuri Rich.: Trachurus trachurus; Richiardi (1880).

C. Serrani Rich.: Serranus gigas; Richiardi (I880).

C. Lepidopi Rıch. : Lepidopus caudatus; Richiard1 (I880).

C. Petersii Rich. : Carcharias lamia; Richiardi (I880).

Lepeophtheirus Thompsoni BAIRD: Rhonrbus laevis; $R$. maximus; RICHIARD! (I880).

L. Nordmanni M. Edw.: Orhagoriscus molae; HelleR (1865); Richiardi (1880).

Luitkenia Asterodermi Claus (L. glabra): Luvarus imperialis; Richiardi (IS80).

L. integra Rich.: Galeus canis, Mustelus equestris; Richiardi (I880).

Nogagus caelebs HeLL : HeLler (1865).

Demolens paradoxus Heli.: Helier (1865).

Elytrophora brachyptera Gerst: Thynus thynnus; Gerstaecker (1853); Heller (1865); Richiardi (I8So).

Dìnematura latifolia St, et Lütk.: Heller (IS6j): Prionodon glaucus; Richiardi (iS8o).

Ceerops Latreillii LEAcH.: Thynnus thynnus; Orlhagoriscus mola; RichIARd (i880).

Phyllophora crassa Rich.: Prionodon glaucus; Richiardi (i8so).

Pandarus bicolor LeAch.: Prionodon glaucus; Richiardi (I880).

P. lugubiis Heller (1865).

Perissopus dentatus ST. et LüTK. Mustelus equestris; Richtardi (1880).

Anthosoma crassum ABiLD.: Oxyrbina Spallanzanii; Richlardi (IS80).

Lernanthropus vorax Rich. : Charax punlazzo; RichIaRdi (i 879) (I880).

I. brevis Rich.: Sargus Rondeletii, S. annularis; Richiardi (1880).

I. scribae KRöY。: Serramus scriba; HeLLER (I \$6;).

L. Gisleri BEN. : Umbrina cirrhosa, Corvina nigra; Richiardi (I8So).

L. Kröyeri Ben. : Labrax lupus; RichIARdi (ISSo).

L. folinceus Rich.: Thyrsites pretiosus; RichIARDI (iS8O).

L. Tylosuri Rich.: Thylosurus imperialis; RichIARDI (I880).

Dichelestium sturionis Herm.: Acipenser sturio, A. Nacarii; Richlardi (IS80)

Kröyeria aculeata Gerst.: Prionodon glaucus; RichIARDI (1880).

Hatschekia Richiardii Goggro: Labrus merula; Goggro (1905).

H. Mulli BEN. : Mullus barbahus, M. surmuletus; Richiardi (I 879 ) (iS8o). 
H. obesa Rich. : Muraena belena; Richiardi (1880); Goggio (igos).

H. Cermae Goggio: Cerna aenea; Goggio (1905).

Hatschekia sp. Goggio: Labrus merula; Goggio (rgos).

Nemesis lamna Risso: Oxyrhina Spallanzanii, Lichia amia; Richtardi (I880): pesce-caile Heller (1865)

Cycnus? budegassue KröY.: Lophizus budzgassa; KröYer (1863).

Cyenus pallidus BEN: Conger vulgaris; RichiaRdi (1880).

Pseudoeyenis appendiculatus Heli. : Thynus pelamis; Richiardi (I880).

Plilichthys Xiphiae Steenstr. : Xiphias gladius; Richiardi (i880).

Polyrrhynelus Sciaenae Rich. Sciaena aquila; Richiarni (1876) (1.880).

P. Lichine Rich. : Lichia amia; Richiardi (1877) (1880).

P. Denticis Rich. : Dentex vulgaris; Richiardi (1877) (1880).

P. Pagri Rich. : Pagrus vilgaris: Richiandi (1877) (1880)

P. Pagelli Rich.: Pagellus mormyrus, P. erylbrinus; Richiardi (1877) (1880).

P. Baraldii Rich.; Chrysophrys aurata; Richiardi (1877) (1880).

P. Sieboldii Rich.: Box boops; Richiardi (1877) (1880).

P. minimus Rich.: Serranus hepatus; Richiardi (1877) (1880).

P. Grubeii Rich.: Sargus annularis; Richiardi (8877) (1880).

P. Agassizii Rich.: Charax punlazzo; RICHIs.RDI (1877) (I880).

P. Haeckelïi Rich.: Brama Rayi: Richiardi (I877) (I880).

P. Muraenae Rich. : Muraena belena; Richiardr (1877) (r880).

P. Edwardsii Rıch. : Serranus cabrilla; RichIaRdi (1876) (I 880).

P. Steenstrupii Rich. : Mullus barbatus, M. surmulelus; Richiardi (1880).

P. Doderleini Rich. : Labrus turdus; Richlakdi (1883).

P. Stromatei Rich.: Stromateus fialola; RichIARDI (i 880 ).

Sphaerifer Corvinae LEYD. : Corvina nigra, Sciaena aquila; RIChIARdi (1876) (I880).

S. Leydigii RICH. : Umbrina cirrhosa; RichlaRd (1877) (1880).

Peniculus fistula Nordm: Heller (1865).

Pennella crassicormis ST. et LütK.: HeLLER ( 1865 ).

P. fllosa Cuv. (P. costai Rich.): Xiphias gladius; Richinrdi (1880); Thymnus, Xiphias; Guérin (1840).

P. Remorre Murray: Carus (I885).

Tripaphylns Musteli Ben.: Mustelus equestris; Richlardi (I878) (1880)

Leimaeenicus vorax Rich.: Umbrina cirrbosa, Corvina nigra, Sciaena aquila; Richiardi (1877) (1880).

L. neglectus Rich : Mugil cephalus, M. capito, M. auratus, M. chelo, M. saliens; RiçHiardi (1877) (1880).

L. sprattae Sow.: Clupea sp.; HeLLER (I865)

L. Labrncis Rich.: Labrax lupus, Box boops, Trachurus trachurus; Richrardi (i 880).

L. Sargi Rich. : Sargus Salviani; Richiardi (1880).

Leruaea branchialis LiNs: : Merlucius esculentus; Rrchiardi (r88o).

L. Ninnii Rich.: Smaris vulgaris; Richiardi (I880)

Peroderma cylindricum HELL: Clupea sardina; Heller (I865); Richlard (1876) (1880).

P. Bellottii Rich : Scopelils Bernoiti; Richiardi (1881).

Medesieaste Triglarum KRöY.: Trigla lyra; Richtardi (1880).

Strabax monstrosus Nordm. : Scorpiena scrofa; Richiardi (1880).

Chondracanthus cornutus MüLL. : Solea vulgaris; Richiardi (1880).

Ch. Merlucii Kröy.: Merlucius esculentus; Richiardi (I880).

Ch. horridus Hell.: Gobius jozo; Heller (1865); Richiardi (1880).

Ch. Ninnil-Rich.: Gobius Panizzae; Richiards (I882).

Ch. angustatus HeLler: Uranoscopus scaber; HeLleR (I865).

Ch. Zei Delar : Zeus faber; Richiard (1880)

Ch. Lophii Johnst.: Lophius piscatorins; Richiardi (1880).

Achtheres Galei $\mathrm{K}_{R}$ : Scyllium slellare; Richlard (1880); Mustelus equestris (idem).

Brachiella insidiosa Hell.: Merlucius esculentus; Ruchiardi (1880).

B. Thynni Cuv.: Thynmus thynus: RrChlardi (1880).

B. neglecta Rich. : Sciaina aquila; RichiardI (I880). 
B. ramosa Rich.: Xiplras gladius; Richiardi (1880) (8); (9).

R. elegans Rich.: Liclsia glauca; RichisRDI (1880).

B. inconcinna RICH.: Raja maculala; RICHIARDI (I880).

B. minuta Rich.: Pagellus erilhrinus; Richiardi (1880),

B. obesa Rich.: Trigla corax; Richiardi (I880).

B. oblonga VALLE: Mugil cephalus, M. saliens; VALLE (I 880)

Clavella emarginata Kröy.; Alosa vulgaris; Richiard! (1880).

(1. Pagri Kröyं: P'agrus vulgaris; Kröyer (1863), P. vulgaris, Pagellus eryllurinus; Rıchiarir (1880).

Cl. Pagelli Kröx.?: Pagellus sp.; Kliôyer (1863); Pagellus mormyrus; Richiardı (1880).

C1. Sargi Kunz: Sargus Salviani, S. annularis; RIchIardi (1880)

Cl. Characis Rich.; Charax puntazzo; Richiardi (1880).

C1. fallax Hell: Denlex vulgaris; Helier (186;); Richiand (1880).

CI. Scombri Kurz: Scomber scombrus; Riciliardi (i880).

C. Canthari Rich : Cantharus linealus; C. orbicularis; RICHIARDI (1880)

Cl. Triglno Claus: : Trigla obscura: Richiadi ( 1880 ).

C1. paradoxa BEN.: Scomber scombrus; RichIARDI (1880)

C1. hostilis Hell. Umbrina cirrbosa; Heller (1865), Cl. cirhosa, Comina nigra; Richiardor (18So).

C1. Causi Rich.: Trigla lineala; T. corax; Richrardi (1880).

Cl. clava Rıch.: Maena vulgaris; Rıchiardi (1880).

C1. crassa Rich : Merlucius esculentus; RichIARDi (1880).

C1. elongate Rich.: Sargus annularis; Richiardi (I880).

C. Lichine Ricu. : Lichia glauca; Richiardr (1880).

Cl. subtilis Rich. : Umbrina cirrhosa; Richiardi (1880).

C1. simplex Rrcir.: Merlucius esculentus; RichuRdi (1S80).

Cl. tenuis Rıch.: Pagellus erylhrimus; Richiardi (i 880 ).

Nabranchia cygniformis Hesse: Pagrus vulgaris, Sargus Rondelelii, S. annularis, Box boops; Richiard (1880).

\section{ADRIATIGO - TRIESTE; ISTRIA E DALMAZIA.}

Gucanthus Marchesetti Valde: Motella tricirrula; Valde (I884).

Bomolochus Belones Burm.: Belone vilgaris; Hartmann (1870); Belone rosirala; Helier (I866); Valle (1880).

B. cormutus Claus. : Clupea papalina; Valle (1880).

Firgasilus manus Ben。: Mugil saliens; VALLE (1880).

Caligus minimus Otro; Labrax lupus; HeLler (1866); Valle (is\&o).

C. Pelamydis Kröy。: Pelamys sarda; Valle (1882).

C. diapluanus Nordom.: Trigla lineata; T. corax; T. aspera; T. lyra: Heller (IS66); VAlle (ISSo)

C. vexator Helc.: Dentex vulgaris; Heller (1865-1866); Valle (1880).

C. Coryphnenae St. et LüтK : Coryphaena pelagica; VAlle (1850).

C. aflinis Helder: Umhrina cirrbasa; Heller (1866); Valle (1880).

Tepeophtheirus pectoralis Nordm : Platessa-passer; Rhombus maximus; VALle (ISS4).

L. Nordmanui M. EDw.: Orthagoriscus molae: VAlle (I880).

L. Trygonis VAlIE; Tiygon pastinaca; VALIE ( 1882 ).

Liitkenia Asterodermi CLaus.: (Cecropsina glabra Hell.); HeLLfR (1865) (I866); Luvarus imperialis; VALL: (I880).

Trehius candatus Kröy.: Acanthias vulgaris; VAlle (I880).

Elytrophora brachyptera Gerst.: Thynnus thymus; HeLle? (1866); VALIE (1880).

Dinematura latifolia ST. et LütK: Carcbarodon Rondeletii; VALle (1880).

Cecrops Latreilli Leach : Thynnus thynnus; Helder (i 866); Valle (I8So).

Orthagoriscicola muricatus Kröy.: Orthagoriscus mola; VAlle (I880).

Perissopus dentatus Sr. et Lütk.: Mustelus pleblejus; Squalus Milberli; Hellek (1866); Valle (I88J). 
Anthosoma crassum ABIld.: Oxyrbina Spallanzanii; VAlle (1878) (1880)

Lernanthopins vorax Rich.: Charax puntazzo; VALle (1880).

L. brevis Rich.: Sargus Salviani; HeIder (1879); Sargus Salviani, S. Rondeletii, Oblata melanura; VALLE (I 880).

L. scribae KRöY.: Serranus scriba; HeLLfer (I866; VALLE (1880).

L. Gisleri Ben.: Umbrina cirrhosa, Corvina nigra; HeIder (1879); Valle (1880).

L. Krơyeri Ben.: Labrax lupus; Heller (1866); Hlider (1879); Valle (1880).

Dichelestium sturionis HERM.: Acipenser shrio; V $r$ LLE (1880).

Kröyeria lineata Ben.: Mustelus equestris; VALLE (1880).

Clavella Sargi Valle: Sargus Salviani; Valle (1882)

Hatschekia Mulli BeN。: Mullus barbalus, M. surmuletus; Valle (I880).

Nemesis lamna Risso: Carcharodon Rondeletii; Oxyrbina. Spallanzanii, Valle (I878), (I880).

N. Iamna var. simmata VALLE: Oxyrbina Spallañanii; VALLE (I878).

N. cobnsta BEN.: Trygon thalassia, Laeviraja oxyrbynchus, L. macrorbyncluus, Musiclus plebejus, M. equestris; VAlle (1880); Notidanus griseus; Valle (1884).

Cyenus pallidas BEN.: Conger vulgaris; VALLE (1884).

C. gracilis M. Edw., Helier (1866); Cerna gigas; Valle (1880).

Eudaetylina aenta BEN.: Squatina angelus; Acanthias vulgaris; VALLE (1880).

Plilichthys Xiphiae STEenstr.: Xiphias gladius; Valle (1880).

Polyrrhynchas Lichiae Rich.: Lichia ania; VALle (I880).

P. Agassizii Rich.: Cbarax puntazzo; Valle (1884).

P. Richiardii VAlle: Box salpa; Valle (1880)

P. Steenstimpii Rich: Mullus barbalus; VAlle (1880).

P. Stromatei Rich.: Stromateus fiatola; VALLE (1884).

Sphaerifer Corvinae Rich. Corvina nigra; VALLE (I880).

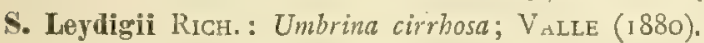

Peunella erassicornis Str. et Lürr.: Naucrates ductor, Xiphias gladius; VAlie (1880).

P. varians Stp. et Lürk. : (larva), Sepia officin, Loligo vulg., Eledone mosch.; WIERzEIJSKI (1877).

P. filosa Cuv. (P. Costai Rich.) Xiphias gladius; V.sLle (1882).

Tripaphylus musteli BEN.: Mustelus equestris; VALLE (1880)

Lernaeenicus gracilis Hell: Lichia amia; Heller (1865), VALLE (I880).

L. vorax RICH. : Umbrina cirrhosa; VALLE (:880).

L. negleetus Rich.; Mugil cephalus, M. saliens; VALLE (1880).

Lernaeolophus sultanus Nordm; Serranus scriba, S. cabrilla; Heller (1865), (1866); VAlte (1880).

Medesicaste Triglarum KRöx.: Trigla lineata; Heller ( I 866)

Strabax monstrosus NORDM.: Scorpaena scrofa; V $\triangle L L E$ (I882).

Chondracanthas cornntas Mürl.: Pleuronecles sp.; Heller (I 866).

Cl. Merlncii KRöy.: Merlucius; Heleer (1866); Merlucius esculenlus; VAlle "(880).

Ch. annulatis Olsson: Laeviraja oxyrbynchus; VAlle (1880).

Ch. Ninnii Rich.: Gobius Panizzae; Valle (1884).

Ch. angustatus Hell.: Uianoscopus scaber; Heller (1866); Schaub (1876); Valle (I8So).

Ch. Zei Delar.: Zells faber; Valle (I880),

Ch. Lophii Johnst.: Lophius piscatorius; Heller (1866), Valle (1880).

Charopinas Dalmannii Rerz.: Laeviraja macrorbyncluss, Dasybatis clavala; VALLE (I880).

Achtheres Galei KR. (Acht. Selachiorum Kurz): Mustelus laevis, Myliobatis aquila, Muslelus equestris; Kurz (1877), VALLE (1880),

Brachiella pastinacae Ben. : Myliobutis aquila; Kurz (1877); M. aquila; Rbinoplera marginala; VAlle (I 880).

B. insidiosa Hell.: Gadus sp. HeLier (1865); Merlucius esculentus; Valle (1882).

B. Thynni Cuv.: Thynnus thynnus; Heller (1866); Valde (I880); Mrculicich (1904).

B. impulica Nordm.: Trigla corax, Trigla lineala; Helder (1866); V' VLle (1880).

Clavella emarginata Kröy.: Alosa vulgaris; Kurz (1877); Valle (1880).

C. Incinata Müll: Merlucius sp.; Heller (1866).

C1. Pagelli Kröy.: Pagellus erylhrinus Cuv.; Heluer (1866); P. erythrinus, P. mormyrus; Valle (I8ủo). 
C1. Sargi Kurz.: Sargus annularis; KuRz. (1877); VALLE (1880).

c1. fallax Hell.: Denlex vulgaris; Heller (1806); Kurz (1877); Valle (I880).

CI. Scombri Kurz; Scomber scombrus; Kurz (1877); VAlle (1880).

Cl. Canthari Rich.: Cantharus orbicularis; Valle (1880).

CI. Triglae Cínus.: Trigla lineala; Kurz (1877); Trigla lineala, T. corax; VAt.Le (I880).

C. hostilis Helz.: Umbrina cirrbosa; Heleer (1866); Kurz (1877); VAlle (1880).

Nabranchia cygniformis HLSSE: Sargus annularis; KuRZ (1877); VALLE (1880).

\section{VENEZIA.}

Bomolochus Belones Burm.: Belone vulgaris; Har'rmann (I 870).

Chondracanthns Nimnii Rich.: Gobius Panizzae; VAlLe (1884).

\section{RI M I N I.}

Brachiella malleus RUd.: Torpedo marmorata; Nordmann (1832).

\section{F A NO.}

Ilchelestium sturionis H:RM. : Acipenser sturio.

\section{Prov. di VERONA (acque dolci).}

Ergasilns Sieboldii Nordm.: Cyprinus sp.; Garbini (1895).

Lamproglena opulchella Nordm.: Alburmus sp., Scardinius sp.; GARBixi (1S9j).

Lernaeocera cypriuacea Linn.: Esox, Cyprimus; Garbini (1895).

Actheres Percarum Nordm.: Perca sp.; Garbini. (1895).

\section{ITALIA (acque dolci)}

SENZA INDICAZIONE DETERMINATA DI LOCALITÀ.

Lamproglena pulchella Nordm. : Alburmus alliorella; Squalius car'edanus; Telestes Savignyi; Scurdinius erythrophibalmus; RichinRDi (1880).

Lermacocera cyprinacen Linn.: Esox lucius; Gobius fuvialilis; Rich ARdI (1877) (1880).

MASSA MARITTIMA (acque termali 26").

Lamproglena pulchella NoruM.: Telesles muticellus; ISSEL R. (IgOI).

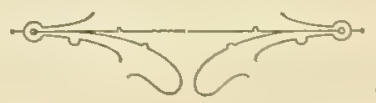




\section{BIBLIOGRAFIA}

I794. AbildgaARd P. C., 1. Beskrivelse af en Gjelleorm paa Brasens Krop. Lernaea anomala (Bramae). - Skrivt. Naturhist. Selsk. Kjöbenhavn III, 2, p. 57-58.

I794. - 2. Beskrivelse over tvende nye Monoculi Lin., Caligi Müll. (Caligus crassus et oblongus). - Skrivt. Naturhist. Selsk. Kjöbenhavn. III, 2, p. $46-54$.

1865. Agassiz A., Sur un Pennella, parasite de l'Orthagoriscus mola. - Illustrated Catalogue of the Museum of Comparat Zoology.... Cambridge, p. 87.

L'a. ha trovato vivente in associazione colla Pennella una Campanularia (Eucope parasitica).

1855. AldER and HANCOCK, A monograph of the british nudibranchiate mollusca. Ray Society. Part. VII, I855, p. 26-27, pl. 45, fig. 8-IO.

Gli autori descrivono brevemente due specie diverse di copepodi parassiti infestanti le Dorilidae: ossia una specie di Bomolochus raccolta nella Doris pilosa (cavità addominale), e una specie di Ergasilus parassita della Doris tuberculata. Essi ritengono queste due forme nuove: non le nominano, ma le illustrano a tav. 45. La prima specie è rappresentata dalle fig. 8 , 9 e la seconda dalla fig. ro.

In ultimo fanno cenno di un'altra specie di Ergasilus parassita dell' Antiopa cristata da loro trovata nel mezzodi delt' Inghilterra di cui notano semplicemente che « had decp orange-coloured ovigerous lobes".

Gli autori a proposito della Tethys aggiungono che è șolita ad essere infestata da un grande entomostrace e ricordano come un individuo affetto in tal modo dal suo parassita è rappresentato in figura dal Delle Chiaje nelle sue Memoires (copied in Mrs Gray's Figures of Molluscous Animals: pl. 210, fig. I).

1847. Allmann G., 1. Description of a new genus and Species of Entomostraca, Notodelphys. - Annals and Magazine of Naturalhistory. Vol. XX. N. ${ }^{\circ}$ I 30 . Juli 1847.

E questo un livoro molio interessante ma confuso.

1847. - 2. On the development of Notodelphys Allm., a new genus of Entomostraca. - Report Brit. Associat. f. Advanc. of Science, I 7. Meeting. Transact. p. 74 .

1905. Anthony R., et Calvet L., 1. Note sur les Pennella Balaenopterae (Kor. et Daniels.) recueilliẹs sur le Balaenoptera physalus (Linn.) de Cette (Octobre 1904). - Bull. Mus. Hist. Nat. Paris, p. I98-200.

1905. - - 2. Recherches faites sur le Cétacé capturé a Cette le 6 Octobre 1904 (Balaenoptera physalus Linné) ecc.... Parasites extérieurs (Pennella Balaenopterae K. et D.) - Bull. Soc. Philom. Paris (9) T. 7, p. $75^{-8}, 3$ figg. 
1826. Audouin J. V. et M. Edwards H., Mémoire sur 1a Nicothoé, animal singulier qui suce le sang des homards. - Ann. d. Scienc. Natur., I sér., Vol. IX, p. 345-359, avec. pl. fig. I-9.

I883. Aurivilitus C.W.S., Bidrag till Kännedomen om Krustaceer, som lefva hos Mollusker och Tunikater. - Akademisk Athandling, Stockholm.

I847. BaIRd W., 1. Lepeoptheirus Stromii Baird osp. Salmo. - Trans. Berwick. Nat. Club.

? - 2. Pennella pustulosa? Baird. - Ann, Nat. Hist. XIX, p. 280.

L'a. ha descritto con questo nome una specie di Pemnella che Steenstrup e Lütken (i86I) ritengono potersi avvicinare alla $P$. varians Stp. e Ltk.

1850. - 3. The Natural History of the British Entomostraca. - London.

$\dot{E}$ un lavoro d'indole generale, con una completa rassegna specigrafica delie specie di copepodi parassiti viventi in Inghilterra e note fino al i8jo. Vi ì studiata la morfologia esterna di ogni singola specie. Presenta un certo interesse anche la parte storica che riguarda in modo particolare la famiglia dei caligidi e quella dei lerneidi e che riguarda anche i generi e le specie. Sono descritte alcune forme nuove. Caligus cenlrodonti Baird; Lepeophtheirus Strombii Baird; L. obscurus Baird; Chalimus Scombri Baird. Quest' ultimo non è altro che la larva del Caligus curtus Müll.

1861. - 4. Note on the Lernaea cyclopterina occurring in the gills of the Cyclopterus spinosus, a fish from Greenland. - Proceed. of the Zoolog. Soc. of. London, p. 239; Annals of Nat. Hist., 3 ser., VIII, p. 496.

I744. BAKER H., A letter concerning a new discovered Sea-Insect, which he calls the Eye-sucker. - Philosoph. Transact. of the Royal Soc. of London, Vol. 43, N.r 472, p. 35-36, c. tab.

L'a. tratta dei cosidetti Eyesuker corrispondenti a forme della specie Lernaeonema spratta (Sowerby), che egli dice trovate " fixed by the snout to the eyes of a sprat $\mathrm{m}$.

1765. BAster, Opuscula subseciva II, p. I38.

Intorno alla Lernaea Basteri.

1896. Bassett-Smith P. W., 1. Notes on the Parasitic Copepoda of Fish obtained at Plymouth with Descriptions of new Species. - Ann. of Nat. Hist. (6) Vol. I8. July, p. 8-i6. With 4 pls. [Abstr. Journ. R. Micr. Soc. London. 1896. P. 5, p. 523] [II (7 nn.) spp.].

Le 7 nuove specie che l'a. descrive sono le seguenti: Caligus scomberi n. sp.; C. brevipedis n. sp.; Lepeophtheirus pollachious n. sp.; Lernea lusci n. sp.; Chondracanthus clavatus n. sp.; Brachiella merlucci n sp; Anch. quadrata n. sp.

1898. - 2. Some new Parasitic Copepods found on Fish at Bombay. - Ann. of Nat. Hist. (7), Vol. I, Jan. p. I-I 5-I7. With 7 pls. (I4 nn. spp.; n. g. Helleria).

Le 14 nuove specie portano questi nomi: Bonolochus triceros n. sp.; $B$ tetradonis n. sp.; Caligus parvus n. sp.; C. cybii n. sp.; C. birsutus n. sp ; C. Phipsoni n. sp.; C. lonğicaudus n. sp.; g. n. Cybicola (Helleria); Cyb. armata n. sp.; Lernanthropus trifoliatus n. sp ; Lernaeonema poly:emi n sp.; Peroderma branchiata n. sp.; Chondracanthus elongatus: Brachiella appendiculosa n. sp. 
1898. Bassett-Smith P. W., 3. Further new Parasitic Copepods found on Fish in the Indo-tropical Region. - Ann. of Nat. Hist. (7), Vol. 2, Aug., p. 77-97-98. With 4 pls. (I I nn. spp.; n. g. Pseudoclavella).

I898, - 4. Some new or rare Parasitic Copepods found on Fihs in the IndoTropic Region. - Ann. of Nat. Hist. (7), Vol. 2 Nov., p. 357-371-372. With 3 pls. [8 (4 nni.) spp.].

Le specie nuove descritte dall'autore sono: Caligus longipedis n. sp; C. rolushlus n. sp.; Caligodes carangis n. sp.; Lernanthropus nudus n. sp.

I899. - 5. A Systematic Description of Parasitic Copepoda found on Fishes, with an Enumeration of the Known Species. - Proc. Zool. Soc. London, 189.9. P. I I, p. 438-507. With I pl.

Dovrebbe formare un elenco generale di tutte le forme conosciute dei cop. paras. dti pesci, ma molte specie sono state dimenticate dall" a. Si yeda la critica che ne ha fatto Poche Franz nel Zool. Anz, Vol. 26, N. 685, p. 8-20, il quale ebbe a rilcvarvi non poche sviste e dimenticanze. (E un lavoro, ció non dimeno, che, emendato, tornerebbe utilissimo a chi s'occupa di sistematica e di faunistica dei copepodi parassiti).

Molte sono le specie e i gencri nuovi che l'autore qui cita e che ha descritto in precedenti sue memorie.

1903. - 6. On new Par. Cop. from Zanzibar and East Africa, collected by M.r Cyril Crossland. Proc. Zool. Soc.-London, I903, Vol. I, p. I04-109, 2 fig.

(2. nn. spp. in: Chondrocarpus; Ventriculina nn. gg.).

1904. Baudouin M., 1. Le Lernaeenicus sprattae, parasite de la Sardine en Vendée. - C. R. Acad. Sc. Paris, T. I39, p. 998-1000.

(L. sardinae n. sp. avec restriction).

1905. - 2. Les parasites de la Sardine, dans Rev. Scient., Paris, n. 23, ro Juin, p. 7I5-722, 4 fgs.

1905. - 3. Nouvelles observations sur le Lernaeenicus. Copépode parasite de la Sardine. - Bull. Mus. Hist. Nat. Paris, p. 299-300.

(L. encrassicoli).

1850. Beneden P. J. van, 1. Mémoire sur le développement et l'organisation des Nicothoés.-- Nouv. Mémoires de l'Acad. de Bruxelles, T. XXIV, avec pl.; oppure Ann. d. Scienc. Natur., 3. sér., Zoologie, Vol. XIII, p. 354-377 avec pl.

1851. - 2. Note sur un Crustacé parasite nouveau, avec l'enumeration des espèces de cette classe qu'on observe sur les poissons du littoral de Belgique. - Bull. de 1'Acad. de Belgique, T. I8, n. I, p. 286-29o avec pl.; oppure l'Institut, XIX, p. 285-286.

L'a. descrive il Lernaeonema musteli par. del Mustelus vulgaris, e assegna alla femmina if mm. di lungh. senza le appendici addominali (queșt' ultime' misurano Is mm); inoltre descrive anche il maschio. 
185I. BENEDEN P. J. van, 3. Recherches sur quelques crustacés inférieurs. - Ann. d. Scienc. Natur., 3 sér., Zoologie, Vol. XVI, p. 7I-I3I, avec 5 pl.

L' a. tratta con qualche diffusione delle specie seguenti; Nicothoe Ashuci Miln. Edw.; Caligus Hippoglossi; C. elegans van Ben.; C. gracilis van Ben.; Pandarus bicolor; Dichelestium Sturionis; Ergasilina robusin van Ben.; Clavella Mulli van Ben.; C. Hippoglossi Kr.; Lernanthropus Kroyeri van Ben.; Chondracanthus gibbosus Kr.; Ch. cornutus Müll.; Ch. zei; Ch. Triglae; Ch. Soleae; Brachiella Pastinacae van Ben.; Lernaeopoda Galei Kr.; Anchor ella emarginata $\mathrm{Kr}$.; A. paradoza van Ben.; A. uncinala Müll.; A. rugosa Kr.; Lernaconema musteli; Lernaea branchialis.

1852. - 4. Note sur quelques parasites d'un poisson rare sur nos côtes (le maigre d'Europe) Sciaena aquila Cuv. - Acad. Roy. de Belg., Extr., T. XIX, n。 9 des Bulletins.

L'a, descrive la no sp. Lernanthropus Gisteri, maschio e femmina, illustrandola con figura.

1852. - 5. Note sur un nouveau genre de Crustacé parasite de la famille des Peltocéphales. - Acad. Roy. de Belg., Extr., T. XIX, n. I i des Bulletins, I sér., III partie, p. $462-467$.

L'a. descrive il Sciaenophilus tenuis van Ben, che vive nella cavità branchiale di Sciaena aquila. Il suo corpo ha il $4 .^{\circ}$ paio di piedi liberio Lungh. tot. $14 \mathrm{~mm}$. colle app. caud.; $6 \mathrm{~mm}$. testa, torace, addome; $8 \mathrm{inm}$. coda.

1853. - 6. Note sur un nouveau genre de Crustacé parasite, Eudactylina. (E. acuta). - Bull. Acad. Roy. Belg., T. XX, pt. I, p. 235-239.

1853. - \% Notice sur un genre nouveau de la tribu des Caligiens, genre Kröyeria van Bened. - Acad. Roy. de Belg., Extr. des Bulletins, T. XX, n. I, p. 23-3o, avec pl.

L'a. descrive la n. sp. Kroyeria lineata.

1853. - 8. Note sur un nouveau genre de Crustacé parasite, Pagodina. Acad. Roy. de Belg.. lixtr. du T. XX, n. 4 des Bulletins.

L'a. descrive la Pagodina robusta n. sp. che entra nella sinonimia della Nemesis robusta.

1854. - ?. Notice sur un nouveau genre de Siphonostome, genre Congericola. - Bull. de 1'Acad. de Belg., T. XXI, n. 2, p. 583-589.

L'a. descrive la sp. Congericola pallida v. B., il cui nome generico più tardi è stato cambiato in Cycnus pallidus; abita le branchie del Congro. Ha $8 \mathrm{~mm}$. di lungh. compresi i tubi oviferi. Il $\mathrm{O}^{x} \mathrm{e}^{\mathrm{x}} / 4$ o $\mathrm{t} / \mathrm{s}$ della lungh. del corpo della femmina.

1855. - 10. Sur le Cecrops Latreillei. - Bull. de 1'Acad. de Belg., T. XXII, n. 2 , p. $523-527$.

1857. - 11. Notice sur un Lernanthrope nouveau du Serranus Goliath. - Acad. Roy. de Belg. Extr. du T. XXIV, n. I des Bulletins, av. pl.

L'a. descrive il Lernanthropus Petersi v. Ben. $q$ e ' $0^{x}$, vivente sopra il Serranus goliath e raccolta a Mozambicco. 
1857. BENEDEN P. J. van, 12. Notice sur un nouveau Dinémoure provenant du Scimnus glacialis. Extr. des Bulletins de 1'Acad. Roy. de Belg., T. XXIV, n. 2, ser. I, p. 226-235, avec pl.

L'a. descrive la Dinemoura elongata v. B., e ne dä il diseguo.

1860. - 18. Notice sur un nouveau genre de Crustacé Lernéen. - Bull. de l’Acad. de Belg., 2 sér., T. IX, p. I5I-I63, avec pl.

L'a. descrive l'Enterocola fulgens v. B. che vive entro il primo compartimento d' un tunicato composto, ossia nell'Apitidium ficus e $A$. ficoüles van Beneden.

186r. - 14. Recherches sur la faune littorale de Belgique: Crustacés. - Mém. de l'Acad. Roy. de Belg., T. XXXIII, I861.

I870. - 10. Les Cétacés, leurs commensaux et leurs parasites. Bull. Acad. de Belg., 2 sér., T. XXIX, p. 347-368.

Fra i cop. parass, di cetacei l'a. cita:

1. Permella balaenoptera Sars (Iles Loffoden, Islanda) Balaenoptera musculus

2. Pennella crassiconis Stp. et Ltk. sopra dell'Hyperoodon (rostratum) $=$ Butykopf.

3. Lernaeonema nodicornis Stp. et Ltk. sopra Delphinus delphis. •

4. Pennella pustulosa Baird. sopra Delphinus.....

I870. - 16. Les poissons des côtes de Belgique. Leurs parasites et leurs commensaux. Bruxelles.

Troviamo in questo pregiato lavoro citati moltissimi cop parassiti coll'indicazione precisa del loro ospite e delle parti del pesce su cui vivono.

I875. - 17. Les commensaux et les parasites dans le régne animal. Paris.

1883. - 18. Animal Parassites and Messmates. International Scientific Series, XIX, D. Appleton \& Co.

189I. - 19. Deux nouveaux Lernéopodiens recueillis I'un aux Açores, l'autre sur les côtes du Sénégal: Brachiella Chavesii, Br. Chevreuxii. Avec deux p1. - Bull. Acad. Roy. de Belg., 3 sér., T. XXII, n. 7, p. 23-35; oppure Two new Lernaeopoda (Brachiella). - Abstr. in: Journ. R. Microsc. Soc. London, I891: P. 6, p. 738.

L'a. descrive Brachiella Chavesii n. sp. $q$ d , specie che, secondo me, sarebbe vicina alla Brachiella Thynni. Per errore Beneden ha indicato cone ospite di questa specie un Ceralopterus (Vedi correzione "Quelques n. caligides de la côte d'Afrique » p 249. Nota. 1902). Questa Brachiella proviene dalla Baia di Dakar.

Inoltre descrive la Brachiella Cherreuxii n. sp.

1892. - 20. Quelques nouveaux Caligides de la côte d'Afrique et de l'Archípel des Açores. Avec 4 pl. et I incis. - Bull. Acad. Roy. de Belg., 3 sér., T. XXIII, n. 9-10, p. $241-262$.

L'a. descrive 5 n. sp.: Caligus Dakari; Nogagus angustatus; Calina brachyura; Pupulina fores; Caligera difficilis. 
1892. BENEDEN P. J. van, 21. Le mâle de certains Caligidés et un nouveau genre de cette famille (Chlamys n. g. incisus n. sp.) Avec 2 pl. - Bull. Acad. Roy de Belg., 3 sér., T. XXIII, n. 3, p. 220-235.

L'a. fa conoscere in questa nota: I. il maschio di Pandarus cranchii; 2. il maschio e la fenmina di Pandarus affinis sp. n.; 3. il gen. n. Chlanys incisus (affine al Perissopus); 4. il maschio della Dinemoura elongafa. E da notarsi quanto segue: nell' introduzione Beneden scrive che Burmeister (1835) avendo ricevuto dal D." Stannius alcuni parassiti raccolti ad Helgoland, su uno squalo, tra questi distinse due forme. Riferi l'una al gen. Pandarus, l'altra al gen. Dinemoure. Queste due forme sono il maschio e la femmina d'una medesima specie. Dana (U. S. Expl. Exped. Crustacés) ha commesso un errore consimile: lo Spicilligus curticaudis di Dana è il of d'uno dei tre Pandarus che cgli descrive. G. M. Thomson (1890) ha fatto la medesima osservazione in Nuova Zclanda, sul Nogagus elongulus di Heller, che è il maschio di Panlarus dentalus e sospetta anzi che i diversi Nogagus sono maschi; ma questo naturalista forse va troppo oltre; poiché l'a. ha veduto Nogagus accoppiati, trovati nel materiale ricevuto daile Acores, che non presentano che le differenze sessuali ordinarie in altri caligidi.

1869. Beneden P. J. van et LAcordatre M. Th., Développ. des gen. Anchorella, Lernaeopoda, Brachiella et Hessia, con I tav: - Bull. Acad. Roy. de Belg., 2 sér., T. XXVIII, p. 223-254.

I870. BENEDEN E. van., 1. Recherches sur l'embryog. des Crustacés:

III a) Développ. de l'œuf et de l'embr. des Sacculines (Sacculina carcini Thomps.) - Bull. Acad. Roy. de Belg., 2 sér., I. XXIX,

IV b) Développ. des genres Anchorella, Lernaeopoda, Brachiella et Hessia. p. 223. - Bull. Acad. Roy. de Belg., 2 sér. T. XXIX.

I880. - 2. De l'existence d'un appareil vasculaire à sang rouge dans quelques Crustacés. - Zoolog. Anzeigers. N. 48, III Iahrg.

L'a. fa una distinzione fra liquido plasmatico (sistema plasmatico) o liquido cavitario che ha l'ufficio del plasma extravasato dei vertcbrati e il liquido ematico (apparecchio ematico). Secondo l'aut. non esisteva in nessun altro crostaceo o artrop ido, nemmeno copepode libero sistema vascolare omologo a quello dei Lermanthropus, Clavella e Congericola; recentemente fu riscontrato dallo Steuer anche per altro genere $\mathbf{M y}^{\mathrm{y}}$ tilycola. - Si potrebbe chiamare "liquide hematique " questo sangue che non è tale, come nei vertebrati, nei vermi e nei crostacei ma servirebbe a trasmettere al liquido cavitario l'ossigeno àssorbito, e a facilitare l' eliminazione dell' acido carbonico; avrebbe l'ufficio dei globuli rossi nei vertebr. „ p. 58.

I862. BenEden E. et Bessels É., Mémoire sur la formation du blastoderme chez les Amphipodes, les Lernéens et les Copépodes. - Mém. (cour.) de l'Acad. Roy. de Belg., T. XXXIV.

1866. BERGH, Copepodi parassiti vicini al gen. Splanchnotrophus. - Malakol. Unters. Heft. Io, p. 408.

Phidiana lynceus og Imaila monstrosa. - Naturh. Foren. Vidensk. Meddel. I866, p. I I6. 
I 864. BERgsoe V., Philichthys Xiphiae Stp., Monographisk Fremstillet. - Naturhist. Tidsskrift 3 Raek., 3 Bind, p. 87-I30, tab. I3, Kjöbenhavn.; (oppure Ann. des Scienc. Natur., 5 sêr. Zoologie, Vol. III, pag. 2 I3, pl. I, I 865 ).

E un' estesa e splendida monografia di questa specie scritta in danese. Vi si trova però unito un sunto in latino Repetilio brevis colla descrizione del maschio e della femmina.

i8i6. Blainville H. D., 1. Artikel Lernaea in: Dictionn. d. Scienc. Natur., Vol. XXVI, p. I12, ff., I816 (e 1823).

1822. - 2. Mémoires sur les Lernées. - Journal de Physique, T. 95, p: 372-380, et p. 437-447, avec I pl.

1674. Boccone, Ricerche e osservazioni naturali. Amsterdam, i674; oppure Observ. nat. Paris, 167I. (Intorno ad un Hirudo sive Acus cauda utrinque pennata).

1860. BEECK A., Artotrogus og Asterocheres. - Förhandlingar of Videnskabs Selskabet in Christiania, aar 1859; p. 17I-I81, pl. I, 2.

L'a, descrive Artotrogus orbicularis su di una Doris; Asterocheres Lilljeborgi su di un Echinaster sanguinolentus.

I898. Bonnier J., Sur un type nouveau de Copépode gallicole Pionodesmodes n. g., phormosomae n. sp. - C. R. Acad. Sc., Paris, T. I26, n. 10, p. 769-77I. Extr. Revue Scientif. (4), T. 9, n. I 2, p. 370-37I.

1830. Bosc, Hist. nat. des Crustacés, t. II.

1878-80. BRAdy G. G., 1. Monograph of the free a. semi-parasitic Copepoda of the British Islands, 3 vols. London Ray Society, I878-80 (Vol. x, I878; Vol. 2, I880; Vol. 3, I880).

1883. - 2. Report on the Copepoda obtained by H. M. S. Challenger Expedition etc. in Reports Scientif. Resultats. Voyage Challenger, Zool. Vol. 8, n. XXIII, With 55 pl. (I 42 p.)

[New Zealand Copepoda of the Challenger Expedition. Extr. from. G. G. Brady's Monograph in: The New Zeal. Journ. of Sc. Vol. I, n. I, p. $35^{-} 39 ;$ n. 3, p. II 7-1I9].

L'a. descrive le seguenti specie: Lepeophtheirus subni n. sp.; Echthrogalens affinis M. Edw.; Pandarus cranchii Leach.; P. satyrus Dana; P. zystenae n. sp.; Perissopus denlatus Steen., Ltk.; Alebion carchariae Kröy.; Nogagus grandis Steen., Ltk.; N. lunatus Steen., Ltk.; N. Latreillii Leach.; N. curlicaudis Dana; $N$. validus Dana; N. Murray n. sp.; Hessella n. g.; [1'Hessella cylindrica n. sp. è secondo alcuni autori una giovane of di Pennelli. Brady la colloca tra i Dichelesthiidae]; Chondracanthus? macrurus n. sp.; Lernaea hemiramphi (?) Kröyer ; $L$. abyssicola $n$. sp. Quest' ultima specie fu osservata durante il viaguio del Challenger dal $D . *$ v. Villemoes Suhm., sul Ceratias uranoscopus, identico, secondo Günther, col $C$ hobbölli della Groelandiạ. La testa di questa forma è lunga mm. 4, 5 e il corpo coi sacchi oviferi $9 \mathrm{~mm}$.

1898. BrIAN A., 1. Catalogo di Copepodi parassiti dei pesci della Liguria. - Atti della Società Ligustica di Scienze Nat. e Geogr. Vol. IX, tav. I-IV. 
1899. BRIAN A. 2. Di alcuni Crostacei parassiti dei pesci dell'Isola d'Elba. Atti Soc. Lig. di Sc. Nat. e Geogr. Vol. X.

1899. - 3. Crostacei parassiti dei pesci dell' Isola d'Elba (II contributo). Atti Soc. Lig. di Sc. Nat. e Geogr. Vol. X.

1899. - 4. Diphyllogaster Thompsoni n. gen. e n. sp. di Caligidae della Dicerobatis giornae Gunt. -.. Atti Soc. Lig. Sc. Nat. e Geogr. Vol. X, (con tav.).

1901. - 4 a. Caso di anomalia verificatosi su di una Brachiella del Tonno. Bollett. Mus. di Zool. e di Anat. comp. della R. Univ. di Genova, anno rgor, oppure: Cosmos, anno 50, n. 835 ( 5 Genn. Igor). Parigi.

1902. - 5. Note su alcuni Crostacei parassiti dei pesci del Mediterraneo (tav. I). Atti Soc. Lig. Sc. Nat. e Geogr. Vol. XIII.

1902. - 6. Descrizione di un nuovo genere di Crostaceo lerneide: Silvestria truchae n. g. n. sp. - Atti Soc. Lig. Sc. Nat. e Geogr. Vol. XIII.

I903. - 7. Sulla Lophoura Edwardsii Kölliker e sopra alcuni altri Copepodi del golfo di Genova. - Atti Soc. Lig. Sc. Nat. e Geogr. Vol. XIV.

1903. - 8. Sostituzione di nome al nuovo genere di Crostaceo lerneide: Silvestria Brian (= Leptotrachelus Brian)。-Zoolog. Anz. Bd. XXVI, n. 703 vom 29 Juni 1903.

1903. -- 9. Sui Copepodi parassiti dei pesci marini dell'Isola d'Elba (4. ${ }^{\mathrm{a}}$ Nota). Atti Soc. Lig. Sc. Nat, e Geogr. Vol. XIV.

I905. - 10. Sui Copepodi raccolti nel golfo di Napoli da Oronzio G. e da Achille Costa (con 2 tavole). - Annuario del Museo Zoologico della R. Università di Napoli (nuova serie) Vol. I, n. 24, I5 Gennaio I 905 .

1905. - 11. Nouveau copépode parasite (Caligus Remorae n. sp.) - Extrait des Archives de Parasitologie, t. IX, n. 4, p. 564, figg. I-9.

I860. BRÜHL C. B., Mittheilungen aus dem K. K. Zoologischen Institute der Universität Pest. Nr. I. Lernaeocera gasterostei, ein Schmarotzerkrebs aus der Familie der Pennellina. Mit. 2 Taf. Wien.

L'a. scopre la presenza, nelle Lernacocerae, di paia di piedi natatori che non erano prima sospettati: e dà anche una illustrazione delle parti boccali. Le sue osservazioni sono importanti quindi per lo studio delle estremità nei Lerneidi.

I897. Brumpt E., Sur un Copépode nouveau (Saccopsis Alleni, nova species, parasite de Polycirrus aurantiacus Grube); 2 I Juin I897. - C. R. Acad. Sc. Paris.

L'a. descrive un cop. par. di un Polycheta sedentario molto comune nelle cavità delle scorie del fondo del porto di Plymouth. Ne presenta la morfologia. 
I858. BRuzelius R., Om en i Pennatula rubra lefvande parasit. - Oefverss. Vetensk. Akad. Förhandl. XV, p. 18I-185, Taf. 4; oppure Ueber einen in der Pennatula rubra lebenden Schmarotzer. - Wiegmann's Archiv f. Naturgesch. XXV, I, p. 286-290, Taf. 9, 1859.

L'a. descrive la Lamippé rubra Bruz, genere che venne più tardi da Thorell collocato tra i Pacilostoni e vicino ai Chondracanthini, ma Gerstaecker lo esclude assolutamente da questa famiglia, nella quale non sembra doversi comprendere perchè il maschio è presso a poco identico alla femmina, e non pigmeo; quindi resta ancora incerta la sua posizione sistematica.

I869. Buchнolz R., Zur Kenntniss der innerhalb der Ascidien lebenden parasitischen Crustaceen des Mittelmeeres. - Zeitschrift fur Wissenschaftliche Zoologie herausg. von Carl Theodor v. Siebold und A. Kölliker (I $9^{\text {me }}$ vol.) mit taf. V-XI, p. 99-I55. Leipzig, I869.

L'a. descrive specie della fam. Notodelphyidae:

I. Notodelphys mediterranea n. sp. - 2. Notodelphys pusilla n. sp. - 3. Doropygus pullus n sp. - 4. Doropygus gibber Thorell. - 5. Botachus (Thorell) fusiformis n. sp. 6. Notopterophorus (Costa) elongalus Buchholz. - 7. Goniodelphys trigonus n. sp. n. g. 8. Gunenotophorus globularis Costa. - 9. Ascidicola rosea Thor (v. sin.) - Io. Lichomolgus elongutus n. sp.

1833. BURMEISTER H., Beschreibung einiger neuen oder wenig bekannten Schmarotzerkrebse, nebst allgemeinen Bę̧rachtungen über die Gruppe, welcher sie angehören. - Nov. Acta Acad. Leopold. Carolin. XVII, 1, p. 269-336, mit 3 Taf.

L'a. descrive le seguenti specie: Pandarus Carchariae Leach?; Dinematura gracilis n. $\mathrm{sp}$. Chalimus Scombri n. sp.; Bomolochus Belones Burm.; Lernanthropus pupa Burm.; Lernaeocera cyprinacea Blainv.; e termina con osservazioni d'indole generale sopra il gruppo dei Siphonostoma di Latreille, che per quei tempi, non "erano prive $\mathrm{d}^{3}$ importanza. Dà anche un abbozzo di divisione sistematica in famiglie, generi e specie. Le famiglie sono: r. Pennellina; 2. Lernaeoda; 3. Ergasilina; 4. Caligina; 5. Argulina.

Il lavoro è corredato di 4 tavole.

I89i. CANu E., 1. Les copépodes marins du Boulonnais. - V. Les semi-parasites. Bull. Scient. de la France et de la Belgique, T. XXIII, p. 476.

1892. - 2. Su qualche copepodo parassita osservato nel Boulonnais. - C. R. Acad. Sc. Paris, T. II 3 (189I), n. I4, p. 435-437. Extr. in: Revue Scientif., T. 48, n. 16, p. 504-505 (3 n. sp.).

1892. - 3. Les Copepodes du Boulonnais - Travaux du Laboratoire de Zoologie maritime de Wimereux. Amtleteuse (Pas de Calais), Tome VI. Lille, I892.

L'a. tratta con molta competenza dei copepodi liberi e semi-parassiti della fauna marina del Boulonnais e ne dà l'estesa Bibliografia.

L'a. fa menzione, in questo lavoro, della Longipedia coronata, copepode ancestrale (protocopepode). Aggiunge interessanti riflessioni sopra $i$ copepodi in genere e fa allusione anche ai parassiti, specialmente accennando alla Lernaea, al suo sviluppo condensato, alla condensation du diveloppement dei copepodi ascidicoli (ce qui est du principalement da l'accumulation considerable du deutoplasme dans l'ovule): e riporta fatti istruttivi di biologia 
generale, ricordando la progenesi protandrica, la progenesi protoginica ecc. Canu divide i copepodi in monoporodelplaya e diporodelpliva a seconda che hanno una sola apertura sessuale oppure due orifizi sessuali distinti.

L'a. attribuisce a Francesco Redi il merito di avere scoperto il primo copepode normale avendo fatto conoscere il Doropygus della Mentuli (Osservaz. intorno agli animali viventi che si trovano negli animäli viventi. Firenze 1684), mentre si attribuiva questo diritto a Stephan Blankaart per una cattiva figura di Cyclops? pubblicata ad Amsterdam nel 1688. A Leeuwenhoek però spetta il primo documento realmente zoologico su questi animali microscopici.

1857. Carus J. V., 1. Icones zootomicae, I, 'Taf. X.

1885. - 2. Prodromus faunae mediterraneae. Stuttgart.

Sono dall' a. passati in rassegna e brevemente diagnosticati in latino tutti i generi e le specie di copepodi parassiti riscontrati fino al 188 , nel Mediterraneo e nell'Adriatico. Di ciascuna specie é fornita l'indicazione sulla distribuzione geografica e l' habitat. E un compendio molto esatto e l'unico cosi completo finora pubblicato che riguarda ar.che la distribuzione sistematica dei copepodi parassiti dei pesci e d'altri animali nei nostri mari.

I863. Carus.J. V. and Gerstaecker A., Handbuch der Zoologie.

1902. Caullery M. et Mesnil F., Sur le Staurosoma parasiticum Will. Copépode gallicole, parasite d'une Actinie. - C. R. Acad. Sc., Paris, T. I34, n. 22, p. 1314-1317. Extr. Revue Scient. (4), T. 17, n. 25, p. 788-89; oppure C. R. Soc. Biol. Paris, T. 54, n. I9, 6 Juin, p. 629-630.

1858. Chenu D.r, Encyclopédie d'histoire naturelle, Crustacés.

1867. ClaparÈDE ED., Miscellanées zoologiques. Observations sur la Lamippe. Ann. d. Scienc. Natur. 5 sér. Zoologie, Vol. VIII, p. 23-28, pl. 5.

Il gen. Lamippe Bruzelius è stato da Thorell compreso tra i Paciloslomi e in verità in vicinanza dei Chondracanthini. Questo genere possederebbe soltanto un paio di mascelle uncinate (?). Gerstaecker, recentemente, esclude affatto il Lamippe dalla famiglia sopradetta (ed oggi la sua posizione è ancora incerta), dopochè Claparéde riscontrò che i due sessi sono foggiati ugualnente, e che il maschio per conseguenza nulla ha che fare col tipo dei maschi pigmei.

1858. Claus C., 1. Zur Anatomie und Entwickelungsgeschichte der Copepoden. Wiegmann's Arçiv. f. Naturgesch. XXIV, I, p. I-76, Taf. I-3.

1858. - 2. Ueber den Bau und die Entwickelungsgeschichte parasitischer Crustaceen. - Cassel, 1858.

L'a. espone il risultato di esatte ricerche fatte sui seguenti copepodi parassiti, che egli descrive:

Chondracanthus gibbosus Kr. allo stato di larva.e nella forma adulta femminile e maschile; Lernanthropus Kroyeri van Ben. nei due sessi: e dal lato anatomico;

Kroyeria lineata v. Ben. nella forma femminile soltanto.

Osservazioni intorno alla sistematica dei cop. par. si trovano in un capitolo alla fine

r860. - 3. Zur Morphologie det Copepoden. I. Eine Hemmungsbildung von Cyclops. 2. Ueber den Bau von Nicothoë. 3. Ueber die Leibesgliederung und die Mundwerkzeuge der Schmarotzerkrebse. Würzburger Naturwissensch. Zeitschr. I. Bd. p. 20-36, Taf. I. 
1860. Claus C. , 4. Ueber den Bau der Notodelphys ascidicola Allm. - Würzburger Naturw. Zeitschr. I, p. 226-233, Taf. 6.

I86r. - ந. Ueber die Familie der Lernäen: Lernaeocera gobina. - Würzburger Naturwiss, Zeitschr. Bd. II, p. 10-22, Taf. I.

I861. - \%. Ueber den Bau und die Entwickelung von Achteres percarum. Zeitschrift für Wiss. Zool. Bd. XI, p. 287-308, Taf. 23 u. 24.

I862. - \% Untersuchungen über die Organisation und Verwandtschaft der Copepoden. - W.ürzburg. Naturwiss. Zeitschr. Bd. III, p. 5 I-103.

I864. - 8. Beiträge zur Kenntniss der Schmarotzerkrebse. - Zeitschr. f. Wiss. Zool, Bd. XIV, p. 365-382, Taf. 33-36.

$L^{\prime}$ a. premette osservazioni assai istruttive sull'organizzazione interna dei Caligus (C. branchialis Malm, C. gracilis Van Ben. e C. pecioralis Müll.) raccolti in Helgoland, indi passa a descrivere la Lüthenia Asterolermi un nuovo genere di caligide. - Trattando del genere Bomolochus e affini di cui studia la morfologia, descrive le specie B. cornutus, B. soleae, B. (Eucanthus) balistae.

L'a. illustra pure un nuovo notodelfide, ossia lo Sphaeronolus Thorellii, e accompagna la memoria con tre tavole.

I868. - 9. Grundzüge der Zoologie. - Marburg et Leipzig.

1868. - 10. Beobachtungen über Lernaeocera, Peniculus und Lernaea. Ein Beitrag zur Naturgeschichte der Lernaeen. Mit 4 Tafeln. - Schriften der Gesallschaft zur Beförderung der gesammten Naturwiss. zu Marburg, 2. Supplement-Heft.

L'a. descrive la Lernaeocera esocina Herm. e il Peniculus fistula; e studia le metamorfosi della Lernaea branchialis.

1875. - 11. Ueber Sabelliphilus Sarsii und das Männchen desselben. - Zeitschrift f. Wiss. Zool. Bd. XXVI, Taf. ıо. Leipzig.

1875. - 12. Neue Beiträge zur Kenntniss parasitischer copepoden nebst Bemerkungen über das system derselben. - Zeitsehrif̣t für Wiss. Zool. Bd. XXV. 4. mit 3 Taf. Leipzig.

L'a. dopo aver trattato del gen. Hersilia Phil. parla dell' importanza dell' apparato boccale nella divisione delle famiglie naturali, quindi discorre degli Ergasilidi, dei copepodi Nereidicolidi, Ascidicolidi, dei Siphonostomi e in particolare del gen. Lamproglena.

1876. - 13. Die Schalendrüse der Copepoden (9 Nov. 1876). - Sitzungsb. d. K. Akad. d. W. Math. Nat. Cl., Bd. LXXIV. Abth. I.

L'a. tratta di copepodi liberamente viventi, ma incidentalmente accenna anche alle glandole del mantello nel gen. Achiheres, p 720.

1886. - 14. Ueber Lernaeascus nematoxys, eine seither unbekannt gebliebene Lernaee. Anzeiger der K. Akad, der Wiss. Wien. Nr. XXV.

1887. 15. Über Lernaeascus nematoxys Cls. und die Familie der Philichthyden. - Arbeiten des Zool. Institutes zu Wien, Tom. VII, Heft. 2. mit 4 Taf. Wien. 
L'a. descrive il maschio del detto filictide, e la forma giovanile dei due sessi; descrive la femmina adulta, ossia nello stadio di formazione delle uova; tratta in particolar modo della femmina dei generi Philichthys e Sphaerifer, e. infine distingue i caratteri propri a tutta la famiglia, delle forme maschili cosi diverse da quelle femminili. Aggiunge la diagnosi dei gen. Leposphilus di Hesse e del suo Lernaeascus e accompagna $1^{3}$ importantissimo lavoro con 4 magnifiche tavole.

I889. Claus C., 16. Ueber neue od wenig bekannte halbparasit copepoden instes. der Lichomolgidèn u. Ascomyzontiden-Gruppen. Wien. - Arb. Zool. Inst. Wien, T. VIII, p. 39 .

Nematoden

189o. Совв N. A., Neue parasitische Ar. Abbild. - Arch. f. Naturg. 55. Jahrg., I Bd., 2 Hft., p. I49-I5I. v. Kükenthal. Fauna Spitzbergens.

1780 -? Cordiner, Lernaea salmonea Linn. - Antiq. and Sc. of N. Scot. 7, 8, pl. VI, fig. 2 .

1865. Cornalia E., 1. Sulla Lophoura Edwardsii di Kölliker. Osservazioni zoologiche e anatomiche. - Atti della Società Italiana di Scienze Naturali. Vol. IX, p. I-IO, tav. I.

I'a. completa la descrizione che ci ha lasciato Kölliker su questo interessantissimo parassita e l'accompagna con buone figure.

1874. - 2. Sulla Taphrobia pilchardi n. g. di crostaceo parassita. - Atti Soc. Ital. Sc. Nat., Vol. XVIII, fasc. II, p. I97, tav. VI.

r84o. Costa O. G., Fauna del Regno di Napoli. Entomostraca (i 840 ) 1852.

Nel Catalogo dei crostacei del Costa, pubblicato nel I 840 , separatamente dall' opera sui crostacei-colle tavole, che comparve ulteriormente, si trovano citate sei specie di copepodi, che sono: Cyclops marinus (Euchäta); Edwara'sia (Sapplirinu) fulgens; Cecrops Latreillii; Gunenolophorus olobularis; Noloplerophorus elongatus; N. elatus.

Di tutte queste forme citate, il Costa descrisse soltanto l'Edwardsia fulgens, corredando di figure la descrizione. Pure figurati, ma non descritti appariscono soltanto nel testo il Gunenotoplorus globularis, Notopterophorus elongalus, $N$. elafus, ma non è stata riportata nè la figura, nè alcun cenno sulle rimanenti forme. In una tavola segnata col numero 3 in fondo all'opera, l'a. ha rappresentato un copepode parassita senza nominarlo. La figura è inesatta, tuttavia ritengo che il copep. parass. disegnato possa essere una Nemesis (forse N. robusta); ma evidentemente si osserva. che il disegnatore è incorso in un errore nel rappresentare un individuo maschile, al quale egli ha appiccicato i tubi oviferi esterni, propri della femmina.

I798. Cuvier G. L., 1. Tableau élémentaire de l'histoire naturelle. Paris.

- 18r7. - 2. Régne Animal. Paris. Première edit. Tom. III.

I829. - 3. Régne Animal. Paris. (Edizione riveduta). Tom. III.

I850. Dana J. D., 1. On the eyes of Sapphirina, Corycaeus etc. - Silliman's Americ. Journ. of Science, 2 sér., IX, p. I33. 
I852. DANA J. D., 2. United States Exploring Expedition during the years r838-1842, under the command of Charles Wilkes. U. S. N. XIII. Crustacea, I 852. (Atlas Crustacea, Philadelphia I855).

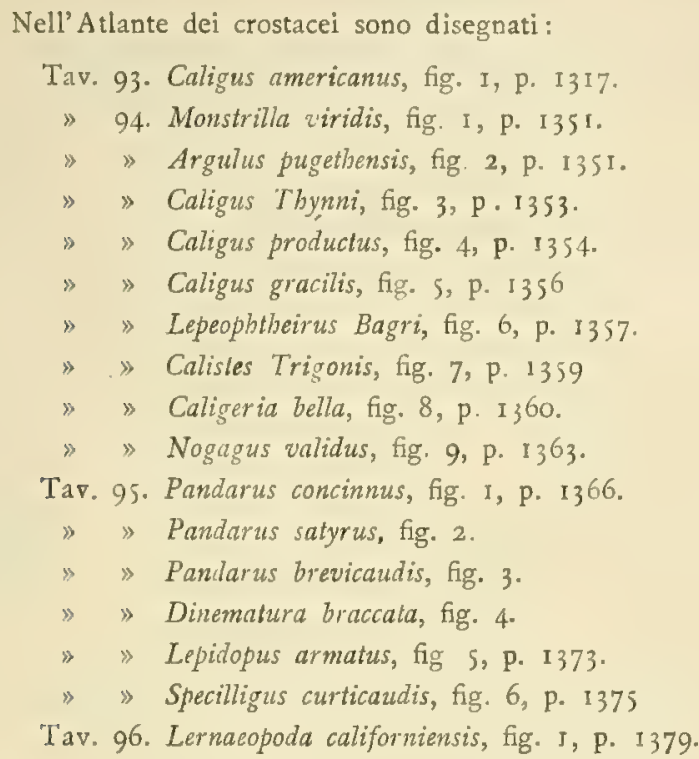

I8II. Delaroche F., Sur deux animaux vivants sur les branchies des poissons. Nouv. Bull. d. 1. Soc. Philomat. II, p. 270-272.

Sopra Chondracanthus Zei e Chondr. Thynni.

I880. Della Valle A., 1. Sui Coriceidi parassiti e sull' anatomia del gen. Lichomolgus. - Roma, 2 tav.

Dopo una prefazione con dissertazione sui coriceidi parassiti, l' autore passa a descrivere: Lichomolgus Sarsii Claparede, L. Actiniae n. sp., L. Pteroidis n. sp., L. Chromodoridis n. sp.; n. g. Anlbessius, A. Solecurli n. sp., A. Pleurobrancheae n. sp.

I883. - 2. Sui Copepodi che vivono nelle Ascidie composte del golfo di Napoli. Relazione del prof. Todaro in: Atti R. Accad. Lincei (3). Transunti Vol. 7, Fasc. 9, p. I 80.

L'a. descrive Enterocola fulgens van Beneden e Kossmechthrus notopus Della Valle, (I tavola).

I825. DeSmaRest A. G., Considerations générales sur la classe des crustacés. Paris.

Vi é soltanto un sunto sui crostacei tolto da Blainville (Dict. d. sc. nat.). •

1763. Ellis. Philos. Transact. Vol. 53, p. 433.

Intorno alla Pennalula filosa e sagitta.

rgor. Embleton A. L., Goidelia japonica (n. gen. n. sp.). A New Entozoic Copepod from Japan, associated with an Infusorian (Trichodina). With 2 pls. - Journ. Linn. Soc. London, Zool. Vol. 28, No 181, p. 2II, 228, 229 - in Abstr. Journ. R. Micr. Soc. London, I goI. P. 3 , p. 277 . 
17... FABRICIUS Chr., Reise nach Norwegen, p. 284.

Sopra la Lernaea Pinnarun.

I780. Fabricius OtTo, 1. Fauna gronlandica, p. 335 und ff.

1794. - 2. Heltens Gjelle-orm, Lernaea Lavareti. - Skrivt. Naturhist. Selsk. Kjöbenhavn III, 2, p. 2I-25.

I860. Fischer S., Beiträge zur Kenntniss der Entomostraceen - Abhandl. Math. Phys. Classe Akad. München, S. Bd., 3 Abthlg., p. 645-68o, Taf. 20-22.

L'a. dà la descrizione e la figura di Lepeophtheirus exsculptus Fischer, la cui patria è ignota.

1847. Frex H. und Leuckarit R., Beiträge zur Kenntniss wirbelloser Thiere, 1847 , p. 165 .

Gli autori rilevano che il Caligus leptochilus è identico al C. rapax M. E. ?; descrivono il Chalimus fissato sull'esterno tegumento di Caligus curtus; Nogagus gracilis; Pandarus bicolor; P. lividus n. sp., tutte specie dell' Helgoland.

Igor. FRIC A, und VAVRA V., Untersuchungen über die Fauna der gewässer Böhmens, V. Prag, igor. - Archiv der Naturw. Landesdurchforschung von Böhmen, XI Band, No 3.

Igor. GAdD PEHR., 1. Nagra förut obeskrifna, parasitiskt lefvande Copepoder. Meddel. Soc. Fauna Flora Fenn. 27 Hft., p. 98.

L'a. descrive le n. sp. Ergasilus biunciratus, Achlheres sandrae, Lernaeopoda exlumescens.

rgo4. - 2. Parasit-Copepoder i Finland. - Acta Societatis pro Fauna et Flora Fennica, XXVI, N. 8.

1895. Garbini A., Appunti di Carcinologia veronese. - Mem. dell'Acc. di Verona, Vol. LXXI, serie III, fasc. I. Verona.

L'autore enumera 8 specie di crost. parassiti di pesci delle acque dolei:

Ergasilus Sieboldii v. Nordm. [sul Cyprinus; branchie].

Diclselestium sturionis Herm. [Acipenser sturio; branchie].

Lamproglena pulchella Nordns. [Alburnus e Scardinius; branchie].

Lernaeocera esocina Burm. [Esox; branchie].

Lernaeocera cyprinacea L. [Cyprinus; branchie, muscoli].

Anchorella emarginata [Alosa; branchie].

Aclitheres percarum Nordm. [Perca; gola, archi branchiali].

Argulus foliaceus L. [Cyprinus e Gasterosteus; pelle].

1870. Gegenbaur C., Grundzüge der vergleichenden Anatomie. Leipzig.

1853-54. GeRstaecker A. D. \% 1. Ueber eine neue und eine weniger gekannte Siphonostomen-Gattung, und Beschreibung zweier neuer Siphonostomen Gattungen. - Wiegmann's Archiv f. Naturgesch. XIX, I, p. 58-70, Taf. 3 u. 4; XX, I, p. I85-195, Taf. 7, I853-54.

L'a. descrive in questi due lavori prima l'Elytrophora brachyptera e poi Lonclidium aculeatum (Kröyeria van B.), Gangliopus pyriformis e Nogagus angustatus. 
1870-71. Gerstaecker D." A. 2. D.r H. G. Bronn's Klassen und Ordnungen des Thier-Reichs. V. Band, Gliederfüssler. Lieferung II-I6. Leipzig u. Heidelberg.

I887. Giard A., 1. Sur un Copépode (Cancerilla tubulata Dalyell), parasite de 1'Amphiura squamata Delle Chiaje. - C. R. Acad. Sc., Paris, T. I04, p. II8y, séance du 25 Avril I887.

1889. - 2. Sur l'association de Pennella Orthagorisci Parceval et de Conchoderma virgatum Spengl. -- Le Naturaliste (2) II. Ann. N. ${ }^{\circ}$ 50, p. 82.

$\mathrm{Si}$ sono osservati Conchoderma (cirripede) sia nella regione toracica, sia in vicinanza delle aperture genitali di Pennella di Balaenoptera rostrata Fabr. Per esempio P. Mayer ha osservato Pennelle ( $P$. flosa I. ?) di Xiphias gladius che portavano Conchoderma nella loro parte posteriore, e Giard ha segnalato l'associazione di C. virgatum con Pennella Orlhagorisci Parceval.

I889. - 3. Sur le Peroderma cylindricum Heller, Copepode parasitaire de la Sardine. - C. R. Acad. Sc. Paris, T. 107, n. 23, p. 929-93I. Revue Scientif. (3), T. 42, n. 24, p. 777. I889.

I895. - 4. Sur l'éthologie du genre Thaumaleus Kröyer (famille des Monstrillidae). - C. R. Acad. Sc. Paris, T. I20, p. 937-940.

I896. - 5. Sur le parasitisme des Monstrillidés. - C. R. Acad. Sc., Paris, T. I 3 , n. 20, p. 836-839. Extr. Revue Scient. (4), T. 6, n. 6, n. 23, p. 726. Académie des sciences. Séance du I6 Nov. I896.

1897. - 6. Sur le parasitisme placentaire des Monstrillidae. C. R. Soc. Biol. Paris (I0), T. 4, N. 5, p. I $37-\mathrm{I} 38$.

I900. - \%. Sur un type oublié de la famille des Monstrillidae (Thaumatoessa armoricana Hesse) et sur un cas nouveau de parasitisme chez les Monstrilla. - Bull. Soc. Entom. France, N. 20, p. 395-397.

(n. g. Thaumatohessia).

i889. Giard A. et Bonnier J., I. Note sur 1'A spidoecia Normanni et sur la famille des Choniostomatidae. - Bull. Scient. de la France et de la Belgique, T. XX, p. 34I-72.

1893. - 2. Sur deux types nouveaux de Choniostomatidae des côtes de France: Sphaeronella microcephala G. et B., et Salenskia tuberosa G. et B. - C. R. Acad. 'Sc., Paris, 25 Sept. 1893.

1894. - 3. Contributions à l'étude des Epicarides - Sur les Epicarides parasites des Arthrostracés et sur quelques Copépodes symbiotes de ces Epicarides. - Bull. Scient. de la France et de la Belg. T. XXV, p. 417-493, ta.b. 5-13.

1888. Giesbrecht W., I. Berichte d. Commiss. z. Unters. d. deutschen Meere, p. 88.

1897. - System der Ascomyzontiden, einer semiparasitischen Copepoden Familie. - Zool. Anz., 20 Bd. N. 521, p. 9-I4 - N. 522, p. 17-24. 
1897. Giesbrecht W., 3. Notizen zur Systematik der Copepoden. - Zool. Anz. 20 Bd. N. 536, p. 253-255. r897 - Ausz. von F. Zschokke. Zool. Centralbl. 4 Ihg. N. 18, p. $627-628$.

1899. - 4. Die Asterocheriden des golfes von Neapel und der Angrenzenden Meeres - Abschnitte: (A. u. d. Tit.: Fauna und Flora des golfes von Neapel, 25 Monogr.) Mit I I Taf. Berlin. (2 Tit., 2 I 7 p.).

1751. GisLer, Laxlusen. - K. Vet. Akad. Hand1.

1883. Gissler C. F., A new Parasitic Copepod Crustacean. - From the American Naturalist, Vol. 17, p. 885-886. August, I883, with figg.

L'a. descrive Caligus pacificus no. sp.

1784. GOEZE J. E., Von den Fischlernäen. - Leipziger Magazin zur Naturkunde, p. 39-49. Mit I Tafel.

I905. Goggio E., Intorno al gen. Clavella (Hatschekia Poche). Estratto dall'Archivio Zoologico. Napoli, I905, Vol. 2, Fasc. 2, da pag. 215 a pag. 225 , Tav. I3.

L'a. descrive nuove specie: Hatschekia Cernae sp. D. - Hatschekia Richiardii sp. n.

I842. GoODSIR H., 1. On a new genus and on six new species of Crustacea, with observations on the development of the egg and on the metamorphoses of Caligus, Carcinus and Pagurus. - Edinburgh New Philosoph. Journ. XXXIII, p. I74-192, c. tab. 2.

1842. - 2. Note sur le développement des œufs du Caligus et sur les métamorphoses que ce Crustacé éprouve. - Ann. Scienc. Natur. 2 sér., Zool. Vol. XVIII, p. I8I-I84.

L'a. è il primo ad aver fatto conoscere le uovadei caligidi e i primi stadi đi sviluppo di esse (Nauplins) in una comune specie di Caligus trovata in Scotland. Descrive anche gli organi riproduttori della femmina adulta.

I784. GöTZE J. E., Von den Fischlernäen. - Leipziger Magazin zur Naturkunde, p. 39-49, mit I Tafel.

I887. Gourret P., 1. Su qualche crostaceo parassita dei Phallusii. - C. R. Acad. Sc. Paris, T. I04, N. 3, p. $185-187$ - in: Abstr. in Journ. R. Microsc. Soc. London I887. P. 3, p. 392.

I888. - 2. Études zoologiques sur quelques Crustacès parasites des Ascidies. Paris.

Ig02. GRAefFe, Übersicht der fauna des Golfes von Triest. v. Crustacea S. I6. - Arbeiten d. Zool. Inst. Wien, Bd. XIII.

I827. GRant R. E., 1. On the structure and characters of Lernaea elongata from the artic. Seas - Edinburgh. Journ. of Science VII, p. I47-I54. 
1829. GRANT R. E., 2. Sur la structure et les caractères de la Lernaea elongata. - . Férussac Bull. Scienc. Natur., XVII, p. 3i 2-3iz.

Questo lavoro e il precedente uguale, contengono la più completa e la migliore descrizione di una Lernaea fino al 1832.

I86I. GRuBE, Ausflug nach Triest und dem Quarnero. - Beiträge zur Kenntniss der Thierwelt. Berlin.

L'a. cita nell' elenco degli animali osservati nell'Adriatico, tre sole specie dei nostri crostacei succhiatori; il Lernanthropus Kröyeri, l'Anchorella uncinata e una specie nuova di Chondracanthus.

1840. GUUÉRIN, Iconographie du Règne animal. Crustacés. Zoophytes.

L'a. illustra alcune forme note a' suoi tempi di copepodi parassiti: Caligus piscinus Guérin (oggi Lepeophtheirus Thompsoni Baird); Cecrops Latreillii Leach., tav. 35, fig. 8; Anthosoma crassum Abild., tav. 35, fig. 9; Dichelesthium Sturionis Herm., tav. 35, fig. Io; Nemesis Lammae Risso (N. mediterranea), tav. 35, fig. II; Chondracanthus Triglae Nordm., tav: IX, fig. 8 (Medesicasle); Ch. Zii, tav. IX, fig. 9; Brachiella Thynni Cuv., tav. IX, fig. 6.

I863. HAECKEL E., Beiträge zur Kenntniss der Corycaeiden. - Jenaische Zeitschr. für Mediz. u. Naturwiss.

I863. HANCOCK A. and NORMAN A., On Splanchnotrophus an undescribed genus of Crustacea parasitic in Nudibranchiate Mollusca. - Transact. of the Linnean Soc. f. London, Vol. XXIV, p. 49-60, p1. I5, I6.

Questo genere di copepode parassita è incluso nella famiglia Chondracanthidae.

I892. HANSEN H. J., 1. Rhizhorhina Ampeliscae n. gen. n. sp. En ny til Herpyllobiidae n. fam. horende copepod, snyltende paa Amp. laevigata Lillb. - Entomol. Medd., III. Kjobenhavn.

L'a. descrive il detto copepode appartenente alla fam. Herpyllobizidae, che vive parassita sull'Ampelisca luevigatu Lillj; e la Salenskya tuberosa G. et B., parassita dell'A. Spinipes Boeck. Questi crostacei inviano nel corpo del loro ospite una specie di tentacoli ramificati e rigonfi.

1897. - 2. The Choniostomatidae. A family of Copepoda, Parasites on Crustacea Malacostraca. With 13 pls. - Copenhagen. Fred Host \& Sen. (206 p.)

Secondo J. Richard, ancora poco tempo fa non si conosceva che qualche raro copep. par. d'altri crostacei, mentre che un gran numero di specie di quest'ordine erano conosciute come parassite d'altri animali, in particolare dei pesci. Hansen ha fatto conoscere, dopo, più di quaranta specie d' una famiglia nuova (Choniostomatidae) di cui tutti i membri sono parassiti dei malacostraci. Uno solo (Aspidoecia) vive sul lato o sul dorso del suo ospite, qualcheduno vive nella camera branchiale, ma il più gran numero si trova nel marsupium dell' animale infestato. In generale won si trova che una sola specie sul medesimo ospite $\mathrm{c}$ il più spesso un of e $\%$; tuttavia si conoscono specie che si trovano in ispecie diverse di crostacei. Le dimensioni di questi copepodi parassiti

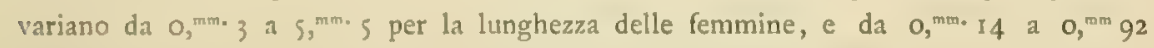
per quella dci maschi. Il rostro, bene sviluppato nei due sessi, ha l'aspetto d'una ventosa in fondo della quale si vede la bocea e le punte delle mandibole. Si sa che l'Aspiduciu fora il suo ospite e ne succhia il sangue; c' è luogo a credere che lo stesso 
si verifichi per gli altri membri della medesima famiglia, a causa della similitudine che si osserva nella struttura dei pezzi boccali. Gli ovisacchi sono deposti separatamente; Hansen ne ha trovato fino a 28 deposti da una medesima fermina.

Giard e Bonnier hanno mostrato che i crostacei parassiti dei diversi gruppi causano la castrazione parassitaria nel loro ospite. E anche assai generalmente il caso dei copepodi di cui é questione qui. Salvo tre, che sono parassiti degli isopodi appartenenti ai generi Ianira e Munnopsis, e cinque, parassiti di cumacei (G. Diaslylis, Endorella e Iphinoe). tutte le specie di Sphaeronella sono parassite di amfipodi cosi come i Stenothocheres. Gli Homaoscelis si trovano nei cumacei, i Choniostoma nei caridi, i Mysidion e gli Aspidoecia sui mysidi.

La lista delle specie di questa grande famiglia di cop. par. è numerosissima ( 46 specie).

I902. HANSEN H. J., 3. Echinocheres globosus n. gen., n. sp., a Copepod parasitic in spines of an Echinothurid. With I pl. - Vid. Meddel. Nat. Foren. Kjobenh., p. 437-448, 449 .

I87 I. HARtMANN R., 1. Über d. v. Poren durchsetzte Chitinskelet d. Caliopus Cecrops u. gewisser Lernaeoceren. - Sitzber. d. Ges. Naturforsch. Freunde. Berlin (Oct. I870) I87x, p. 60-6I.

I870. - 2. Beitrage zur anat. Kenntniss der Schmarotzerkrebse. - Archiv fur Anat. u Phys., Ihg. I870.

r. -- Bomolochus Belones Burm., p. I r6-158, Tav. III-IV.

2. - Lernaeocera Barnimii mihi, p. 726-752. Tav. XVII-XVIII.

Sono due importanti monografie nelle quali i detti copepodi parassiti sono studiati in modo quasi esauriente sotto il punto di vista morfologico e anatomico, almeno per lo stadio adulto.

I888. HARTOG M. M., The morphology of Cyclops and the relations of the Copepoda. - Trans. Linn. Soc. London, V. p. I-46.

1893. Hecht E., Note sur un n. Copep. par. des Nudibranches. - Arch. Zool. Expérim. (3) T. I, N. I, p. XIII-XVI.

L'a. descrive lo Splanchnotrophus angulatus Hecht. parassita negli eolidi (Aeolis papillosa L. e Aeolis glauca A. et $\mathrm{H}$.) e trovato a Roscoff.

1879. HeIder C., 1. Die gattung Lernanthropus. - Arbeit. d. Zool. Instit. Wien. u. Triest. T. II. Hft. 3, p. 269-368, mit 5 Taf. - Auch separ.: Wien, Hölder, I879.

E una interessantissima monografia del genere Lernanthropus colla descrizione di tutte le specie che erano note fino al 1879 . L' autore scopre e descrive (in pari tempo di Van Beneden) in questi animali, un apparato di circolazione, ossia un sistema vascolare in cui scorre il sangue o meglio un liquido hematique come vuole Van Beneden, che avrebbe l'ufficio dei globuli rossi nei vertebrati.

I880. - 2. Abwehr (gegen van Bened.). - Zoolog. Anzeiger, 3 Ihg. I880, p. 93-94.

I865. HeLleR C., 1. Crustaceen der Novara-Expedition.

E un voluminoso lavoro interessantissimo sotto il rapporto dclla specigrafia e indispensabile agli studiosi di copepodi parassiti, essendo descritte molte specie nuove. I generi trattati dall' a. sono in numero di 36 e le specie di 64 . 
I 866. HeLLer C., 2. Carcinologische Beiträge zur fauna des Adriatischen Meeres. Aus den Verhandlungen d. K. K. Zoolng.-Botanischen Gesellschaft in Wien (1866) besonders abgedruckt.

E un elenco di crostacei, fra cui non pochi copepodi parassiti viventi nell'Adriatico.

I780. Herbst J. F. W., 1. Beschreibung einer sehr sonderbaren Seelaus vom Hamorfisch. - Schrift. d. Gesellsch. Naturf. Freunde zu Berlin, I, p. 56-67, mit I Taf.

L'a. descrive con gran numero di dettagli il caligide col nome di Seelaus vom Hemorfisch, che corrisponde alla Dinemoura Lamnae di Baird e alla Dinematura producta di Müller.

I78I. - 2. Beschreibung der Flinderlaus. Schrift, d. Gesellsch. Naturf, Freunde zu Berlin, III, p. 94-ro2, mit. Taf.

1903. Herdman W. A., (vedi I. Thompson and A. Scott). - Report to the government of Ceylon on the Pearl oyster fisheries of the gulf of Manaar. Published by the Royal Society, I903. - Supplementary Report VII. On the Copepoda by Isaac. C. Thompson, F. L. S., and Andrew Scott, A. L. S.

1783. Hermann, Helmintologische bemerkungen. - Naturforscher, n. I9. (Üeb. Lernaea squamicola und L. Lotae).

I884. HERRTCK C. L., A Final Raport on the Crustacea of Minnesota (Cladocera a. Copepoda) Minneapolis.

I838. HERRIK anci DANA, United States exploring Expedition. - Silliman's Journal, Vol. XXXIV.

1858. Hesse E, 1. Mémoire sur les moyens à l'aide des quels certains Crustacés parasites assurent la conservation de leur espèce. - Ann. d. Scienc. Nat., 4 sér. Zoolog., Vol. IX, p. I20-I25.

L'autore descrive il cordone frontale con cui i giovani Pandarini e Chondracanthus durante lo stato di Chalinus si fissano per un certo periodo sul corpo della madre.

1860. - 2. Embryons de Caliges (Caligus) et de Trébies (Trebius) in C. R. Acad. Sc., Paris, T. 5I, p. 715.

1862-68. - 3. Recherches sur les Crustacés rares ou nouveaux des côtes de France. Ann. d. Scienc. Nat. 4 sér., Zoolog., Vol. XVII, p. 343-355, pl. 18; idem Vol, XX, p. IоI-I32, pl. I.

Idem 5 sér., Zoolog. Vol. III, p. $221-226$.

\begin{tabular}{|c|c|c|c|c|}
\hline 》 & $\gg$ & $\gg$ & $\gg$ & IV, p. 229-257, pl. 6 et 7 \\
\hline$\gg$ & » & $\gg$ & $»$ & VI, p. $5 \mathrm{I}-86$, pl. 4 \\
\hline$\gg$ & $\gg$ & $\gg$ & $»$ & VII, p. I 99-2I 5, pl. 4 . \\
\hline so & $\gg$ & $\gg$ & $\gg$ & XI, p. 53-6r. \\
\hline
\end{tabular}


I864. Hesse E., 4. Observations sur des Crustacés rares ou nouveaux des côtes de France. - Ann. Scienc. Nat., 5 sér., Zoolog., Vol. I, art. 3 , p. $333-356, \mathrm{pl}$. I I et I 2 .

L'a, descrive i seguenti copepodi par, delle ascidie:

Notopterophorus papilio Hesse, Polycliniophile corisoformis Hesse, Botrylloplitus ruber Hesse, B. virescens Hesse, Ichnograde ruber Hesse, Podolabis fulvus Hesse, P. albidus Hesse, Ophioseite cardiocéphale Hesse.

1877. - 5. Description des mâles des Lernanthropes de Gisler et de Kröyer. - Rev. Sc. Natur. VI.

1877. - 6. Remarques sur le genre Chalime. - Ann. Scienc. Nat., 6 sér., Zoolog. Vol. V, art. Io.

1879. - \%. Description des Crustacés rares ou nouveaux des côtes de France. - Ann. d. Scienc. Nat. 6 sér., Zoolog., Vol. VIII, art. 29.

L'a. descrive 7 specie n. di Cycnus che in un mio lavoro ho dimostrato appartenere al gen. Clavella oggi Halschekia; e 3 specie di Krōyeria:

Cycnus Crenilabri Hesse; C. Labri mixli; C. Labri Donovaini; C. Acantbolabri exoleli; C. Labri trimaculati; C. Pagelli Bogneravei; C. Canthari grisei; Krōyeria Scyllii Caniculae Hesse; $K$. Carchariae-glauci; $K$. Acanthias vulgaris.

1879. - 8. Description d'un nouveau Crustacé parasite appartenant à la sousclasse des crustacés suceurs, de l'ordre des Lernéides, formant la nouvelle famille de Lernèopalmiens et le nouveau genre des Stylophores. - Ann. d. Scienc. Nat., 6 sér., Zoolog., Vol. VIII, (I879), art. 15, p. $3 \mathrm{I}$.

La femmina di questo cop. par. descritto da Hesse, è lunga 5 cent. ed è stata trovata nella cavita nasale della Raja rostrata.

I880. - 9. Description de deux Crustacès nouveaux male et femelle du genre Dinemoure, dècrits et peints sur les individus vivants. - Extrait de la Revue des Sciences Naturelles. Juin I880.

L'a. descrive la Dinematura Musteli Jaevis n. sp.

I883. - 10. Crustacès rares ou nouveaux des côtes de France. (Descrip. de plusieurs Crustacès parasites de l'ordre des Siphonostomiens) Ann. d. Scienc. Nat., 6 sér., Zoolog., Vol. XV, art. 33, 48 pagg. pl. 4-6.

L'a. descrive la forma giovanile di Nogagus spinacis acantline n. sp.; dà il suo contributo alla conoscenza della fisiologia e biologia di alcuni caligidi: Cecrops Acanthiivulgaris n.; Lepimacrus n.; L. Tourdainii sopra Lamma cornubica; Pandarus Carchariae glauci n.; P. Musteli-laevis n.; P. Spinacii acanthias n.; P. unicolor.

1884. - 11. Crustacès rares ou nouveaux des côtes de France. Ann. d. Scienc. Nat., 6 sér., Vol. XVI, art. 34, p. I-I 8, pl. I 2-I 4.

L'a. descrive Eudactylina Carchariae-glanci n.; E. Musteli-laevis n.; E. Squatinaeangeli n.; Kröyeria Galei-vulgaris n. ; Pagodina Carchariae-glauci n. 
1886. Hesse E., 12. Descript. de deux Crustacès parasites de l'ordre des Siphonostomes. Ann. d. Scienc. Nat., $7^{\text {e }}$ sér., Zoolog., Vol. V, art: 37, p. $340-362$, pl. I $4-15$.

L'a. descrive Cecrops Latreilizi e Laemargus muricatus.

1857. Heven J. Van der, Note sur les genres Cecrops et Laemargus. - Mémoires d'Entomol. d. 1a Soc. Entom. des Pays-bas, I, p. 67-87.

L'a. descrive il Cecrops Latreillii Leach 우 e $\delta^{7}$ e il Laenargvs muricatıs Kröyer \% e $\sigma^{\circ}$.

185 ז. Hope F. G., Catalogo dei Crostacei ital. è di molti altri del Mediterraneo. Napoli.

I802. Holten H. S., Lernaea Merlucii og Exocœti, to nye Arter. - Skrivt. Naturhist. Selsk. Kjöbenhavn, V, 2, p. I35-137.

1879. HoRst R., 1. Uber zwei neue Schmarotzerkrebse M. 2 Taf. - Tijdschr. Nederl. Dierkund. Vereenig. (Haag) 4 Deel. p. 5 I-55.

L'a. descrive Lernaeenicus Gempyli (von einem Fische) e un'altra specie di cop. parassita della Polynoë rarispina.

1897. - 2. Philorthagoriscus serratus Kr. (Dinematura serrata Kr.) With I pl. Notes Leyden Mus., Vol. I9, N. ${ }^{1 / 2}$. Note XIV, p. I37-I44.

L'a. stabilisce un n. g. per la Dinèmatura serrata di Kröyer.

1900. Jensen Soren, Nogle Oplysninger om Rhizorhina Ampeliscae H. J. H., Herpyllobius arcticus Stp.-Ltk. og Fam. Herpyllobiidae. Med 2 Tavl. - Overs. K. Dansk. Vid. Selsk. Forhdlgr. n. I, p. 6I-ro4, 105-106.

1824. Johnston G., 1. Notice respecting the genus Caligus of Leach - Edinburgh Philosoph. Journ. X, p. 292-294.

1835. - 2. Lernaea uncinata. - London's Magaz. of Nat. Hist. VIII, p. 565-566.

I835. - 3. Pandarus alatus and lamnae (ebenda VIII, p. 202-205).

1836. - 4. Chondracanthus lophii, nov. spec. (ebenda IX, p. 8I-83).

1888. Joubin L., Note (contenue dans un pli cacheté) sur les ravages causés chez les Sardines par un Crustacé parasite. - C. R. Acad. Sc., Paris, I 9 Novembre 1888, p. $842-844$.

1889. - Sur un Copépode parasite des Sardines (Lernaeascus)? - C. R. Acad. Sic., Paris, T. 107, n. 27 , p. II $77^{-1178 .}$

1892. KANE W. F. DE Wismes, On a new species of Lernaeopoda (bidiscalis) from the West Coast of Ireland, and Polperro, Cornwall. - With 2 pl. Proc. R. Ir. Acad. (3) Vol. 2, n. 2, p. 203-21. - Abstr. : Journ. R. Micr. Soc. London 1892. P. 4, p. 480. 
I863. KEFERSTEIN W., Ueber einen neuen Schmarotzerkrebs, Nereicola ovata Keferst. - Zeitschr. für Wissensch. Zoolog., XII, p. 46r-463, Taf. 42 .

L'a. descrive Nereicola ovata sp. n. parassita sulla pelle di Nereis Beaucoudrayi Aud.

1879. Kerschner, Ueber zwei neue Notodelphyiden. - Denksch. d. K. K. Akad. Wiss. Wien. Bd. XLI, p. 9 e Io.

Questo autore ha notato per primo l' esistenza dell' ovario impari nei Notodelpijys, Doropygus ecc, e indicato il modo di sviluppo degli ovuli a modo di rosarii intrecciati in seno degli ovidotti.

I887. KIRK T. W., On a curious Parasite (Anthosoma Smithii Leach.) from the Porbeagle Shark (Lamna cornubica). Tiith. I pl. - Trans. N. Zealand. Inst. Vol. 20 (3), p. 3i-33.

I859. KNER R., 1. Uber männchen und weibchen von Euryphorus Nordmanni M. Edw. - Sitzungsber. d. Wiener Akad.d. Wissensch., XXXIV, p. 268-274, mit I Taf. Wien.

I890. KeHLeR R., Les Crustacés parasites des Ascidies. Avec 12 figg. - Le Naturaliste, Ann. I2, n. 78, p. I3I-I34; n. 79, p. I37-I38.

1835. Kollar V., Beiträge zur Kenntniss lernaeenartigen Crustaceen. - Annalen des Wiener Museums für Naturgeschichte, I, I, p. 72-92, mit 2 Taf.

L'a. descrive e figura i seguenti copep. paras.: Tracheliastes stellifer Kllr., Tr. maculatus Kllr., e fa osservazioni sul Tr. polycolpus Nordm., quindi descrive e figura Basanistes Huchonis Nordm. e ne studia la larva e lo sviluppo.

1853. KöLLIKER A., Bericht über einige im Herbst 1852 in Messina angestellte vergleichend anatomische Untersuchungen. Gatt. Lophoura. Zeitschr. f. Wissensch. Zoologie, IV, p. 359.

L'a. descrive la nuova forma femminile di un copepode par. Lophoura Edwardsii, trovata infissa nella carne del Lepidoleprus ccelorbynclus e che sta vicino allo Sphyrion laeve di Cuv.

I877. Koren J. a Danielssen D. C., Fin ny art af slaegten Pennella. -- Faun. litt. Norweg. $3 \mathrm{Hft} .$, p. $157-\mathrm{I}_{3}$.

Gli autori descrivono una nuova specie del gen. Pennella.

1890. KorSChelt E. und HEIDER K., Entwickelungsgeschichte der wirbellosen Thiere, Jena.

1877. Kossmann R., Zoologische Ergebnisse einer Reise in die Küsten gebiete des Rothen Meeres. - Entomostraca (I theil Lichomolgidae). Leipzig, p. 24 con VI tav. (Parte IV).

Dei Copepodi parassiti del mar Rosso fino al 1877 , secondo l'a., solo erano note due specie non denominate di Caligus, Bomolochus parvulus, Lamproglena Lichiae e I. Hemprichi. 
I837-38. KRöYER H., I. Om Snyltekrebsene, isaer med Hensyn til den danske Fauna.

I. Om Lernaeerne i Almindelighed.

II. Systematisk Oversigt af Snyltekrebsene.

III. Formbeskrivelser.

Naturhistorisk Tidsskrift, I. p. I72-208, p. 252-304, p. 476-505, p. 605-628, tab. $2-3$ u. 5-6. - II. p. 8-52, p. I $3 I-I 57$, tab. I u. 3 . Kjöbenhavn.

L'a. dà molte figure che in parte sono troppo poco ingrandite. I sei fascicoli molto istruttivi contengono, tra altri generi, alcuni nuovi fondati dallo stesso autore: Aethon quadratus trovato sulle branchie di un Serranus; Indie occidentali. - Selius bilobus dell'Aphrodite punctata e Tucca impressus tolto dalle pinne pettorali di Diodon hystrix; Indie occidentali; (genere che fu piu tardi, nel 1865 , studiato di nuovo da Nordmann).

1863. - 2. Bidrag till Kundskab om Snyltekrebsene. - Naturhistorisk Tidsskrift, 3. Raekke, II. Bind., p. 75-426, tab. I-18. - Separat: Kjöbenhavn, 1863

È un lavoro importantissimo con diagnosi latine. Disgraziatamente il testo è scritto in norvegese, lingua poco accessibile per noi italiani.

L'a. descrive una moltitudine di specie nuove: 18 specie n. di Caligus; i4 sp. n. di Lepeophtheirus; alcuni Dinematura, Lernanthropus, Bomolochus, Ergasilus, molti Chondracanthus, moltissime nuove Anchorellae, per tacere di una quantità di nuovi altri generi e specie appartenenti a famiglie diverse e che troppo lungo sarebbe qui enumerare. In tutto II specie di copepodi parassiti nuovi o poco noti e generi 37 , senza contare 5 forme di argulidi. Il lavoro è accompagnato da 18 tavole con figure che forse avrebbero avuto maggior pregio, un po' più ingrandite.

I870. KRöYER og SchiöDte, Herpyllobius og Silenium. Kjöbenhavn.

1877. KURZ W, 1. Studien über die Familie der Lernaeopodiden. - Zeitschr. für Wiss. Zool., t. XXIX.

E questo un lavoro assai pregiato nel quale compariscono descritti alcuni nuovi o poco noti lerneopodidi e di essi l'a. fa conoscere con molta precisione ed esattezza la struttura delle parti boccali e le antenne; accompanna la descrizione con buone figure e descrive per talune forme anche il maschio. Le specie descritte sono le seguenti: Achtheres Selachiorum n. sp.; Bracbiella Pastinacne Baird.; Anchorella hostitis Heller.; A. Sargi n. sp.; A. fallax Heller.; A. emarginati Kr.; A. Scombri n. sp.; A. Triglae Claus; Ceslopoda amplectens n. sp. (Naobranclia cygniformis Hesse); Cest. Lizae Kr.

Infine espone i risultati di un confronto fra le estremità di questi ed altri lerneopodidi.

1877. - 2. Eunicicola Clausii, ein neuer Annelidenparasit. Wien.

I801. LAMARCK DE, G. B. P., 1. Système des animaux sans vertébres.

L'a. classifica i lerneidi fra i molluschi.

1809. - 2. Philosophie Zoologique.

L'a. esclude i lerneidi dalla classe dei molluschi e li pone in quella degli anellidi. Piu tardi nell" "Extrait du Cours de Zoologie» 1812 , egli indica la necessita di formare una classe distinta per riceverli, che egli chiama degli Epizoaires, una serie di animali che non puó riferire esattamente a nessuna classe determinata di esseri viventi. 
I8I6. LAMARCK DE, G. B. P. 3. Hist. Nat. Anim. sans vertébr. I." ediz. I816 (altra ediz. I838).

L'a. ponendo le lernee tra gli Epiz̧oaria cosi si esprime: « questi animali s'avvicinano ai vermi e agli insetti, senza somigliare agli uni e agli altri ${ }$.

1792-I805. LATREILLE P. A., J. Histoire naturelle générale et particuliére des crustacés et des insectes, I4 vols. Paris.

Nel vol. dell'anno 1802 presenta notizje sul gen. Caligus dandoci dettagli fatti conoscere precedentemente da Müller e da altri. I caligidi formano qui il $20^{\circ}$ ordine dei Pneumonura.

1825. LATREILLE M., 2. Familles naturelles du règne animal, exposées succinctement et dans un ordre analytique, avec l'indication de leurs genres; I825. Till tyska af Bertholds: Natürliche Familien des Thierreichs etc. 1827 .

18i6. LeACh D. , 1. Articolo «Annulosa»-- Encycl. Brit. Supp. I. r816, p. 405. Sono descritti e figurati Cecrops Lalreillii Leach e Pandarus bicolor Leach.

Quest' aut. introduce il gea. Caligus Müller e descrive una specie britannica Caligus Mülleri Leach ( $=$ C. curtus Müll.).

1819. - 2. Articolo «Entomostraca»-Dictionnaire des Sciences Naturelles. Introduce il gen. Caligus di Müller e descrive questo caligide. Inoltre descrive Pandarus Carchariae Leach.; Nogagus Laireillii Leach.

I 855. LEIDY J., 1. Ueber Cepon distortus sehr Kurz beschrieben in: Contributions tovards a knowledge of the marine invertebrate. Fauna Philadelphia.

I888. - - A Crustacean parasite of the Red. Snapper (Lutjanus Blackfordi) Anchorella fasciculata. - Proc. Acad. Nat. Sc. Philad. 1888, p. I38I 39 .

1888. - 3. Parasitic Crustacea (Lernaeonema procera n. sp.) - Proc. Acad. Nat. Sc. Philad. I888, p. 165.

I889. - 4. A parasitic Copepod (Chalimus tenuis) - Proc. Acad. Nat. Sc. Philad. I 889 , p. 95 .

I85 I. LEYDIG FR., I. Ueber ein neues parasitisches Krustenthier. - Wiegmann's Archiv f. Naturgesch. XVII, I, p. 259-262.

E descritto un nuovo copepode che l'a. chiama Splaterosoma Corzinae e che trovó nei canali mucipari di una corvina.

I853. -- N. Neuer Schmarotzerkrebs auf einem Weichthiere. - Zeitschr. f. Wissensch. Zoolog. IV, p. 377-382, Taf. I4.

L'a. descrive un nuovo copepode parassita della Doris lugubris ossia la Doridicola agilis che sembra a Nordmann (1865) identica al gen. Arfotrogus.

I824. Lesueur C. A., On three new Species of Parasitic Vermes, belonging to the Linnaean Genus Lernaea. - Read February 17, I824. 
I859. LeUCKart R., 1. Ueber die Gesichtswerkzeuge der Copepoden. - Wiegmann's Archiv. f. Naturgesch. XXV, x, p. 247-262, Taf. 6 u. 7.

1859. - 2. Carcinologisches. Ueber Notopterophorus Costa. - Wiegmann's Archiv f. Naturgesch. XXV, x, p. 24I-247, Taf. 6.

1877. Levinsen G. M., Om nogle parasitiske Krebsdyr, der snylte hos Annelider. Kjobenhavn, I877, $8 \mathrm{n}$. Kpfrt.

Sono descritti cop. par. di anellidi: Selioüdes Bolbroei Lev. n. g. e sp. - Rhodinicola elongala Lev. n. g. e sp. - Herpyllobius arclicus Stp. Ltk. (Silenium Polynoës Kr.) - H. crassirustris Sars (Silenium crassirosire Sars) - Bradopbila pigmaea Lev. n. g. n. sp. - Saccopsis Terrebellidis Lev. n. g. n. sp. (Herpyllobius articus? Stp. Ltk.) - Crypsidomus Terebellae Lev. n. g. n. sp.

I 85I-52-55. LiljeboRG W., Om Hafscrustaceer vid Kullaberg i Skane. - Ofvers. af K. Vet. Akad. Förh.

I855. LiNDSTRöM G., Bidrag till Kännedomen om Östersjöns evertebrat-fauna. - K. Vet. Akad, Förh.

I746 e I761. LinNeo C. A., 1. Fauna suecica. - Stockholmiae (1. ${ }^{3}$ ediz. 1746).

Nella prima ediz. del i746 l'a. descrive un animale parassita trovato sopra Cyprinus carassius. Stabilisce da questa specie il gen. Lernaea. L'a. descrive pure brevemente un Pediculus Farionis, evidentemente una specie di Caligus.

Nella seconda ediz. aggiunge una lernea che abitando le branchie del salmone fu detta Lernaea salmonea, stata descritta e figurata da Gisler negli Act. Holmens I75 I col nome di Pediculus salmonis.

1747. - 2. Iter Vestrog, I71.

L'a. dà notizie di una specie di Lernaea trovatà sulle branchie di una specie di Gadus: Lern. asellina.

1758. - 3. Systema Naturae (10. ${ }^{\text {th }}$ ed.), I, Holmiae (Stockholm).

I766. - 4. Systema Naturae (I 2. ${ }^{\text {th }}$ ed.), Stockholmiae (Stockholm) - (I $3{ }^{\text {th }}$ ed.), Vindobonae (Vienna), 1767 .

L'autore ammette l' esistenza di 4 specie di lernee (forme di cop. par. che egli cosi chiama) e che cataloga tra i vermi. Non sembra conoscere i Caligus o li confonde con gli Argulus.

1889. LöNNBERG E., Biđrag till Kännedomen om fritt lefvande Caligider. - Verh. Biol. Ver. Stockholm, Bd. I, p. 148-158.

1887. Lucas H., 1. Sur le Shaerifer cornutus Rich. - Ann. Soc. Entomol. France (6) T. 7, I Trim. Bull., p. LI.

1887. - 2. Sur le Cecrops Latreillii. - Ann. Soc. Entomol. France (6) T. 7, r. Trim. Bull., p. XXXI-XXXII.

1860. LUBвоck J., On some oceanic Entomostraca collected by Capt. Toynbee. Transact. of the Linnean soc. of London, XXIII, p. I73-I92, pl. 29,

L'aut. descrive il Bacalus elongalus Lubbock, che è secondo Mrázek una forma giovanile di Pennella. 
1897. Malaquin A., 1. Evolution des Monstrillides (Haemocera n. g. Danae Clpd. et Haemocera filigranarum n. sp.) - C. R. Acad. Sc., Paris, T. 28, n. 2, p. 99-102. - Extr. Revue Scientif. (4), T. 7, n. 2, p. 54.

I900. - 2. Nouvelles recherches sur l'évolution des Monstrillides. - C. R. Acad. Sc., Paris, T. 30, n. 7, p. 427-430. - Extr. Revue Scientif. (4), T. 13, n. 8, p. 248 .

I 90I. - 3. Le parasitisme évolutif des Monstrillides. - Arch. Zool. Expèr. (3), T. 9, n: I, avec 3 pls., p. $8 \mathrm{I}-\mathrm{I} 60, \mathrm{n} .2$ avec, 4 pls., p. I6I-218, 232; oppure in: Ausz. von F. Zschokke Zool. Centralbl. 9 Ihg. n. 2, p. 53-59 - Abstr. Journ. R. Micr. Soc. London, I902, P. I, p. 46-47.

Igor. - 4. La Thaumatoëssa armoricana Hesse, et les phénomènes de dégénérence pendant la vie libre des Monstrillides. - Bull. Soc. Entom. France, n. I2, p. 216-219.

I863. MaLm A. W., Nya fiskar kräft-och blötdjur Göteborgs. - K. Vet. o Vitterh. Samh. handl. VIII - anche nelle: Zoologiska Observationer,

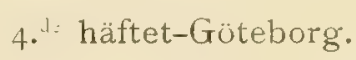

É qui descritto il lerneopodide Vanbenedenia Kröyeri Malm., p. I14, pl. I, che nel mare di Kattegat l'a. ha riscontrato quattro volte, femmine sole, fissate alla spina della natatoria dorsale anteriore di Chimaera monstrosa.

I879. Mayer P., Carcinologische Mittheilungen. - V. Pennella und Conchoderma. VII. Ein neuer parasitischer Copepode. - Mittheil. Zool. Station Neapel, I Bd. p. 53 und 515-521, Taf. I7.

(n. gen. Ine Balanoglossi n. sp.)

Nella prima nota l' a. fa osservare Pennelle (P. filosa L.?) di Xiplsias gladius, che portano Conchoderma (Cirrjpede) fissato alla loro parte posteriore. Lo stesso fatto è segnalato da Giard. Nella seconda nota l'a. descrive il n. gen. di copepode Ine parassita del Balanoglossus.

I824. Mayor., Notice sur une nouvelle espèce de Lernaeopoda. - Bullet. Scienc. Soc. Philomat., Vol. XXIV, p. 24-25.

Sulla Lernaeopoda stellala e salmonea.

1899. Mesnil Fél. et Cauliery M., Evolution des Monstrillides. - Proc. IV. Internat. Congr. Zool. Cambridge, p. 22 \%.

1868. METZGER, Ueber das Männchen und Weibchen der Gattung Lernaea vor der Eintritt der sogenannt rückgeschreitenden Metamorphose. - Nachrichten Gesellsch. Wiss. Univ. Göttingen anche in: Archiv. f. Naturg. Tahrgang XXXIV).

L'a. studia il $\sigma^{\prime}$ e la $\&$ della Lernaea nello stadio larvale che precede la metamorfosi regressiva, cioè a dire, nello stadio dell' accoppiamento. 
1904. Miculicich M., 1. Thynnicola Ziegleri n. g. n. sp. -- Zool. Anzeiger, I 3 Sept. I904, p. 47, Bd. XXVIII, n. 2.

L' autore descrive un copepode parassita del tonno che egli ritiene come nuovo, mentre che invece sarà più tardi (solo pochi mesi dopo) riconosciuto da Stenta per Brachiella Thynni Cuv.

I905. -- 2. Zur Kenntnis der Gattung Brachiella Cuv, und der Organisation der Lernaeopodiden mit. 7 fig. - Zool. Anzeiger Bd. XXVIII nr. 18 Leipzig.

Miroslaw Miculicich (1905) con questo pregiato lavoro (pubblicato estes. nel Zeit. für Wiss. Zoolog. 1905) porta un contributo notevole sulla conoscenza anatomica e istologica del gen. Brachiella finora poco noto sotto questo aspetto. Avendo ottenuto buoni preparati di sezioni egli é stato in grado di porgerci chiara rappresentazione dell' organizzazione interna di questi copepodi parassiti, di cui studia pure la struttura istologica.

I 905. - 3. Weitere Mitteilungen zur Kenntnis der Gattung Brachiella Cuv. Zool. Anzeiger Bd. XXVIII. n. 21/22, II April 1905.

1877. Mrers. Lernaeopoda arcturi. -- Ann. Mag. N. H. (L.) xx, 1877.p. 106, oppure in: Nares' "Narrative of a Vogage to the Polar Sea " II Crustacea, p. 247 .

1833. Milne-EDwards, H., 1. Mèmoire sur l'organisation de la bouche chez les Crustacès suceurs. - Ann. đ. Scienc. Natur. Vol. XXIX. p. 78-86.

L'a. studia la struttura e la formazione delle parti boccali nei cop. parassiti (Siphonosthomi)

1840. - 2. Histoire naturelle des crustacès, comprenant l'anatomie, la physiologie et la classification de ces animaux. Paris.

E un importantissimo lavoro in cui sono passati in rassegna e descritti tutti i crostacei, compresi anche $i$ copepodi parassiti, conosciuti fino al 1840 , distribuiti in ordine sistematico e divisi in generi, faniglie e ordini.

L' autore ordina i cop. par. in un prospetto sistematico, fondato su caratteri artificiali, ma che ha il merito d' essere stato il primo tentativo di classificazione di qualche valore.

1786. MODER, Acta suecica p. 256 u. ff.

Intorno alla Pennatula sagitta e filosa.

1895. MrAzek AL., Uber Baculus Lubb, und Hessella Br. Ein Beitrag zur Ana tomie der Lernaeiden. Mit 2 Taf. und 2 Holzchn. - Sitzgsber. K. Böhm. Ges. Wiss, Math Nat. Cl. i895. XLIV. Ausz. vom. Verf. Zool. Centralbl. 3 Ihg. n. 7. p. $237-238$.

L'a. riconosce nel Baculus elongatus di Lubbock una forma giovanile di Pennella, così pure nell' Hessella cylindrica Brady, nota la stessa cosa.

1852. MÚlLER FR., Eine Beobachtung über die Beziehung der gattungen Caligus und Chalimus - Wiegmann's Archiv. f. Naturgesch. XVIII, I. p. 9 I-92.

L'a. rileva che i Chalimus non sono che forme larvali di certi caligidi. 
3777. Müller Otho Fr., 1. Zoologia Danica.

L'a. introduce il genere Caligus sotto il nome di «Binoculus" adottando questo nome da Geoffroy, ma pjù tardi nel 1785 sul suo Entomostraca fonderà il gen. Caligus.

I785. - 2. Entomostraca, seu Insecta testacea quae in aquis Daniae et Norvegiae reperit, descripsit, et iconibus illustravit. Lipsiae et Hafniae. (Leipzig and Copenhagen).

L'a. fonda il gen. Caligus.

i869. Munter D. Jul. u. BuchHolz D'., Beitrag zur carcinologischen Fauna Deutschlands. - Mittheil. aus dem naturwissensch. Vereine von Neu-Vorpommern und Rügen. Berlin.

Il gruppo dei parassiti (Siphonostoma Latr.) è rappresentato da: Argulus foliaceus L. - Acbtheres Percarum Nordn. Lernaeocera cyprinacea sul Cothus gobio - Lernaeopoda carpionis Kröy. - Ergusilus gibbus Nordm. - Ergasilus Sieboldii Nordm. branchie del Cyprinus Jeses - Lernaea branchialis L. - Basarisiles salmoneus M. Edw. - Tracheliasles maculatus Kollar - Tr. polycolpus Nordm. branchie di Cypr. Blicca.

1832. NordmanN A. v., 1. Mikrographische Beiträge zur Naturgeschichte der wirbellosen Thiere, II Heft. Berlin.

É importantissima la comparsa di questa pregiata memoria nella storia dei copepodi parassiti, perché è da q̨uesto momento che detti animali assunsero il fosto naturale nella classe dei erostacei. L'a. studid non solo una grande quantità di forme nuove facendo osservazioni sulla loro struttura esterna, ma anche rileva i caratteri salienti dell' organizzazione interna e ne segue lo sviluppo larvale.

Tralascio di citare le specie studiate in questa memoria, perchè è opera fondamentale che non può essere sconosciuta, anzi dev' essere consultata sovente, da chiunque studia copepodi parassiti. Ricorderò soltanto che più importante di tutto è la seconda parte (II. zweite abhandlung. Beitrag zur Naturgeschichte der Lernaên) ove è trattato magistralmente di una forma d'acqua dolce l'Achbheres Percarum, di cui lo studio sarà più tardi ripreso e completato da Claus, ed è stato il fondamento per le ricerche ulteriori intraprese da questo autore e da altri sui lerneidi.

I864. - 2. Neue Beitraege zur Kenntniss Parasitischer Copepoden. Erster Beitrag. - Bullet. d. 1. Soc. des Natur. de Moscou, XXXVM, 2. p. 46I-520, Taf. 5-8.

In questo secondo importante lavoro l'a. aggiunge altre notizie sui copepodi parassiti e descrive altre n. forme sia di generi sia di specie. Fra i generi nuovi egli descrive Strabax, Pseudulus, Norion, Donusa, Stalagmus, Peniculus. Oltre a ció descrive specie non conosciute ancora dei gen. Pernella, Tucca, Lemantbropus.

L'a. premette alla memoria un elenco bibliografico delle opere più utili riguardanti i cop. par. studiati fino a suoi tempi e riporta un prospetto sistematico delle famiglie e dei generi secondo Steenstrup e Lütken (IS6I).

1863. Nrström C. L., Iakttagelser rörande Faunan è Jemtlands vattendrag. Akademisk Athandling. I863.

1815. OkeN L., Lehrbuch der Naturgeschichte. Th. III. p. I84. ff. und. p. 357. ff. In questo manuale di storia naturale l'autore segue Linneo e pone le lernee fra i molluschi, è però il primo a cominciare a dividerle in diversi gruppi generici. 
I868. Olsson P., 1. Prodromus faunae Copepodorum parasitantium Scandinaviae Lunds Univ. Arsskrift. Tom. V.

É un lavoro di valore faunistico e sistematico. L'a. enumera e descrive con diagnosi latina una moltitudine di cop. par. propri della fauna scandinava, distribuiti in ordine sistematico. La ricerca delle specie è facilitata con tabelle sinottiche.

Le n. sp. descritte sono le seguenti : Nogagus socialis; Echthrogaleus perspicax; Chondracanthus annulatus; Lernaeopoda Edwardsï; L. Tongimana n. subsp.

La memoria è accompagnata da 2 tavole.

1870. - 2. Nova gen. parasit. Copepod. et Platyhelminth. Lund.

Fra i cop. par. l' autore presenta l'Enalcyonium rubicundum n. sp. in Alcyonio digitato, che egli descrive in latino; e il Lamippe rubra, parass. della Pennatulu rubra, descritto da Bruzelius.

1872. - 3. Om ein ny parasitisk Copepod. (Lernaeopoda clavigera) med. I taf. - Ofversigt af Kongl. Vetenskaps-Akademiens Förhandlingar, 29. Arg. I 872 . (1873) n. 5 p. $63-65$.

1877. - 4. Om parasitiska Copepoder i Jemtland. - Ofversigt af Kong1. Vetenskaps-Akademiens Förhandlingar., n. 5, I877. Stockholm.

L'a. cita e descrive accompagnando con diagnosi latina le descrizioni, e con figure, alcune specie nuove: Caligus borealis n. sp: Lernaeopoda Maraenae n. sp.: L. alpina n. sp. : L. Lotae n. sp.

1896. - 5. Sur Chimaera monstrosa et ses parasites: - Extrait Mèmoires de la Soc. Zool. de France. Tom. IX, p. 499, anneè 1896.

Fra i par. di altre classi di animali, per questo pesce, l'au. cita i seguenti copepodi: Caligus curtus Müller; C. rapax Miln. Edw.; Vanbenedenia Kröyeri Malm.

I86I. Pagenstecher A. H. D-, Thersites gasterostei, eine neue gattung parasitischer Crustaceen. - Wiegmann's Archiv. f. Naturgesch. XXVII. I. p. II 8 - I26 Taf. 6 .

L'a. descrive come nuova questa specie Thersites gasterostei Pagen. (sinonima di Ergasilus gasterostei $\mathrm{Kr}$.).

? Pallas. Elenchus zoophyt. p. 364. Sopra la Pennatula (Pennella).

PARAsitic Crustacean on a Flying-Fish (Pennella Blainvillii) With fig. Bull. Liverpool Mus. Vol. I. n. I. p. 23-24.

É descritta la $P$. Blainvillii parassita di un pesce volante.

I893. Pedaschenko D. D., 1. Sur 1a segmentation de l' œuf et la formation des feuillets embryonnaires chez la Lernaea branchialis L. Note prèliminaire. - Revue Sc. N. Petersbourg, $4^{e}$ anné, p. 197-199. II figg.

1897. - 2. Uber die Entwicklung des Nervensystems und der genitalzellen und die Dorsal organe von Lernaea branchialis. - Arb. Nat. Ges. Petersbourg. Tome 37, I I pagg. oppure: Lernaea branchialis in: Trav. Soc. Imp. Natural. S. Petersbourg. Vol. 27. Livr. I. C. R. 1896. n. 6. p. 187-194. 
1899. Pedaschenko D. D., 3. Lernaea branchialis L. Die Embryonalentwicklung und Metamorphose von Lern. branch. Mit 6 lith. Doppeltaf. u. einem deutsch. Rèsumè. - Trav. Soc. Imp. Natural. St. Petersbourg. Vol. 24. Livr. 4. p. 1 - 229, 230, 246. Rèsumè p. 247, 295, 296, 310 (III p.)

In questi lavori di Pedaschenko è studiata a fondo l'embriogenesi della Lernaea branchialis.

1770. Pernetty A. J., Voyage aux Iles Malouines. Paris.

L'a. descrive senza nominarlo, un anim. parass. riconosciuto oggi per Brachiella Thynni ne dà i disegni (p: 93, T. I. P1. I figs. 5, 6).

I838. Pickering Ch., M. D. and. DANa J. D., Description of a specie of Caligus (C. Americanus). - Silliman's Americ. Journ. of Science, XXXIV, p. 225-266, pl. 3-5. Read before the Yale Nat. Hist. Soc. Feb. 20, 1838.

L'a. descrive il Caligus americanus ne' suoi caratteri esterni come in quelli interni, e accompagna questo studio con splendide figure. Ė opera di molto pregio per quei tempi.

1902. Poche Fr., Bemerkungen zu der Arbeit des Herrn Bassett - Smith : \&A Systematic Description of Parasitic Copepoda found on Fiches, with an Enumeration of the Known Species » Zool. Anz. 26 Bd. n. 685, p. 8-20.

L' a. fa osservazioni eritiche a riguardo di un lavoro di Bassett-Smith, indicato dallo stesso titolo.

Giustamente propone la sostituzione del nome n. Orthagoriscicola per il gen. Laemargus copepode parassita, il cui nome era stato occupato prima tra i pesci da Müller e Henle : cosi pure propone per il gen. Lophoura Kollar, quello di Rebelula n. nuovo, come anche quello di Hatschekia per il gen. Clavella di Oken, mentre stabilisce che con questo ultimo nome venga distinto il genere Anchorella. Tutto cio in merito alle leggi della Nomenclatura zoologica approvate nel i8gs.

1906. QuIDOR A., Sur le Leposphilus labrei Hesse et sur la famille des Philichthydae. Comunicazione nella seduta del 22 Gennaio Igo6 all'Acadèmie des Sciences. (Cosmos, 55. annèe N. S. n. 1097 p. I35, 3 fèvrier 1906).

1885. Raffaele F. e Monticeli F. S., Descrizione di un nuovo Lichomolgus parassita del Mytilus gallo-provincialis L. - Mem. R. Accad. Linc. (4), Vol. I.०

1884. Rathbun Rich., 1. Annotated List of the described Species of parasitic Copepode (Siphonostoma) from American Waters contained in the United States National Museum. - Proceedings U. S. Nat. Mus. Vol. 7, p. 483-492.

L' autore dà un elenco delle specie di copep. parassiti dei mari americani contenuti nel Museo Nazionale degli Stati Uniti.

1887. - 2. Descriptions of parasitic Copepoda belonging to the genera Pandarus and Chondracanthus. With 7 pl. - Proc. U. S. Nat. Mus. Vol. 9, p. 310-324 - Abstr. in: Journ. R. Microsc. Soc. London, 1887, P. 3, p. 395 (4 n. sp.).

L'a. descrive quattro nuove specie appartenenti ai generi Pandarus e Chondracanthus. 
I887. Rathbun Rich., 3. Descriptions of (4) new. species of parasitic Copepods belonging to the genera Trebius, Perissopus and Lernanthropus. Proc. U. S. Nat. Mus. Vol. Io, p. 559-57I.

L'a. descrive le seguenti nuove specie: Trebius temuifurcatus; Perissopus communis colla varietà Stimpsoni; Lernanthropus Brevoortiae; L. Pomatomi, e ne dà le relațive figure distribuite in 7 tavole.

1836. RAthke H., 1. Bemerkungen uber den Bau der Dichelesthium Sturionis und der Lernaeopoda stellata mit. I pl. Nov. - Act. Acad. Leopold. Carol. Vol. XIX, p. I25-I68.

Studio anatomico del Dichelesthium, da esemplari conservati in alcool.

1837. - 2. Ueber Lernaeopoda stellata zur Morphologie. - Reisebemerkungen aus Taurien, p. 35 .

1843. - 3. Beitrag. zur Fauna Norwegens. - Nov. Act. Acad. Leopold. Carol. Vol. XX, I. p. I-264. mit. I 2 Taf. Bonn.

L'a. tratta del Caligus curtus, C. diaphanus e hippoglossi, Nicothoe astaci, Chondracanthus Lophii, Ch. gibbosus 2 e + , Lernaea branchialis e Peltogaster paguri et carcini (Cirripedia).

1829. Retzius A., Beskrifning öfver en ny Skandinavisk Lernaea fran Nordsjön, Kallad Lernaea Dalmanni. - Kong1. Vetensk. Akad. Handling. Stockholm p. Iog-II9, c. tab. oppure: Beschreibung einer neuen Scandinavischen Lernaea aus dem Nordsee, Lernaea Dalmanni gennant. Froriep Notizen Bd. XXIX. p. 6. fig. 5-9, I8zo. Idem in Isis. p. I 345 , Taf. IX, I83I.

L'a. descrive la Lernaea Dalmanni (Charopinus Dalmanni)

I900. RICHARD J., Essai sur les parasites et les commensaux des Crustacés Arch. de Parasitol. T. 2. n. 4 p. 548-590, 59I-595.

L'a. per quanto riguarda i copepodi, parla delle scoperte di Hansen sulla famiglia dei Coniostomatidae parassiti e commensali di altri crostacei e dà la lista di tutte le forme nuove di questa famiglia fatte conoscere da quel naturalista,

1870. RICHIARDI S., I. Intorno ad una n. sp. del gen. Bomolochus (B. Ostracionis) con fig. - Arch. per la Zool., l'Anat. e 1a Fisiol,, 2 Ser., Vol. 2, fasc. I, p. $47-59$.

1876. - 2. Sopra lo Sphaerifer cornutus Rich., Sphaerosoma Corvinae Leydig ed una nuova specie del gen. Philichthys Steenstr., Ph. Sciaenae Rich. con I tav. - Atti Soc. Tosc. Sc. Nat. Pisa, Vol. 2, fasc. 2, p. 99-III.

1876. - 3. Intorno al Peroderma cylindricum dell' Heller e sopra 2 nuove specie del gen. Philichthys. - Atti Soc. Tosc. Sc. Nat., Pisa., Vol. II, fasc. $2^{\circ}$ ed ultimo.

L'a. oltre ad uno studio sul gen. Peroderma ed alla specie P. cylizdricum presenta la descrizione del Philichthys Edwardsii e del Plilichthys Steenstrupii, e di essi dà le figure. 
1877. RICHIARdi S., 4. Descrizione di due specie nuove di Lernaeenicus (L. neglectus, L. vorax) con osserv. intorno a questo ed ai gen. Lenaeocera B1. e Lernaeonema M. Edw. - Atti Soc. Tosc. Sc. Nat. Pisa, Vol. III, fasc. 1 .

1877. - 5. Descrizione di cinque specie nuove del gen. Philichthys ecc. - Atti Soc. Tosc. Sc. Nat. Pisa, Vol. III, fasc. I, p. 166.

L'a. descrive il Philichthys Lichiae, Ph. Denticis, Ph. Pagri, Ph. Pagelli, Ph. Baraldii e una nuova sp. del gen. Sphaerifer, Sph. Leydigii, e dà di tutte queste n. sp. le figure.

1877. - 6. Dei Filictidi : osservazioni critiche e descrizione di 6 n. sp. - Atti Soc. Tosc. Sc. Nat. Pisa, Vol. III, fasc. I, p. 180.

L'a. descrive e dà le figure dei seguenti: Philichllays Sieboldii; $P$. minimus: $P$ h. Grubeit; Ph. Agassizii; Ph. Haeckelii; Ph. Muraenae.

Propone il termine nuovo (Polyrrbyncluss) da usarsi per distinguere questo genere Philichtys e parla del gen. n. Colobomatus di Hesse che, occorre, sia meglio studiato e descritto per ammetterlo e fissarne la sua posizione nella famiglia dei filictidi. Dichiara che il gen. Splsaerifer fa parte di questi e in ultimo presenta una lista completa di 20 Filictidi noti fino al 1876 .

1878. - 6a. Tripaphylus musteli. - Processi verbali Soc. Tosc. di Sc. Nat. in Pisa, p. XX.

1879. - 7. Sopra 5 sp. nuove di crostacei parassiti. - Processi verbali, Soc. Tosc. Sc. Nat. Pisa, Marzo 1879, p. LXXXI.

(Lernanthropus brevis Rich.; L. vorax Rich. ecc.).

1880. - 8. Sopra due nuove specie di crostacei parassiti. - Proc. verb. Soc. Tosc. Sc. Nat. Pisa, p. XXVI; Zool. Anz., n. 48, p. 69, 3 Ihg.

Brachiella ramosa del pesce spada; Philichlhys fatolae dello Stromateus fiatola Linn.

I880. - 9. Catalogo sistematico dei crostacei che vivono sul corpo degli animali aquatici - Catalogo Sez. Ital. Esposiz. Internaz. di Pesca. Berlino, I880 - Firenze - anche in Pisa tip. Vannucchi 1880 , (8 p.).

L'a. dà un catalogo generale delle specio di cop. par. viventi nel Mejiterraneo, raccolte da lui stesso, e di cui molte sono nuove, parte da lui stesso descritte in altre note, parte ancora da descriversi.

I88I. - 10. Intorno a due specie nuove di crostacei parassiti. - Zool. Anz. 4. Iahrg. n. 88 , p. 386-387.

L'a. descrive il Peroderma Petersi che vive nel corpo del Gobius buccatus Cuv. Val. e il Chondracanthus Bleckeri che vive aderente alle laminette branchiali del Cheilinus cỉlorurus Bl. e del Pseudorbombus Russeli Gray.

I88๘. - 11. Sopra due nuove specie di crostacei parassiti. - Zool. Anz. 4 Iahrg, n. 92, p. 504-505 - anche Processi verbali Soc. Tosc. Sc. Nat. Adunanza 5 Luglio I88 I.

L'a. descrive Tracheliastes gigas e Lernanthropus Polynemi vivente sul Polynemus tetradactylus Shaw. 
I882. RICHIARDi S., I2. Intorno ad una nuova specie del genere Peroderma.' Zool. Anz. n. 120, Vol. V, p. 475-476 - Processi verbali Soc. Tosc. Sc. Nat. Adunanza 7 Maggio 1882.

Colla presente nota 1'a. fa conoscere la rara specie Peroderma Bellottii, che vive sullo Scopelus Benoiti.

1882. - 12a., Descrizione di una specie nuova del gen. Chondracanthus - Processi verbali Soc. Tosc. Sc. Nat. Pisa, Vol. III, p. I54, anche: Zool. Anz. 5. Iahrg. n. I21, p. 504 .

1883. -- 13. Descrizione di una sp. n. di crost. par. Philichthys Döderleini. Zool. Anz. 6 Iahrg, p. 558-59, oppure: Atti Soc. Tosc. Sc. Nat. Pisa, Vol. III, p. 279-280.

Detto copepode è parassita del Labrus lurdus.

1885. - 14. Descrizione di due specie nuove del gen. Lernanthropus. - Proc. verb. Soc. Tosc. Sc. Nat. Pisa, Vol. IV, p. 82-84.

L'a. descrive Lernanthropus Micropterygis su Micropteryx dumerili Risso, p. 82 (Palermo) e Lern. Tylosurus su Tylosurus imperialis Raf, , p. 83.

1876. Richters F., Caligus lacustris Stp. u. Ltk. o7. - Verh. d. Ver. f. Naturwiss. Unterhalt. Hamburg. 2 Bd., p. 244-245.

L' aut. descrive il Caligus lacustris ơ.

18I6. Risso A., 1. Histoire naturelle des Crustacés des Environs de Nice. - Paris.

L'a. fra i crostacei d'ordine inferiore non cita che due copepodi parassiti: Caligus productus Müll. e C. imbricatus Risso.

I826. - 2. Hist. nat. des principales productions de l'Europe meridionale, 5 Vol. Paris.

L' autore nella parte che riguarda i crostacei cita parecchi copepodi parassiti: Caligus minimus Otto; Otrophesa inbricata Risso (Anthosoma crassum Abildgaard); Nemesis Lamnae Roux; Chondracanthus Lophius Risso; ? Pandarus Rouxi Risso.

1888. Rosolt D.r A., Ueber zwei neue an Echinodermen lebende parasitische Copepoden.

L'a. descrive Ascomyzon Comatulae und Astericola Clausii viventi sugli echinodermi.

1828. Roux P., Crustacés de la Mediterranée. - Paris.

Quest' autore dà le figure di molte specie di macruri e brachiuri ed anche di isopodi del Mediterraneo; non trascura tuttavia di descrivere una forma di cop. parass. Nentesis Lamnae di cui dà una buona tavola (tav。 XX, fig. I-9).

1868. Salensky, Sphaeronella Leuckarti, ein neuer Schmarotzerkrebs. - Archiv. fur Naturg. Vol. XXXIV.

L'a. descrive un nuovo copepode parassita di un Amphithoe (?).

I86r. SARs M., 1. Beskrivelse med Afbildninger af fire nye parasitiske Copepoder. - Christiania's Vidensk. Selskab. Forhandl. for 186I, p. I34-I4I.

L'a. descrive in questo lavoro $4 \mathrm{sp}$. nuove: Eolidicola tenax, Terebellicola reptans, Sabelliphilus elongalus, Chonephilus dispar: i tre ultimi parassiti di anellidi. 
r861. SaRs M., 2. Beretnnig om et nyt lernaealignende Krebsdyr, Sabellacheres gracilis Sars. - Christiania's Vidensk. Selskab. Forhandl. for 186 I p. I 4 I-I 43 .

L'autore rifesce intorno al Sabellacheres gracilis Sars copepode par. il cui posto sistematico è ancora dubbio, sebbene Gerstaecker con poca certezza e provvisoriamente lo comprenda tra i Dichelesthiina.

1870. - 3. Bidrag til Kundskab om Christianiafjordens Fauna. II. Crustacea etc. Nyt Magazin for Naturvidenskaberne Bd. I7. Christiania I870.

Sono descritti: Melinnacheres ergasiloudes Sars; Herpyllobius crassirostris; Eurysilenium truncatum, parassiti di anellidi.

1876. Schatr R., Uber Chondracanthus angustatus Heller - Aus dem Bande der Sitzb. der K. Akad. der Wissensch. I. Abth. October-Heft. Jahrg 1876.

L'a. studia la detta specie, di cui ha trorato parecchi esemplari presso Trieste, nella sua esterna e interna struttura e anche nella forma maschile; la memoria è illustrata da 3 buone tavole.

1896. Schimkewtsch WL. M., 1. Studien über parasitische Copepoden, Mit 3 Taf. u. I fig. im Text. - Zeitschr. f. Wiss. Zool. 6r. Bd., 3. Hft., p. $339-35^{8-362}$.

(Chondracanthus gibbus Kr.; Ch. Merlucii Holt.; Notopterophorus (Doropysus) gibbus Thor. und N. papilio Hesse).

1896. - 2. Sur les premiers stades du développement des Copépodes parasitaires. C. R. 3. Congr. Internat. Zool. Leyde. p. 503-504.

L'a. studia l'embriogenesi specialnente di Chondracanthus, Notopterophorus, Tracheliastes, Enteropsis.

I898. - 3. Zu einem Referat des Herrn Prof. D.r R. S. Bergh.-- Zool. Anz. 2 I Bd. N. $55^{\circ}$, p. 48 (Uber Entwicklung von Lernaea).

1899. - 4. Einige Worte über die Entwiclung der parasitischen Copepoden. - Zool. Anz. 22 Bd. N. 58I, p. III-II4.

Igor. Scotr A., 1. Some Additions to the Fauna of Liverpool Bay. - From Trans. Liverpool Biol. Soc., Vol. XV.

In aggiunta alla fauna nota di Liverpool l' autore oltre a vari altri nomi di animali, aggiunge quelli di 12 specie di cop. par. fra cui i nuovi generi e n. sp. seguenti. Egli li descrive e ne dà le figure: Caligus brevicaudatus n. sp.; Pseudocaligus n. g., Ps. brevipedes (Bassett-Smith).

Igor. - 2. Additions to the British Fauna (Fish Parasites). With 3 pls. Proc. Trans. Liverpool Biol. Soc., Vol. XV, p. 342-353-- Abstr. Journ. R. Micr. Soc. London I902, P. 2, p. I75.

Sotto diverso titolo è presentato lo stesso lavoro precedente. Vì è descritto il a. g. di copepode Psendocaligus. 
I9or. Scott A., 3. On the Fish Parasites, Lepeophtheirus and Lernaea. With 5 pls. - Rep. for I goo. Lancash. Fish. Labor. Herdman, p. 63-I I0-1 I5, oppure: Abstr. Journ. R. Micr. Soc. London I902. P. 2, p. I85.

Dopo un cenno storico sui cop. par. l'autore tratta del Lepeophtheirus pectoralis di cui ha avuto nelle mani una grande quantita (32) di esemplari, e ne studia i caratteri esterni.

Esamina il contenuto e la forma del loro canale digestivo, studia il sangue e la circolazione, il sistema muscolare, nervoso, gli organi riproduttori e la storia del loro sviluppo.

Passa quindi a parlare dei lerneidi e descrive la Lernaea branchialis Lin. facendo gli stessi studî che per il Lepeophtheirus. Aggiunge molte figure distribuite in 5 tav.

1895. Scotт Th., 1. Entomostr. G. of Guinea. - Trans. Linn. Soc., Zool. VI, I895, p. I 30, pl. XIV, fig. I 9.

Si rileva che il Caligus bengoensis Scott es sin. del Caligus coryphaenae Stp. e Ltk. con cui sono eziandio sinonini C. Thynni? Dana (Expl. Exp. U. S. Crust. II, 1854) e C. sculatus? M. E. (Hist. Nat. Crust. 1840 , p. 453).

Si trovano descritti in questo lavoro le n. sp. Caligus murrayanus e $C$. dubius.

1897. - 2. Asterocheres violaceus (Claus) from the Firth of Clyde. - Ann. of Scott. Nat. Hist., I897, Apr., p. I 27.

1897. - 3. Sabelliphilus Sarsi, Claparède, from the Firth of Forth. - The Scottish Naturalist, I897, Oct., p. 256. Inoltre: Sabell. Sarsi Clap. in the Clyde. - Ann. of Scott. Nat. Hist. 1897, July, p. 156.

I 900 ? - 4. Notes on some Crustacean parasites on fisches. - I 8 Rep. Fish. Board Scottland Pt. 3, p. 144-188, T. 5-8.

E un lavoro di importanza faunistica. L'a. cita 5 I cop., 5 isop., 2 amphip. Fra i cop. par. ricorderd i generi: Thersites, Caligus, Lepeophtheirus, Trebius, Dinematura, Echtrogalens, Pandarus, Laemargus, Clavella, Lernaeenicus, Lernaea, Haemobaphes, Oralien, Chondracanthus, Thysanote, Charopinus, Lernaeopoda, Brachiella e Anchorella. Le 5 nn. spp. appartengono ai gen. Lernaca, Haemobaphes, Chondracanthus, Charopimus, Lernaeopoda.

I90I? - 5. Notes on some parasites of Fishes. - I9 Rep. Fish. Board Scotland, p. I20-I 53, T. 7,8 .

Questo lavoro ha importanza faunistica. Sono ricordate 28 specie di copepodi, dei generi Ergasilus, Caligus, Clavella, Lernaea, Sphyrion, Chondracanthus, Charopimus, Brachiella, Anchorella, Lernaeopoda.

I902? - 6. Notes on some parasites of Fishes. - 20Rep. Fish. Board Scotland, p. 288-303, T. 12, 13.

Sono altre note in cui sono presentate specie di cop. par. della Scozia appartenenti ai generi: Bomolochus, Caligus, Pseudocaligus, Lepeophtheirus, Echtrogaleus, Clavella, Eudaciylina, Haemobaphes, Chondracanthus, Thysanote.

1900. - 7. On Copepods living in the nostrils of Fishes. The Scott. Naturalist, 1900 July, p. I53-155.

Igor. - 8. Clavella labracis Van Ben., a Copepod new to Britain. - Ann. Scott. Nat. Hist., 1901 Apr., p. I20-I I .

L'autore descrive un copepode parassita disegnato da Van Beneden. e che egli riporta come nuovo per la fauna britannica. Oggi il gen. Clavella va chiamato Hatschekia: quindi H. labracis in merito alle leggi della Nomenclatura 1895. 
I 904. Scott Th., 9. Notes on some Rare and Interesting Marine Crustacea. 22 Rep. Fish. Board Scotland Pt. 3, p. 242-261, 3 pls.

(7 nn. spp. in: Monstrilla, Thaumaleus 2, ecc.).

1897. Scott TH. and A.; Notes on Sunaristes paguri Hesse, and some other rare Crustacea. - Ann. Mag. Nat. Hist. (6) XX.

Gli autori descrivono il Sunaristes Paguri Hesse, copepode commensale di Eupagurus Bernisardus (L.).

- Siebold's v., Artikel Parasiten in Handwörterbuch der Physiologie II, p. 66r. Anmerkung 7.

L'a. parla di un crostaceo simile all' Ergasilus, raccolto esternamente sul ventre di Sabella ventilabrum, inoltre di un parassita nella cavità branchiale di Phallusia inteslinalis.

I883. SмIth S. J., 1. List of the crustacea dredged on the coast of Labrador by the expedition of W. A. Searns, in 1882. - Proc. U. St. Nation. Mus. Vol. 6, p. 218-222.

- 2. Review of the marine crustacea of Labrador; ibid., p. 223-232.

L'a. cita per il Labrador la Lernaea branchialis.

1899. Stebbing Thom. R. R., 1. Genus Sphyrion Cuvier.-- Rep. Marin. Biolog. Cape, r898, p. 897 .

L'a, presenta il gen. Sphyrion di Cuvier (sinon. di Lesteira).

- 2. South African Crustacea, - Mar. Invert. South Africa Cape Town. T. 1-4, p. $14-66$.

L'a. tra gli altri crostacei descrive una sp. di Sphyrion.

1862. Steenstrup J, D.r, 1. Philichthys Xiphiae, en ny snylter hos Svaerd-fisken. Overs. Kongl. Danske Vidensk. Selsk. Forhand1. I86 I, p. 295-305, pl. 2. Studio morfologico del- Pbilichtys Xiphiae.

1869. - 2. Om Lesteira, Silenium og Pegesimallus tre af Prof. D. ${ }^{\mathrm{r}}$ H. Kröyer opstillede Slaegter af Snyltekrebs.

E uno studio critico sui tre generi di cop. par, fondati da Krōyer: Lesteira, Silenium e Pegesinallus. L'a. dice il gen. Lesteira sinonimo del gen. Sphyrion Cuvier, la specie Silenium Polinoes Kröyer (1863) sinonima dell' Herpyllobius arcticus Stp. Ltk. (186I), e infine fa notare che il gen. Pegesimallus non fa parte della classe dei crostacei ma si riferisce ad un sifonoforo (Agalmide o Physophora).

i86I. Steenstrup J. and Lütken C., 1. Bidrag till Kundskab om det aabne Havs Snyltekrebsog Lernaeer, etc. - Kongelige Danske Videnskabernes Selskabs Skrifter, $5^{\text {te }}$ Raekke, naturhistorisk og mathematisk Afdeling, V. Kjöbenhavn.

È uno dei lavori più importanti sulla sistematica e sullo studio dei copepodi parassiti dei pesci che si conosca, con 15 splendide tavole e 92 pagine. I generi e le specie sono accompagnate in maggior parte da diagnosi latine e non mancano prospetti per i siugoli generi in cui sono dati i caratteri principali delle specie. Sono descritte moltissime nuove forme. 
1863. Steenstrup J., 2. Det aabne Havs Snyltekrebs etc. (Copepoda parasit. et Lernaea oceani Atl. et Art.) - Arch. Sc. Phys. et Nat. Genève. Nouv. périod. T. I2. I86I, p. I90-I92. T. I6, p. 235.

Gli autori enumerano copepodi parassiti propri dell' oceano Atlantico e Artico.

1852. STEtN F., Ueber die Beziehung der Gattungen Caligus und Chalimus. Wiegmanns Archiv. I852, Heft. I, p. 9I.

1904. Stenta M., Thynnicola Ziegleri Miculicich = Brachiella Thynni Cuv. Zool. Anz., 28 Bd. n. r $\%$, pag. 345 .

L'autore rileva la sinonimia del Thinnicola Ziegleri di Miculicich colla Brachiella Thyıni di Cuvier.

1897. Steuter AdF., 1. Zur Anatomie und Physiologie des Corycaeiden-auges. Zool., An\%. 20. Bd., n. 535, p. $229-232$.

1902. - 2. Mytilicola intestinalis n. gen., n. sp. Aus dem Darme von Mytilus galloprovincialis Lam. Mit 2 fig., - Zool. Anz., 25. Bd., n. 680, p. $635-637$.

L'a. descrive questo n. cop. par. trovato assai frequente nel golfo di Trieste. Il c è grosso circa $4 \mathrm{~mm}$. la of circa $8 \mathrm{~mm}$. Ha riscontrato. in questo crostaceo un sistema sanguigno somigliante a quello che gia Heider ebbe a scoprire nei Lernanthropus.

1882. Stossich M., Prospetto della fauna del mare Adriatico - Bollett. Soc. Adriatica di Sc, Nat. in Trieste, Vol. VII, fasc. I.

Sono citati i copepodi parassiti viventi nell'Adriatico e già notati da Valle.

1762. Stroem, Beschreibung des Bezirks Sandmoer, p. I67.

Sopra la Lernaed adunca.

1847-48. Stroem, (Caligus Strömii Ström.; Laxe lusis Ström. = Lepeoptheirus Strömii Baird.) - Selskabs. Skrifter, Vol. X, p. 23, t. VII, f. 1-7. Kjöbenh.; oppure: Ann. Nat. Hist., 1848, Mai, n. 5, p. $397-$ anche: Trans. Berwick. Nat. Club. 1847.

L'a. rileva l'identità o la sinonimia di Lepeophtheirus Strömiz Baird col Laxe lusis Ström e descrive il Caligus Strömii vivente sulla pelle di Salmo salar L.

187x. SUMPF K., Ueber eine neue Bomolochiden-Gattung nebst Bemerkungen über die Mundwerkzeuge der sogenannten Poecilostomen. Hildesheim.

L'autore nella prima parte descrive la femmina di Taeniacanthus Carchariae n. g. di bomolochide; e nella seconda egli tratta dei Pacilostomi copepodi mancanti di mandibole, e particolarmente descrive l'apparato boccale di Corycaeus germanus of e di Eroasilus Esocis o.

? Thompson? Crustaceen der Britischen Fauna - Archiv. für Naturgeschichte XV Iahrgang, Heft VI, p. 318-339.

L'aut. tratta fra altri crostacei del Caligus Strömii Baird e della Pcnnella pustulosa. 
I843. Thompson W., 1. Report on Fauna of Ireland, div. Invertebrata. - Report of Brit. Assoc. for Advanc. Sciences.

L'a. tratta della Lernaeonema monillaris ecc.

1847. - 2. (Lepeophtheirus Strömi ecc. ecc.) Ann. and Mag. Nat. Hist., Ser. I. Vol. XX.

L'a. tratta dei copepodi seguenti: Lepeophtheirus Strömii; L. pecloralis; L. Nordmanni; Trebius caudatus ecc.

1885. Thompson G., M., 1. I) Parasitic crustacea in: N. Zealand Journ. Sc., Vol. 2, p. 455; 2) New crustacea ibid., p. 576.

1889. - 2. Parasitic Copepoda of New Zealand, with Descriptions of New Species. With 5 pls. - Trans. N. Zealand Inst. Vol. 22 (5), p. 353-375, $375^{-376}$ (13. nn spp.)

I890. - 3. A new parasit Copepod. With. I pl. - Trans. N. Zealand Inst. Vol. 23 (6), p. $227-229$.

Sopra il Lepeophtheirus Erecsoni.

1889. Thompson I. C., 1. Third rep. on the Copepoda of Liverpool Bay. - Proc. Liverp. Biol. S. III.

1893. - 2. Report Copep. of Liverp. Bay. - Trans. Liverp. Biol. Assn. pl. XXIV.

19oo. Thompson I. C. and Scott A., 1. Some recent Additions to the Copepoda of Liverpool Bay. - From Trans L'pool. Biol. Soc. Vol. XIV.

E un elenco di copepodi fra cui trovansi cinque parassiti. Questo lavoro ha valore faunistico.

1903. - 2. Supplementary Report VII on the Copepoda. - Report to the government of Ceylon on the Pearl Oyster fisheries of the gulf of Manaar, published by the Royal Society.

In ultimo dell' elenco che comprende nomi di forme libere, sono indicati alcuni copepodi parassiti, fra cui una specie nuova che viene descritta dagli autori. Questa è il Chondracanthus cynoglottidis, trovata nel ceco nasale di Cynoglossus brachyrbynchus e C. brevirostris, da J. Johnstone.

Naturalmente tutti i copepodi fanno parte della fauna di Ceylon.

I896. Thomson J. S., A preliminary Notice of a parasitic Cepepod from the vas deferens of Nephrops norvegicus in: Proc. R. Phys. Soc Edinb. Vol. I3, p. 246-250.

L' autore tratta di un copepode indeterninato prossimo dell'Anchorella Triglae Cls. (e probabilmente della famiglia dei Lernaeopodidae) che è stato trovato nel canale deferente di Nephrops norvegicis.

I900. THOR SIG, Description préliminaire d'une nouvelle espèce du genre Sphyrion Cuv. (Sphyrion australicus n. sp.) d'Australie, comparée à Sphyrion laevis Quoy et Graimard. - Ann. Sc. Nat. Zool. (8), T. II, n. 2/6, p. 277, 281-282. (Les deux planches ne sont pas publiées). 
1859. ThORELL T., 1. Till Kännedomen om vissa parasitiskt lefvande Entomostraceer. - Oefvers. Vetensk. Akad Förhandl. XVI, p. 335-362. Ins Deutsçhe übersetzt: Zeitschr. f. die gesammt. Naturwiss. XV, p. II 4-I 43 .

1860. - 2. Bidrag till Kannedomen om Krustaceer, som lefva i arter af slägtet Ascidia I. - Kongliga Svenska Vetenskaps Akademiens, Handlingar (new series), III, n. 8, 84 pag., c. tab. I4.

L'aut. in questo lavoro descrive i seguenti copepodi:

r. Fam. Notodelphydae: Notodelphys Allmanni, rufescens, tenera, crerulea, elegans, agilis e prasina; Doropygus pulex, psyllus, auritus, e gibber. Botacbus cylindratus e Ascidicola rosea.

2. Fam. Buproridae: Buprorus Loveni.

3. Fam. Sapphirinidae: Lichomolgus albens, marginatus, forficola e furcillatus.

4. Fam. Ascomyzontidae: Ascomyzon Lilljeborgii.

Tutte le specie sono descritte come nuove. Furono raccolte nell'Ascilia venosa, parallelogramma, aspera, conina, mentula, intestinalis; Cynthia rustica, lurida n. sp. e tesselata.

Alcune sono vere parassite altre semi-parassite e abitano in parte la cavità branchiale o anche lo spazio tra le foglioline branchiali delle summentovate Ascidie.

$\dot{E}$ in questo lavoro che Thorell dà un prospetto sistematico dividendo $\mathrm{i}$ copepodi in 3 serie parallele:
I. Gnathostoma
II. Pœcilostoma
Ascomyzontidae
Nicothoidae
III. Siphonostoma Dalelestidae
( $\begin{aligned} & \text { Caligidae e } \\ & \text { Pandaridae }\end{aligned}$
Lernaeopodidae
Lernaeidae

1894. Trmm R., Copepoden und Cladoceren der östlichen und sydöstlichen Nordsee in Wissenschaftliche Meeresuntersuchungen. (Kiel und Helgoland). - Neue Folge, Erster Band, Heft.

1862. TURner W. and WiLsoN H.S., Observations on the parasitic Crustacea Chondracanthus lophii and Lernaeopoda Dalmanni. - Transact. of the Royal Soc. of Edinburgh, XXIII, p. 67-86, p1. 4.

1807. Turton W. M. D., British Fauna, I, I 37, n. I05, n. Io8.

L'a. descrive Lernaea encrasicoli Turton e la Lemaea cyprinacea.

1878. VALLE, 1. Sopra due specie di crostacei parassiti dell' Oxyrhina Spallanzanii Raf. - Estr. dal Boll. delle Scienze Nat., n. x, Ann. IV.

L'autore descrive l'Anthosoma Snithii Leach. e la varietà della Nemesis mediterranea da lui distinta coll' aggettivo di sinuata. Ambedue questi copepodi parassiti egli li indica pell'Adriatico, ma non sono esclusivi a questo mare soltanto.

1880. - 2. Sopra una specie nuova del genere Stellicola Ksm. - Estr. dal Boll. delle Scienze Nat. in Trieste, Vol. VI, fasc. I.

L'autore descrive una specie nuova ( $\%$ ) di Stellicola Kossmann, a cui ha dato il nome di Stellicola Kossmanniana Valle, specie trovata sopra di un bellissimo esemplare del Pleraides griseum Tongespinosum Klk., raccolto nel golfo di Costantinopoli. 
1880. VAlle A., 3. Crostacei parassiti dei Pesci del mare Adriatico. - Boll. Soc. Adriat. Sc. Nat. Vol. VI, p. 55-90.

Ė un elenco di 70 copepodi parassiti viventi nell'Adriatico. Ogni nome é accompagnato dalla sinonimia e relativa esposizione bibliografica. L' autore stabilisce tre specie nuove, ma non le descrive:

Chondracanthus Levirajae sp. n. - Brachiella oblonga sp. в. Valle - Philichthys Richiardii sp. n.

I882. - 4. Aggiunte ai Crost. parass. dei pesci del mare Adriatico. - Estr. dal Boll. Soc. Adriat. Sc. Nat. Trieste, Vol. VII (3 p.)

In questo lavòro l'autore aggiunge il nome di 7 altre specie di copepodi parassiti fra cui cita soltanto le nuove specie seguenti, ma non le descrive:

Lepenphtheirus Trigonis Valle - Clavella Sargi Valle.

I884. - 5. Seconda serie di Aggiunte al catal. Crost. parass., ecc. - Estr. Atti Museo Civ. Stor. nat. Trieste. Vol. 7, I884 (3 p.) VII a 6 sp., 2 e I n. sp. (senza descriz.)

Come dal titolo, l'autore dà l'elenco di 6 altre specie in aggiunta a quelle pubblicate nel suo primo catalogo. Qui pure cita l'Eucanlbus Marcheselti sp. n. senza descriverla.

1900. VANey C. et Conte A., Sur un Chondracanthide nouveau parasite du Clinus argentatus Riss. (Diocus clini). - Ausz. von F. Zschokke, Zool. Centralbl. 7. Ihg., n. 24/25, p. 897. - Abstr. Journ. R. Mier. Soc. London, 1890. P. 6, p. 67I (Revue Suisse Zool.) - Extrait de la Revue Suisse de Zoologie, T. 8, I900, avec la planche IO. Genêve.

Gli autori descrivono la femmina e il maschio allo stato adulto e i primi stadi larvali del detto n. copepode. Riferiscono circa l'azione del parassita sull'ospite: avendo constatato che i Clinus con parassiti avevano organi genitali molto rudimentali ne deducono che si tratti di un caso di castrazione parassitaria analoga a quella che subisce la sardina sotto l'azione del Peroderma cylindricum. Inoltre essi notano che questi parassiti sono localizzati essendo stati trovati esclusivamente nei Clinus presi nella rada di Tolone.

I87 ?. VejDovskÝ FR., 1. O samečku etc. (Uber das Männchen von Lernaeopoda selachiorum - Böhmisch) - in: Anzeig. 2. Vers. Böhm. Arzte u. Naturf., p. 58 .

1877. - 2. Untersuchungen über die Anatomie und Metamorphose von Tracheliastes polycolpus Nordm. -- Zeitschr. für Wiss. Zool., t. XXIX.

1846. Verany G. B., Catalogo di Crostacei. - Descriz: di Genova e del Genovesato, Vol. I, p. 89. Genova.

E il primo elenco sistematico di crostacei liguri, catalogo non poco importante per quel tempo, in cui, accanto ad un numero discreto di crostacei superiori (Decapodi) troviamo elencate le specie seguenti di cop. par. :

1. Caligus Rissoanus M. Edw. - 2. Cecrops Latreilli - 3. Nemesis Carcherium Brun. - 4. Brachiella Thynni Cuv. - 5. Penellus filosus M. Edw. - (Penella filosa Cuv,, Lerneopenna Blainv.) 
1845. VOGT C., 1. Beiträge zur Naturgeschichte der Schweizerischen Crustaceen. - Neue Denkschr. der allg. Schweiz. Gesellsch. für die gesammten Naturwissensch., VII.

I877. - 2. Recherches cotières faites à Roscoff; Crustacés parasites des Poissons. Avec 6 pl. Genêve. H. Georg. I877, p. I04 - oppure: Arch. de Zool. Exp. et Gén. VI, 1877, p. 385-456.

É uno tra i lavori recenti più ben fatti 'e che contribuirono assai alla conoscenza morfologica e anatomica dei cop. par. L' a. in una prima memoria tratta della fam. dei Philichthydi e in particolare del Léposphilus Labrei Hesse. Parla anzitutto della sua condizione d'esistenza e della sua abitazione. Descrive il maschio e quindi la femmina e ne stabilisce il posto nella sistematica tra i Philicl.thys di cui dà la descrizione del Ph. Xiphiae maschio per mostrare l'analogia di forma col Leposphilus.

L'a, ascrive anche i Colobomati di Hesse (1873) fra i Philichlhyidae e caratterizza questa famiglia. Accompagna le sue descrizioni con 2 tavole e molte figure.

In una seconda memoria l'a. tratta della famiglia dei Lernaeopodidae e con riguardo speciale ai maschi pigmei. Descrive il gen. Brachiella e la specie $B$. malleus Rudolphi $c$ e $q$ ed enumera tutte le specie fino allora note di questo gen. Passa poi a descrivere il gen. Anchorella sia nella forma maschile sia in quella femminile, facendo il nome delle specie note; e il gen. Charopinus ed altri generi facendo uno studio sulla omologia delle appendici fra $c^{\prime}$ e o di tutta la famiglia, della quale dà i caratteri distintivi. Lo stesso studio è fatto dall' autore per la fam. Choniracanthidae di cui stabilisce la vera posizione sistematica nell' ordine naturale. Questa seconda memoria è accompagnata da 4 tavole.

1878. - 3. L'adaptation des Crustacés copèpodes au parasitisme in: Act. Soc. Helvét. Sc. Nat. Besc. $60^{\mathrm{e}}$ e Sess. (1877) I878, p. I 2 I-139. - Revue Scientif. 2 Sér., T. I3, I877, p. 337-342.

1891. VoIgt W. D.r, 1. Synapticola teres n. gen. n. sp. ein parasitischer copepode aus Synapta Kefersteinii Sel. - Abstr. in: Journ. R. Micr. Soc. London, I892. P. 4, p. 479-480. (Bonn. I891, Zeitschrift f. Wiss. Zoologie, Bd. III. Suppl.)

1903. - 2. Beiträge zur Kenntniss des Vorkommens von Fischparasiten in den Plöner Gewässern. - Forschungsberichte aus der Biologischen Station zur Plön, Theil X.

1902. Wesenberg-Lund C., Sur l'existence d'une faune relicte dans le lac de Furesö. - Oversigt over det Kgl. Danske Vidensk. Selsk. Förh. n. 6.

1848. WILL F., Ueber Staurosoma, einen in den Actinien lebenden Schmarotzer. Wiegmanns's Archiv. f. Naturgesch. X, I, p. 337-342.

L'autore descrive il nuovo genere di copepode Staurosoma, del quale oggi non si conosce, ancora, con esattezza la posizione sistematica, seppure venga ascritto provvisoriamente tra i Chondracanthini.

1905. Wilson CH. BR., 1. North American Parasitic Copepods belonging to the Family Caligidae. Part. I: The Caliginae. - Proc. U. S. Nat. Mus. Vol. 28, p. 479-672, 25 pls., 50 figg.

(17 nn. spp. in: Caligus 8, Caligodes, Lepeophtheirus 7, Homoiotes n. g.) 
190.5. WilsON CH. BR., 2. New Species of Parasitic Copepods from the Massachusetts Coast. Proc. Biol. Soc. Washington, Vol. I 8, p. I 27-I3I.

(5 nn. spp. in: Gloiopotes, Alebion 2, Nesippus, Eudactylina).

1905. - 3. A Problem in Degeneration (Parasitic Crustacea). - Amer. Soc. Zool.; Science N. S., Vo1. 21, p. 376-377.

1877. WIERZEIJSKI, Ueber Schmarotzer-Krebse von Cephalopoden. - Zeitschr. f. Wiss. Zool., Bd. XXIX.

In questo importante lavoro, nel primo paragrafo, l'autore fa conoscere un fatto essenziale del parassitismo di certe lernee (Pennella varians St. et Lt. ?). Nella loro giovine etá, allo śtato di larve, esse vivrebbero temporariamente sopra di un ospite diverso da quello che sceglieranno di poi nell' età matura. La Pennella varians St. Ltk. da vecchia parassita de! pesce Coryphaena, albergherebbe allo stato di larva su cefalopodi (Sepia officinalis, sul Loligo vulgaris, Eledone moschala): nello stesso modo che la Lernaea branchialis per le ricerche di Metzger e di Claus fu trovata allo stato giovanile parassita temporanea del Platessa flesus e del Cycloplerus lumpus, e piu tardi invece su di altri ospiti.

L' autore, nel secondo paragrafo, descrive il Lichomolgus sepicola Cls. parassita sulle branchie di Sepia officinalis.

Il lavoro è accompagaato da tre buone tavole.

1870. Wright Perceval Edw., On a new species of the genus Pennella. With a Plate. - From the Annals and Magazine of Nacural History for January.

L'autore presenta un' introduzione in cui tratta del posto sistematico del gen. Pennella Oken e quindi descrive la Pennella Orthagorisci a. sp. e ne dà, in una tavola, il disegno.

1882. Wright Ramsay R., I. Notes om American parasitic Copepoda, I. Toronto. Reprinted from Proceedings Canadian Institute, N. S., Vol. I, n. 3, pp. 243-254. December, 1882, Plate I, II.

L'autore tratta di tre specie di cop. par. dei pesci d'acqua dolce, ma viventi in America e le descrive in modo completo, corredando la descrizione con figure distribuite in due tavole: Ergasilus centrarchidarum n. sp. - Lernaeopoda Edwardsii Olsson - Achtheres micropteri n. sp.

1885. - 2. On a parasit Copepod of the Clam (Myicola metisiensis). - Amer. Naturalist, Vol. XIX.

1854. ZENKER, System der Crustaceen. - Archiv. f. Naturgesch. XX, I.

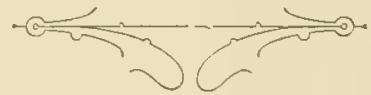




\section{ELENCO ALFABETICO DEI PESCI D'ITALIA}

\section{che furono trovati affetti da copepodi parassiti.}

Acanthias vulgaris Risso,

Trebius caudatus KRöY.

Eudactylina acuta v. BENED.

Acipenser Nacarii Bp.

Dichelesthium Sturionis HERM.

Acipenser sturio LINN.

Dichelesthium Sturionis HERM.

Alburnus alborella de FiL.

Lamproglena pulchella NordM.

Alburnus sp.

Lamproglena pulchella NORDM.

Alopias vulpes LINN.

Nesippus orientalis HelL.

Dinematura latifolia STP. et LüTK.

Nemesis lamna Risso,

N. robusta v. BENED.

Asterodermus coryphaenoides ( ${ }^{1}$ ),

Bomolochus cornutus Ciaus,

Lütkenia Asterodermi Ciaus,

Atherina Boyeri Risso,

Periculus fistula NordM.

Balistes sp.

Eucanthus Balistae Claus,

Balistes eaprisens LINN.

Eucanthus Balistae Claus,

Belone acus Risso,

Bomolochus Belones BuRM

Belone rostrata $F_{A B}$.

Bomolochus Belones BuRM

Belone vulgaris FLEM.

Bomolochus Belones Burm

Box boops Lins.

Polyrrhynchus Sieboldir Rich.

Lernaeenicus Labracis RıcH.

Naobranchia cygniformis HESSE,

(1) A. elegans Risso, stadio giov. di Luvarus imperialis RAF.
Box salpa LinN.

Caligus ligusticus n. sp. mihi,

Polyrrhynchus Richiardi VaLLE,

Clavella sp.

Brama Rayi Bl., SchN.

Polyrrhynchus HaEkeIII Rich.

Brachiella insidiosa HeLler?

Cantharus lineatus MONT.

Clavella macrotrachelus mihi?

Cl. Canthari Rich.

Cantharus orbicularis Cuv, e VAL.

Clavella Canthari Rich.

Carcharias Iamia Risso,

Caligus Petersii Rich.

Perissopus dentatus STP. et LüTk.

Carcharodon Roudeletii M. et $\mathrm{H}$.

Dinematura latifolia STP. et LüTK.

Echtrogaleus coleoptratus STP. et LüTK.

Nemesis lamna Risso.

Centrophorus granulosus BLoch,

Echthrogaleus coleoptratus STP. et LüTK.

Cerna aenea,

Hatsckekia Cernae GoggIo,

Cernas gigas $\mathrm{Bp}$.

Hatschekia Cernae Goggio,

Cycnus gracilis M. EDw.

Charax puntazzo LinN.

Lernanthropus vorax RICH.

Polyrrhynchus Agassizii RICH.

Clavella Characis RicH.

Chloropthalmus Agassizii BP.

Clavella Denticis KRöy.?

Chrysophrys aurata LINN.

Caligus productus DANA,

Polyrrhynchus Baraldii Rich.

Claper alosa Cuv.

Pseudoeucanthus Alosae Brian, 
Clupea finta Cuv. (Alosa vulgaris VAL),

Caligus minimus OтTо, Clavella emarginata KRöy.

Clupea papalina $\mathrm{BP}_{\mathrm{P}}$

Bomolochus cornutus CLAUS,

Clupea sardina RIsso,

Bomolochus cornutus Claus,

Lernaeenicus sprattae Sow.

Peroderma cylindricum HELLER,

Conger vuIgaris Cuv.

Cycnus pallidus v. BENED

Corvina nigra Cuv.

Lernanthropus Gisleri v. BENED.

Sphaerifer Corvinae LEYD.

Lernaeenicus vorax Rich

Clavella hostilis Heller,

Coryphaena hyppurns LinN.

Caligus Coryphaenae Sr. LüTK.

Coryphaena pelagica LAC.

Caligus Coryphaenze ST. LüTK.

Crenilabrus pavo $C$. V.

Hatschekia Damianii Brian,

Hatschekia sp. n.?

Cyprinus sp.

Ergasilus Sieboldii Nordm.

Lernaeocera cyprinacea LiNN

Dasybatis clavata Buv.

Charopinus Dalmannii RE'Tz

lentex gibbosus RafN.

Caligus vexator HeLLER,

Dentex rulgaris Cuv. e VAL.

Caligus vexator Heller,

Polyrrhynclus Denticis Rich.

Clavella fallax HeLt.

Dicerobatis giornae GunT.

Diphyllogaster Thompsoni BRIAN,

Esox lucius LinN.

Lernaeocera esocina BuRM.

Exocoetus Rondeletii C. V.

Bomolochus cornutus Ceaus,

Exocoetus volitans LINN.

Bomolochus cornutus CIAUS,

Galeus canis LINN.

Lütkenia integra RICH.

Pandarus bicolor LEACH,

Perissopus dentatus STP. et LüTK.

Kröyeria lineata v. BENED.
Gobius capito Cuv. et VAL.

Anchistrotos Gobii BrIAN,

Gohins flnriatilis Cuv.

Lernaeocera cyprinacea LINN.

Gobius joze LinN.

Chondracanthus horridus HeLLER,

Gobius Panizzae Verga,

Chondracanthus Ninnii Rich.

Lahrax Inpas Cuy.

Caligus minimus OTro,

Lernanthropus Kröyeri v. BENED.

Lernaeenicus Labracis Rich.

Brachiella insidiosa HELLER?

Clavella laciniata $\mathrm{K}_{\mathrm{R}}$. ?

Labrus sp.

Hatschekia Damianii Brian,

Labrus festivus Risso,

Hatschekia Richiardii Gogglo,

Labrus merula LrNN.

Hatschekia Richiardii Goggio,

Labrus turdus BL.

Polyrrhynchus Doderleini Rict.

Laeviraja maerorhmehns BP.

Charopinus Dalmanni Rerz.

Laeviraja oxyrhynchus BP.

Nemesis robusta $v$. BENED.

Chondracanthus annulatus OLss.

Charopinus Dalmanni Retz.

Lamna cornubica LINN.

Dinematura producta STEENsTr. et Lütr.

Nemesis lamna Risso,

Lepidopns candatus Eupar.

Caligus Lepidopi Rica.

Lichia glauca LINN.

Bomolochus unicirrus Rict.

Brachiella elegans RICH.

Clavella Lichiae Rich.

Lichia amia LINN.

Bomolochus unicirrus RicH.

Caligus Lichize n. sp.

Lernanthropus Gisleri v. BENED.

Nemesis lamna RIsso,

Polyrrhynchus Lichiae RıcH.

Lernaeenicus gracilis HELLER,

Brachiella elegans Ricr.? 
Lophius budegassa SPIN.

Cycnus? budegassae $\mathrm{K}_{\text {RöYER, }}$

Lophius piscatorius LINN

Chondracanthus Lophii JoHNST.

Luvarus imperialis RAF.

Lütkenia Asterodermi CLAUS,

Macrourus coelorhynchns Risso,

Rebelula Edwardsii KöıI.

Maena vulgaris Cuv. et VAL.

Lernaeolophus sultanus NORDM.

Clavella clava Rich.

Merlucius sp.

Clavella uncinata MüLLER,

Merlucius esculentus Risso,

Lernaea branchialis LINN.

Chondracanthus Merlucii KRöY.

Brachiella insidiosa Helz.

B. Merlucii BASS.-SM.

Clavella crassa RicH.

Cl. simplex Rıch.

Micropteryx Dumerili Risso,

Lernanthropus Micropterygis Rici.

Motella tricirrata BLock,

Eucanthus Marchesetti VALLE,

IIngil amratus Risso,

Ergasilus nanus v. BENED.

Lernanthropus Mugilis BRIAN,

Lernaecnicus neglectus RICH.

Mrgil enpito Cuv.

Ergasilus nanus v. Bened.

Lernaeenicus neglectus Rich.

Mngil cephalus Cuvi.

Ergasilus nanus v. BENED.

Caligus curtus MüLLER,

Lernaeenicus neglectus Rich.

Brachiella oblonga $V_{A L L E}$,

Mngil chelo Cuv.

Lernaeenicus neglectus Ricr.

Mugil saliens Risso,

Ergasilus nanus v. BENED.

Lernaeegicus neglectus Rich.

Brachiella oblonga VALIE,

Mnlins barbatus LinN.

Hatschekia Mulli v. BENED.

Polyrrhynchus Steenstrupii RicH.
Muraena helena LINN.

Bomolochus Muraenae RICH.

Hatschekia obesa RICH.

Polyrrhynchus Muraenae RICH.

Mullus surmaletus LrNs.

Hatschekia Mulli v. BENED.

Polyrrhynchus Steenstrupii RicH.

Mnstelus equestris Bp.

Lütlenia integra Rich.

Perissopus dentatus STP. et LüTK.

Kröyeria lineata v. BENED.

Nemesis robusta v. BENED.

Tripaphylus Musteli v. BENED.

Achtheres Galei KröY. (A. Selachiorum KuRZ;.

Mustelus laevis Risso,

Nesippus orientalis HeLLER,

Perissopus dentatus STP. et LüTK.

Achtheres Galei KröY.

Mastelus plebejus BP.

Perissopus dentatus STP. et LüTK.

Nemesis robusta v. BENED.

Myliobatis aquila LINN.

Achtheres Galei KRöY.

Brachiella pastinacae v. BENED.

Myliobatis noctula Bp.

Brachiella malleus Rud.

Naucrates ductor RAF.,

Pennella crassicornis STP. et LüTK.

Nettastoma melanura RAF.

Lernaeenicus sp.

Notidanus griseus Cuv.

Caligus (ß) Lessonianus RIsso,

Nemesis robusta $\mathrm{v}$. BENED.

Oblata melanura Cuv.

Bomolochus oblongus RicH.

Lernanthropus brevis Rich.

Odoutaspis ferox Agass.

Nemesis lamna Risso,

Orthagoriseus molae LINN.

Lepeophtheirus Nordmanni M. EDw.

Philorthagoriscus serratus HoRST,

Cecrops Latreillii LEACH,

Orthagoriscicola muricatus KRöY.

Pennella rubra n. sp. mihi, 
Oxyrhina Spallanzanii BONAP.

Dinematura latifolia STP, et IŨTK.

Pandarus lugubris Heller,

Anthosuma crassum ABILD.

Nemesis lamna Risso,

N. lamna var. sinuata VALLE,

Pagellus acarne Cuv.

Caligus diaphanus NordM.

Pagellus centrodontus Cuv. et VAL.

Hatschekia Pagelli-bogneravei HESSE,

Pagellus erythioinus Cuv.

Caligus diaphanus NORDM.

Polyrrhynchus Pagelli Rich.

Brachiella exigua n. sp. mihi,

B. minuta Rich.

Clavella Pagri Kröy.

Cl. Pagelli Kröx.

Cl. strumosa n. sp. mihi,

Cl. tenuis Rich.

Pagellus mormyrus Cuv.

Caligus diaphanus NoRDM.

Polyrrhynchus Pagelli Rich.

Clavella Pagelli KRöy.

Pagrus vilgaris Cuv, et VaL.

Caligus vexator HELL.

Polyrrhynchus Pagri Rich.

Clavella Pagri Kröy.

Naobranchia cygniformis HESSE,

Pelamys sarda $\mathrm{BL}$.

Caligus Pelamydis Kröy.

Perea sp.

Achtheres Percarum NoRdm.

Phicis blennioides BL. ScHN.

Clavella alata mihi,

Platessa passer Bp.

Caligus diaphanus NordM.

Lepeophtheirus pectoralis NORDM.

Pleuronectes sp.

Chondracanthus cornutus MūLLER,

Pleuronectes Grohmanni HECK.

Lepeophtheirus Grolımanni KRöY.

Prionodon glanens LINN.

Dinematura latifolia STP. et LüTK.

Phyllophora crassa RicH.

Pandarus bicolor LEACH.

Kröyeria aculeata GERST.

Raja macroryuchus $\mathrm{R}_{\mathrm{AF}}$ ?

Trebius caudatus $\mathrm{K}_{\mathrm{R} O \mathrm{y}}$.
Raja maculata MoNT.

Brachiella inconcinna RicH.

Rhinoptera marginata $\mathrm{M}$. $\mathrm{H}$.

Brachiella pastinacae v. BENED.

Rhombus laeris ROND.

Lepeophtheirus Thompsoni BAIRD,

Rhombus maximus Cuv.

Lepeophtheirus Thompsoni BAIRD,

Lepeophtheirus pectoralis v. NORDM.

Sargus annnlaris CUV. et $V_{A L}$.

Lernanthropus brevis RICH.

Hatschekia Sargi VALLE,

Polyrrhynchus Grubeii RICH.

Clavella Sargi Kurz,

Cl. elongata Rich.

Naobranchia cygniformis HESSE,

Sargus Rondeletii $C_{A v}$. et VAL.

Caligus ligusticus n. sp, mihi,

Lernanthropus brevis RICH.

Hatschekia Sargi VALLE,

Clavella macrotrachelus mihi,

Nabranchia cygniformis HESSE,

Sargus Salvianii Cuv.

Lernanthropus brevis Rich.

Hatschekia Sargi VALLE,

Peniculus fistula NordM.

Lernaeenicus Sargi RıcH.

Clavella Sargi Kurz,

Sargus rulgaris GoEFF.

Lernanthropus brevis RicH.

Clavella Sargi Kurz,

Cl. macrotrachelus n. sp. mihi,

Sayris Camperii Lac.

Bomolochus cornutus Claus,

Scardinius erythrophthalmus Lins.

Lamproglena pulchella NoRDM.

Sciaena aquila LAC.

Lernanthropus Gisleri v. BENED.

Polyrrhynchus Sciaenae Rich.

Sphaerifer Corvinae LExDIG,

Lernaeenicus vorax Ricy.

Brachiella neglecta RicH.

Clavella Sciaenae n. sp. mihi,

Scomber scomber LinN.

Caligus Pelamydis KRôy.

Clavella Scombri Kurz,

Cl. paradoxa v. BENED. 
Scomber colias LINN.

Clavella Scombri Kurz,

Scomberesox Rondeletii C. V.

Bomolochus cornutus Ciaus,

Scopelus Benoiti Cocco,

Peroderma Bellottii RICH.

Scopelus candispinosns JoHs.

Peroderma Bellottii RICH.

Scorpaena serofa LINN.

Strabax monstrosus NoRDM.

Seyllium canienla LinN.

Achtheres Galei KR. (Lernaeopoda Scyllii Rich.)

Scyllium stellare LINN

Achtheres Galei KR. (Lernaeopoda Scyllii Rich.)

Selache maxima GüNNER,

Dinematura producta STP. et LüTK.

Serranus cabrilla LiNN.

Polyrrhynchus Edwardsii RICH.

Lernaeolophus sultanus Nordm.

Serranus gigas BRüNN.

Caligus Serrani Rich.

Serranus hepatus LINN.

Polyrrhynchus minimus Rich.

Serranus seriba LinN.

Bomolochus minimus RicH.

Lernanthropus Scribae Kröy.

Lernaeolophus sultanus NORDM.

Smaris alcedo Cuv. e VAL.

Naobranchia cygniformis HESSE,

Smaris vulgaris Cuv, et VAI.

Lernaea Ninnii RICH.

Solea vulgaris Cuv.

Bomolochus Soleae Ci.Aus,

Chondracanthus cornutus MüLIER,

Sphyraena vulgaris CUV. et VAL.

Bomolochus unicirrus RicH.

Sphyrna zygaena LiNN.

Nemesis robusta $\mathrm{v}$. BENED.

Squalius cavedanus $\mathrm{BP}$.

Lamproglena pulchella NorDM

Squnlus Milberti Bp.

Perissopus dentatus STP. et LüTKN.
Squatina angelus Dum.

Eudactylina acuta v. Bened.

Stromateus fiatola LiNN.

Polyrrhynchus Stromatei RICH.

Telestes muticellus

Lamproglena pulchella NORDM.

Telestes Savignyi Bp.

Lamproglena pulchella NORDM.

Thynuus alalonga Cuv. et VAL.

Caligus Alalongae KRöy.

Thynnus pelamys Cuv. et $\mathrm{Val}$.

Pseudocycnus appendiculatus Heller,

Thynnus thynnus White,

Elytrophora brachyptera GersT.

Cecrops Latreillii LEACH,

Pseudocycnus appendiculatus Heller,

Brachiella Thynni Cuv.

Torpedo marmorata Risso,

Brachiella malleus RudoLPHI,

Torpedo narce NARDO,

Brachiella malleus Rưd

Trachinns draco LINN.

Caligus Trachini Rich.

Trachurus trachurus CASTELN.

Caligus Trachuri Rich.

Lernanthropus Trachuri BRIAN,

Lernaeenicus Labracis RicH

Trachypterus sp.

Cáligus Trachypteri KRöY.

Trigla aspera VIv.

Caligus diaphanus NorDM.

Trigla corax Bp.

Caligus diaphanus NORDM.

Brachiella impudica NORDM.

Br. obesa Rich.

Clavella Triglae Claus,

Cl. Carusi Rich.

Trigla enenIns LinN.

Caligus diaphanus NoRDM.

Trigla lineata LINN.

Caligus diaphanus NORDM.

Medesicaste Triglarum KRöX.

Brachiella impudica NORDM.

Clavella Triglae Claus,

Cl. Carusi Ricr. 
Trygla lyra LinN.

Caligus diaphanus NORDM.

Medesicaste Triglarum KRöY.

Trigla milvus LAC.

Caligus diaphanus NORDM.

Trigla obsenra LinN.

Clavella Triglae Czaus,

Trygon pastinaca LINN.

Lepeophtheirus Trygonis VALIE,

Trygon thalassia Cou.,

Nemesis robusta v. BENED.

Tylosu'us imperialis RaF.

Caligodes laciniatus Kröy.

Lernanthropus Tylosuri Rich.

Thyrsites pretiosus Cocco,

Lernanthropus foliaceus RICH.

Umbriua cirrhosa LINN.

Caligus affinis HELLER,

Lernanthropus Gisleri v. BENED.

Sphaerifer Leydigi RIcH.

Lernaeenicus vorax Rich.

Clavella hostilis HELLER,

Umbrina cirrhosa LINN.
Uranoscopus scaber LiNN.

Chondracanthus angustatus HELIER,

Xiplias gladius LiNn.

Philichthys Xiphiae STEENST.

Pennella crassicornis STP. et LüTK.

P. filosa Cuv. (P. Costai Rich.),

Chondracanthus cornutus MüLL.

Brachiella ramosa RicH.

Zens faber LINN.

Peniculus fistula NordM.

Chondracanthus Zei DeLARoche.

\section{CETACFA.}

Delphinus sp.

Pennella varians STr. et LūTr.

Prodelphinus enphrosyne True,

Pennella crassicornis STP. et LütK.

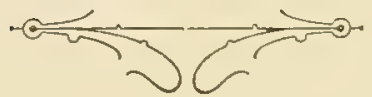




\title{
ELENCO ALFABETICO
}

\section{DEI COPEPODI PARASSITI DEI PESCI ITALIANI}

\author{
REGISTRATI NELLA SISTEMATICA
}

Achtheres Galei KRöx. . . . . . . Pag. IOI Achtheres Percarum NordM. . .. . IOI Anchistrotos Gobii n. subg, n. sp. mihi. ๖ 32 Anthosoma crassum ABILDG. . . . , 62

Bomolochus Belones Bura. . . . . y 30

Bomolochus cornutus CLaus. . . . , 3 T

Bomolochus minimus Rich. . . . . > 32

Bomolochus Muraenae RIcH. . . . , , 28

Bomolochus oblongus Rich. . . . " 》 32

Bomolochus Soleae Claus.. . . . . . 31

Bomolochus unicirrus Rich. . . . . s 30

Brachiella elegans Rich. . . . . .., 108

Brachiella exigua n. sp. mihi. . . . , 104

Brachiella impudiea NORDM. . . . , , 107

Brachiella inconcinna Rich. . . . . \$ 108

Brachiella insidiosa HeLL. . . . . \$ 104

Brachiella mallens Rud. . . ... " 103

Brachiella Merlucii B. S. . . . \ 107

Brachiella minuta RICH. . . . ., 108

Brachiella neglecta Rıсн. . . . . . \$ 106

Brachiella obesa Rich. . . . . . , in8

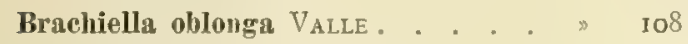

Brachiella pastinacae BEN. . . . . is 103

Bruchiella ramosa Rich. . . . . . „ 107

Brachiella Thynni Cuv. . . . . , 105

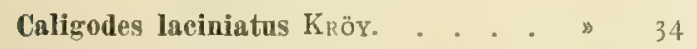

Caligus affinis HeLL. . . . . . . , 45

Caligus Alalongae KRöY. . . . . . , 37

Caligus Coryphaenae STP. et Lüтк . „ 44

Caligus cartus MüLL. . . . . . . , 35

Caligus diaphanus NordM.. . . . , 43

Cecrops Latreillii LEACH. . . . . . , 54

Caligus Lepidopi RICH. . . . . . . \$ 46

Caligus Lichiae n. sp. mihi. . . . . \$ 37

Caligus ligastieus n. sp. mihi. . . „ 4 I

Caligus minimus Отто......, 36

Caligus Pelamydis Kröy. . . . . , 43

Caligas Petersii Rich. . . . . . , 46

Caligus Pharaonis Nordm . . . . , 45

Caligus productus DANA . . . , 42

Caligus Rissonnus M. EDw. . . . . \ 37

Caligus Serrani Rich. . . . . . . 45

Caligus Trachini Rick. . . . . . , 45
Caligus Trachuri Rich. . . . . . Pag. 45

Caligus Trachypteri KröY.. . . . . , 37

Caligus rexator Helter. . . . . , 44

Charopinus Dalmanni RETZ. . . . . , 100

Chondracanthus angustatus HELI. . . " 98

Chondracanthus anmulatus OLsson.. . , 97

Chondracanthus cornutus MüLL. . . \$ 96

Chondracanthus horvidus HeLL . . . \ 97

Chondiacanthus Lophii Johnst. . . . ” 99

Chondracanthus Merlncii KRöY. . . , 97

Chondracanthus Ninnii Rich. . . . , , 98

Chondracanthus Zei Delaroche. . . , 98

Clavella alata n sp. mihi......., II4

Clavella Canthari Rich., . . . . . , 117

Clarella Carusi Rich. . . . , . . , I2I

Clavella Characis RICH. . . . . . . , II 3

Clavella clava Rıch. . . . . . . . , I2I

Clavella crassa Rich.. . . . . . . , 12 I

Clavella Denticis KröY.? . . . .. „ IIs

Clavella elongata Rich. . . . . . \ I2I

Clavella emarginata KRöY. . . . . ” IO9

Clavella fallax HéL. . . . . . " IIt

Clavella hostilis Heleer. . . . . . . Iyg

CIavella laciniata $\mathrm{K}_{\mathrm{K} O \mathrm{Y}}$ ? ? . . . . \ 120

Clavella Lichiae RICH. , . . . . . , I I I

Clavella macrotiachelus n. sp. mihi. . » II 6

Clavella Pagelli KRöy. ? . . . . \$ III

Clavella Pagri Kröy. ? . . . . . . „ I II

Clavella paradoxa BEN... . . . . „ II 8

Clavella Sargi Kurz. . . . . . . . , II3

Clavella Sciaenae n sp. mihi. . . . , 119

Clarella Scombri KuRz. . . . . . . , iा6

Clavella simplex Rich. . . . . . „ 122

Clavella strumosa n. sp. mihi. . . " 112

Clavella subtilis RicH. . . . . . , I I I

Clavella tenuis Rich. . . . . . . ๖ 122

Clavella Triglae Cla.US. . . . ., II8

Clavella nucinata MüLl. . . . . , I IO

Cyenus budegassae KRöy. . . . . \$ 74

Cyenus gracilis M. Edw. . . . . » 76

Cyenus pallidus $\mathrm{BEN}_{\mathrm{EN}} . . . ., \ldots, 75$

Demoleus paradoxus HeLLer. . . . s so

Dichelesthium sturionis HERM. . . \ 67 
Dinematura latifolia STP. et LüTK.

Dinematura producta STr. et LüTk.

Diphyllogaster 'Thompsoni BRIAN

Echthrogaleus coleoptratus STP. et LüTK.

Ergasilus nanus BeN.

Ergasilus Sieboldii NORUN

Eucanthus Balistae Claus.

Eueanthus Marchesetti VALLE

Endactylina aenta BEN.

Hatschekia Cernae Goggio . . . .

Hatschekia Damianii mihi (Clavella Acantholabri exoleti)

Hatschekia Molli BEN. . . . . . .

Hatschekia obesa Rich. . . . . . .

Hatsehekia Pagelli Bogneravei Hesse?

Hatschekin Richiadii Goggıo. . . .

Hatschekin sp. . . . . . . .

Hatschekia Sargi VALLE . . . . ,

Kröyeria aculenta GERST. . . . . , , 68

Kröyeria lineata BEN. . . . . . \$ 67

Lamproglena pulchella NORDM. . . . , 78

Lernnea branchialis LinN. . . . . " 92

Lernaeniens gracilis HeLL. . . . . » 87

Lernneenicus Labracis Rich. . . . . > 90

Lernaeenicus neglectus RicH. . . . , 88

Lernaeeniens Sargi RICH. . . . . . \& go

Lernaeenieus sp.? . . . . . . . . . 88

Lermaeenicus sprattae Sow. . . . . , 89

Lerneeanicus vorax Rich. . . . . . , 88

Lernaeocera cyprinacea LinN. . . . » 79

Lemreolophus sultanus NORDM. . . , 91

Lepeophtheirus Grohmanni KRör. . . » 48

Lepeophtheirus Nordmanni M. EDw. . „ 47

Lepeophtheirns pectoralis NORDM. . . \ 47

Lepeophtheirus Thompsoni BAIRD , . , 46

Lepeophtheirus Trygonis VALLE . . , 48

Leruanthropus brevis RicH. . . . . " 65

Lernanthropus foliaceus Rich. . . . , 66

Lernanthropus Gisleri BEN. . . . . , 65

Lernanthropus Kröyeri BEN. . . . , 》 66

Lernanthropus Micropterygis Rich. , \$ 66

Lernanthropus Mugilis BriAN. . . . , 63

Leruanthropus seribae KRöy. . . . » 65

Lernanthropus Trachuri Rrian. . . » 63

Lernanthropus Tylosuri Rich. . . . . 67

Leruanthropus vorax Rich. . . . . , 64

Liitkenia Asterodermi Claus, . . . , 48

Liitkenia integra Rich. . . . . . , 49

Medesicaste Triglarum KRöY. . . . > 94
Naobranchia eygniformis Hesse. . . Pag. 122

Nemesis Lamna Risso . . . . . . . 7 I

Nemesis (mediterranea) lamna var. sinuata VALLE, .

Nesippus orientalis HeLLER . . . . * 49

Nogagus caelebs Heller . . . . \ j0

Nogagus Latreillii LEACH . . . . , jo

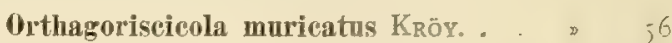

Pandarus bicolor Leach. . . . . . \& 55

Pandarus lngnbris Heller . . . . , ;6

Penicnlus fistnla Nordm. . . . . . 80

Pennella crassicarnis Str. et LütK. . , 8 I

Pennella filosa Cuv. . . . . . . , $\delta_{3}$

Pennella Remorae Murray . . . . * 87

Pennella rubra n. sp. mihi, . . . " 86

Pennella varians STP. et LüTk. . o $\delta_{2}$

Perissopus dentatus Stp. et Lürk . „ ;7

Peroderma Bellottii Rich. . . . = 94

Peroderma cylindricum HeLL. . . . , 93

Philichthys Xiphiae Steenst. . . . , j8

Philorthagorisens serratus Horst . , 33

Phyllophora erassa Rich. . . . . • 55

Polyrrhynchos Agassizii RICH. . . . , 60

Polyrrhyneus Baraldii Rich. . . . = 59

Polyrihynens Denticis Rich. . . . o 59

Polyrrhynchus Doderleini Rich. . . , 61

Polyrrhynchus Edwardsii Rich. . . \$ 6 T

Polyrihuchus Grubei Rich. . . . = 60

Polyrrhynchus Haeckelii Rich. . . , 60

Polyrrhynchans Lichiae Rick. . . . y 59

Polyrrhynchus minimus Rich. . . . s 60

Polyrrhynclus Muraenae Rice. . . . \$ 60

Polyriliynchus Pagelli Ricu. . . . o 59

Polyrrhynchus Pagri Rich. . . . . , 50

Polyrihynchus Richiardii VALLE,. . \$ 60

Polyrrhynchus Sieboldii Ricн. . . . , 60

Polyrrynchus Steenstrupii Rich. . . ๖ 6 I

Polyrrynchus Stromatei RicH. . . . , 6r

Psendocjenus appendiculatus Heller, \& $7^{6}$

Pseudoeueantlus Alosae ఇ. sp. mihi, " 26

Rebelula Edwardsii KöLı. . . . . „ 90

Sphaerifer Corrinae Leydig. . . . . 6 I

Sphaerifer Leydigii Rich. . . . . 》 62

Strabax monstrosus NORDM. . . . . 95

Trebius candatus HRöY. . . . . . , jo

Tripaphylus Musteli BEN. . . . . \$ 87

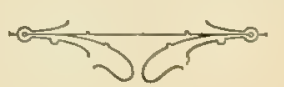




\section{SPIEGAZIONE DELLE TAVOLE}

\section{N.o 10 a colori - N. ${ }^{\circ} 11$ in nero}

\section{Spiegazione della Tavola I.}

Fig. I. Lepeophtheirus Nordmanni, femmina; ospite: Orthagoriscus mola; Napoli. (Dipinta da esemplare fresco). Lunghezza mm. IO-II.

》2. Elytroplora brachyplera, femmina; branchie di Thynnus thynnus; Napoli. (Dipinta da esemplare fresco). Lunghezza $\mathrm{mm}$. II.

》3. Stessa specie, maschio. Lunghezza mm. 8.

» 4. Philorthagoriscus serratus, femmina (vista dal dorso); ospite: Orthagoriscus mola; 3 I Luglio Igo3, Napoli. Lunghezza mm. $7^{1 / 2}$; largh. mm. 5 .

\section{Spiegazione della Tavola II.}

Fig. 1. Pandarus bicolor, femmina, sulla pelle di $\mathrm{Ga}$ leus canis; 7 Luglio 1903, Napoli. (Dipinta $\mathrm{da}$ esemplare fresco). Lunghezza, senza fili oviferi, mm. 9; lungh. dei fili oviferi mm. I7.

1) 2. Echthrogaleus coleoptratus, femmina, alla base della pinna dorsale di Charcharodon Rondeletii; 5 Giugno 1898 , Isola d'Elba. Lunghezza mm. $12 \frac{1}{2}$.

\3. Cecrops Latreillii, maschio; Napoli. (Dipinto da esempl. fresco). Lunghezza mm. 10 circa.

" 4. Stessa specie, femmina; Portoferraio, 9 Marzo 1899. Lunghezza mm. 26.

\section{Spiegazione della Tarola U.}

Fig. 1. Anthosona crassum, femmina; Portoferraio. Lunghezza del corpo senza i fili oviferi, mm. II; lungh dei fili oviferi mm. 27 .

" 2. Haischekia Sargi, femmina; Napoli. Lunghezza, senza fili oviferi, mm. 2.
Fig. 3. Hatschekia sp., femmina; branchie di Crenilabrus pavo; Io Luglio 1903, Napoli. Lunghezza, senza fili oviferi, mm, I, 6 circa ; lungh. dei fili oveferi quasi $\mathrm{mm}$. I.

》4. Masclio(?) della stessa specie. Lunghezza poco più $d i \mathrm{r} / 2 \mathrm{~mm}$.

\5. Clavella alata n. sp., femmina; ospite: Phicis bleinnoides; Napoli. Lunghezza mm. 3; senza sacchi oviferi, mm. 2.

》6. Clavella n. sp., femmina; ospite: Box Salpa; Napoli. Ingr. della figura $\times 17,5$.

\section{Spiegazione della Tavola IV.}

Fig. I. Nemesis robusta, femmina, vista dal lato ventrale; branchie di Sphyrna zygaena; I903, Napoli. Lunghezza del corpo mm. 5; lungh. dei fili oviferi mm. Iz.

» 2. Femmina vista dal lato dorsale.

》3. Post-addome della stessa.

\4. Piede mascellare del $20^{\circ}$ paio, della femmina.

5. Uova (dei fili oviferi).

» 6 Maschio di Nemesis robusta, visto da un lato.

\$ 7. Lo stesso visto dal lato dorsale. I piedi mascellari del $I .{ }^{\circ}$ paio soltanto sono segnati in questa figura e si vedono distintamente per trasparenza attraverso il tegumento.

$\gg$. Piede mascellare del $20^{\circ}$ paio, del maschio.

9. Spermatoforo.

" I0. Appendici caudali del maschio. 


\section{Spiegazione della Tavola $\mathrm{V}$.}

Fig. I. Eudactylina acuta, femmina; I903, Napoli. Lunghezza mm. 2.

» 2. Nemesis medilerr nea var. sinuata, femmina; ospite: Carcharodon Rondeletii; Portoferraio. Lunghezza mm. Io. Ingr. della figura $\times 8$.

3. Kröyeria lineata, temmina; branchie di Galeus canis; 7 Luglio 1903, Napoli. Lunghezza del corpo $\mathrm{mm}$. $5^{1 / 2}-6$; lungh. dei fili oviferi $\mathrm{mm}, 2$.

» 4. Lernanthropus Trachuri n. sp., femmina; branchie di Trachurus traclsurus; Portoferraio. Lunghezza mm. $3^{1 / 2}$

\$ 5. Stesso esemplare visto dal dorso.

\section{Spiegazione della Tavola VI.}

Fig. I. Peniculus fistula, fenmina; sull' Atherina Boyerii; 22 Luslio Igo3, Napoli. Lunghezza (senza fili oviferi) mm. 4 circa.

"2. Pennella crassicornis, femmina; infissa sulla cute di Prodelphinus euphrosyne; 3 I Luglia I902, Portoferraio. Lunghezza totale cent. 7 .

3. Cefalotorace della stessa.

》4. Regione caudale della stessa.

\$ 5. Cefalotorace di un esemplare diverso, visto dorsalmente.

"6. Lo stesso cefalotorace ingrandito visto ventralmente.

》7. Antenne uncinate della stessa Pennella.

\section{Spiegazione della Tavola VI.}

Fig. I. Bracbiella exigua n. sp. femmina; branchie di Pagellus erylhrinus; 24 Agosto Igo3, $\mathrm{Na}$ poli. Lunghezza mn. 3. Ingr. della figura $\times \quad 8,6$.

» 2. Peroderma Bellottii, femmina; ospite Scopelus caudispinosus; Lunghezza i4 $\mathrm{mm}$.

3. Pcnnella rubra n. sp. (?) molto ingrandita. Parte inferiore del corpo di una giovane femmina. Ospite: Ortbagoriscus mola; $\mathrm{Na}$ poli.
Fig. 4. Chondracanthus Merlucii, femmina; Io Luglio 1903, Napoli. Lunghezza mm. $51 / 2$.

" 5. Chondracanlbus cormulus, femmina; branchie di Xiphias gladius; 23 Gennaio I890, Portoferraio. Lunghezza mm. ${ }_{3}$.

\section{Spiegazione della Tarola VIII.}

Fig. I. Brachiella insidiosa, femmina; ospite: Merlucius esculentus Lunghezza totale del corpo mm. I 2 ; lungh dell' addome mm. 5; delle appendici addominali più lunghe mm. 4; dei sacchi oviferi mm. 7. Il cefalororace misurato trasversalmente arriva a mm. $43 / 4^{\text {. }}$

๖ 2. Brachiella malleus, femmin 3 ; mucosa boccale di una Torpedo marmorata. Lunghezza delle braccia mm. $31 / 4$; della testa e collo mm. 3 ; dell' addome mm. 4-5; dei sacchi oviferi mm. 3 .

3. Bracbiella Merlucii, femmina; branchie di Merlucius esculentus; II Gennaio Igo2, Genova.

\$ 4. Brachiella insidiosa, femmina; branchie di Merlucius esculentus; I I Gennajo 1902, Genova. I sacchi oviferi appariscono tagliati via in gran parte.

» 5. Clavella macrotrachelus n. sp., femmina; ospite: Cantharus lineatus; 3 Gennaio 1902 , Genova. Lunghezza del collo mm. 2 ; dell' addome mm. I $1 / 4$. Il maschio pigmeo è fissato all' addome della femmina.

"6. Brachiella impudica, fenumina; ospite: Trigla corax; 24 Marzo Ig04, Portoferraio. Lunghezza $\mathrm{mm}$. 8 . Ingr. della figura $\times 8$, 50 .

\section{Spiegazione della Tarola IX.}

Fig. I. Bracbiella Thynni, femmina; ospite: il tonno; 9 Gennaio Igo3, Genova. Lunghezza totale mm. 22 circa; lunghezza delle braccia mm. 5 ; dell' addome num. $61 / 2$; dei sacchi oviferi mm. เо.

» 2. Clavella Sargi, fenimina. Lunghezza mm. 4; Lunghezza dei sacchi oviferi mm. 2. Ingr. della figura $\times 12,5$.

" 3. Naobranchia cygniformis, femmina; ospite: Smaris alcedo; Portoferraio. Lungh. mm. 3. Ingr. della figura $\times 14,33$. 
Fig. 4. Clavella struniosa n. sp., femmina; branchie di Pagellus erythrinus; 2 Luglio I900, Portoferraio. Lunghezza totale mm. 6. Il corpo soltanto senza sacchi oviferi arriva a mm. $4^{1 / 2}$.

》5. Clavella strumosa n. sp., stesso esemplare della figura 4. Addome ingranditu.

" 6. Clavella strumosa n. sp., femmina; branchie di Pagellus erythrinus; 7 Novembre Igor, Portoferraio. Lunghezza del corpo, senza sacchi oviferi. mm. $31 / 2$; lunghezza dei soli sacchi oviferi $\mathrm{mm}, 21 / 2$.

» 7. Lo stesso esemplare visto da un altro lato.

\section{Spiegazione della Tavola $\mathbf{X}$.}

Fig. I. Clavella Characis, femmina; Portoferraio. Lunghezza mm. 6

* 2. Clavella Sciaenae n. sp. femmina; branchie di Scinena aquila; Ir Maggio I89I, Genova. Lunghezza del corpo senza sacchi oviferi mm. 4. Lunghezza totale, questi compresi, mm. 7 .

" 3. Clavella emarginata, femmina; ospite: Clupea finla; 6 Novembre 1889 , Genova. Lunghezza dell' addome e braccia (insieme comprese) mm. $2 \frac{x}{2}$; lunghezza dei sacchi oviferi mm. $2 x / 4$

"4. Clavella fallax, femmina; ospite: Dentex vulgaris; Portoferraio. Lunghezza senza sac-

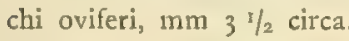

- 5. Clavella Pagri, femmina; ospite: Pagellus erylhrinus; Genova. Lungh. totale mm. 3.

6. Clavella Scombri, femmina; ospite: Scomber scombrus. Lunghezza mm. 4: lunghezza de! cefalotorace e addome compreso (misura trasversale dell' animale nella posizione in cui è disegnato) mm. 6 .

\section{Spiegazione della Tarola XI.}

Fig. 1. Pseudoeucanthus Alosae n. sp. maschio(?) visto dal lato ventrale; ospite: Clupea alosa (oculo affixa).

»2. Sacco ovifero e regione caudale della femmina.

"3. Appendici boccali del maschio(?)
Fig. 4. Antenne del $20^{\circ}$ paio dello stesso.

5. $10^{\circ}$ paio di piedi natatori 》

$\gg 6.20^{\circ}$ paio $\gg$

\7. $30^{\circ}$ paio $\gg$,

$\gg 8.40^{\circ}$ paio $\gg$

\section{Spiegazione della Tavola XI.}

Fig. 1. Bomolochus Muraenae femmina.

》2. Anello genitale e post-addome della stessa.

》 3. Parti boccali: $m d$ mandibole; $p$ palpi mascellari; $p m x^{\mathrm{I}}$ piedi mascellari del I. paio; $p m x^{2}$ piedi mascellari del $20^{\circ}$ paio.

»4. Antenne del $10^{\circ}$ paio.

》5. Antenne del 2. paio.

\6. $10^{\circ}$ paio di piedi natatori.

"7.2. paio $》 "$

8. Ramo interno del $40^{\circ}$ paio di p. n.

$9.50^{\circ}$ paio radimentale di p. n.

\section{Spiegazione della Tavola XIII.}

Fig I. Anchistrotos Gobii n. sub. gen., , n. sp. ; maschio (?). Cefalotorace visto dal lato ventrale; $a^{1}$ antenne del 1.0 paio; $a^{2}$ antenne del $20^{\circ}$ paio; $b$ hamuli laterali.

» 2. Addome e post-addome dello stesso.

\. Femmina della stessa specie.

》 4. Parti boccali della femmina; $o$ bocca; $m d$ mandibole; $p$ palpi mascellari; $p m x^{\mathrm{I}}$ piedi mascellari del $\AA^{\circ}$ paio; $p m x^{2}$ piedi m. del $20^{\circ}$ paio.

\. Sacco ovifero esterno della stessa.

»6. Antenne del $2 .^{\circ}$ paio della stessa.

》7. Antenna del I. ${ }^{\circ}$ paio del maschio.

\$8. I. paio di piedi $\gg$

9: $3 .{ }^{\circ}$ paio di piedi $\gg$

»10. $40^{\circ}$ paio di piedi $\gg$

\section{Spiegazione della Tavola XIV.}

Fig. 1. Caligus Lichiae n. sp. femmina vista dal lato dorsale.

》 2 Caligus Lichiae n. sp. femmina vista dal lato ventrale.

"3. hamulus di detto Caligus. 
Fig. 4. Bocca (succhiatoio) dello stesso.

\$5. furcula sternalis 》 》

* 6. Lunula e antenna del I. paio dello stesso.

\$7. Antenna del $20^{\circ}$ paio $\$$ »

\$8. Piede mascellare del 1.0 paio $\gg \gg$

9. I. paio di piedi $\gg$ D

\10. 2. paio di piedi $\gg$ 》

II. $30^{\circ}$ paio di piedi

"12. Ramo interno del $3 .^{\circ}$ paio di p. 》 》

$\gg 13.46^{\circ}$ paio di piedi $\gg$

\14. Post-addome e furca caudalis $\gg$ 》

\section{Spiegazione della Tavola XV.}

Fig. I. Cefalotorace di Caligus ligusticus n. sp., maschio; ospite: Box Salpa; Portoferraio. Lunghezza totale del corpo $\mathrm{mm}$. 3 .

» 2. Cefalotorace visto dal lato ventrale, di detto Caligus.

》3. Addome e post-addome dello stesso.

» 4. Antenne del I. ${ }^{0}$ paio e lunula dello stesso.

\. furcula sternalis dello stesso.

"6..$^{\circ}$ paio di piedi " "

$\gg 7 \cdot 20^{\circ}$ paio di piedi $\gg$

\$ $8.30^{\circ}$ paio di piedi $\gg$ »

\section{Spiegazione della Tavola XV.}

Fig. I. Philorthagoriscus serratus, maschio; ospite: Orthagoriscus mola, 31 Luglio 1903, Napoli. (Visto dal lato ventrale). Lunghezza mm. 5 .

» 2. Stesso maschio visto dal lato dorsale.

3. Addome dello stesso.

» 4. Cefalotorace e parti boccali e altre appendici dello stesso.

》5. Piedi mascellari del I. ${ }^{\circ}$ paio dello stesso.

\6. Appendice caudale dello stesso.

7 . 2. paio di piedi dello stesso.

》 8. Caligus Lichiae n. sp., maschio. Lunghezza mm. 4 1/2-5.

» 9. Antenne del 2. ${ }^{\circ}$ paio dello stesso.

》 ı. Piedi mascellari del 2.0 paio dello stesso.

》 Ir. Piedi del $30^{\circ}$ paio dello stesso. (Vedi la lamina mediana nel cui mezzo sono disposti organi speciali).

\section{Spiegazione della Tarola XVII.}

Fig. I. Antenne, piedi mascellari e piedi natatori di Lernanthropus Trachuri

» 2. Lernanthropus Gisleri, femmina; branchie di Lichia amia, Genova. Lunghezza senza le appendici addominali, mm. $7 \%$.

》3. Lernanthropus brevis, femmina; branchie di Sargus vulgaris, 1903, Napoli. Lunghezza comprese le appendici, mm. 2.

"4. Maschio di detta specie. Lunghezza comprese le appendici, mm. I $1 / 2-I 3 / 4$.

» 5. Antenne del $10^{\circ}$ paio del maschio di detta specie.

\section{Spiegazione della Tarola XVII.}

Fig. I. Pennella fllosa (P. Coslai Rich.), giovane femmina; ospite: Xiplias gladius, Portoferraio. Lunghezza cent. 12 .

》2. Regione caudale di detta Penuella.

" 3. Ultimo segmento del corpo di detta Pennella.

$\gg$ 4. Parte anteriore del corpo $\gg$

$a^{1}, a^{2}$ antenne; $m x$ mascelle; $p m x^{1}$ e $p m x^{1}$ piedi mascellari; $p^{1} p^{2} p^{3} p^{4}$ piedi natatori.

》) 5. Bocca (rostro) di detta Pennella

»6. Piede mascellare del primo paio di detta Pennella.

» 7. Mascella o palpo mascellare di detta Pennella.

》8. Lernaeenicus n. sp. femmina; ospite: Nettastoma melanura; Genova. Lunghezza totale mm. 25 ; lunghezza dell' addome mm. 9.

»9. Cefalotorace dello stesso, visto dal dorso; $R$ rostro, $a^{3}, a^{2}$ antenne.

\10. Cefalotorace dello stesso, visto dal lato ventraie.

» I I. Cefalotorace dello stesso, visto da un lato.

» I . Piedi mascellari di detto Lernaeenicus.

» 3 . Piede natatorio. 》

\section{Spiegazione della Tavola XIX.}

Fig. I. Il pesce Macrouras colorbynchus col parassita Rehelula Edwardsii femmina, fissato sul dorso. Lunghezza del pesce cent. 23.

* 2 Il pesce Scopelus caudispinosus col parassita Peroderma Belloltii femmina, che esce fuori dell' angolo boccale. 
Fig. 3. Cefalotorace di Peroderma Bellottii.

$a$ antenne; $m x^{\mathrm{I}}$ mascelle o piedi masc. del I. ${ }^{\circ}$ paio; $m x^{2}$ mascelle o piedi masc. del $2{ }^{\circ}$ paio; $m$ mandibole.

》 4. Appendici boccali dello stesso (come sopra).

ऽ. Antenna dello stesso.

» 6. Oestrus sive Asilus marimus, riprodotto dall'opera di Rondeletius: De Piscibus marinis ecc., p. II 2 .

\section{Spiegazione della Tavola XX.}

Fig. I. Antenne del I. ${ }^{\circ}$ paio del maschio (?) di Hatschekia sp.; ospite: Crenilabrus pavo, to Luglio Igo3, Napoli.

$\gg$ 2. Antenne del $2^{\circ}$ paio dello stesso.

» 3. Piedi mascellari del $\mathrm{I}^{\circ}$ paio dello stesso.

"4. Piedi natatori del $2^{\circ}$ paio 》 «

" 5. Clavella alata n. sp. femmina; ospite: Phicis blennioides, Napoli. Lunghezza mm. 2; coi sacchi oviferi mm. 3

》6. Parti boccali della stessa; $a^{1}$ antenne del 1. ${ }^{\circ}$ paio; $a^{2}$ antenne del $20^{\circ}$ paio; $m x$ mascelle; $m d$ mandibole; $p m x^{2}$ piedi mascellari del $2,^{\circ}$ paio.

D7. Antenne e piedi mascellari di Brachiella exigua sp. n. femmina; ospite: Pagellus erythrinus, branchie; Napoli.
Fig. 8. Estremità dell' antenna del $20^{\circ}$ paio di Clavella strumosa n. sp. femmina; ospite: $P a$ gellus erythrinus.

» 9. Piedi mascellari della stessa Clavella strumosa femmina.

》 Io. Mascelle della stessa.

\ Ir. Antenne del I ${ }^{\circ}$ paio della stessa.

》12. Estremità delle braccia con bottone chitinico della stessa.

\ 13. Mascelle di Clavella sciaenae n. sp femmina; ospite : Sciaena aquila

》14. Capo della stessa.

\section{Spiegazione della Tavola XXI.}

Fig. 1. Clavella macrotrachelus n. sp., femmina; ospite: Sargus vulgaris; 21 Luglio 1903, Napoli. Lunghezza del corpo mm. 2; lunghezza dei sacchi oviferi mm. $11 / 2$.

》2. Maschio della stessa specie, molto ingrandito.

》3. Capo della femmina e parti boccali, della stessa specie.

"4. Parte anteriore del corpo del maschio della stessa specie a fortissimo ingrandimento.

» 5. Dorso del pesce Macrourus coelorbynchus col parassita Rebelula Edwardsii; Genova.

"6. Piedi mascellari del $2 .^{\circ}$ paio di Clavella Denticis (femmina); Genova. 



\section{INDICE DEL VOLUME}

Avvertenze (Prefazione).

STORIA.

I. Copepodi lerneiformi

2. Caligidi

3. Filictidi

\section{SISTEMATICA.}

Fam. 1. Ergasilidae

Fam. II. Caligidae.

Fam. III. Philichthyidae .

Fam. VI. Dichelestbiidae.

Fam. V. Lernaeidae

Fam. VI. Chondracanthidae

Fam. VII. Lernaeopodidae

\section{COROLOGIA.}

Nizza

Genova e Liguria

Portoferraio e Isola d' Elba

Golfo di Napoli

Sicilia (senza indicazione determinata di località)

Palermo

Messina

Sardegna - Cagliari

Carloforte.

Isola di Piana.

Mediterraneo (senza indicazione determinata di località).

Adriatico - Trieste; Istria e Dalmazia .

Venezia

Rimini

Fano

Verona (Provincia) .

Italia (acque dolci) - (senza indicazione determinata di località)

Massa marittima

\section{BIBLIOGRAFIA.}

(In serie alfabetica di autori).

(A. pag. 133 - B.p. 134 - C. p. I4I - D.p.I44 - E.p.145 - F.p. I46 G. p. 146 - H. p. 149 - J. p. I53 - K. p. I53 - L. p. I55 - M. p. I58 N. p. $160-$ O. p. $160-$ P. p. $161-$ Q. p. $162-$ R. p. $162-$ S. p. $165-$ T. p. $169-$ V. p. $171-$ W. p. $173-$ Z. p. 174).

Elenco alfabetico dei pesci d'Italia che furono trovati affetti da copepodi parassiti. Elenco alfabetico dei copepodi parassiti dei pesci italiani registrati nella Sistematica Spiegazione delle tavole 



\section{ERRATA-CORRIGE}

\begin{tabular}{|c|c|c|c|}
\hline Pagina & Linea & Errata & Corrige \\
\hline 6 & I & Prefazione & Avvertenze \\
\hline $\mathrm{I}_{4}$ & 8 & Zoologia & zoologia \\
\hline 30 & 30 & rinvenne & trovò \\
\hline $3 \mathbf{I}$ & 34 & rinvenne & trovó \\
\hline 32 & 4 & rinvenne & raccolse \\
\hline 35 & $4 \mathrm{I}$ & i lavori originali & i singoli lavori \\
\hline 42 & 42 & rinvenuto & trovato \\
\hline Si & I & rinvenuta & trovata \\
\hline$\$ 5$ & I9 & rinvenne & trovò \\
\hline 56 & 15 & rinvenuto & raccolto \\
\hline 6I & 22 & Dodeeleini & Doderleini \\
\hline 87 & 9 & Remmorac & Remorae \\
\hline 105 & 27 & Brahiella & Brachiella \\
\hline I2I & I & determinato & determinati \\
\hline 122 & 9 & rinvenne & trovò \\
\hline I 28 & 47 & Dichelestium & Dichelesthium \\
\hline I3 I & 8 & Dichelestium & Dichelesthium \\
\hline I3 $\mathrm{r}$ & 27 & Peunella & Pennella \\
\hline 132 & $\mathrm{I}_{4}$ & Dichelestium & Dichelesthium \\
\hline 132 & 19 & Actheres & Achtheres \\
\hline$x 12$ & 23 & Questi esemplari che non & Questi esemplari non \\
\hline II 2 & 23 & data da Kröyer, & data da Kröyer: \\
\hline 119 & 22 & Clavella Sciaene & Clavella Sciaenae \\
\hline
\end{tabular}



T A VOLE 


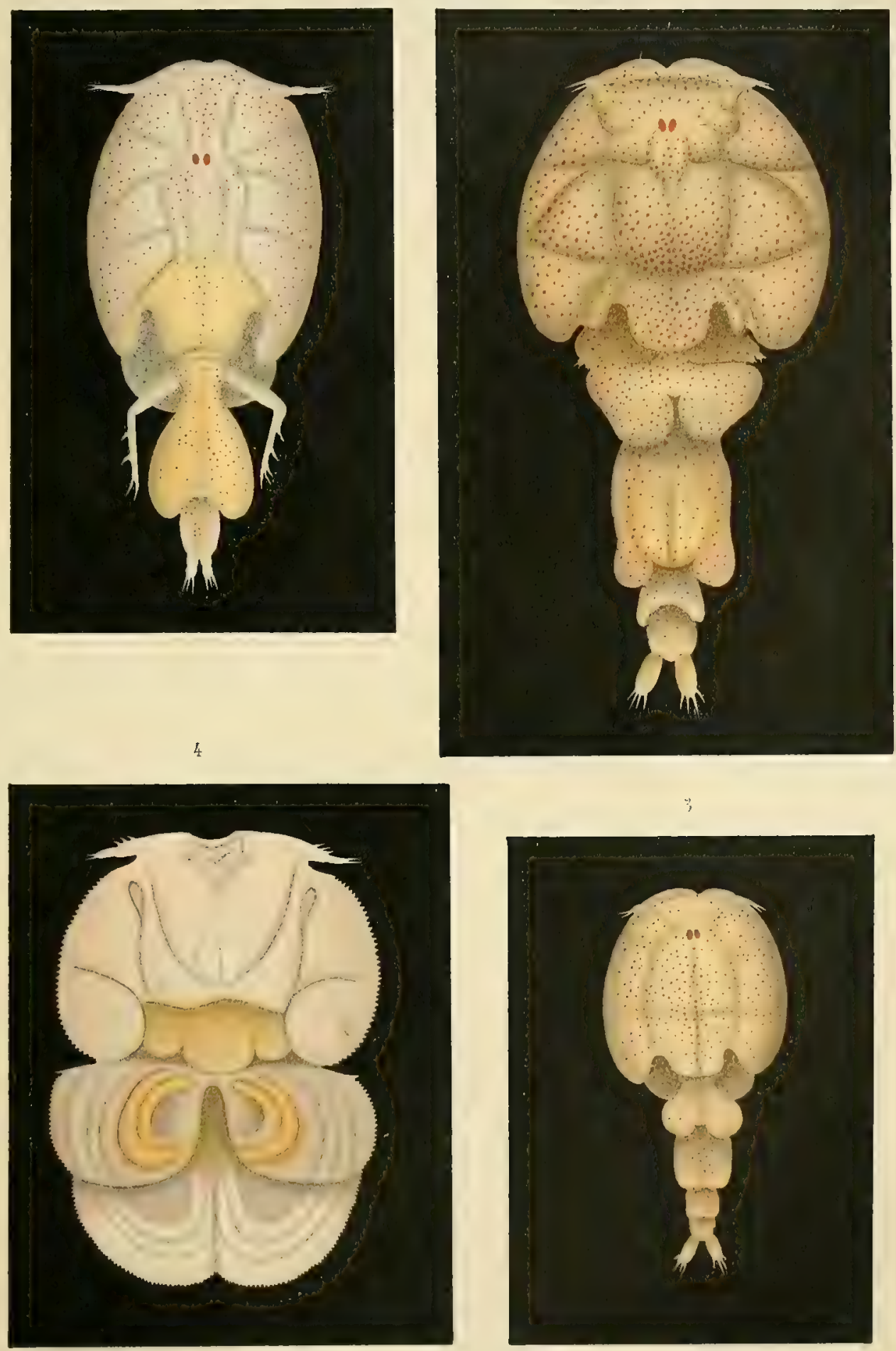

1. To epeophtheuris nordmanni. ㅇ․ 2. Elytrophora frachyptera. 우 3. idem - or

4. Philorthagoriscus serratus. I 



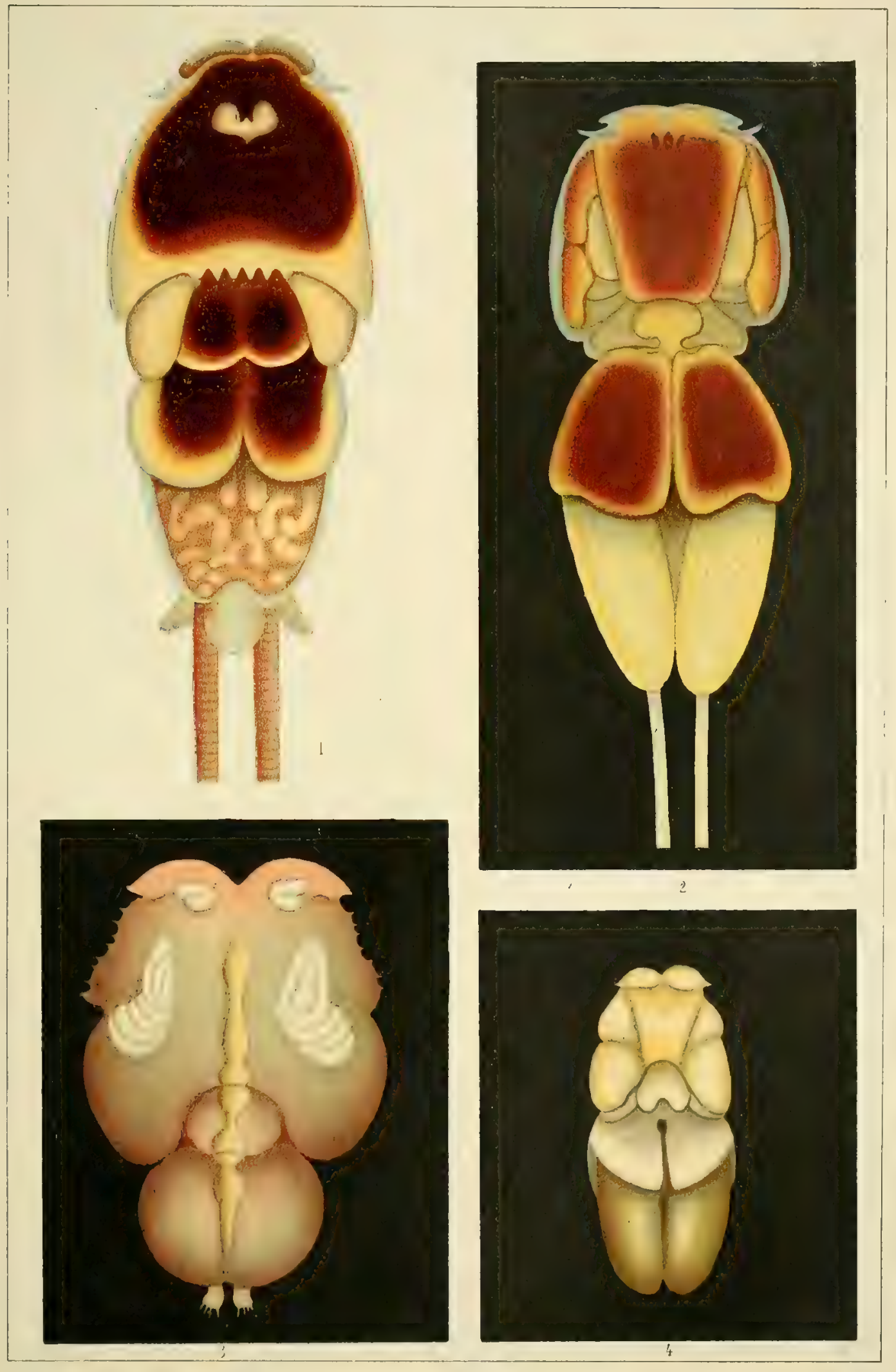

1. Pandarus bicolor. ot.

2. Echthrogaleus coleoptratus: + .

3. Cecrops latreillie. $\pi$.

4. ideun - ? 


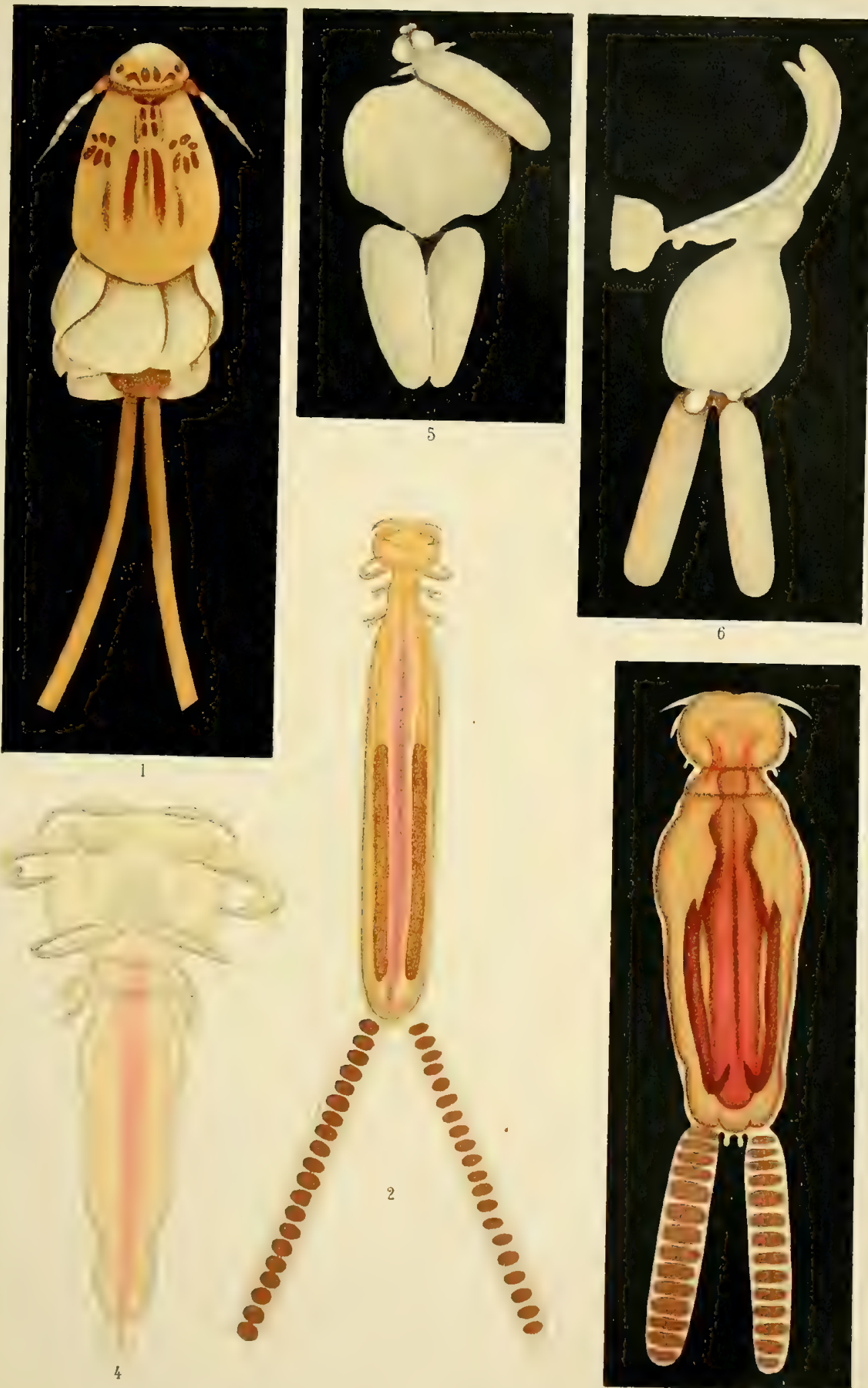

3

1. Anltasoma crassum it.

2. Hatschekia surgi. ㅇ.

3. Hatschelcia sp. + .

4. idem - ग. (?)

5. Clavella alata of.

$\because x, \operatorname{sp} q$. 



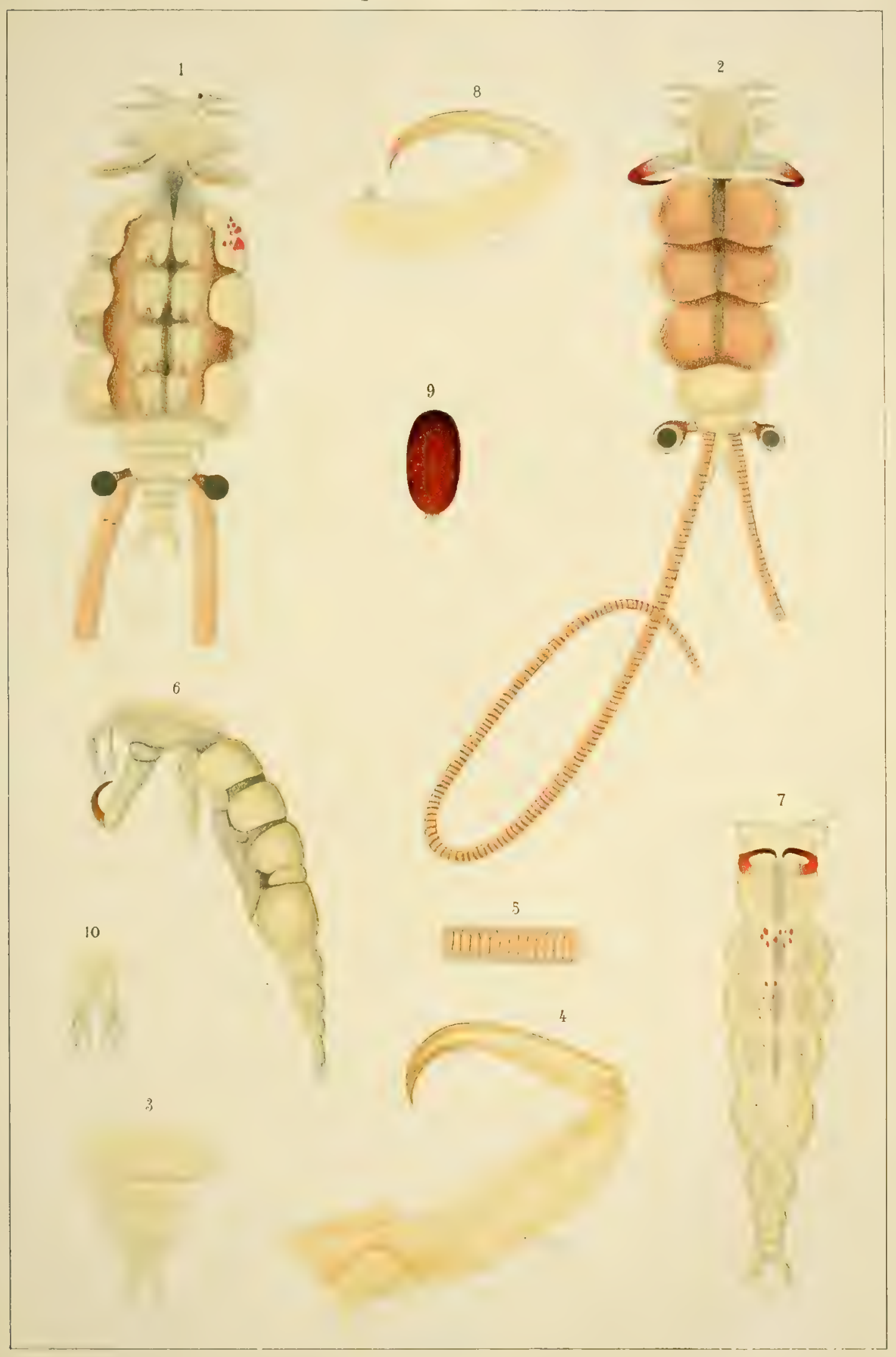

Lit Tacehinarai o Ferrast-Pavia.

1 Nemesir robusta. \&, vertral.

2. idena - dosoal.

6 - idem $\pi$, lateral.

T-idem q. dorsal. 



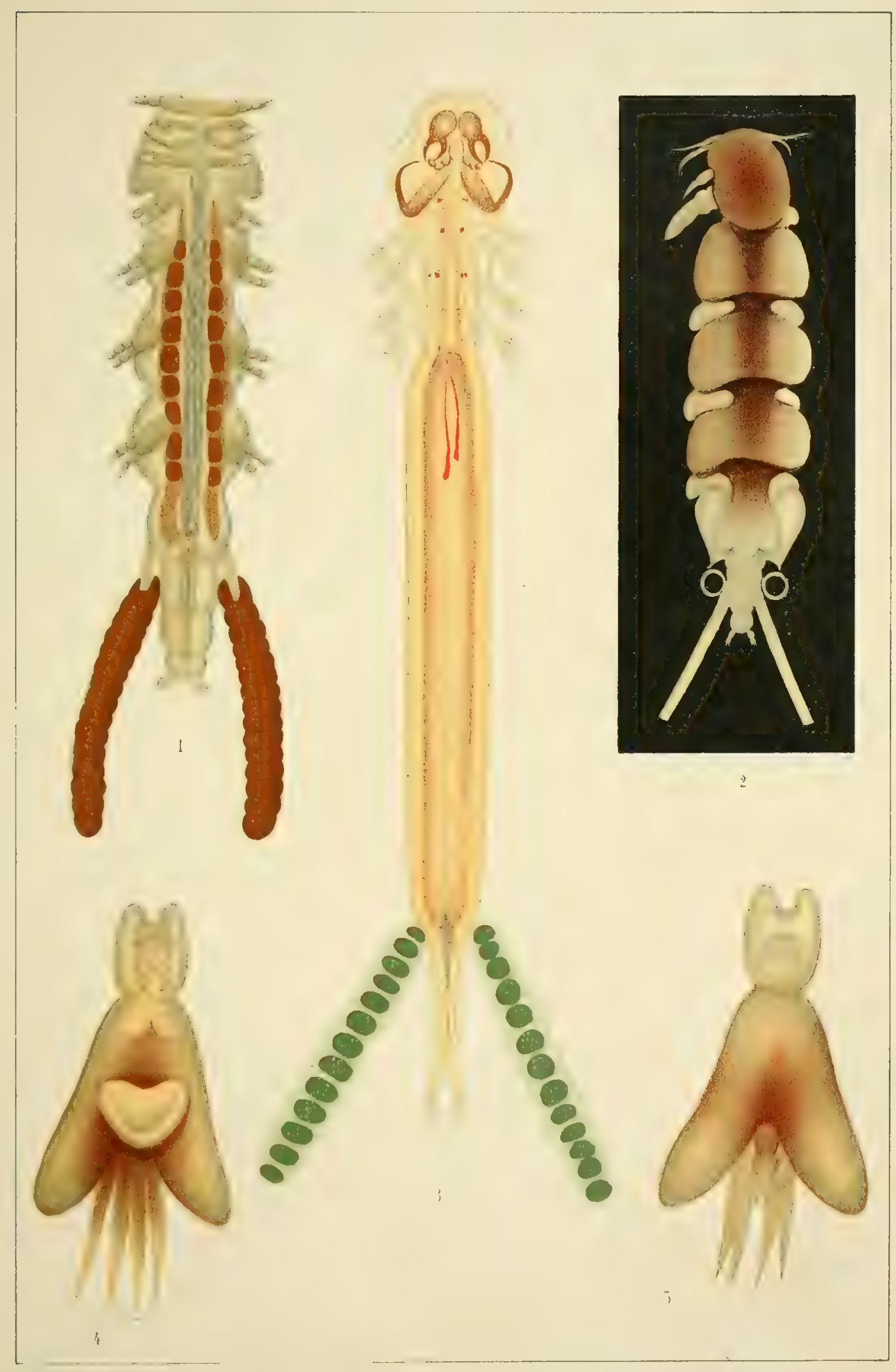

1. Eudactylina acuta. ㅇ.

2. Nemesis mediterravea, rar, sinirata $f$.

3. Krígeria lineata $q$.

4. To ermanthiopus trachuri, f. 



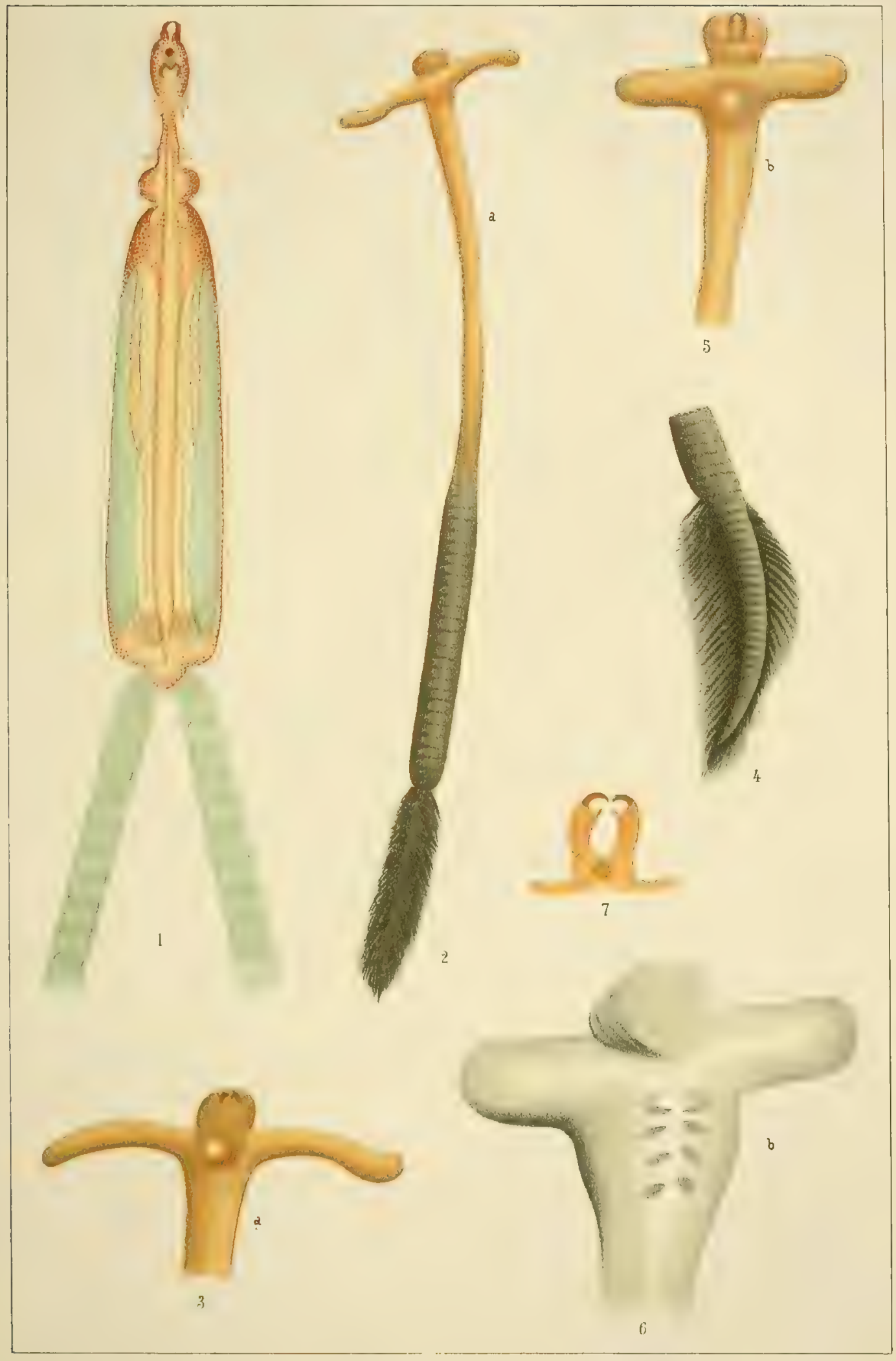

Lit Tacchinarai A Forani - Pavia.

1. Peniculus fistula. + .

2-7. Pennella crassicorncs. T. 


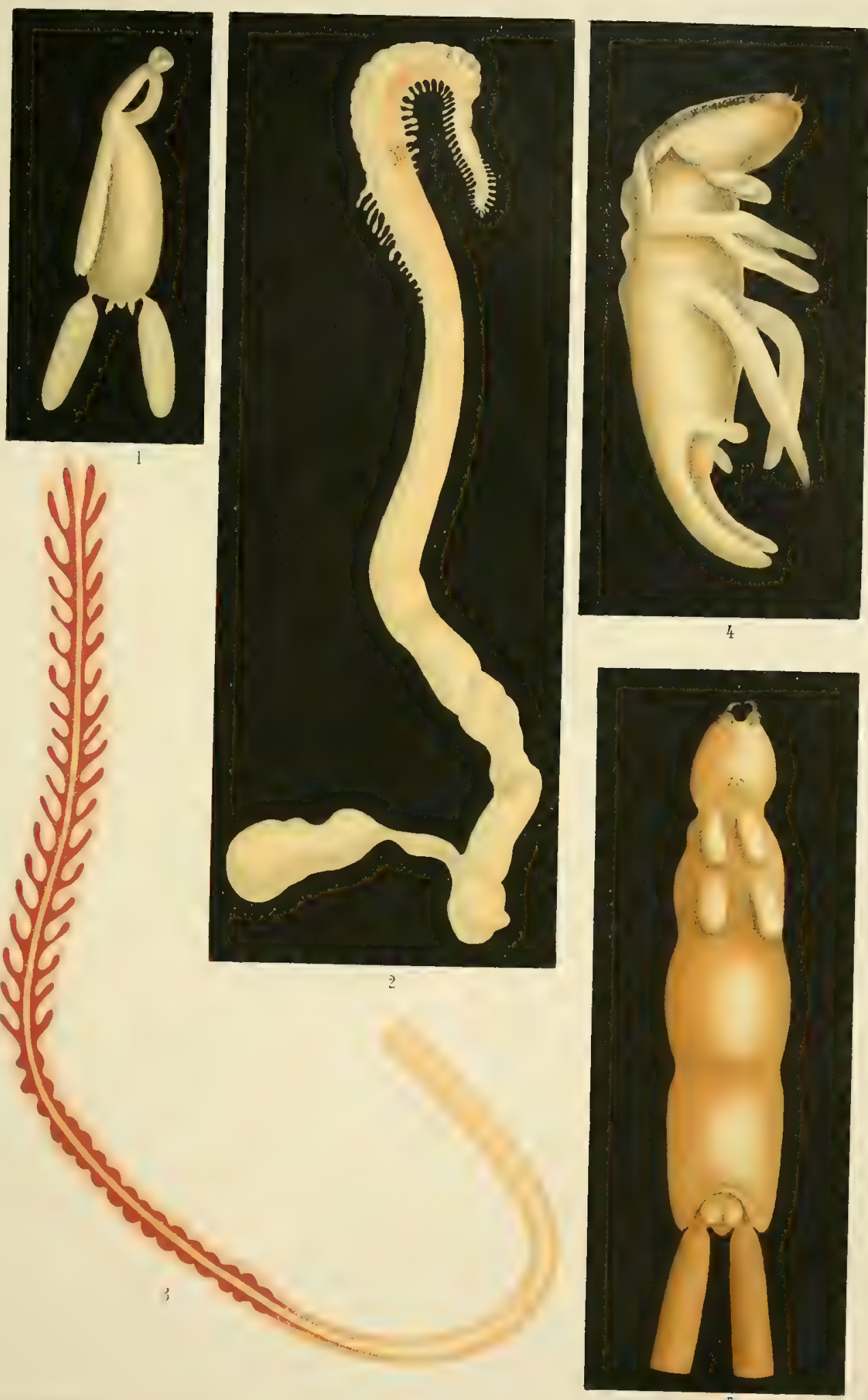

5

1. Brachiclla exigua. 7.

2. Peroderma bellottici.

3. Pennella ubra (2).

4. Choudracanthis merlucic ot.

5. Chondracanthus cormutis. + . 


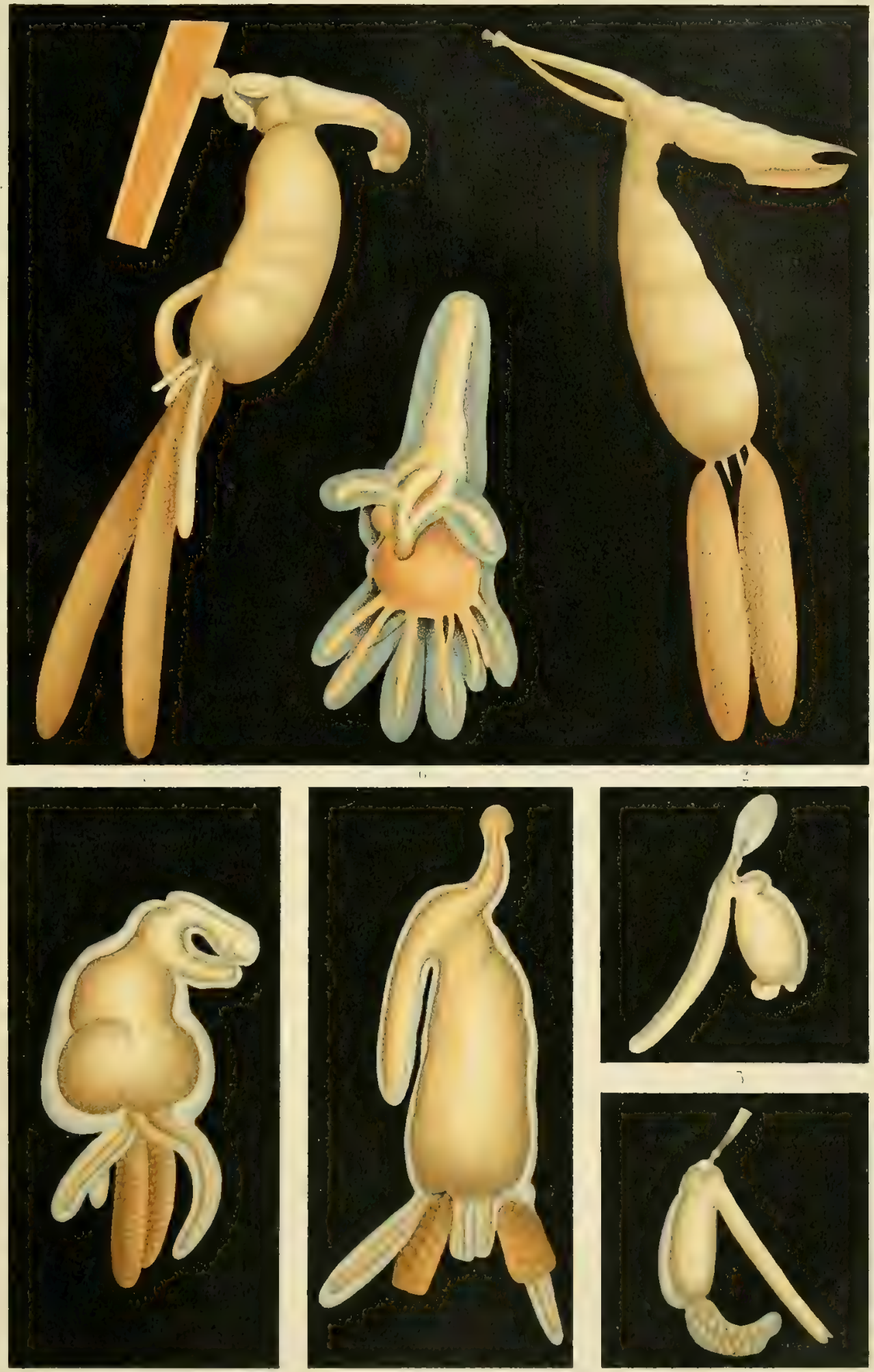

Brachiella insidiosa. ‥ 3.73nachiella merlucii. ㅇ. 2. Brachiella malleus.t. 5. Clavella macrotrachelus. 9 . 4. Brachiella insidiosa 6. Brachiella impudiea F. 



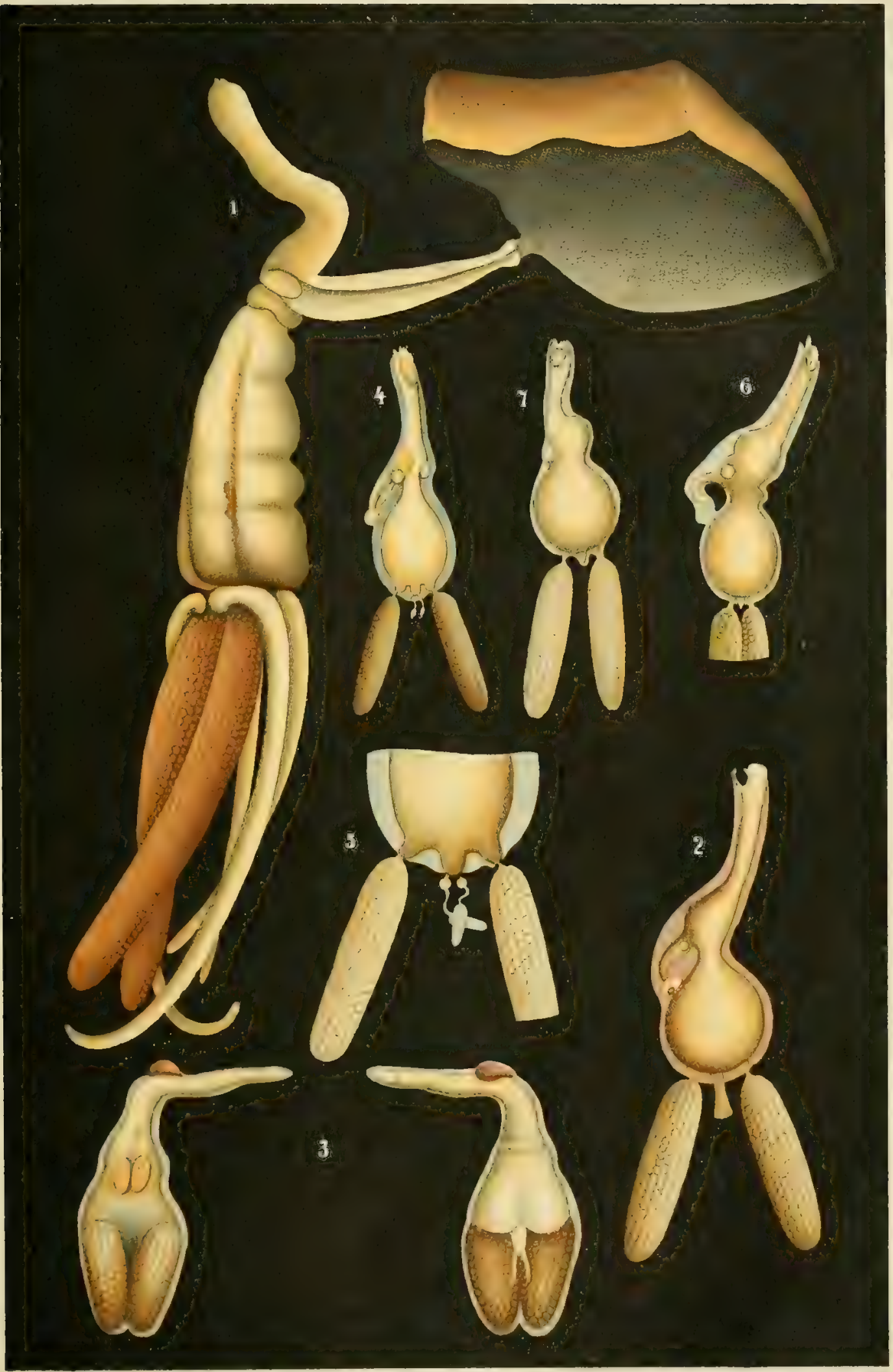

The

1. Brachiella thymi. ? 2. Clavella sangi, of. 3. Narbranchia cygnifformis + .
4. Claveilu siv.......... 우.

5. iden, abdonen - enlanged 6,7. idem, lateral. 


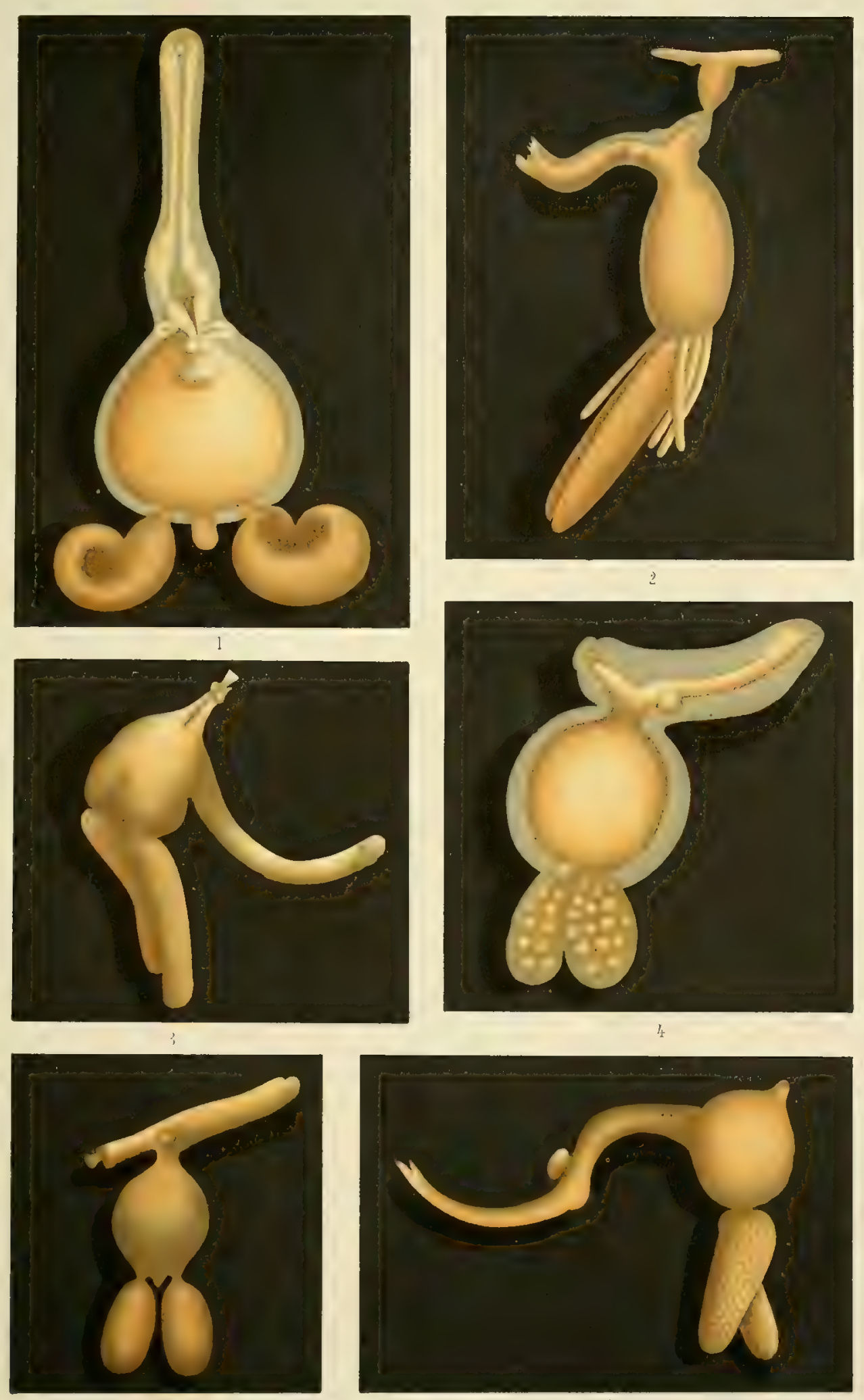

1. Clavella characis o 2. Clavella sciaenae.t. 3. Clavella emarginata.7. 4. Clavella fallax $q$. 5. Clavella pagri.7. 6. Clavella scambri ?. 

A.BRIAN-Copepodi parassili dei pesci d'talia

Tar.ty

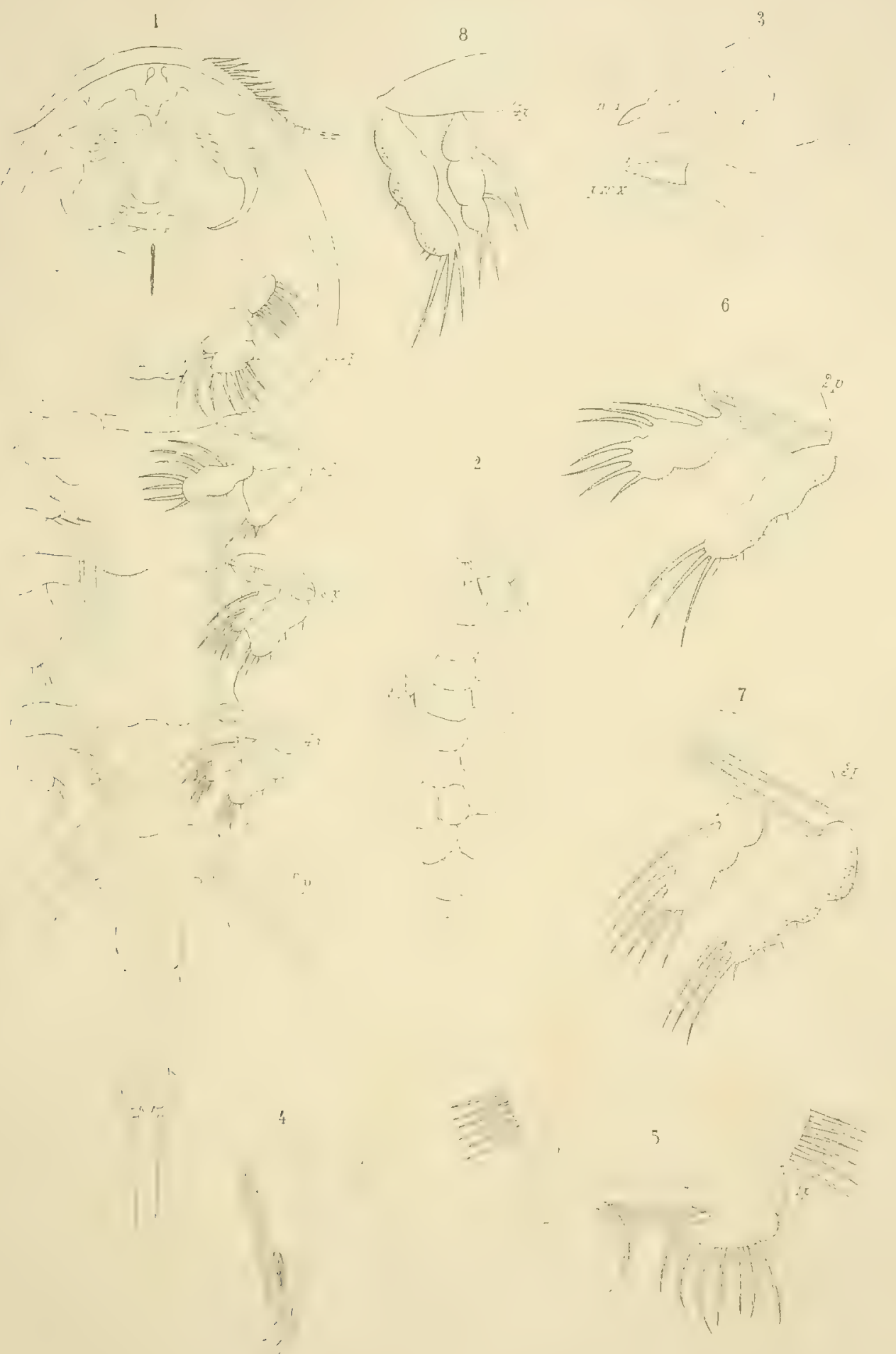

Psendocucanths alosse. 



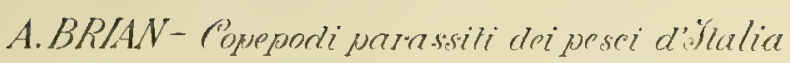

Tar: $|1|$

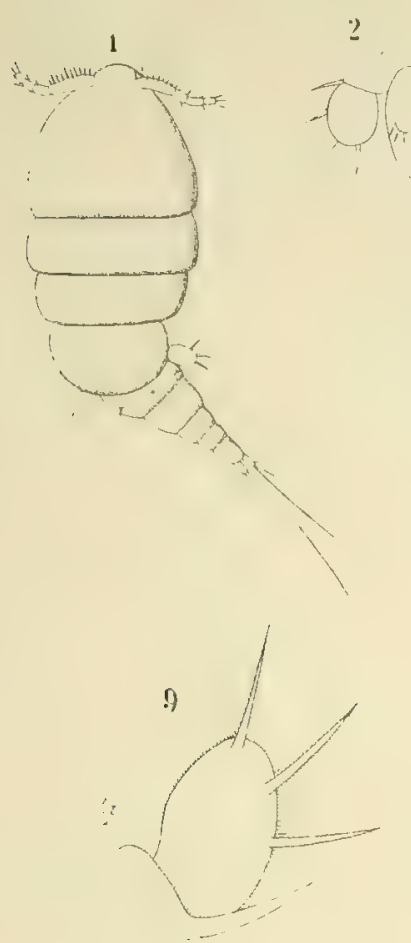

$\int_{i}(\cdots$

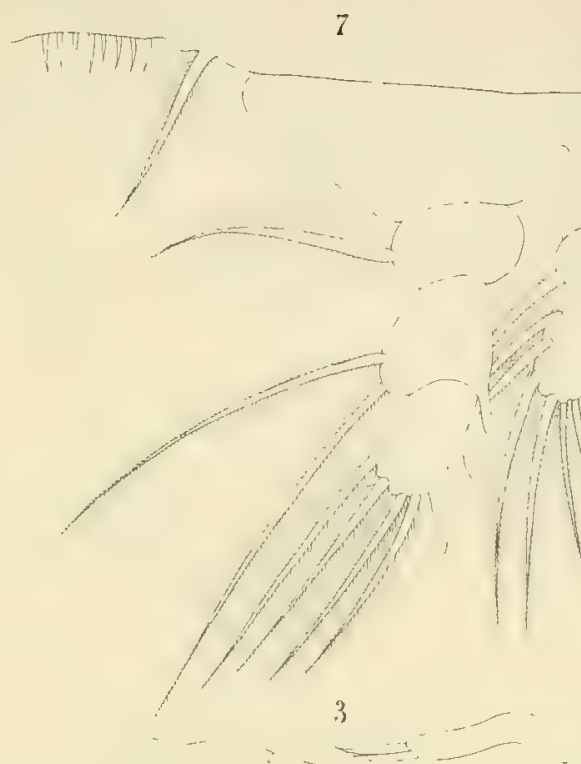

${ }^{4}, \quad \cdots / 1 / 1$

$\left.\right|_{1} ^{1}$

1

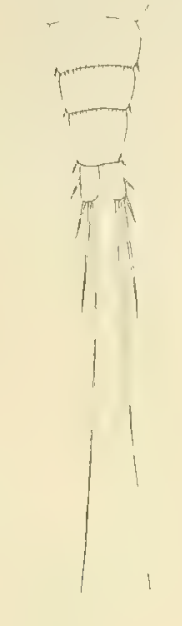

1

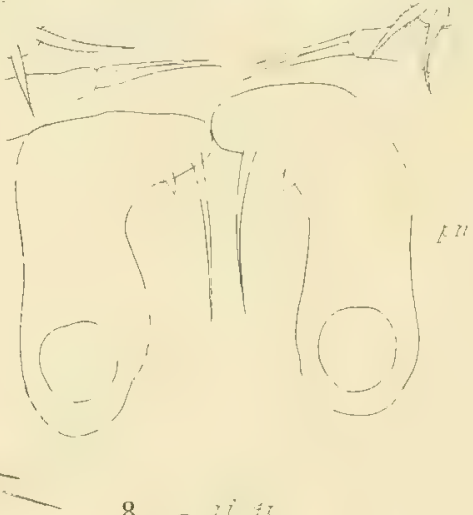

5

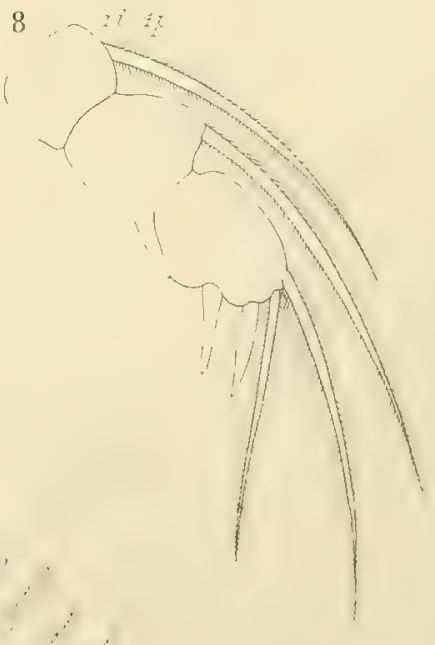

1. Tecokinardi o Frari- Favia.

Bomolochus (TRagus) muracuase. \& 

A. BRAN-Copeporti paressiti dei puesei whalia

7iv: $1 / 11$
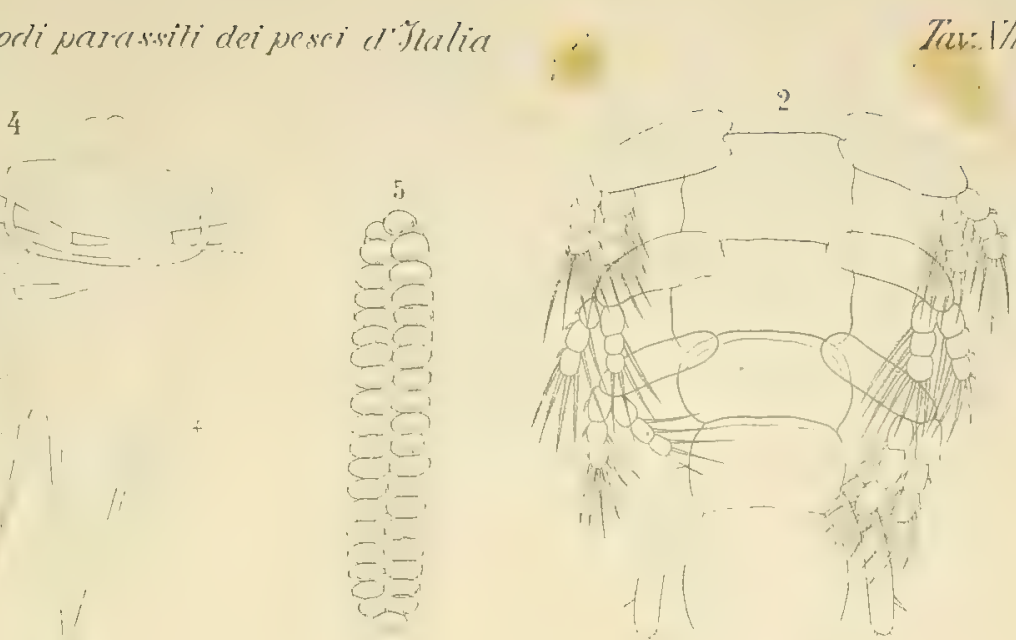

3

8

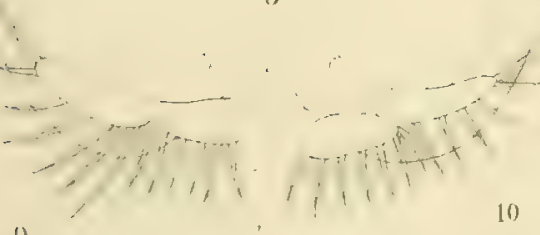

6
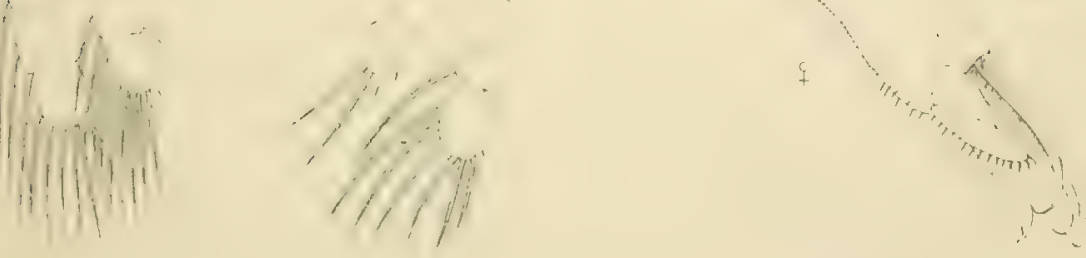

Anchistrotos gobii . ? and o (?). 

A. BRIAN-Copepodi parassiti dei peser d'Ylatia

Tar: $W^{\prime}$
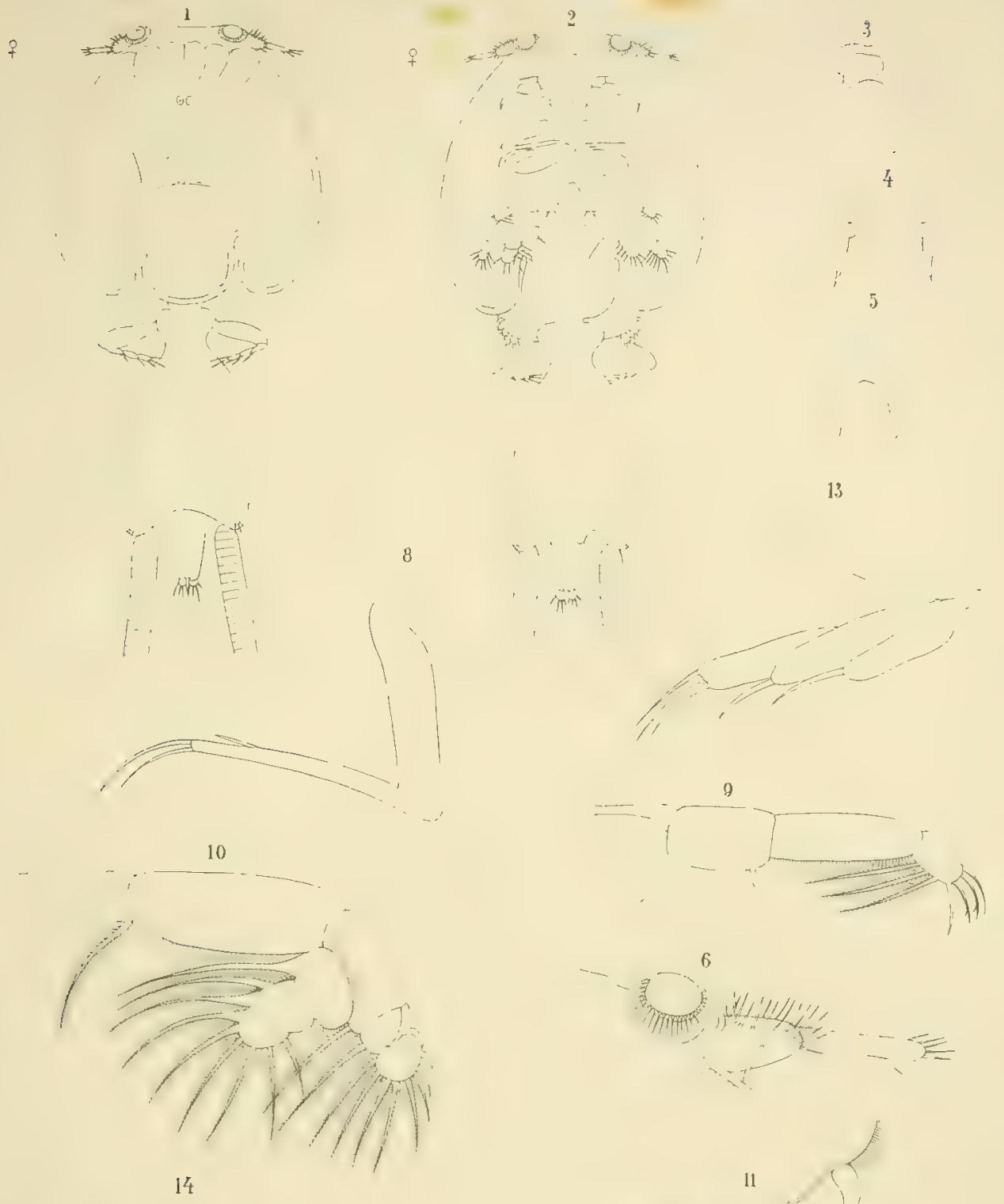

A

.

14

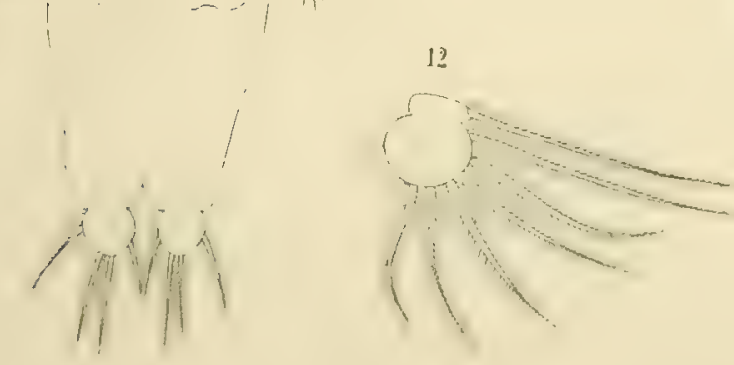

Lit Tacchinardi - Ferraini- Pavia

Caligus lichià of 



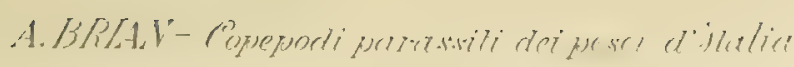

1. Tins 17

$-\bar{i}$

$\ldots$

8

Caligus ligusticus 3 . 

A. BRIAN-Copeporli parassiti dei pesei dTulia

Tin: 11

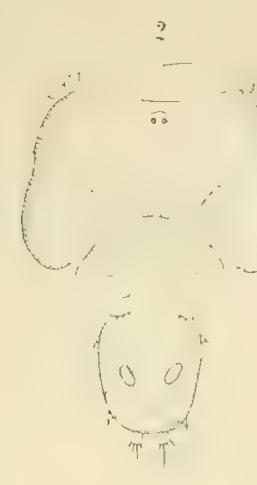

7

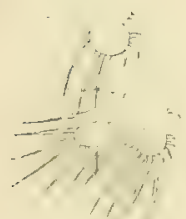

8

"

11

Philorttagoriscus serratus 3. figs. 1-7. Caligus lichiace ot figp. 8-11. 


pterygis Rich. (pag. 66); e scriva sotto la figura 2 Tav. XVII invece che il nome di L. Gisleri quello di L. Micropterygis.

Le ermanthropus trachuri of 7iq.1. Loernanthropus giokeri if 7 iq. 2 . Teernautteropus brevis of figo. 3-5. 

A.BRIAN-Copepodi parassiti dei pesci d'flatia

Tar: $1 \%$ III

8

12

if

Pennella filosa 9 figo. 1-Y.

Ternatenicus sp.? figo. 8-13. 

A. BRIAN-Copepodi parassiti dei pesci d'̌lalia

Tav.XIX.

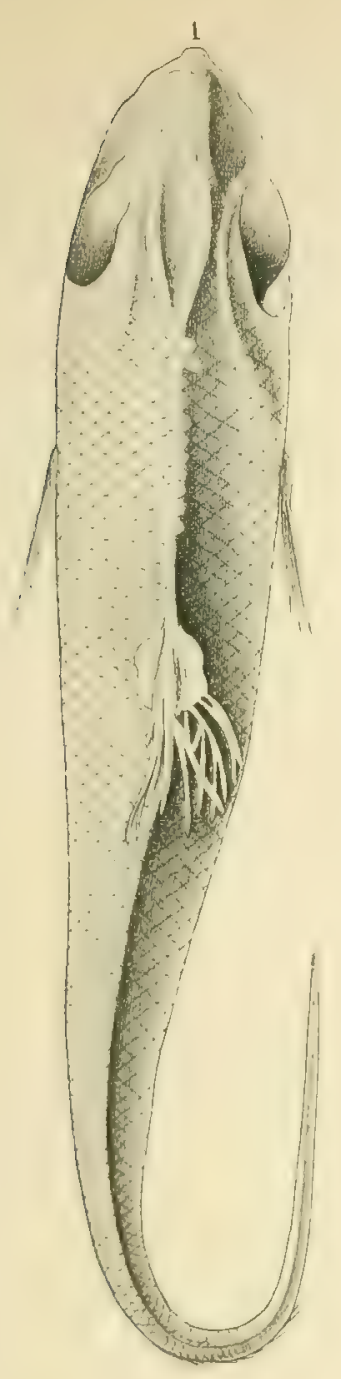

3

5

3

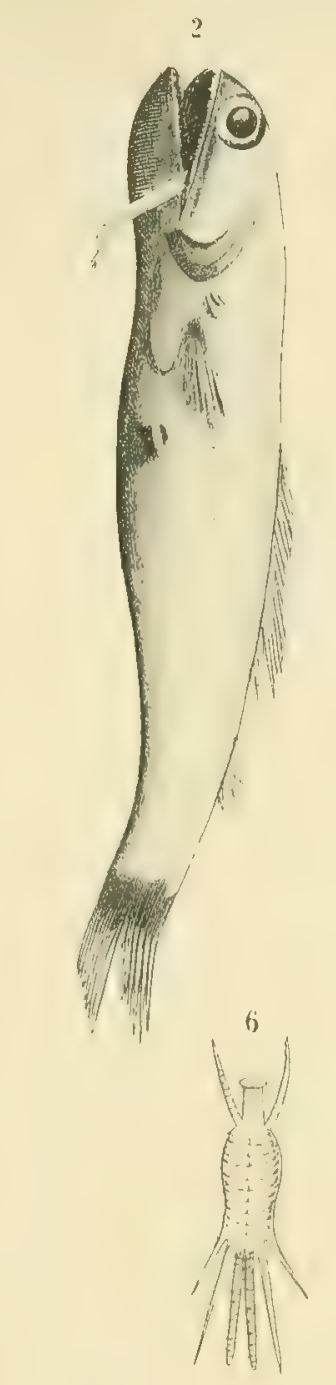

$-4 \pi$

Rebelula edurardsii of fig. 1 .

Peroderma bellattii of 7.is.2-6.5. 


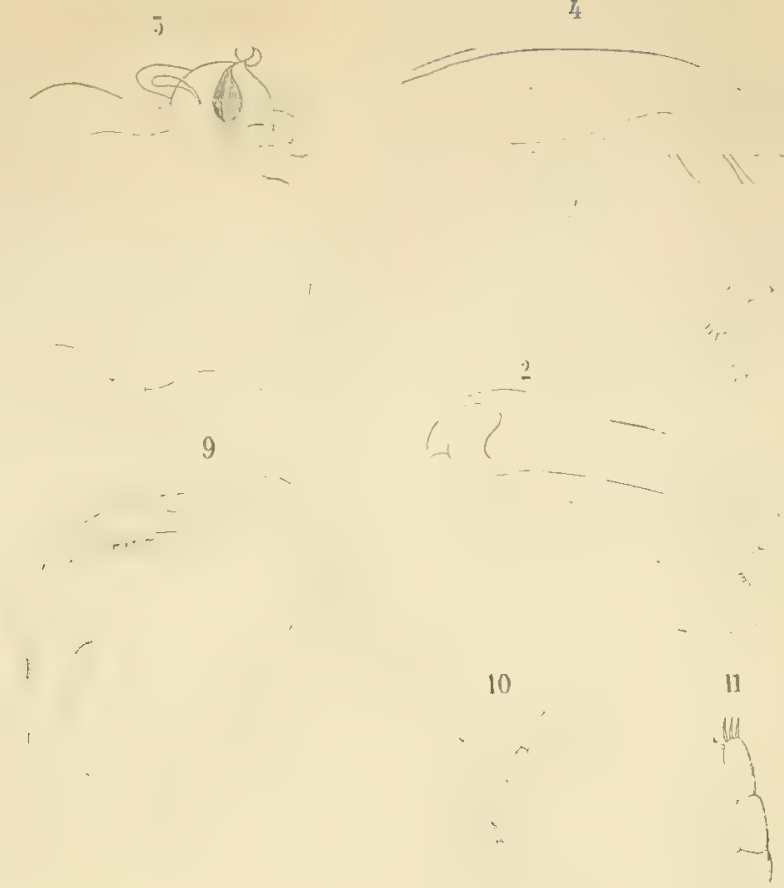

Hatscheleia sp. శ(?) jigo. 1-4.

Clavella alata of figo. 5-6. Brachiella exigua of fig. 7 . Clavella strumosa of Yigo.8-12. Clavella scinenae of fig - $13-14$. 


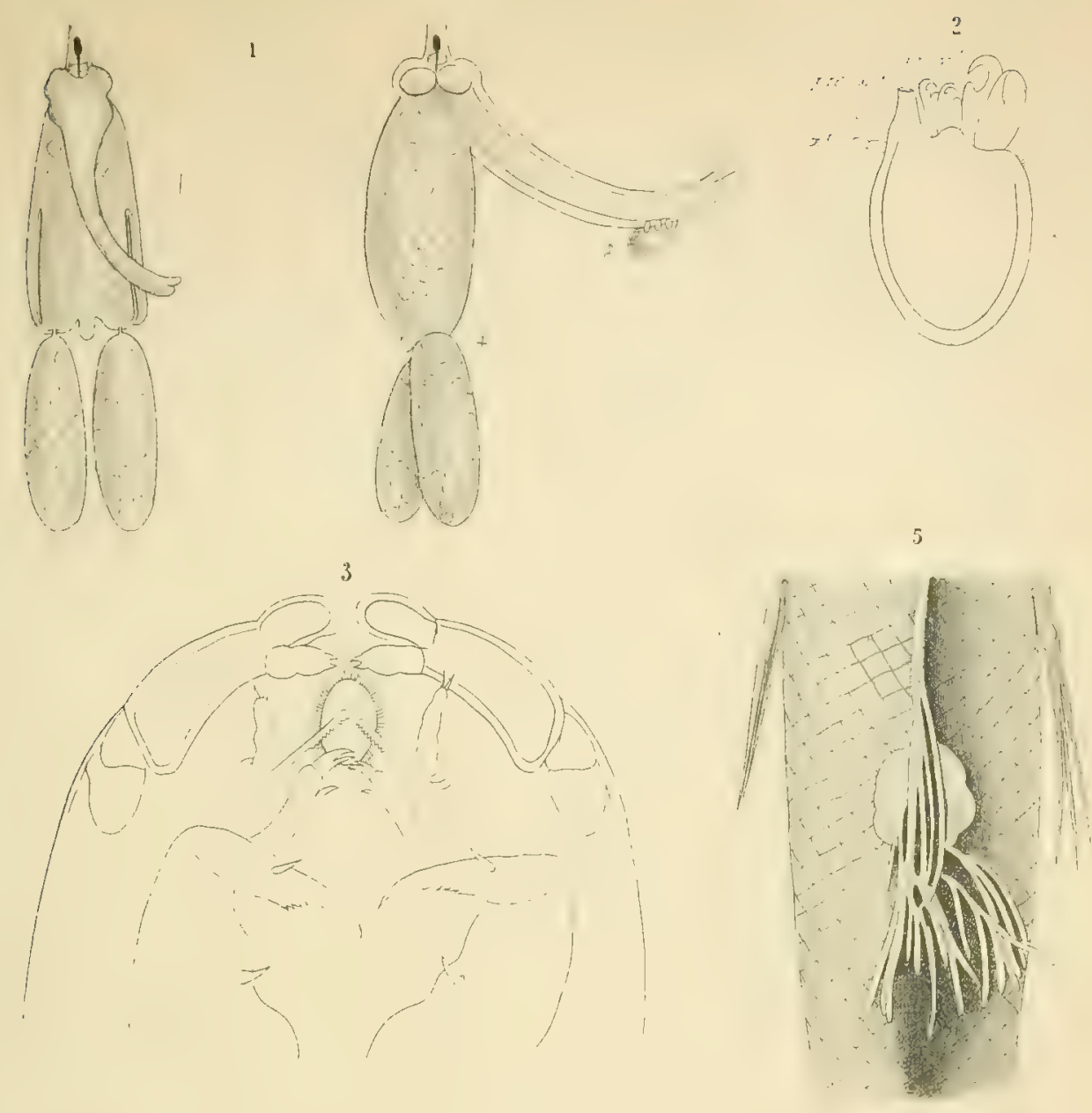

Clavella macerotrochelus of fig. 1-4.

Tebelula edwardsic of fig. 5 . Clavella dentiais of fig. 6 . 

WILSON COPEPOD LIBRARY

Smithsonian Institution

Invertebrate Zoology

(Crustacea) 

WILSON COPEPOD LIBRARY

Smithsonian Institution

Invertebrate Zoology

(Grustacea) - 

WILSON COPEPOD LIBRAPV

Smithsonian Instituliun Invertebrate Zoology

(Crustacea) 
